\title{
Grobwurzelarchitektur in Abhängigkeit von Baumart, Alter, Standort und sozialer Stellung
}

\author{
Dissertation
}

zur Erlangung des Doktorgrades der Fakultät für Forstwissenschaften und Waldökologie der Georg-August-Universität Göttingen

vorgelegt von Maike Kuhr geboren in Hildesheim 
1. Gutachter: Prof. Dr. von Lüpke

2. Gutachter: Prof. Dr. Gruber

Tag der mündlichen Prüfung: 21.01.2000 


\section{Vorwort}

Die vorliegenden Untersuchungen sind Teil eines gemeinsamen Projektes der Europäischen Union (AIR3-CT93-1269). Es wurden dabei verschiedene Fragestellungen zu "Funktionalen Aspekten der Wurzelarchitektur und der Biomasseverteilung von sechs europäischen Hauptbaumarten" bearbeitet. Das Institut für Waldbau der Universität Göttingen untersuchte hierbei Wurzeln der Fichte, Buche, Kiefer und Eiche. Die Daten wurden mit Hilfe einer gemeinsamen Datenbank verarbeitet und gingen in vergleichende Berechnungen mit Bäumen anderer Länder ein.

Dr. M. Drexhage wählte 1994 die Probeflächen aus und führte bis Mai 1996 erste Aufnahmen durch.

Mein herzlichster Dank gilt Prof. Dr. B. von Lüpke. Durch seine stete Bereitschaft zu Gesprächen und Gedankenaustausch sowie seine wertvollen Anregungen hat er ganz wesentlich zum Gelingen dieser Arbeit beigetragen.

Ganz besonders hervorheben möchte ich die kompetente und freundschaftliche Unterstützung durch Dr. S. Jaehne. Gemeinsame Diskussionen, die inhaltliche, statistische und formale Probleme bei einem Glas Wein lösten, waren bei der Erstellung der Arbeit von unschätzbarem Wert.

Ebenfalls durch ihr besonderes Engagement entlasteten mich technische, studentische und wissenschaftliche Mitarbeiter, namentlich K.- H. Obal, P. Heinze, U. Westphal, A. Nannen, M. Unger, A. Parth, A. Horn, T. Kappes, A. Heidenfelder, K. DeWall, H. Dzierzon, H. Beuke, Dr. U. Sittig, Dr. N. Bartsch, Dr. M. Drexhage, Dr. D. Murach und I. Bechtold. Für die Lösung verwaltungstechnischer Probleme waren I. Albrecht und K. Gruel unerläßlich. Dafür möchte ich mich an dieser Stelle bei allen genannten Personen ganz herzlich bedanken.

Nicht zuletzt sei die freundliche Unterstützung durch die Forstämter Kattenbühl, Knesebeck, Holzminden und Bovenden hervorzuheben.

Göttingen im Januar 2000

Maike Kuhr 


\section{INHALTSVERZEICHNIS}

\section{EINLEITUNG}

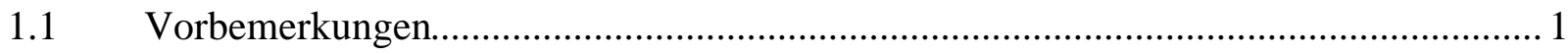

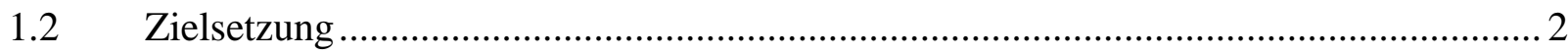

Einführung in den Aufbau der Arbeit .............................................................. 3

\section{MATERIAL}

2.1 Standörtliche Beschreibung der Versuchsflächen

2.1.1 Beschreibung der geographischen Lage und des Klimas ................................................ 4

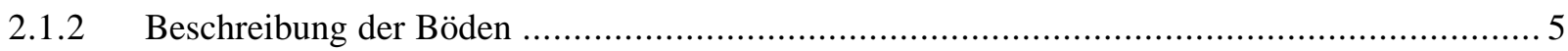

2.2 Untersuchungsbestände

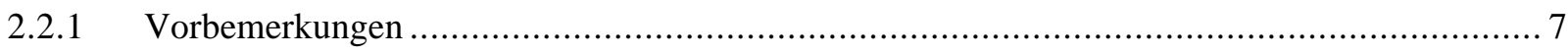

2.2.2 Untersuchungsgebiet Bovenden (ehemals Radolfshausen), Fichte und Buche

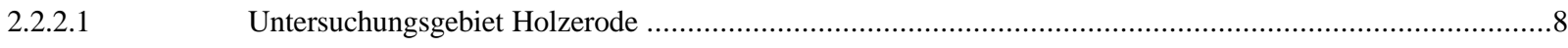

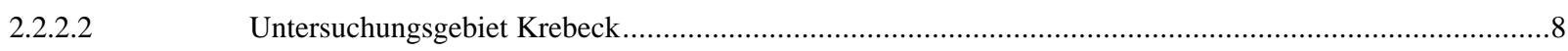

2.2.3 Untersuchungsgebiet Knesebeck, Kiefer und Fichte ....................................................... 10

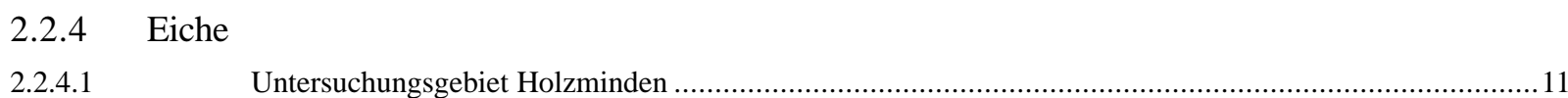

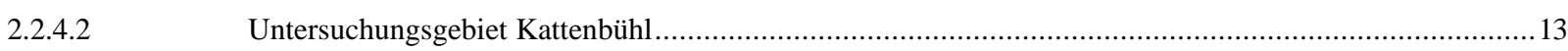

\section{METHODEN}

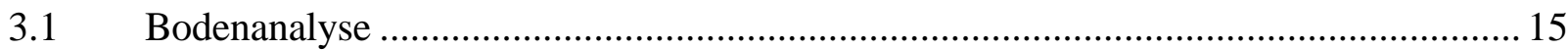

3.2 Auswahl und Erfassung der Probebäume ........................................................... 15

3.3 Konkurrenzbedingungen

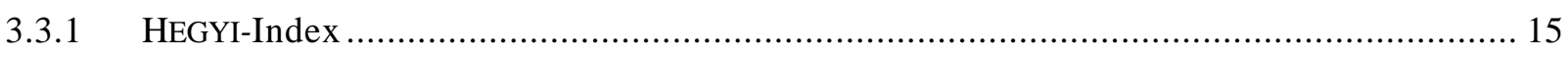

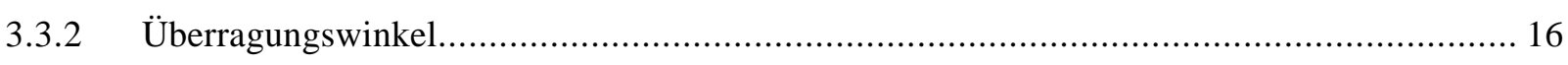

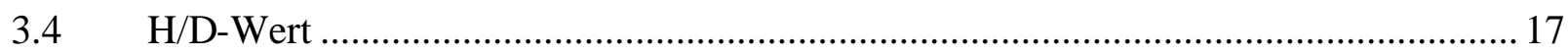

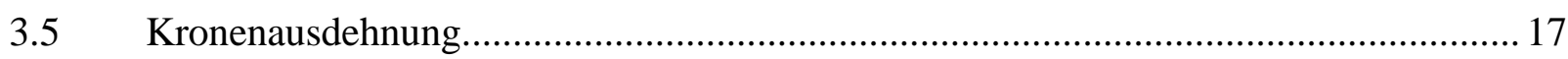

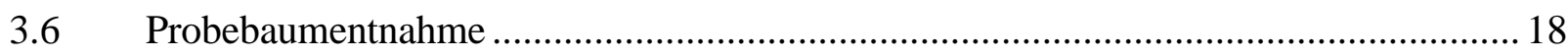

3.7 Gewinnung und Aufnahmeverfahren der ober- und unteridischen Biomasse .............. 18

3.8 Ermittlung der Grobwurzel- und Feinwurzelparameter

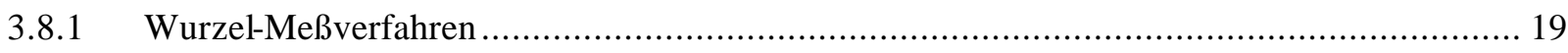

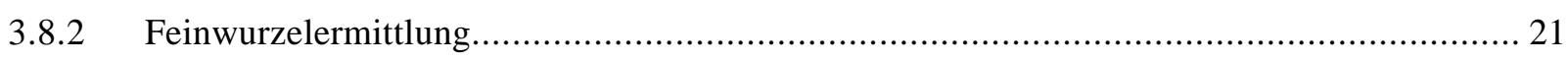

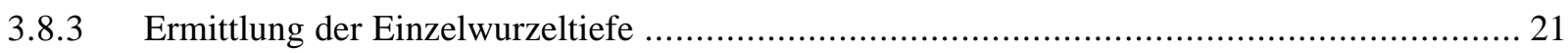

3.9 Zusammenfassung der erhobenen Parameter

3.10 Datenanalyse und Statistik

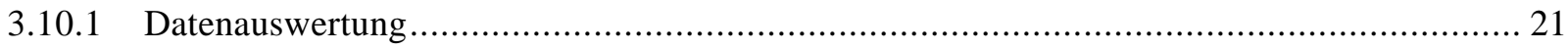

3.10.2 Weiterführende Berechnungen der Wurzelparameter................................................ 22

3.10.3 Erstellung der Häufigkeitsverteilungen der Wurzeln der ersten Sektion 
3.11 Erstellen von Flußdiagrammen zur Beschreibung der oberirdischen- und unterirdischen Parameter.

\section{ERGEBNISSE}

\subsection{Oberirdische Parameter}

4.1.1 Konkurrenzbedingungen

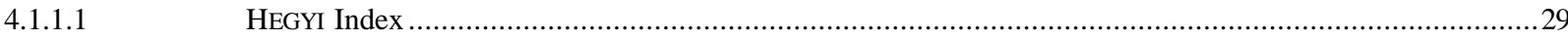

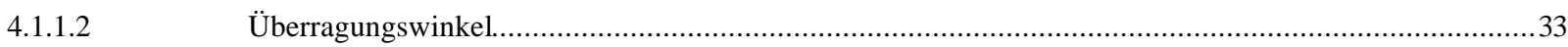

4.1.2 Probebaumbeschreibende Parameter (BHD, Höhe, H/D-Wert, Stammvolumen)

Anzahl der Probebäume und soziale Stellung ........................................ 34

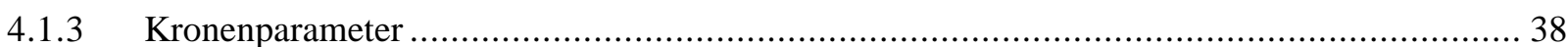

\subsection{Wurzelparameter}

\subsubsection{Einzelwurzel}

4.2.1.1 Wurzeldurchmesserverteilung

4.2.2 Wurzelsystem

Tiefendurchwurzelung

Verteilung der Wurzelanzahl und Wurzelquerschnittfläche auf die drei horizontalen und vertikalen Sektionen am Beispiel der Eiche; Holzminden

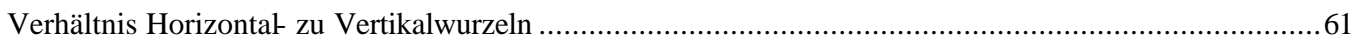

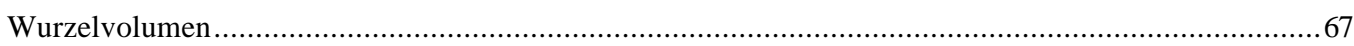

Wurzelvolumen differenziert nach sozialer Stellung................................................................... 70

Wurzelvolumen der Probebäume in einem definierten Bodenraum ..................................................... 71

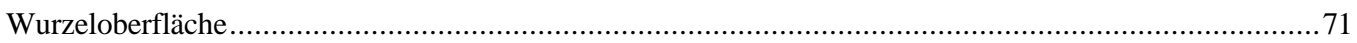

\subsection{Sproß / Wurzelbeziehungen}

4.3.1 Beziehungen zwischen dem "HEGYI-Index" und dem Wurzelvolumen

4.3.2 Beziehungen zwischen ober- und unterirdischen Parametern 


\section{DISKUSSION}

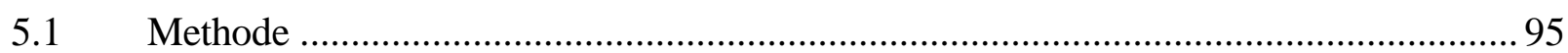

5.2 Veränderung der Wurzelarchitektur bei Mischung von Buche und Fichte

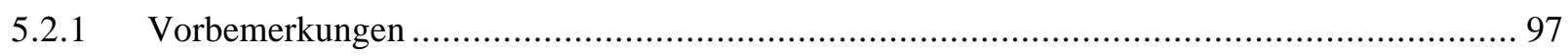

5.2.2 Tiefendurchwurzelung .................................................................................... 98

5.2.3 Wurzelintensität

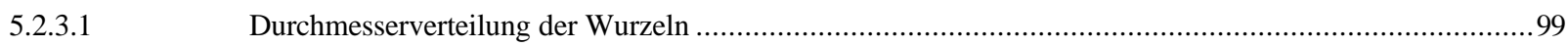

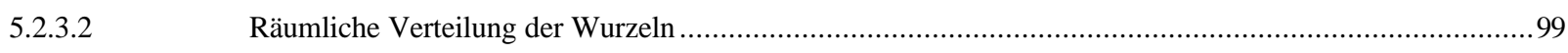

5.2.4 Sproß / Wurzelbeziehungen........................................................................................101

5.3 Kiefer und Fichte in 20,30 und 50 jährigen Reinbeständen

5.3.1 Wurzelentwicklung der Kiefer auf Sand

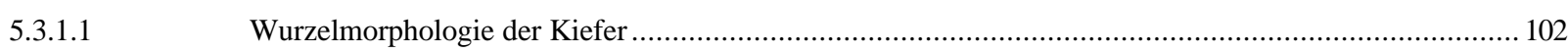

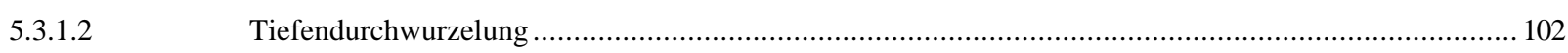

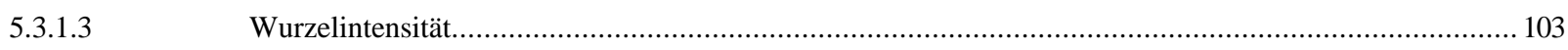

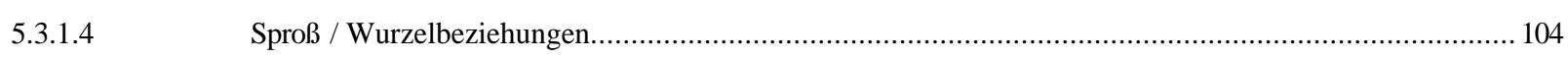

5.3.2 Die Wurzelentwicklung der Fichte auf Sand

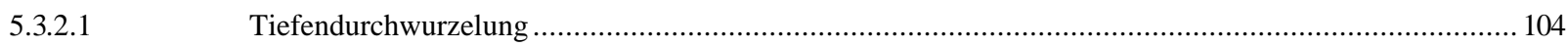

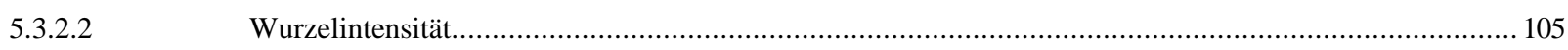

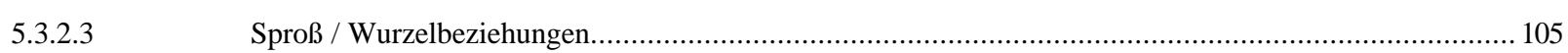

5.3.3 Wurzelentwicklung der Fichte auf Lehm ...........................................................

5.4 Einfluß des Bodens auf das Wurzelwachstum 30 jähriger Fichten (Sand / Lehm)

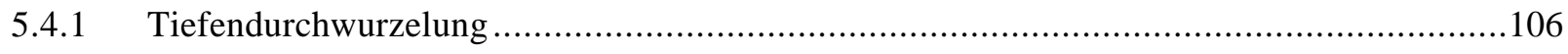

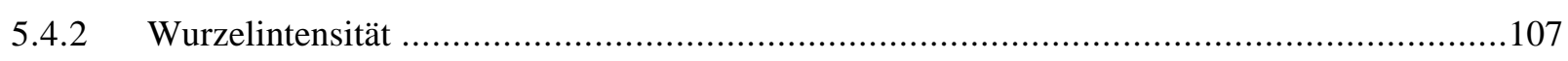

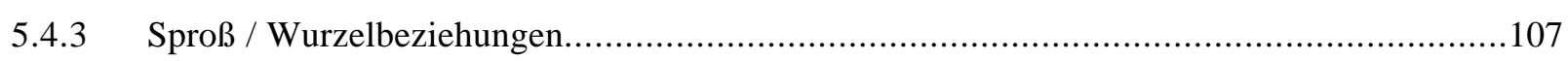

5.5 Einfluß der Verjüngungsmethode und unterschiedlicher Bodentypen (Stagnogley, Lehm) auf die Wurzelausbildung der Eiche

5.5.1 Eiche auf problematischen Böden (Stagnogley) .................................................

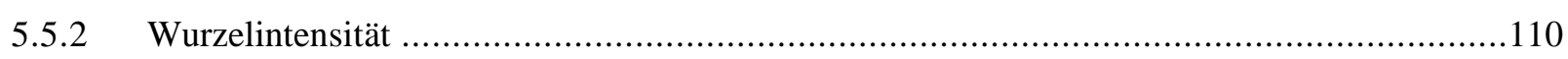

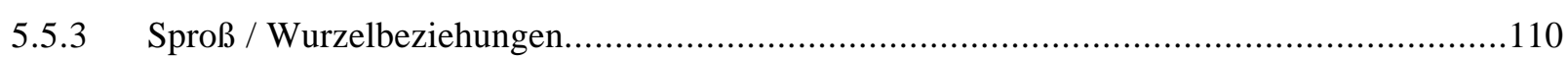

5.6 Einfluß der sozialen Stellung auf die ober- und unterirdischen

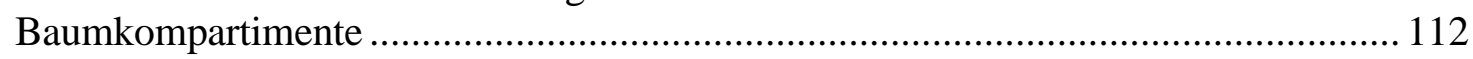

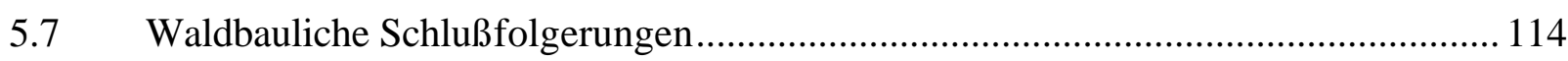

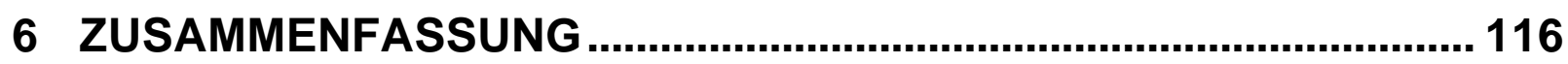

7 GLOSSAR ................................................................................... 120

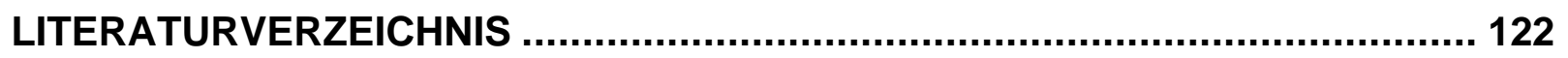

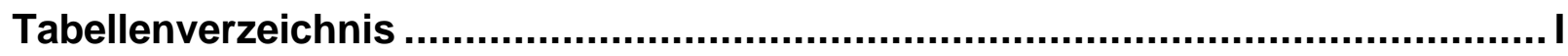

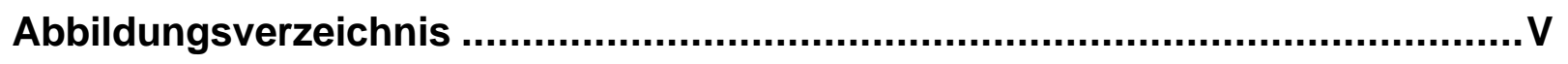

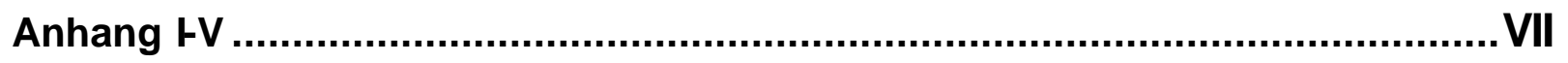




\section{Einleitung}

\subsection{Vorbemerkungen}

Da Grobwurzeluntersuchungen besonders aufwendig sind, gibt es über die Wurzeln von Waldbäumen weit weniger Untersuchungen als über oberirdische Organe. Bis Ende der 60er Jahre werden lediglich allgemeine, wenig exakte Beschreibungen durchgeführt. Datenmaterial einzelner Wurzelsysteme ist schon zu Beginn dieses Jahrhunderts von LiESE (1926), HILF (1927), VATER (1927), BRÜCKNER und JAHN (1932) und KRAUSS (1938) erstellt worden. Diese Ergebnisse erklären die Wurzelausprägungen verschiedener Baumarten jedoch nicht im ausreichenden Maße, da die Stichprobengröße sehr gering ist. Die gefundenen Resultate standen in keinem Verhältnis zu dem hohen Aufwand an Zeit und finanziellen Mitteln.

Karl GAYER stellte schon 1867 die Forderung nach "Vergleichenden Versuchen über die Wurzelbildung der verschiedenen Holzarten auf verschiedenen Böden". Dieses Ansinnen griffen KÖSTLER et al (1968) auf und vergleichen in ihrem Werk "Die Wurzeln der Waldbäume" eigene Erkenntnisse mit denen der Literatur, die sich ebenfalls zum größten Teil auf Ausgrabungen einzelner Wurzelsysteme beziehen.

In den 80er Jahren erhöht sich das Interesse an Wurzeln im Hinblick auf die Biomasse Produktivität von Waldökosystemen, und man stellt fest, daß über den Anteil der Wurzeln an der Biomasse nur sehr wenig Konkretes bekannt ist. Z.B. reichen die Schätzungen über den Anteil der Feinwurzelumsetzung an der Nettoprimärproduktion von $8 \%$ (KEYES und GRIER 1981) bis $67 \%$ (GRIER et al 1981). Übersichten entsprechender Untersuchungen in verschiedenen Waldbeständen sind bei FOGEL (1985) und SANTANTONIO (1989) zu finden.

Ein nächster Anstoß zur Erhebung von Wurzeldaten entwickelt sich aus der Fragestellung der sogenannten "neuartigen Waldschäden", da man annimmt, daß durch die Bodenversauerung Wurzelschädigungen auftreten. (ULRICH et al 1984, RAPP und JENSCHKE 1994).

Veranlaßt durch schwere Windwürfe Anfang der 90er Jahre, sind in den letzten Jahren genauere Untersuchungen von Grobwurzelsystemen durchgeführt worden (EICHHORN 1992, ZOTH und BLOCK 1992, ALDINGER et al 1996, MÖßMER 1997).

Die Baumarten werden in Pfahl-, Flach- und Herzwurzler gegliedert (BÜSGEN und MÜNCH 1927). Viele weiterführende Untersuchungen machen deutlich, daß diese Klassifikation innerhalb derselben Baumart großen Variationen unterliegen kann. So kann beispielsweise die als Flachwurzler bekannte Fichte Tiefenwurzeln ausbilden (KALININ 1978) und die als Herzwurzler bezeichnete Buche Flachwurzeln (POLOMSKI und KUHN 1998).

Grobwurzeln (Wurzeln mit einem Durchmesser $>5 \mathrm{~mm}$ ) haben in erster Linie die Funktion, den Baum im Boden zu verankern (STOKES und GUITARD 1997; ATGER und EDELIN 1993). Daneben sind sie Träger der feineren Wurzeln (Durchmesser $<5 \mathrm{~mm}$ ), die vorwiegend der Wasser- und Nährstoffaufnahme des Baumes dienen (RAVEN et al 1988). Bei manchen Baumarten tragen die Wurzeln zudem durch Wurzelbrut zur vegetativen Vermehrung bei. Die Aufgliederung des Wurzelsystems erfolgt nach einem Verzweigungsschema, das, differenziert nach Horizontal- und Vertikalebenen, als Architektur bezeichnet wird. Abhängig ist diese Architektur von der Baumart und der Anpassung an die ökologischen Bedingungen (GRUBER 
1992), wobei zunächst die physikalischen und chemischen Bodenverhältnisse (NIELSEN 1990) einen großen Einfluß haben. Weiterhin spielt die Kronenmasse eine entscheidende Rolle (MELZER 1962).

Neben den abiotischen Faktoren, Bodengefüge, Nährstoff- und Wasserversorgung, Exposition, Licht und Temperatur, beeinflussen vermutlich folgende Größen das Wurzelwachstum. Dies sind vor allem die Art der Entstehung (Naturverjüngung, Saat, Pflanzung oder vegetative Vermehrung) sowie die gesteuerte oder nicht gesteuerte Weiterentwicklung des Bestandes. Hieraus entstehen inter- und intraspezifische Konkurrenzbedingungen, die sich aus unterschiedlichen waldbaulichen Behandlungen ergeben (NIELSEN 1990). Diese Konkurrenzbedingungen werden sichtbar durch die Dichte und Struktur der Bestände.

Bekanntermaßen wirken sich diese Faktoren auf die Krone aus, die sicherlich zum großen Teil die Wuchsbedingungen der Bäume steuert (KOZlOwSKI und PALlARDY 1997; GRACE 1997). Oberirdische Parameter wie Höhen-, Durchmesser- und Kronenentwicklung sind ausführlich sowohl in der Waldwachstumskunde als auch im Waldbau untersucht worden. Unbekannt ist dagegen deren Zusammenhang mit der Wurzelausbildung. Ebenfalls nicht geklärt ist, inwiefern umgekehrt die Wurzeln das Wachstum oberirdischer Größen beeinflussen können. Dieses soll in der vorliegenden Arbeit in Zusammenhang mit ökologische Faktoren untersucht werden.

\subsection{Zielsetzung}

Oben gesagtes läßt erkennen, daß hinsichtlich der Grobwurzeln nur wenige Forschungsergebnisse verfügbar sind. Ziel dieser Arbeit ist es, eine Verteilung horizontaler und vertikaler Wurzelparameter herauszustellen. Dazu werden die Grobwurzeln und oberirdische Parameter der Baumarten Fichte, Kiefer, Eiche und Buche auf unterschiedlichen Standorten untersucht. Die Architektur der Wurzelsysteme wird beschrieben durch ihre Ausdehnung in die Tiefe und in die Horizontale, durch die Wurzelanzahl und -masse.

In einem ersten Schwerpunkt wird geprüft, ob und inwiefern sich der Aufbau der Grobwurzelarchitektur bei Mischung der Baumarten Fichte und Buche verändert. Anlaß zu diesen Untersuchungen geben Arbeiten von MACKENTHUN (1990) und FÖLSTER et al (1991), die berichten, daß bei Mischung von Fichte mit Buche eine Verflachung der Fichtenfeinwurzeln und eine Tiefenverlagerung der Buchenfeinwurzeln stattfinden. Es soll geprüft werden, ob dies für die Grobwurzelverteilung auch zutrifft. Weiterhin wird an diesen Flächen die Verschiedenheit der Wurzelausbildung von rund 60 jährigen Buchen und Fichten im Reinbestand untersucht und mit denen des Mischbestandes verglichen.

Einen weiteren Schwerpunkt bilden Untersuchungen in 20-, 30- und 40- bis 50 jährigen Fichten-und Kiefern-Reinbeständen. Die Bestände jeder Baumart stocken auf gleichen Standorten und erlauben daher eine Einschätzung der Entwicklung von Wurzelsystemen und oberirdischen Größen in Abhängigkeit vom Alter. Zusätzlich wird an 30 jährigen Fichtenbeständen auf Sandpodsol und schwach pseudovergleyter Parabraunerde der Einfluß unterschiedlicher Bodenverhältnisse auf die Wurzelentwicklung dargestellt. 
An der Eiche soll bearbeitet werden, wie sich verschiedene Begründungsverfahren, Pflanzung und Saat, auf die Entwicklung des Grobwurzelsystems auswirken. Dies wird auf zwei Standorten untersucht.

Bei allen Baumarten wurden Probanden aus verschiedenen sozialen Stellungen gewählt. An diesen Bäumen wird erörtert, wie sich mit zunehmender Konkurrenz die ober- und unterirdischen Parameter verändern.

Im folgenden wird die Zielsetzung in Kurzform dargestellt.

- Welchen Einfluß hat die Mischung der Baumarten Fichte und Buche auf die Entwicklung des Wurzelsystems?

- Welchen Einfluß hat Konkurrenz anderer Bäume (soziale Stellung) auf die Wurzelarchitektur verschiedener Baumarten?

- Wie entwickelt sich die Wurzelarchitektur 20-, 30- und 50 jähriger Fichte und Kiefer auf gleichem Standort?

- Vergleich der Wurzelarchitektur von 30 jährigen Fichten auf verschiedenen Standorten.

- Wie unterscheidet sich die Wurzelarchitektur gesäter von der gepflanzter Eichen?

\subsection{Einführung in den Aufbau der Arbeit}

Gegliedert sind die Ergebnisse in drei Abschnitte. Im ersten werden die oberirdischen Parameter und in einem zweiten die Wurzelparameter dargestellt. Der dritte Abschnitt setzt die oberund unterirdischen Baumparameter ins Verhältnis.

Zu den oberirdischen Faktoren zählen Konkurrenzbedingungen, ertragskundliche Daten und Kronenparameter (Probebäume). Dabei gehören in dieser Arbeit Alter, BHD [cm], Höhe [m], Stammvolumen $\left[\mathrm{m}^{3}\right], \mathrm{H} / \mathrm{D}-$ Wert und soziale Stellung zu den ertragskundlichen Daten. Zu den Kronengrößen werden Kronengewicht (trocken) [kg], welches getrennt nach Blattmasse, Ast-

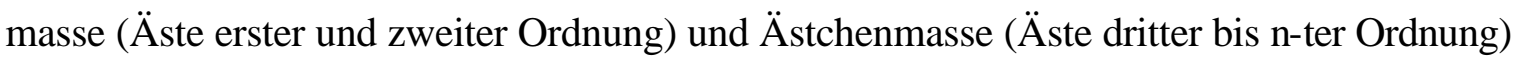
dargestellt ist, Kronenansatz, Kronenlänge und Kronenprozent gezählt. In zwei Beständen Kronenablotungen zur Ermittlung der Kronenschirmfläche vorgenommen. werden

\section{Wurzelparameter}

Es wird unterschieden zwischen Einzelwurzel und Gesamtwurzelsystem eines Baumes. Ersterer Begriff ist in dem Abschnitt Einzelwurzelmorphologie zu finden, letzterer im Abschnitt Wurzelsysteme.

\section{Beziehungen zwischen ober- und unterirdischen Parametern}

Hierzu werden Regressionen zwischen Konkurrenz-, Stamm- und Kronenparametern im Verhältnis zu Wurzelgrößen aufgestellt.

\section{Aufbau der Ergebnisdarstellung}

Grundsätzlich werden die Ergebnisse in folgender Reihenfolge dargestellt:

- Fichte und Buche im Rein- und Mischbestand (Untersuchungsbestand Holzerode)

- Fichte und Kiefer 20- bis 50 jährig (Untersuchungsbestände Krebeck und Knesebeck)

- Eiche im Vergleich Saat und Pflanzung (Untersuchungsbestände Holzminden und Kattenbühl 


\section{Material}

\subsection{Standörtliche Beschreibung der Versuchsflächen}

\subsubsection{Beschreibung der geographischen Lage und des Klimas}

Die Untersuchungen werden an den Baumarten Fichte (Picea abies (L.) Karst), Eiche (Quercus robur ssp. petraea Kl. et Kr. et Rol.), Kiefer (Pinus sylvestris L.) und Buche (Fagus sylvatica L.) durchgeführt. Das Untersuchungsmaterial stammt aus Beständen der niedersächsischen Forstämter Bovenden (ehemals Radolfshausen), Knesebeck (ehemals Sprakensehl), Holzminden und Kattenbühl (Abb. 1).

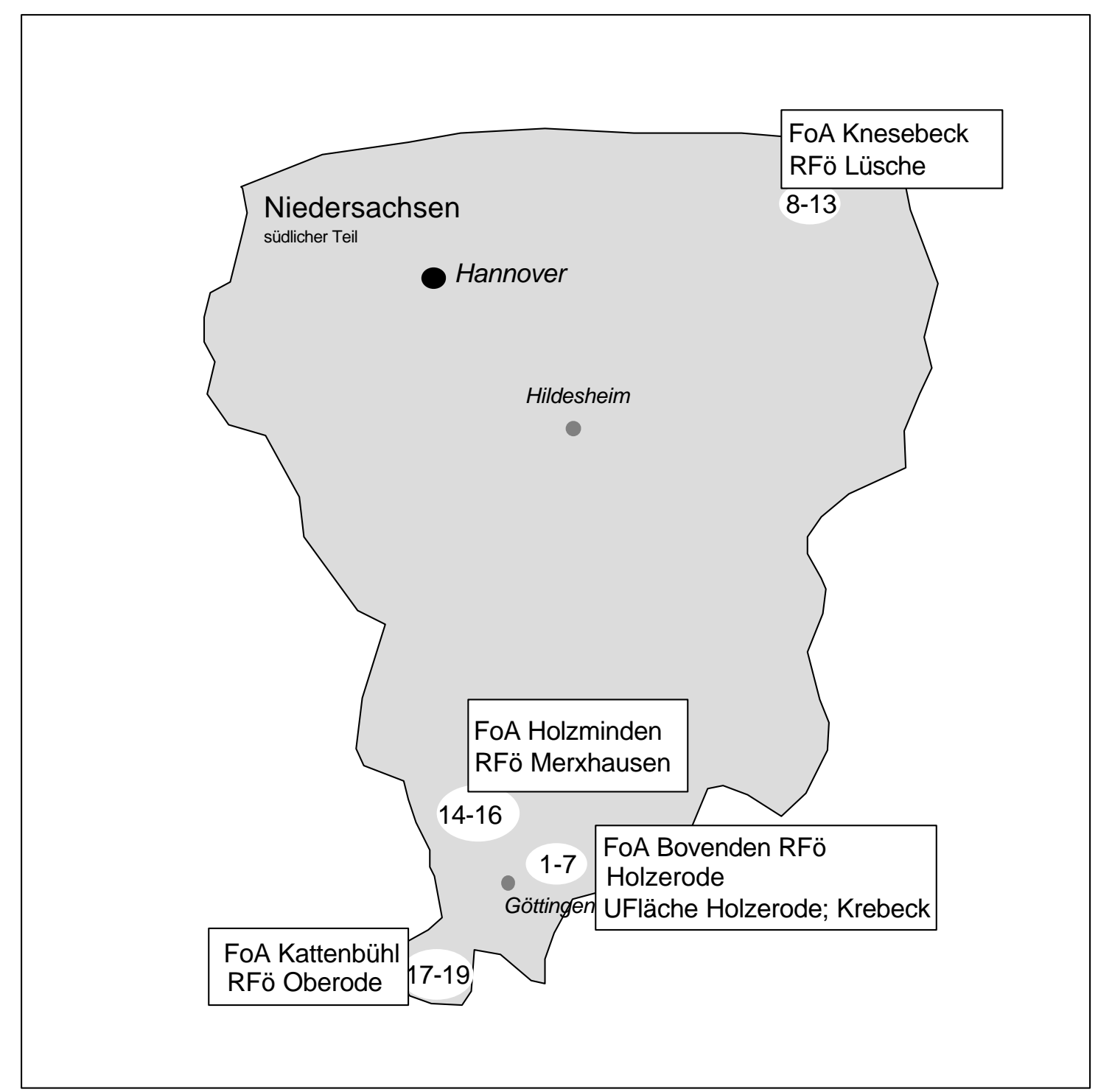

Abb. 1: $\quad$ Lage der Untersuchungsgebiete (Untersuchungsflächen Nr. 1-19)

Einen Überblick über ausgewählte Klimadaten gibt Tabelle 1. 
Tabelle 1: Klimadaten der Untersuchungsflächen (Arbeitskreis Standortskartierung der Arbeitsgemeinschaft Forsteinrichtung; 1985)

\begin{tabular}{|ll|lccc|cc|}
\hline \multirow{2}{*}{$\begin{array}{c}\text { Untersuch- } \\
\text { ungsfläche }\end{array}$} & Wuchsbezirk & \multicolumn{4}{c|}{ Mittlere Temperatur } & \multicolumn{2}{c|}{ Niederschlag } \\
& & Höhe & Jahr & Veg.- & $\mathbf{2 1 0 ^ { \circ } \mathbf { C }}$ & [mm] & mm $]$ \\
NN[m] & {$\left[{ }^{\circ} \mathbf{C}\right]$} & Zeit [ $\left.{ }^{\circ} \mathbf{C}\right]$ & {$[$ Tage $]$} & Jahr & 'eg.zeit \\
\hline Bovenden $_{(1-7)}$ & Göttinger Wald & $201-300$ & 7,8 & 14,0 & 150 & 680 & 340 \\
Knesebeck $_{(8-13)}$ & Hohe Heide & $101-150$ & 8,0 & 14,3 & 148 & 730 & 330 \\
Holzminden $_{(14-16)}$ & Unterer Solling & $250-400$ & 7,5 & 13,4 & 147 & 900 & 420 \\
Kattenbühl $_{(17-19)}$ & Kaufunger Wald & $451-500$ & 7,5 & 14,0 & 150 & 780 & 370 \\
\hline
\end{tabular}

\subsubsection{Beschreibung der Böden}

Tabelle 2 stellt die Bodenverhältnisse der Standorte im Überblick dar. Horizontweise Beschreibungen sind Anhang I (A-B) zu entnehmen.

Tabelle 2: Bodenbeschreibung

\begin{tabular}{|c|c|c|c|c|c|}
\hline Bestand & $\begin{array}{l}\text { Bestand } \\
\text { Nr. }\end{array}$ & $\begin{array}{l}\text { Geolog. Ausgangs- } \\
\text { substrat }\end{array}$ & Bodentyp & $\begin{array}{l}\text { NWK } \\
{[\mathrm{mm}]}\end{array}$ & $\begin{array}{l}\text { Puffer- } \\
\text { bereich }\end{array}$ \\
\hline Holzerode & $\begin{array}{l}1 \\
2-4\end{array}$ & Löß über Buntsandstein & $\begin{array}{l}\text { Pseudogley- } \\
\text { Parabraunerde } \\
\text { (Lehm) }\end{array}$ & 231 & $\begin{array}{l}\text { Aluminium } \\
\text { Silikat }\end{array}$ \\
\hline Krebeck & $\begin{array}{l}5-6 \\
7 \\
\end{array}$ & Löß über Buntsandstein & $\begin{array}{l}\text { Pseudogley- } \\
\text { Parabraunerde } \\
\text { (Lehm) }\end{array}$ & $\begin{array}{r}223 \\
197 \\
\end{array}$ & $\mathrm{Al} / \mathrm{Fe}$ \\
\hline Knesebeck & $\begin{array}{l}8-9 ; 11 \\
10 \\
12-13\end{array}$ & Geschiebesande & $\begin{array}{l}\text { Braunerde-Podsol } \\
\text { (Sand) }\end{array}$ & $\begin{array}{l}144 \\
225\end{array}$ & $\begin{array}{l}\mathrm{Al} / \mathrm{Fe} \\
\text { Eisen } \\
\mathrm{Al} / \mathrm{Fe}\end{array}$ \\
\hline Holzminden & $\begin{array}{l}15 \\
14 \\
16 \\
16\end{array}$ & Löß über Buntsandstein & $\begin{array}{l}\text { Pseudegley- } \\
\text { Parabraunerde } \\
\text { (Lehm) }\end{array}$ & $\begin{array}{l}197 \\
175\end{array}$ & Aluminium \\
\hline Kattenbühl & $\begin{array}{l}17 \\
19 \\
18\end{array}$ & Buntsandstein & Stagnogley & $\begin{array}{l}135 \\
117 \\
121\end{array}$ & $\begin{array}{l}\mathrm{Al} / \mathrm{Fe} \\
\text { Aluminium }\end{array}$ \\
\hline
\end{tabular}

Die wichtigsten Eigenschaften der einzelnen Bodentypen sollen im folgenden kurz beschrieben werden. Die verwendeten Bezeichnungen der standortskundlichen Feuchtestufen und der Horizonte entsprechen den Definitionen der "Bodenkundlichen Kartieranleitung" (AG BODENKUNDE 1982).

\section{Pseudogley-Parabraunerde (Lehm)}

Entscheidend für die räumliche Verbreitung der einzelnen Bodentypen auf den Standorten sind die Verteilung und die Mächtigkeit der quartären Lößablagerungen über dem darunterliegenden Buntsandstein. Die Flächen in Holzerode, Krebeck und Holzminden weisen PseudogleyParabraunerden (Lehm) aus unterschiedlich mächtigen, weitgehend entkalkten Lößen auf. Parabraunerden sind feinkörnige Böden mit einem durch Tonverlagerung an Ton verarmten AHorizont (Al) und einem tonreichen Bt-Horizont. Der wenig wasserdurchlässige Bt-Horizont wirkt mit zunehmendem Tongehalt als Staukörper und führt dann zur Pseudovergleyung mit 
einer wenig tonhaltigen Stauzone (Sw) und einem stark tonhaltigen Staukörper (Sd). Die Humusgehalte der Flächen schwanken zwischen $1 \%$ und 5\% in Holzerode und Holzminden und zwischen 3\% und 8\% in Krebeck. Dabei handelt es sich in Holzerode um mullartigen Moder, in Krebeck um Moder und in Holzminden um f-Mull. Mit $10 \%$ weist der Standort Holzminden bereits im Oberboden hohe Skelettanteile auf. Die Pseudogley-Parabraunerden (Lehm) der Untersuchungsgebiete weisen je nach Mächtigkeit der Böden mit $231 \mathrm{~mm}$ für Holzerode, $197 \mathrm{~mm}$ und $223 \mathrm{~mm}$ für die Standorte in Krebeck und $197 \mathrm{~mm}$ bzw. $175 \mathrm{~mm}$ für Holzminden eine mittlere nutzbare Wasserkapazität (NWK) auf. Die Böden der Standorte Holzerode und Holzminden zeigen jeweils stark unterschiedliche $\mathrm{pH}-$ Werte und liegen damit im Silikat- und im Aluminium Pufferbereich. In Krebeck befinden sich die Böden aller Teilflächen im Aluminium/Eisen-Pufferbereich. Die Flächen in Holzerode wurden 1987 gekalkt. Die Durchwurzelbarkeit kann als gut bezeichnet werden.

\section{Braunerde-Podsol}

Braunerde-Podsole über Geschiebesanden sind auf dem Standort Knesebeck anzutreffen. Dieser Bodentyp ist nach SCHEFFER und SCHACHTSCHABEL (1992) eine typische Weiterentwicklung saurer Braunerden. Die Tonverlagerung kommt durch Freisetzung von Aluminium zustande. Die Horizontabfolge zeichnet sich durch einen schwach gebleichten Ahe-Horizont und einen mit Humus angereicherten und deshalb dunkel gefärbten Bh-Horizont aus. Darauf folgt ein, durch eingelagerte Sesquioxide $(\mathrm{Fe} / \mathrm{Mn})$, mehr oder weniger stark verfestigter Bs-Horizont. Die Humusgehalte im Oberboden liegen im Entwicklungsstadium dieser Böden mit Werten zwischen 4,5\% und 7\% sehr hoch. Die Teilflächen des Standortes unterscheiden sich stark in der Bodenart und weisen daher sehr unterschiedliche nutzbare Wasserkapazitäten (NWK) auf. Die lehmigen Sande einiger Teilflächen führen zu einer NWK von $236 \mathrm{~mm}$, die mittelsandigen Böden dagegen nur zu 144 mm (Anhang I). Die pH-Werte der einzelnen Standorte schwanken zwischen 2,7 und 3,6 und liegen damit im Aluminium/Eisen- bzw. Eisen-Pufferbereich. Die Böden gelten als gut durchwurzelbar.

\section{Stagnogley}

Die Flächen auf dem Standort Kattenbühl können einheitlich dem Bodentyp Stagnogley zugeordnet werden. Diese Böden sind auf Buntsandstein entstanden, der von einer geringmächtigen Fließerdeschicht überlagert ist. Diese tonreiche Fließerde in etwa $60 \mathrm{~cm}$ Tiefe bildet den Stauhorizont, der den Wasserabfluß nach unten verhindert und damit die Vergleyung hervorruft. Auf allen Teilflächen erfolgte eine Bodenbearbeitung vor der Nutzung, weshalb der Ah- durch einen Ap- Horizont ersetzt werden kann. Darunter folgt der für diese Böden typische AhSw mit Eisen- und Mangananreicherungen über dem stark tonigen Sd. Die Teilflächen weisen 3,5\% bis $4 \%$ Ton im Oberboden auf. Die nutzbare Wasserkapazität dieser Böden im effektiven Wurzelraum ist mit Werten zwischen $117 \mathrm{~mm}$ und $135 \mathrm{~mm}$ sehr niedrig. Dies ist jedoch auf die Flachgründigkeit der Durchwurzelungszone zurückzuführen, die durch die Fließerde in $60 \mathrm{~cm}$ Tiefe begrenzt wird (Anhang I). 


\subsection{Untersuchungsbestände}

\subsubsection{Vorbemerkungen}

Die Bestände werden im folgenden nicht weiter numeriert, sondern mit dem Namen des jeweiligen Forstamtes betitelt. Eine Übersicht der Untersuchungsflächen gibt Tabelle 3. In Holzerode geben die Bezeichnungen über die Mischungsverhältnisse, in Krebeck und Knesebeck über das Alter der Bestände Aufschluß. In Holzminden und Kattenbühl werden durch die Bezeichnungen die Verjüngungsmethoden charakterisiert.

\section{Tabelle 3: Probeflächen}

\begin{tabular}{|c|c|c|c|c|c|c|c|c|}
\hline FoA & Nr. & Fläche & Bezeichnung & $\begin{array}{l}\text { Zielset- } \\
\text { Zung }\end{array}$ & FoA & $\mathrm{Nr}$ & Bezeichnung & $\begin{array}{l}\text { Ziel- } \\
\text { :etzung }\end{array}$ \\
\hline \multirow{5}{*}{$\begin{array}{l}\frac{c}{0} \\
\frac{0}{0} \\
\frac{5}{0} \\
\partial \\
\varnothing\end{array}$} & $\begin{array}{l}1 \\
2 \\
3\end{array}$ & $\begin{array}{l}\text { Holzerode } \\
\text { Holzerode } \\
\text { Holzerode }\end{array}$ & $\begin{array}{l}\text { Buche } \\
\text { Buche / Fichte } \\
\text { Fichte }\end{array}$ & \multirow{2}{*}{ 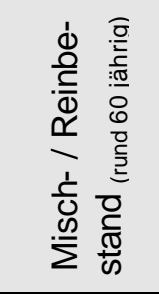 } & Knesebeck & $\begin{array}{l}11 \\
12 \\
13\end{array}$ & $\begin{array}{l}\text { Kiefer } 20 \text { jähr. } \\
\text { Kiefer } 33 \text { jähr. } \\
\text { Kiefer } 47 \text { jähr. }\end{array}$ & 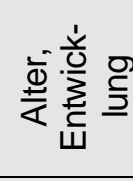 \\
\hline & 4 & Holzerode & Fichte / Buche & & \multirow{4}{*}{$\begin{array}{l}\text { Holzminden } \\
\text { Eiche } \\
22 \text { jährig }\end{array}$} & \multirow{4}{*}{$\begin{array}{l}14 \\
15 \\
16\end{array}$} & \multirow{4}{*}{$\begin{array}{l}\text { Pflanzung } \\
\text { Saat } \\
\text { Verpflanzung }\end{array}$} & \multirow{4}{*}{ 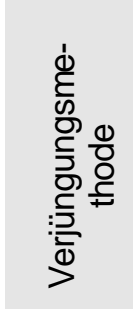 } \\
\hline & 5 & Krebeck & Fichte 21 jähr. & & & & & \\
\hline & 6 & Krebeck & Fichte 30 jähr. & एั & & & & \\
\hline & 7 & Krebeck & Fichte 41 jähr. & $\frac{\bar{\Phi}}{\frac{1}{4}} \cdot \frac{0}{3}$ & & & & \\
\hline \multirow{3}{*}{ 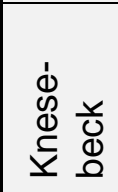 } & 8 & & Fichte 20 & \multirow{3}{*}{ 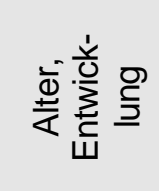 } & \multirow{3}{*}{$\begin{array}{l}\text { Kattenbühl } \\
\text { Eiche } \\
13 \text { jährig }\end{array}$} & 17 & Pflanzung & \multirow{3}{*}{ 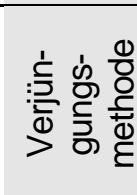 } \\
\hline & 9 & & Fichte 31 & & & 18 & Saat I & \\
\hline & 10 & & Fichte 50 & & & 19 & Saat II & \\
\hline
\end{tabular}

\section{Eiche}

Für die Untersuchungen an Eiche werden Flächen gewählt, die nach dem Betriebswerk mit Traubeneichen bestockt sind. Die Unterscheidung von Stiel- und Traubeneiche ist nicht immer eindeutig möglich. Aufgrund der Hybridbildung von Quercus robur L. und Quercus petraea (Matt.) Liebl. und weil gegensätzliche Ausprägungen von Merkmalen zwischen den Typen fehlen, halten es KLEINSCHMIT et al (1995) für angebracht, von einer biologischen Art (Quercus robur L. ssp. Petraea Kl. et Kr. et Rol.) zu sprechen (ROLOFF UND BÄRTELS 1996).

Nach KRAHL-URBAN (1951) und KREUTZER (1961) besteht darüber hinaus zwischen dem Wurzelsystem der Stiel- und dem der Traubeneiche kein Unterschied, so daß in dieser Arbeit keine Differenzierung nach Trauben- und Stieleiche vorgenommen wird. 


\subsubsection{Untersuchungsgebiet Bovenden (ehemals Radolfshausen), Fichte und Buche}

\subsubsection{Untersuchungsgebiet Holzerode}

Aus rund 30 ha Fläche der Abteilungen 315 / 316 und 317 wurden 40 Probebäume entnommen. Es soll hier die Fragestellung bearbeitet werden, ob sich die Grobwurzelarchitektur von Buche und Fichte in Mischung von der im Reinbestand erwachsenen Buche oder Fichte unterscheidet. Da keine dieser Aufgabenstellung entsprechenden benachbarte Bestände mit vergleichbaren Standortverhältnissen gefunden wurden, wurde diese sehr große Fläche gewählt. Sie wies in ihrem Kern von 11 ha die geforderten Reinbestandsmerkmale auf. Aus dieser Fläche wurden je 10 Fichten und Buchen entnommen. Im umliegenden Bereich konnten je 10 Buchen und Fichten gefunden werden, die von mindestens fünf Bäumen einer anderen Baumart umgeben waren.

Die Probebäume der Kollektive Buche / Fichte, Fichte und Fichte / Buche wurden aus einem Fichten/ Buchen Mischbestand entnommen, der sich folgendermaßen beschreiben läßt: Fichte, 47 jähriges, geringes bis mittleres Baumholz aus Pflanzung, wüchsig, langschaftig, kleinkronig mit stamm bis flächenweise eingemischter Buche, 57 jährig, geringes Baumholz aus Naturverjüngung und Pflanzung, gleichwüchsig, mittel- und geradschaftig, großkronig mit vereinzelten, stammweise eingemischten Europäischen Lärchen, Birken, Kiefern, Bergahornen und Eichen, locker bis geschlossen. Die Probebäume der Kollektive Buche, Buche/Fichte und Fichte / Buche stammen aus einem Buchenbestand mit 0,6 ha Fichtenbeimischung: Buche, 75 jährig ( \pm 20 Jahre), geringes bis mittleres Baumholz aus Naturverjüngung, wüchsig, langschaftig, mittelkronig mit stamm- bis gruppenweise eingemischter Europäischer Lärche und Fichte, 62 jährig, geringes bis mittleres Baumholz aus Pflanzung, gleichwüchsig und kleinkronig mit stammbis truppweise beigemischter Birke, Eiche, Kirsche und Bergahorn.

\subsubsection{Untersuchungsgebiet Krebeck}

In Krebeck wurden drei nebeneinander liegende Fichtenprobeflächen im Alter von 21, 30 und 41 Jahren aus den Abteilungen 301 und 302 gewählt. Die Feinerschließung wurde bei den älteren Beständen 1994 durchgeführt. Bei der jüngeren Fläche hat noch keine Feinerschließung stattgefunden. Die Rückegassenabstände betragen $25 \mathrm{~m}$. Die Positionen der gewählten Probebäume sind der Skizze (Abb. 2) zu entnehmen. 


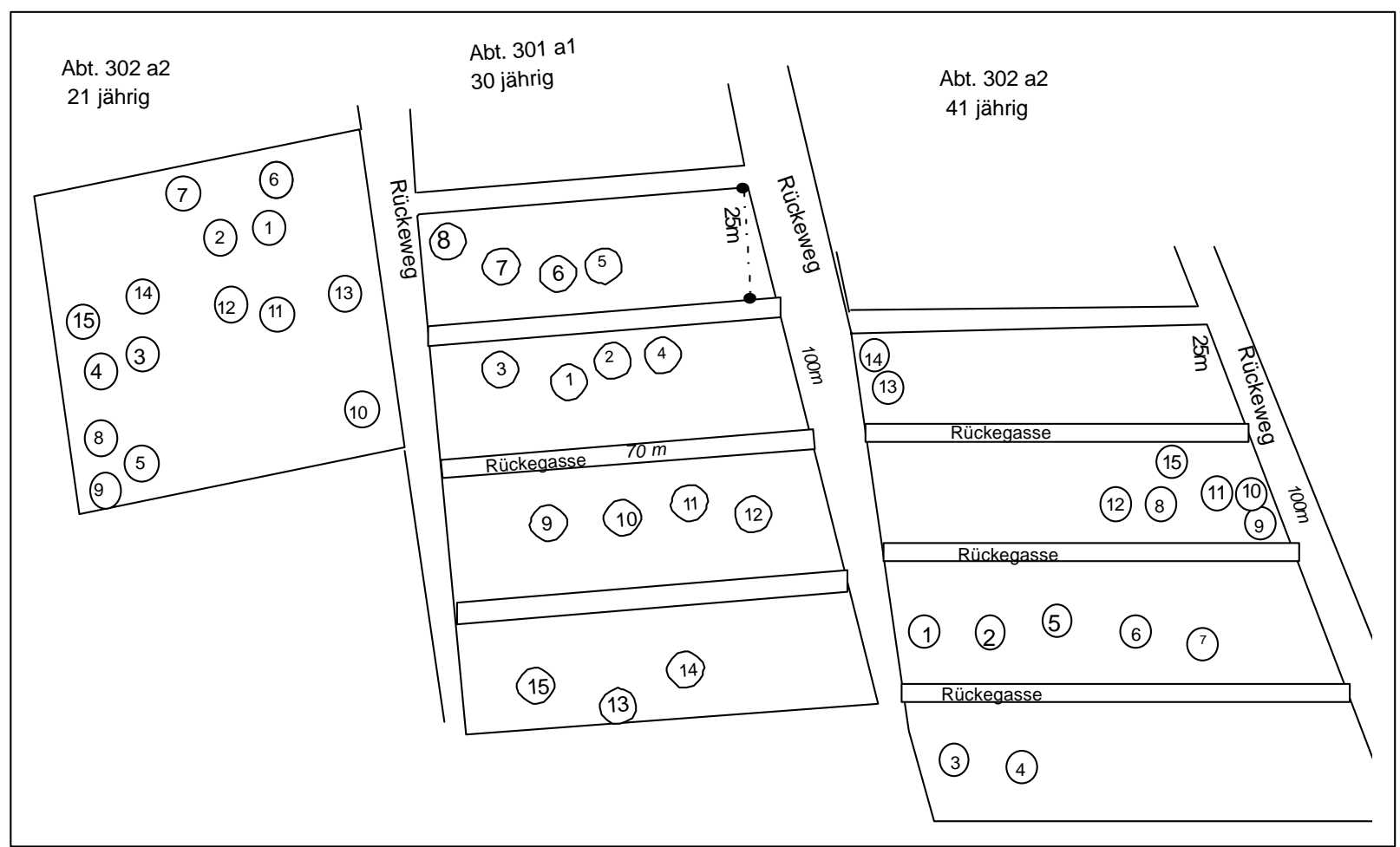

Abb. 2: $\quad$ Skizze der Untersuchungsgebiete Krebeck, 21-, 30- und 41 jährige Fichte

Bestandesbeschreibende Daten sind in Tabelle 4 dargestellt. Alle Flächen weisen eine bessere als erste Ertragsklasse auf. Der Bestockungsgrad der beiden jüngeren Flächen läßt auf einen guten Pflegezustand schließen. Die Läuterungen und Durchforstungen fanden in ausreichendem Maße statt. Einzelstammweise sind in allen Beständen Eiche, Bergahorn, Birke und Buche beigemischt, die begünstigt wurden.

Tabelle 4: Bestandesbeschreibung Krebeck

\begin{tabular}{|l|c|c|c|}
\hline & Fichte 21 & Fichte 30 & Fichte 41 \\
\hline Alter (Jahre) & 21 & 30 & 41 \\
\hline Natürliche Altersstufe & Stangenholz & geringes Baumholz & geringes Baumholz \\
\hline Begründung & $\begin{array}{c}\text { 1976 Pflanzung } \\
\text { 2+0 Westerhof 03 }\end{array}$ & $\begin{array}{c}19702+2 \text { Westerhof } \\
633.081,7 \mathrm{~m}^{2} \text { Ver- } \\
\text { band 3400 Pfl./ha }\end{array}$ & $\begin{array}{c}\text { Pflanzung 1958; } \\
2+0\end{array}$ \\
\hline Stammzahl St/ha & 2833 & 2373 & 2091 \\
\hline Schaftlänge & mittelschaftig & mittel- langschaftig & mittel- langschaftig \\
\hline Bestockungsgrad $\left(\mathrm{B}^{\circ}\right)$ & 1,0 & 0,8 & 1,1 \\
\hline Höhe Zentralbaum $(\mathrm{m})$ & 10,5 & 14,1 & 18,2 \\
\hline BHD Zentralbaum $(\mathrm{cm})$ & 12,5 & 14,5 & 15,5 \\
\hline Vorrat fm / ha & 100 & 202 & 350 \\
\hline Durchforstungen & Läuterung 1987 & $\begin{array}{c}\text { Läuterung 1987, } \\
\text { Durchforstung1994 }\end{array}$ & $\begin{array}{c}\text { Keine Läuterung } \\
\text { Schneebruch '85/'86 } \\
\text { Durchforstung 1992 }\end{array}$ \\
\hline Vorbestand & & Fichte & Fichte \\
\hline Hangneigung $\left(^{\circ}\right)$ & Fichte & 2 & 4 \\
\hline Nat. Waldgesellschaft & Luzulo Fagetum & Luzulo Fagetum & Luzulo Fagetum \\
\hline
\end{tabular}




\begin{tabular}{|l|c|c|c|}
\hline & Fichte 21 & Fichte 30 & Fichte 41 \\
\hline Alter (Jahre) & 21 & 30 & 41 \\
\hline $\begin{array}{l}\text { Aktuelle Waldgesell- } \\
\text { schaft }\end{array}$ & Culto Picetum & Culto Picetum & Culto Picetum \\
\hline Flächengröße (ha) & 1 & 7,4 & 5,6 \\
\hline Probeflächengröße (ha) & 0,8 & 1 & 1 \\
\hline
\end{tabular}

\subsubsection{Untersuchungsgebiet Knesebeck, Kiefer und Fichte}

Aus der Abteilung 93 der Revierförsterei Knesebeck wurden 20- und etwa 50 jährige und aus den Abteilungen 82 und 7530 jährige Probebäume entnommen. Bestandesbeschreibende Daten sind nur für die älteren Flächen in Tabelle 5 dargestellt. Die 20 jährigen Fichten und Kiefern wurden aus einem mehrschichtigen Mischbestand, bestehend aus 112 jähriger Kiefer als Überhalt mit locker bis räumdiger Beimischung 44 jähriger Kiefern und einzelstammweiser Mischung mit Buche $\left(\mathrm{B}^{\circ} 0,5\right)$ entnommen. Der Nachwuchs, aus dem die Probebäume stammen, besteht aus ca. 20 jähriger, natürlich verjüngter Kiefer und horstweiser, gepflanzter, 20 jähriger Fichte $\left(\mathrm{B}^{\circ} 0,7\right)$ (Hohlspatenpflanzung mit 0+1+2 Westerhof 13004 x $\left.1 \mathrm{~m}\right)$.

Tabelle 5: Bestandesbeschreibung Knesebeck

\begin{tabular}{|l|c|c|c|c|}
\hline Bestand & Fichte & Fichte & Kiefer & Kiefer \\
\hline Alter (Jahre) & 31 & 50 & 33 & 47 \\
\hline Natürliche Altersstufe & $\begin{array}{c}\text { Schwaches } \\
\text { Baumholz }\end{array}$ & $\begin{array}{c}\text { Mittleres Baum- } \\
\text { holz }\end{array}$ & $\begin{array}{c}\text { Schwaches } \\
\text { Baumholz }\end{array}$ & $\begin{array}{c}\text { Mittleres } \\
\text { Baumholz }\end{array}$ \\
\hline Begründung & $\begin{array}{c}\text { Holhlspaten- } \\
\text { pflanzung 2+1; } \\
4348 \text { St, 2x } \\
1,15 m \text { 1965 } \\
\text { Nachbesserung } \\
1600 \text { St 2+2 } \\
\text { Nordd.Tiefland, } \\
300 \text { 2+1 We- } \\
\text { sterhöfer 1,5x2 }\end{array}$ & $\begin{array}{c}\text { Nicht bekannt, } \\
\text { da zwischen } \\
\text { dazugekauft }\end{array}$ & $\begin{array}{c}\text { Pflanzung Ro- } \\
\text { meverfahren }\end{array}$ & Saat \\
\hline Stammzahl St/ha & 2150 & 1375 & 2700 & \\
\hline Schaftlänge & mittelschaftig & mittelschaftig & langschaftig & langschaftig \\
\hline Bestockungsgrad $\left(\mathrm{B}^{\circ}\right)$ & 0,8 & 0,8 & 1,1 & 0,8 \\
\hline Höhe Zentralbaum $(\mathrm{m})$ & 13,8 & 20,3 & 12,4 & 18,1 \\
\hline BHD Zentralbaum $(\mathrm{cm})$ & 13,1 & 21 & 11,9 & 19,2 \\
\hline Vorrat fm/ha & 190 & 320 & 143 & 214 \\
\hline Durchforstungen & $\begin{array}{c}\text { Entnahme von } \\
\text { Bäumen zum } \\
\text { Zaunbau }\end{array}$ & Nicht bekannt & Nicht bekannt & $\begin{array}{c}198324 \\
\text { fm/ ha Jung- } \\
\text { durch-forstung }\end{array}$ \\
\hline Vorbestand & Kiefer & Nicht bekannt & Kiefer & Kiefer \\
\hline Hangneigung $\left(^{\circ}\right)$ & 0 & 0 & 0 & 0 \\
\hline Reihenkoordinaten & Nord-Süd & Ost-West & Ost-West & Ost-West \\
\hline Nat. Waldgesellschaft & Luzulo Fagetum & Luzulo Fagetum & Luzulo Fagetum & Luzulo \\
\hline
\end{tabular}




\begin{tabular}{|l|c|c|c|c|}
\hline Bestand & Fichte & Fichte & Kiefer & Kiefer \\
\hline Alter (Jahre) & 31 & 50 & 33 & 47 \\
\hline Akt. Waldgesellschaft & Culto Picetum & Culto Picetum & Culto Pinetum & $\begin{array}{c}\text { Fagetum } \\
\text { Culto Pinetum }\end{array}$ \\
\hline Flächengröße (ha) & 1,1 & 1,3 & 2,2 & 17,3 \\
\hline Probeflächengröße (ha) & 1,1 & 1,3 & 0,7 & 1,0 \\
\hline
\end{tabular}

\subsubsection{Eiche}

\subsubsection{Untersuchungsgebiet Holzminden}

Für diese Untersuchungen wurden aus der Abteilung 50 (5,6 ha) der Revierförsterei Merxhausen drei Bestände ausgewählt. Sie werden in Tabelle 4 beschrieben. Die Untersuchungsflächen liegen innerhalb eines langgezogenen, ca.100 m schmalen, $600 \mathrm{~m}$ langen Streifens in der Reihenfolge Saat-Pflanzung-Verpflanzung in West-Ost Richtung (Abb. 3). Bei der Verpflanzung handelt es sich um aus dem Saatbestand entnommene und in diese Fläche verpflanzte Eichen. Die Reihen der Saateichen verlaufen etwa in West-Ost-, die der Pflanz- und Verpflanzungseichen in Nord-Süd-Richtung.

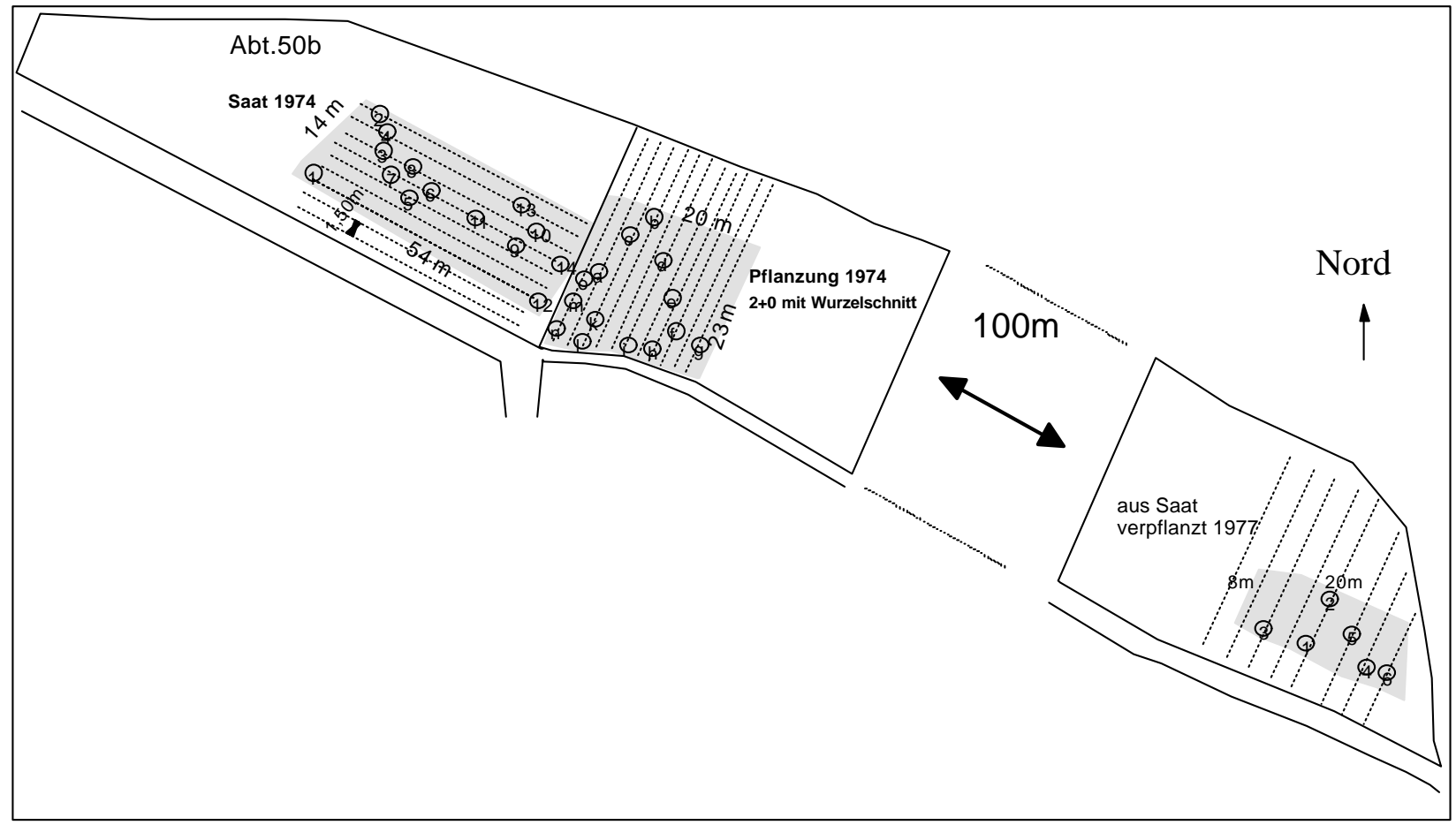

Abb. 3: Schematische Darstellung der Probeflächen Holzminden, 22 jährige Eiche, Pflanzung, Saat und Verpflanzung

Die drei Eichenbestände unterscheiden sich im Alter und nach Beschreibung im Bestandeslagerbuch kaum. Sie differieren bei genauerer Aufnahme jedoch in einem nicht geringen Maße. Die Saatfläche weist als dienende Baumart allein Hainbuche auf und zeigt sich wüchsiger als die beiden anderen Flächen. Die Pflanzung hat einen erheblichen Anteil (38\%) an Mischbaumarten wie Roteiche (Quercus rubra L.) und Linde (Tilia cordata MILL.). Die Linde wurde 1994 geköpft. Bei der Verpflanzung haben Roteiche und Linde einen höheren Mischungsanteil als bei der Pflanzung. Darüber hinaus ist die Linde in diesem Bestand nicht geköpft worden, so 
daß beide Baumarten in den Hauptbestand eingewachsen sind und ihn teilweise schon überwachsen haben.

Tabelle 6: Bestandesbeschreibung Holzminden

\begin{tabular}{|c|c|c|c|}
\hline Eiche & Pflanzung & Saat & $\begin{array}{c}\text { Verpflanzung aus } \\
\text { Saat }\end{array}$ \\
\hline Alter (Jahre) & 24 & 22 & 22 \\
\hline Natürliche Altersstufe & Stangenholz & Stangenholz & Stangenholz \\
\hline Begründung & Pflanzung $19742+0$ & $\begin{array}{c}\text { Saat } 1974500 \\
\text { kg/ha Herkunft } \\
\text { Pfälzer Wald / O- } \\
\text { denwald }\end{array}$ & $\begin{array}{c}\text { Herkunft Saat 74, } \\
\text { umgesetzt } 1977\end{array}$ \\
\hline Stammzahl & 3400 & 4500 & 4100 \\
\hline Schaftlänge & langschaftig & langschaftig & langschaftig \\
\hline Bestockungsgrad $\left(\mathrm{B}^{\circ}\right)$ & 1,0 & 1,1 & 1,0 \\
\hline Höhe Zentralbaum (m) & 11,1 & 12,2 & 10,4 \\
\hline BHD Zentralbaum (cm) & 9,9 & 13,5 & 8,4 \\
\hline Maßnahmen & keine & keine & keine \\
\hline Vorbestand & Wiesenaufforstung & Ackeraufforstung & Wiesenaufforstung \\
\hline Hangneigung $\left(^{\circ}\right)$ & 2 & 2 & 3 \\
\hline Waldgesellschaft & $\begin{array}{l}\text { Galio Fagetum dry- } \\
\text { opteridetosum }\end{array}$ & $\begin{array}{c}\text { Galio Fagetum } \\
\text { dryopteridetosum }\end{array}$ & Galio Fagetum \\
\hline Mischbaumarten & $\begin{array}{l}\text { Linde geköpft 1995; } \\
\text { REiche ins. } 38 \% \\
\text { BHD } 6 \text { cm Höhe } 9 \text { m }\end{array}$ & $\begin{array}{c}\text { Hainbuche } 7 \% \\
\text { BHD } 3 \mathrm{~cm} \\
\text { Höhe } 5,3 \mathrm{~m}\end{array}$ & $\begin{array}{c}\text { Wi- Linde, REiche } \\
53 \% \text { BHD } 8,5 \mathrm{~cm} \\
\text { Höhe } 11 \mathrm{~m}\end{array}$ \\
\hline Flächengröße (ha) & 2,4 & 1,8 & 1,4 \\
\hline Probeflächengröße (ha) & 0,1 & 0,1 & 0,5 \\
\hline
\end{tabular}




\subsubsection{Untersuchungsgebiet Kattenbühl}

Auf einer rund 24 ha großen Windwurffläche wurden vom Forstamt Kattenbühl, Revierförsterei Oberode, verschiedene Eichenversuche angelegt. Unter anderem ein gepflanzter und zwei gesäte Bestände, die für die Fragestellung dieser Arbeit geeignet waren. Bestandesbeschreibende Daten sind Tabelle 7 zu entnehmen. Der Aufbau dieser Flächen ist in Abb. 4 skizzenhaft dargestellt.

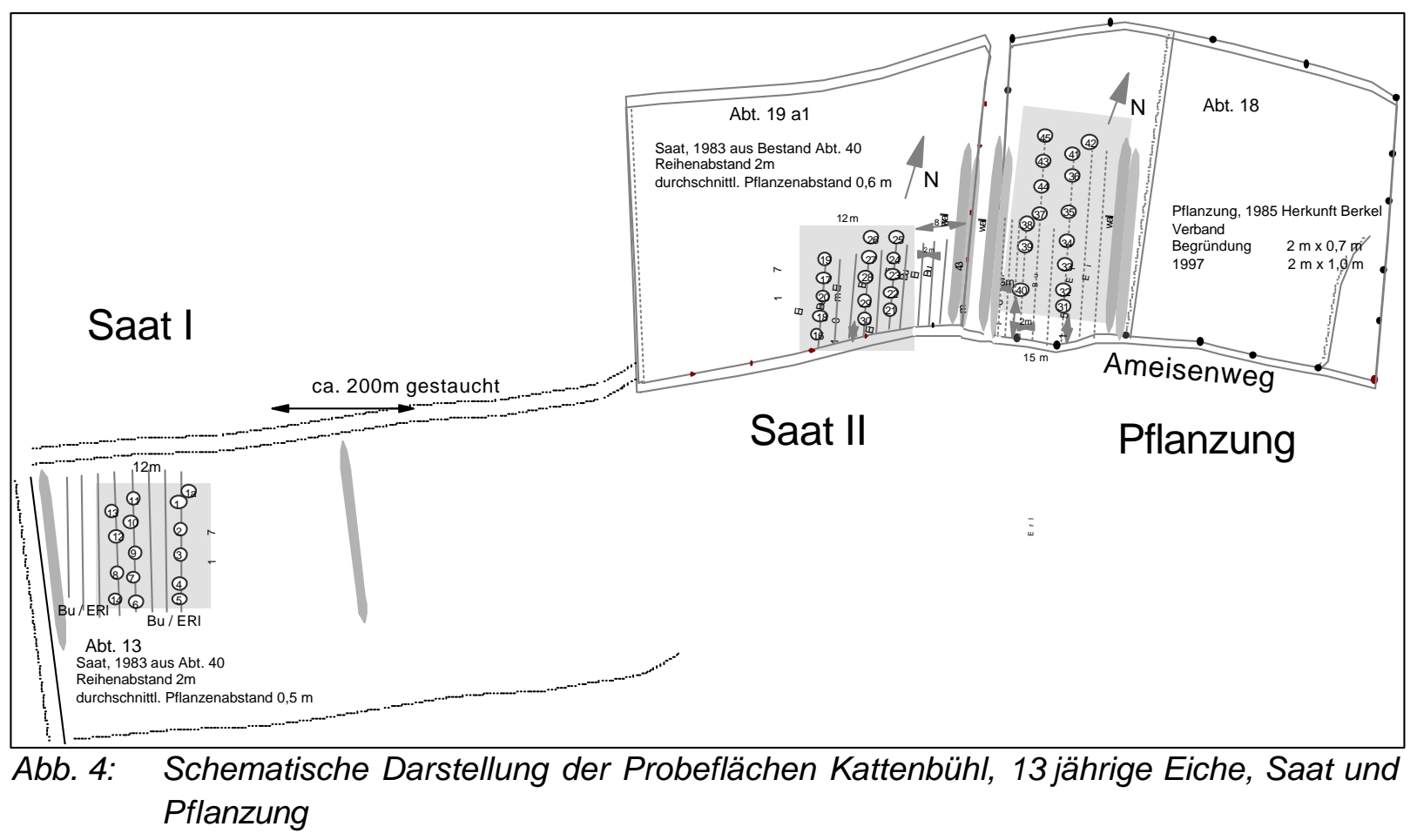

Nach Schlagräumung, Kalkung und Schaffung von Dämmen (Dämme-Rome) auf Kahlschlagflächen wurde mit Eiche, sowohl Saat als auch Pflanzung, in reihenweiser Mischung mit Buche aufgeforstet. Zum Zeitpunkt der Untersuchungen befanden sich die drei ausgewählten Flächen in der Dickungsphase. Durch seine Wüchsigkeit ragt der gepflanzte Bestand deutlich hervor. Ebenso unterscheiden sich die beiden Saatbestände gleichen Alters und gleicher Herkunft in ihrem Wachstum. Die Saat I - Fläche ist wüchsiger als die Saat II - Fläche. Da aus der ersten Fläche mehr Wildlinge geworben wurden als aus der zweiten Fläche, hatte die Eiche des Saat I-Bestandes einen größeren Standraum zur Verfügung. Begünstigt durch mehr Licht und Nährstoffe konnten die Eichen der Saat I Fläche so dicker werden. Darüber hinaus sind sie auch höher. Geographisch liegen die beiden Bestände ca. 200 m auseinander (Abb. 4). 
Tabelle 7: Bestandesbeschreibung Kattenbühl

\begin{tabular}{|l|c|c|c|}
\hline Eiche & Pflanzung & Saat I & Saat II \\
\hline Alter & 14 & 13 & 13 \\
\hline Natürliche Altersstufe & Dickung & Dickung & Dickung \\
\hline Begründung & $\begin{array}{c}\text { 1985 Schlagräumung } \\
\text { u. Kalkung 5t/ha } \\
\text { Dämme -Rome; } \\
\text { Pflanzung 1985 2+1 } \\
\text { Berkel 818.07 10/50 }\end{array}$ & $\begin{array}{c}\text { 1983 Stockrodung u. } \\
\text { Kalkung 2t/ha Däm- } \\
\text { me -Rome; Saat } \\
1983\end{array}$ & $\begin{array}{c}\text { 1983 Stockrodung u. } \\
\text { Kalkung 2t/ha Däm- } \\
\text { me -Rome; Saat } \\
\text { FoA Kattenbühl } \\
1983 \\
\text { 150kg auf 0,3 ha } \\
\text { Herkunft Abt. 40 FoA } \\
\text { Kattenbühl }\end{array}$ \\
\hline Düngung & $\begin{array}{c}\text { 1985 Einzelpflanzen- } \\
\text { düngung Volldünger; } \\
\text { Lupinenaussaat 1985 }\end{array}$ & $\begin{array}{c}\text { Einzelpflanzen- } \\
\text { düngung '86/'87 }\end{array}$ & $\begin{array}{c}\text { Einzelpflanzen- } \\
\text { düngung '86/'87 }\end{array}$ \\
\hline Stammzahl St/ha & 5000 & 8300 & 10000 \\
\hline Schlußgrad & geschlossen & geschlossen & gedrängt \\
\hline Höhe Zentralbaum( m) & 6,3 & 5,8 & 5,4 \\
\hline BHD Zentralbaum(cm) & 6 & 5,6 & 5,0 \\
\hline Jungwuchspfege & Jungwuchspflege '91 & Jungwuchspflege '91 & keine \\
\hline Vorbestand & Fichte & Fichte & Fichte \\
\hline Hangneigung & Plateau & Plateau & Plateau \\
\hline Waldgesellschaft & Galio Fagetum & Galio Fagetum & Galio Fagetum \\
\hline Mischbaumarten & $\begin{array}{c}\text { Bu/RErle/BAh/Bi'87/R } \\
\text { Erl 90 geköpft }\end{array}$ & $\begin{array}{c}\text { Bu/BAh / Bi / } \\
\text { RErl '87/'90 geköpft }\end{array}$ & $\begin{array}{c}\text { Bu/BAh/Bi/RErl } \\
\text { RErl '87/'90 geköpft }\end{array}$ \\
\hline Flächengröße (ha) & 3,2 & 2 & 0,3 \\
\hline $\begin{array}{l}\text { Probeflächengröße } \\
\text { (ha) }\end{array}$ & 0,25 & 0,25 & 0,25 \\
\hline
\end{tabular}




\section{Methoden}

\subsection{Bodenanalyse}

Im frischen Zustand wurden im Labor die $\mathrm{pH}\left(\mathrm{H}_{2} \mathrm{O}\right)$ - und $\mathrm{pH}(\mathrm{KCl})$ - Werte der Bodenlösung anhand einer Mischprobe aus zwei Probenahmen ermittelt. Nach Trocknung bei $40^{\circ} \mathrm{C}$ wurden im Perkolationsverfahren mit $\mathrm{NH}_{4} \mathrm{Cl}$ die effektive Austauschkapazität (Ake) und die austauschbaren Kationen (K, Ca, Mg, Fe und Al), wie bei MeIwES (1984) beschrieben, bestimmt. Die Ergebnisse der Bodenanalysen sind in Anhang I (C-F) zu finden. Die feldbodenkundlichen Aufnahmen wurden in Anlehnung an die forstliche Standortaufnahme 1996 (ARBEITSGEMEINSCHAFT FORSTEINRICHTUNG 1996) durchgeführt.

\subsection{Auswahl und Erfassung der Probebäume}

Aus jeder Versuchsfläche wurden zwischen sechs und 16 Bäume aus dem Bestandesinneren ausgewählt. Es wurde in jedem Bestand eine etwa gleich große Anzahl Bäume der ersten vier Kraft'schen Klassen (3-7 Bäume) entnommen. Durch die Auswahl der verschiedenen Kraft'schen Klassen wurde erreicht, daß die Baumdurchmesserverteilung des Bestandes weitgehend abgedeckt ist. Weiterhin sollten die Konkurrenzverhältnisse, die durch die soziale Stellung beschrieben werden, erfaßt werden.

Für die Mischbestandsuntersuchungen wurden die Probebäume so ausgewählt, daß der über alle Bäume gemittelte BHD etwa gleich war.

Am stehenden Baum wurden in 1,30 m Höhe die Durchmesser (BHD) ermittelt. Weiterhin wurde die Nordrichtung und der Stammfuß gekennzeichnet. So waren nach der Baumentnahme an den freiliegenden Wurzelsystemen Himmelsrichtung und Wurzelansatz noch erkennbar.

\subsection{Konkurrenzbedingungen}

Der Konkurrenzdruck, dem ein einzelner Baum ausgesetzt ist, ergibt sich aus den Dimensionen benachbarter Bäume und den Abständen zwischen Probebaum und Konkurrent (ToMÉ und BURKHART 1986, HolmeS und REED 1991, BIGING und DOBBERTIN 1992).

Bei allen Flächen wurden die drei nächsten Nachbarn der Probebäume mit ihrem Durchmesser und dem Abstand zum Probebaum aufgenommen.

Allein in Holzerode wurden weiterhin die Höhen der fünf bis neun Nachbarn ermittelt, so daß der im folgenden erörterte HEGYI - Index (1974) und der Überragungsindex (HARTKOPF 1994, BEHLEN 1996, BIRKE 1997 und BOMMER 1998) angewendet werden können.

\subsubsection{HEGYI-Index}

HEGYI (1974) hat einen Index (Formel 1) erstellt, der den BHD eines Konkurrenten mit dem Abstand des Probebaumes zum Konkurrenten gewichtet. Je größer der berechnete Wert, desto größer ist die Konkurrenz einzuschätzen. Für wenig bedrängte Bäume liegt der HEGYI-Index (C) bei eins, für stark bedrängte kann er in seltenen Fällen bis 15 ansteigen. 


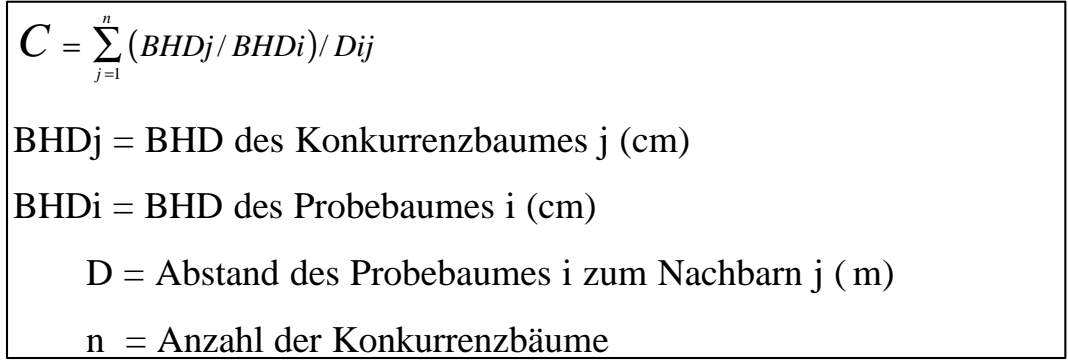

Formel 1: HEGYI-Index (1974)

Dabei stellt sich die Frage, welcher Nachbarbaum wirklich als Konkurrent anzusehen ist. HEGYI (1974) definiert für die Entfernung Probebaum/Konkurrent einen konstanten Radius von 3,05 m. In diesem Umkreis geht jeder Baum in die Berechnung des Indexes ein. Mit anehmendem Alter steigt jedoch der Baumabstand, sei es durch Durchforstungen oder durch natürliche Selektion. Dies hat für die Aussagekraft des Indexes den Nachteil, daß der ermittelte Wert abnimmt und so einen geringen Konkurrenzdruck dokumentiert. Somit wird die Bedrängung nicht mehr hinreichend genau beschrieben.

Aus diesem Grund wurde von BIGING und DoBBERTIN 1992 eine Formel entwickelt, mit der, abhängig von Abstand und BHD der Probe- und Nachbarbäume, eine Konkurrenzsituation nachgewiesen werden kann (Formel 2).

\begin{tabular}{|l|}
$\mathrm{Dt}<(\mathrm{BHDp}+\mathrm{BHDn}) / 8$ \\
$\mathrm{Dt} \quad=$ Abstand des Probebaumes zum Nachbarn $(\mathrm{m})$ \\
$\mathrm{BHDp}=\mathrm{BHD}$ des Probebaumes $(\mathrm{cm})$ \\
$\mathrm{BHDn}=\mathrm{BHD}$ des Nachbarbaumes $(\mathrm{cm})$ \\
Formel 2: \\
Berechnung des relevanten Konkurrenten nach \\
BIGING und DOBBERTIN (1992)
\end{tabular}

Nach Bestimmung der jeweiligen Konkurrenten werden deren Daten für die Berechnung der Konkurrenz nach HEGYI (1974) verwendet.

\subsection{2 Überragungswinkel}

Eine weitere Möglichkeit zur Beschreibung der Konkurrenzsituation stellt ein Höhenvergleich zwischen Probebaum und Nachbarbäumen dar. Für die Fichten und Buchen aus Holzerode wurden alle Höhen der Nachbarbäume ermittelt, die die Krone des Probebaumes berührten. Von der Höhe und der Entfernung dieser benachbarten Bäume hängt es ab, inwiefern der Zentralbaum beschattet ist und / oder selbst für seine Nachbarn beeinträchtigende Schattenbildungen hervorruft (SCHMALTZ und MERKEL 1995). Beide Kriterien werden in dem "Überragungswinkel" (HARTKOPF 1994, BEHLEN 1996, BIRKE 1997 und BOMMER 1998) zur Bestimmung der Konkurrenzsituation genutzt (Abb. 5). Mit Zunahme der Beschattung wird das Assimilationsvermögen und damit die Wuchskraft eines Baumes eingeschränkt. Der Vorteil des Überragungsindexes gegenüber anderen sich auf die Baumhöhe beziehenden Konkurrenzindizes liegt in der leichten Herleitung (Formel 3). 


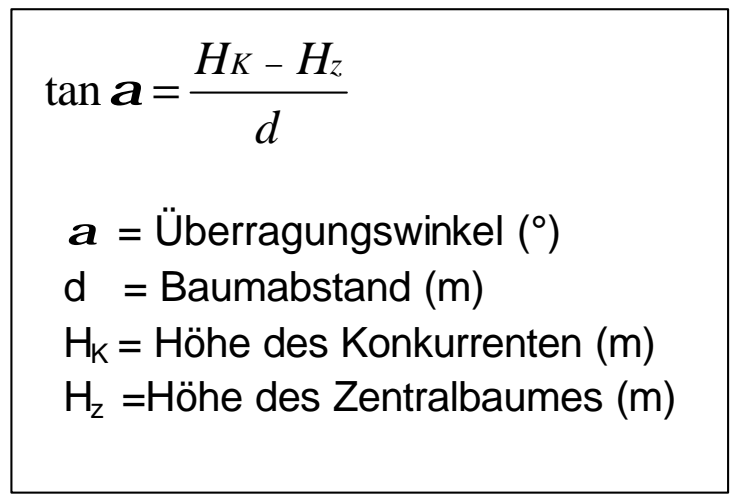

Formel 3: Überragungswinkel ( $\alpha$ ) (u.a. HARTKOPF 1994)

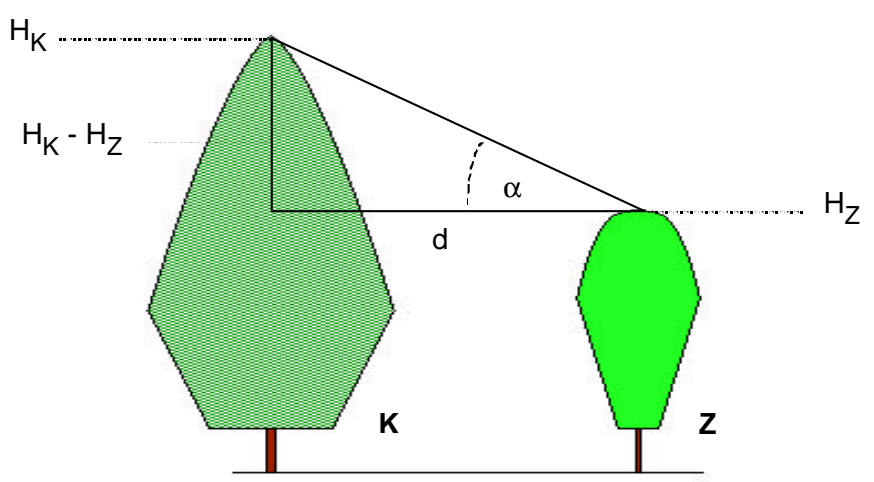

Abb. 5: Darstellung zur Berechnung des Überragungswinkels $(\alpha)$

Für den einzelnen Nachbarn können Werte von minimal $0^{\circ}$ mit wenig Konkurrenz (gleiche Höhen, große Abstände) und der Annäherung an $90^{\circ}$ bei erheblicher Höhenüberlegenheit und Nähe des Konkurrenten zum Zentralbaum vorkommen. Die Summe der Winkel $(\boldsymbol{\alpha})$ aller aufgenommenen Nachbarbäume wird als Maß für den Konkurrenzdruck angesehen. Dabei werden solche Bäume, die niedriger als der Zentralbaum sind, nicht betrachtet. Sie üben vermutlich keine Konkurrenz auf den Probebaum aus.

\section{$3.4 \quad H / D-W e r t$}

Zusammen mit der Kronenausbildung bildet der H/D-Wert (Schlankheitsgrad) einen wichtigen Indikator der Einzelbaumstabilität (KRAMER 1975). Er beschreibt das Verhältnis von Baumhöhe $(\mathrm{H})$ zu Stammdurchmesser (D) und vermittelt auf diese Weise einen Eindruck von der statischen Konstruktion des Baumes. Der H/D-Wert hat besonders für Nadelhölzer eine große Bedeutung. Hohe H/D-Werte (>80) weisen in Verbindung mit geringen Kronenprozenten auf eine erhöhte Instabilität des Baumes hin. Der H/D-Wert wird hauptsächlich durch die Bestandesbehandlung, insbesondere durch Bestandesbegründung und Durchforstungsstrategie beeinflußt (KRAMER et al 1988). Er kann als ein Ergebnis der Bestandesdichte aufgefaßt werden. Je dichter die Bäume zusammenstehen, desto mehr nimmt ihr Durchmesser bei annähernd gleichbleibender Höhe zu. Der H/D-Wert wird demzufolge höher.

\subsection{Kronenausdehnung}

Bei den Beständen in Holzerode und Kattenbühl wird die Kronenausdehnung gemessen. In Holzerode wird die Kronenfläche mittels Kronenspiegel und Abstand zum Stamm ermittelt (48 Radien). In Kattenbühl werden nach Entnahme des Probebaumes in den Hauptkoordinaten Nord, Ost, Süd und West die jeweiligen Kronenradien aufgenommen. Auf diese Weise ist eine Berechnung der Kronenfläche anhand des durchschnittlichen Kronendurchmessers möglich (Formel 4). 


$$
\begin{aligned}
& \mathrm{Kf}=\partial^{\star} r^{2} \\
& \mathrm{Kf}=\text { Kronenschirmfläche }\left(\mathrm{m}^{2}\right) \\
& \mathrm{R}=\text { durchschnittlicher Kronenradius }(\mathrm{m})
\end{aligned}
$$

\subsection{Probebaumentnahme}

Die Probebäume wurden nach längeren Regenperioden vorsichtig mittels eines Schleppers umgezogen (aus feuchtem Boden lassen sich die Wurzeln leichter herausziehen). Abgebrochene Wurzeln wurden nachgegraben und an den Bruchstellen am Wurzelsystem befestigt.

\subsection{Gewinnung und Aufnahmeverfahren der ober- und unteridischen Biomasse}

\section{Stamm}

Nach der Entnahme des Probebaumes wurden die Länge des Stammes, der Kronenansatz (ab drittem lebenden Ast) und die mittleren Durchmesser fortlaufender Sektionen, 0,25 m-0,75 m1,3 m-1,5 m-2,5 m-3,5 m usw., am liegenden Stamm gemessen. Mit Hilfe dieser abschnittsweisen Durchmesserdaten war die Volumenberechnung des Stammholzes mittels Formel 5 möglich.

$$
\begin{aligned}
& \begin{aligned}
V_{s t} & =\sum_{i}^{n}\left(G_{m} * l_{i}\right) \\
\mathrm{Gm} & =\text { Mittengrundfläche des zu berechnenden Körpers }(\mathrm{m}) \\
\mathrm{li} & =\text { Länge des zu berechnenden Körpers }(\mathrm{m})
\end{aligned} \\
& \text { Formel 5: Berechnung des Stammvolumens }\left(\mathrm{m}^{3}\right)
\end{aligned}
$$

\section{Krone}

Die Krone wurde im frischen Zustand vollständig gewogen. Anhand eines repräsentativen Probeastes aus der Kronenmitte konnte das Blatt-Ast (erste und zweite Ordnung)- Nebenästchen (dritte bis n-te Ordnung) - Verhältnis als Frisch- (FG) und Trockengewicht (TG) im Labor ermittelt werden. Anhand dieser Daten und der gemessenen Gesamtkronenmasse werden die Kronenbestandteile berechnet.

\section{Wurzel}

Nach den beschriebenen Aufnahmen wurde der Stamm in 0,25 m Höhe von dem Wurzelsystem abgetrennt. Die Wurzelsysteme wurden anfänglich mit einem Luftdruckgerät gesäubert. Dieser Aufwand, bei dem ein Kompressor mitsamt eines Ganzkörperschutzes benötigt wird, schien nach den 20 stärksten Wurzelsystemen und drei Tagen (zwei Mann) zu groß, so daß die restlichen Wurzeln per Hand mit Hilfe von Schaufel, Hacke und spitzen Eisenstangen gesäubert wurden. Die auf diese Weise gesäuberten 242 Wurzelsysteme waren zum größten Teil geringer dimensioniert und stammen zu $35 \%$ aus Sandbodenflächen. Durchschnittlich ist für die Reinigung ein Zeitaufwand von $45 \mathrm{~min} /$ Wurzel / Mann nötig.

Nach dem Säuberungsverfahren wurden die Wurzeln vermessen (Abschnitt 3.8.1). 


\subsection{Ermittlung der Grobwurzel- und Feinwurzelparameter}

\subsubsection{Wurzel-Meßverfahren}

In dieser Arbeit werden terminologisch alle Wurzeln, die einen größeren Durchmesser als $2 \mathrm{~mm}$ haben, als Grobwurzeln und die kleiner und gleich $2 \mathrm{~mm}$ (Durchmesser) als Feinwurzeln bezeichnet. Dimensionsverhältnisse werden durch die Wurzelquerschnittflächen charakterisiert.

Um qualitative und quantitative Aussagen zur Wurzelarchitektur machen zu können, muß jede Wurzel eines Wurzelsystems in bezug auf Verzweigung und Biomasse aufgenommen werden. Nach einem von NIELSEN entwickeltem Verfahren (ROOTARCH I 1995) werden die Wurzeln wie folgt vermessen.

Grundsätzlich ist eine Grobeinteilung des Wurzelsystems in horizontaler und vertikaler Richtung erforderlich. Der Horizontalwinkel gibt Aufschluß über die Himmelsrichtung, in die die Wurzel wächst, wobei $0^{\circ}$ Norden und $180^{\circ}$ Süden entsprechen (Abb. 6). Der Vertikalwinkel bestimmt die Tiefendurchwurzelung mit $0^{\circ}$ Flachwurzel bis hin zu $90^{\circ}$ Tiefenwurzel. Der Übergang von Flachwurzeln zu Tiefenwurzeln liegt bei $45^{\circ}$. Dabei wird eine Wurzel, deren Winkel kleiner gleich $45^{\circ}$ ist, als Flachwurzel bzw. eine, deren Winkel größer $45^{\circ}$ ist, als Tiefenwurzel eingeteilt. Darüber hinaus werden für jede Wurzel des Systems die maximale Tiefe und die geschätzte Anzahl der Feinwurzeln dokumentiert. Nach der Vermessung wird in einzelnen Beständen (Kattenbühl) das Gesamtwurzelsystem gewogen.

Zur genauen Vermessung der horizontalen Ebenen werden über die Gesamtwurzel fiktiv drei Zylinder in verschiedenen Radien gelegt (Tabelle 8). Diese sind abhängig vom Durchmesser der Probebäume. Je geringer die Probebaumdimensionen sind, desto enger müssen die Meßzylinder gelegt werden, da sonst zu wenig Wurzeln erfaßt werden. Tritt eine Wurzel durch den Zylinder der ersten, zweiten oder dritten Sektion, werden an der jeweiligen Durchtrittsstelle der Wurzelquerschnitt (zwei Messungen des Durchmessers) und die Durchtrittskoordinaten (Neigungswinkel) bestimmt. Bei der praktischen Durchführung der horizontalen Vermessung der Wurzeln werden markierte Zollstöcke über das Wurzelsystem gelegt und die Sektionen mit Kreide gekennzeichnet, so daß die Radien schnell erfaßbar sind.

Zur vertikalen Erfasssung werden in verschiedenen Tiefen Schnittebenen durch die Zylinder gelegt, in denen gleichsam die vorgenannten Bestimmungen erfolgen. Diese drei Schnittebenen werden an den Wurzeln eingezeichnet. Mit Erfassung der horizontalen und vertikalen Elemente erhält man eine räumliche Beschreibung der Wurzelgeometrie. Nach der Aufnahme einer Wurzel wird diese durch eine Wäscheklammer gekennzeichnet, so daß sie nicht doppelt vermessen werden kann.

Wurzeln, die in ihrem Ursprung horizontal verlaufen, in weiterer Entfernung von der Stammbasis ihre ursprüngliche Durchwurzelungsrichtung jedoch ändern, werden als Richtungswechsler bezeichnet. Sie werden in der vorliegenden Arbeit in die Kategorie eingestuft, in der sie sich in der ersten Sektion befanden. Die Information der Richtungsänderung ist dokumentiert, wird aber aus Gründen der Übersichtlichkeit nicht dargestellt. 
Für diese Arbeit ist für die um 60 jährigen Buchen ein Zeitaufwand von etwa sechs Stunden und für gleichaltrigen Fichte ca. vier Stunden zu kalkulieren. Für die jüngeren Fichten, Kiefern und Eichen (13-31 jährig) werden im Mittel zwei Stunden benötigt.

Tabelle 8: Größe der Radien und Schnittebenen der gemessenen Sektionen (Abstände von der Stammbasis (Abb. 6)

\begin{tabular}{|l|cc|cc|cc|}
\hline $\begin{array}{l}\text { Entfernung von Stammbasis } \\
\text { (cm) }\end{array}$ & \multicolumn{2}{|c|}{ Sektion 1 } & \multicolumn{2}{c|}{ Sektion 2 } & \multicolumn{2}{c|}{ Sektion 3 } \\
Wurzelebene & horizontal & vertikal & horizontal & vertikal & horizontal & vertikal \\
Holzerode (Fichte / Buche) & 30 & 40 & 60 & 60 & 100 & 90 \\
Krebeck (Fichte) & 30 & 40 & 60 & 60 & 100 & 90 \\
Knesebeck (Fichte / Kiefer) & 30 & 40 & 60 & 60 & 100 & 90 \\
Holzminden (Eiche) & 20 & 20 & 40 & 40 & 70 & 70 \\
Kattenbühl (Eiche) & 20 & 30 & 40 & 40 & 70 & 70 \\
\hline
\end{tabular}

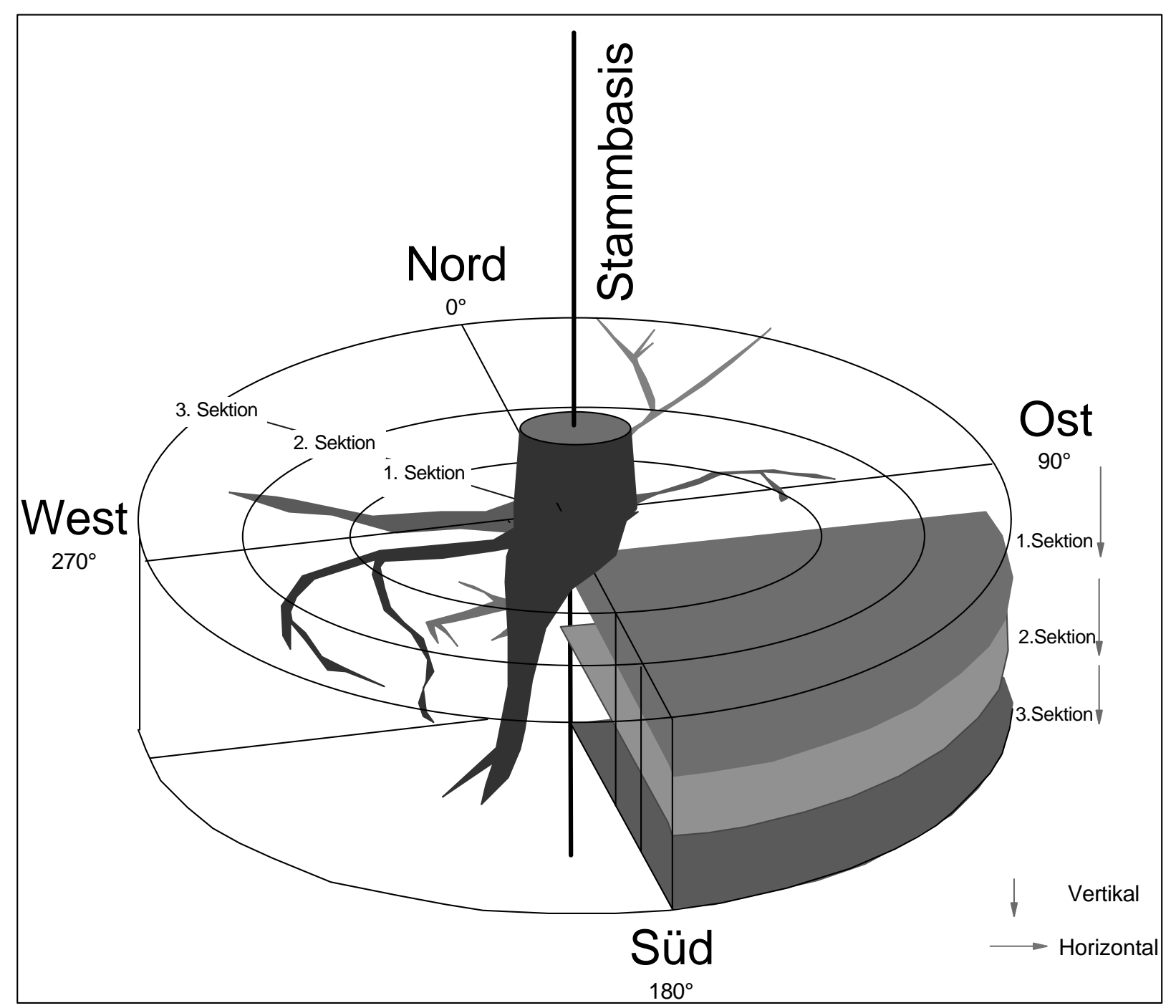

Abb. 6: Wurzelmeßschema 


\subsubsection{Feinwurzelermittlung}

Wie im vorherigen Abschnitt schon erwähnt, wird für jede Einzelwurzel eines Systems die Feinwurzelmenge geschätzt. Als Feinwurzeln gelten jene Wurzeln, die kleiner als $2 \mathrm{~mm}$ sind. Die Schätzung erfolgt nach Übung an 100 Einzelwurzeln, an denen nachträgliche Zählungen durch dieselben Personen durchgeführt wurden.

\subsubsection{Ermittlung der Einzelwurzeltiefe}

Bei der Aufnahme der Einzelwurzel wird die maximale Tiefe über die Sektionen hinaus dokumentiert. Die Vermessung erfolgt von der Bodenoberfläche, die durch die Markierung des Wurzelansatzes erkennbar ist, bis an die tiefste Stelle jeder Einzelwurzel.

\subsection{Zusammenfassung der erhobenen Parameter}

Folgende Baum- und Wurzeldaten stehen für die Analysen zur Verfügung (Tabelle 9).

Tabelle 9: Oberirdische Parameter

\begin{tabular}{|ll|}
\hline & Oberirdische Parameter \\
- Alter & - Kronengewicht (frisch, trocken) \\
- Höhe & - \% Anteile Blatt / Ast \\
- H/D-Wert & - Kronenansatz \\
- Heziale Stellung & - Kronenausdehnung (Kattenbühl, Holzerode) \\
- Überragungswinkel $(\alpha)$ & - Stammvolumen \\
\hline
\end{tabular}

Tabelle 10: Unterirdische Parameter

Wurzelparameter

- Wurzeldurchmesser der 1., 2., 3. Sekti- - Feinwurzelanzahl pro Einzelwurzel on (2 Messungen)

- Wurzelkoordinaten der 1., 2., und 3. - Gesamtwurzelgewicht (Kattenbühl) Sektion (horizontal und vertikal)

- Maximale Wurzeltiefe der Einzelwurzel

\subsection{Datenanalyse und Statistik}

\subsubsection{Datenauswertung}

Die Berechnung der Ergebnisse erfolgte unter zu Hilfenahme des Programms "TREEARCH" (NIELSEN 1995). Hiermit wurden die Querschnittsflächensummen jeder Sektion berechnet. Weiterführende Ergebnisse wurden mit den Programmen ExCEL, WinSTAT und STATISTICA erarbeitet. 
In einem nächsten Schritt wurde eine Plausibilitätskontrolle vorgenommen. Danach wurde geprüft, ob Normalverteilungen vorliegen. Der Kolmogorov-Smirnov-Test berechnet dafür die maximalen Distanzen zwischen den kontinuierlich verteilten Daten und der idealen kumulierten Häufigkeit. Eine Irrtumswahrscheinlichkeit von $p>5 \%$ wird als Signifikanzkriterium genutzt.

Liegt keine Normalverteilung vor, wurde entweder ein nichtparametrischer Mittelwertvergleichstest (Mann-Whitney U-Test) durchgeführt oder die Daten werden durch die BoxcoxFunktion transformiert. Hierbei wird die Schiefe der Verteilung reduziert. Diese wird definiert durch das Ersetzen von $x-\operatorname{Ln} x$ für $L=0$ - und $-X^{L}-1 / X$ für $L$ ungleich 0 . Mit Werten für $\mathrm{L}>1$ werden negative und mit $0<\mathrm{L}<1$ werden positive Schiefen behoben. Es kann vorkommen, daß darüber hinaus die Kurtosis behoben werden muß. Die Kurtosis charakterisiert die Abbweichung von der Normalverteilung. Dabei wird das $X$ folgendermaßen ersetzt: $\mathrm{x}-\operatorname{sign}\left[\left(\mathrm{X}-\mathrm{x}_{\mathrm{m}}+1\right)^{\mathrm{L}}\right]-1 / \mathrm{L}$ für $\mathrm{L}=0$ und $\mathrm{x}-\operatorname{sign}\left[\left(\mathrm{X}-\mathrm{x}_{\mathrm{m}}+1\right)^{\mathrm{L}}\right]$ für $\mathrm{L}$ ungleich 0 , wobei $\mathrm{L}=0,5$ gesetzt wird. Mit Werten für $\mathrm{L}>1$ wird eine negative und mit $0<\mathrm{L}<1$ wird eine positive Kurtosis behoben.

Mit der Varianzanalyse wurde geprüft, ob signifikante Unterschiede zwischen den Mittelwerten vorliegen (Tukey $\mathrm{p}=0,05$ ). Die homologen Gruppen werden in den Abbildungen durch einen Stern $(*)$ gekennzeichnet. Pfeile weisen darauf hin, welche Parameter sich signifikant von den gekennzeichneten Größen unterscheiden.

Für die Beurteilung statistischer Zusammenhänge zwischen den gemessen Daten werden Korrelations (Pearson)-und Regressionsanalysen durchgeführt (SACHS 1978). Durch die Methode der kleinsten Quadrate kann eine bestangepaßte Ausgleichsgerade- oder Kurve mit der dazu

gehörigen Funktion berechnet werden. Die Güte der Anpassung wird durch das Bestimmtheitsmaß $\left(R^{2}\right)$ bei einem Signifikanzniveau von $\mathrm{p}=0,05$ dokumentiert. Weiterhin stellt sich die Frage, inwiefern sich die Steigung der Regressionsgeraden signifikant voneinander unterscheidet. Es besteht die Möglichkeit, daß die Veränderung eines Faktors, z. B des Baumalters, die Ausgleichsgerade nur verschiebt. D.h., es ist keine Änderung der Steigungen vorhanden. Um dies zu prüfen, wird eine Kovarianzanalyse mit Test auf Parallelität durchgeführt. Dieser Test beruht auf dem Vergleich der Residual-Varianz/Kovarianz-Matrix innerhalb jeder Zelle $(\mathrm{p}=0,05)$.

Für die Beschreibung der normalverteilten Datensätze werden der arithmetische Mittelwert (x), die Standardabweichung (sx) und der Variationskoeffizient (v\%) berechnet. Die Standardabweichung wird in die jeweiligen Grafiken eingebunden (Pfeil).

\subsubsection{Weiterführende Berechnungen der Wurzelparameter}

\subsubsection{Erstellung der Häufigkeitsverteilungen der Wurzeln der ersten Sektion}

Für alle Bestände werden Häufigkeitsverteilungen der ersten Sektion in 10 mm-Klassen erstellt. Grundlage dieser Darstellung bilden alle gemessenen Wurzeldaten einer Versuchsfläche (Anhang II). Darüber hinaus wird, nach Baumdurchmesser differenziert, eine weitere Stratifizierung für die Fichten-, Buchen- und Kiefernwurzeln nach $50 \mathrm{~mm}$ und für die Eichen nach 
$20 \mathrm{~mm}$-Klassen vorgenommen. Diese werden für die verschiedenen Bestände als Kreisdiagramme der Vertikalen und Horizontalen dargestellt. Dabei sind die Kreisdiagramme der vertikalen Wurzeln aus Platzgründen kleiner dargestellt als die der horizontalen Wurzeln.

\subsubsection{Relation zwischen Horizontal- und Vertikalwurzeln}

Um eine Gleichmäßigkeit des Wurzelsystems in bis zu drei Kubikmetern Boden (abhängig von Sektionengröße bzw. Baumdurchmesser) charakterisieren zu können, wird die horizontale Wurzelanzahl zu der vertikalen ins Verhältnis gesetzt. Gleiches wird für die Wurzelquerschnittfläche vorgenommen. In Abb. 7 werden exemplarisch diese Relationen dargestellt. Eine Voraussetzung für eine für die Stabilität des Baumes optimale Ausbildung eines Wurzelsystems ist die gleichmäßige Verteilung der Wurzelbiomasse im Boden (NIELSEN 1990). Darüber hinaus kann das Verhältnis der horizontalen und vertikalen Wurzelquerschnittflächen in definierten Schichtebenen als ein Maß der "mechanischen und physiologischen Verankerung des Wurzelsystems betrachtet werden" (GRUBER 1992).

\begin{tabular}{|c|c|c|c|c|c|c|c|c|}
\hline & Ausg & ewogene & Wurzelsy & tem & Unau & yewogen & es Wurzelsy & stem \\
\hline & Anzal & I $(N)$ & WQF & $\left(\mathrm{cm}^{2}\right)$ & Anza & $(N)$ & $W Q F$ & $\left(\mathrm{~cm}^{2}\right)$ \\
\hline & horizonta & vertika & horizonta & vertikal & horizonta & vertika & horizonta & vertikal \\
\hline Sektion 1 & 1 & 1 & 5 & 5 & 1 & 1 & 5 & 5 \\
\hline Sektion 2 & 1 & 1 & 3 & 3 & 2 & 1 & 8 & 3 \\
\hline Sektion 3 & 1 & 1 & 2 & 2 & 4 & 2 & 4 & 2 \\
\hline Sektion 1 & 1: & & 1 & & 1 & & & $: 1$ \\
\hline Sektion 2 & 1: & & 1 & & 2 & & & \\
\hline Sektion 3 & 1: & & 1 & & 2 & & & $: 1$ \\
\hline
\end{tabular}

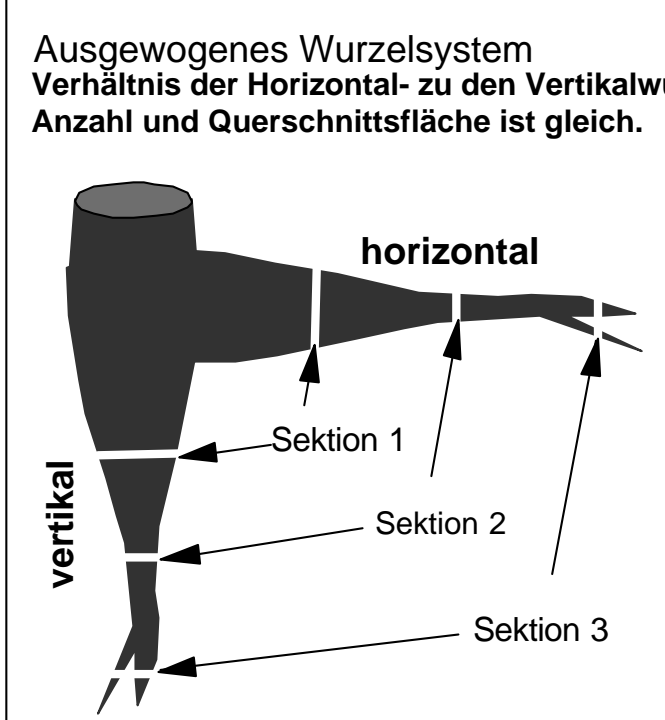

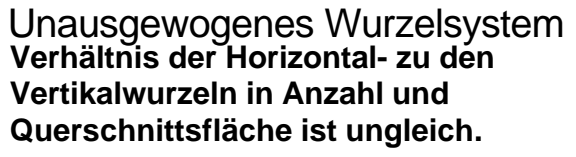

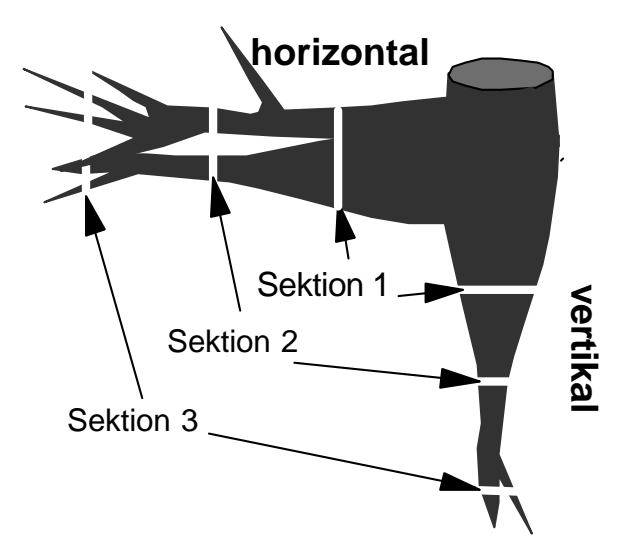

Abb. 7: Erklärende Darstellung der Verhältnisbetrachtung der Horizontal- zu den Vertikalwurzeln in Anzahl (N) und Wurzelquerschnittfläche (WQF $\mathrm{cm}^{2}$ ) in den drei Sektionen. Ausgewogenes Wurzelsystem wird durch eine Relation von 1:1, unausgewogenes durch z.B. 5:1 gekennzeichnet. 
Ein Wurzelanzahlverhältnis von 1: 1 in z.B. $30 \mathrm{~cm}$ Radius von der Stammbasis (erste Sektion) läßt auf eine gleichmäßige Wurzelausbildung schließen, da ebensoviele Horizontal- wie Vertikalwurzeln vorhanden sind. Ein Verhältnis von z.B. $5: 1$ besagt, daß das Wurzelsystem in einer bestimmten Sektion fünfmal mehr Horizontalwurzeln als Vertikalwurzeln besitzt.

Jedoch ist das oben beispielhaft dargestellte Verhältnis von 1:1 nicht allein aussagekräftig, da die vertikalen Wurzeln durchaus dicker sein können als die horizontalen und umgekehrt. Die Stärke der Wurzeln wird durch die Summe der Einzelwurzelquerschnittflächen des gesamten Wurzelsystems erklärt und ebenfalls in Relation gesetzt. In gemeinsamer Betrachtung der Wurzelanzahl und der Querschnittsflächen kann so die Wurzelsystemausformung und damit die Verteilung der Biomasse beschrieben werden. Z.B. liegt das Anzahlverhältnis bei 1: 1, das Querschnittsflächenverhältnis aber bei 10:1. Dieses Wurzelsystem besitzt demnach bei gleich vielen Horizontal- wie Vertikalwurzeln 10 mal dünnere Wurzeln in der Vertikalen als in der Horizontalen. Durch die relative Betrachtung ist es möglich, Baumarten und Altersstufen untereinander zu vergleichen. Das Flachwurzelsystem, das für die Fichte als typisch beschrieben wird, kann ebenso wie ein Herzwurzelsystem der Buche charakterisiert werden.

Zeigt das Wurzelsystem eines Baumes in Anzahl und Wurzelquerschnittfläche ein Verhältnis der Horizontal- zu Vertikalwurzeln von 1:1, so ist von der Form eines Zylinders auszugehen. Die Ausformung dieses Kreiskegels stellt nach SCHMID-HAAS und BACHHOFEN (1991) ein gutes Maß für die Stabilität des Baumes dar.

Beispielhaft werden für einen Eichenbestand neben den relativen Zahlen auch die Absolutwerte grafisch dargestellt. Für die anderen Bestände werden Tabellen erstellt, die die prozentualen Anzahl- und Querschnittsflächenanteile erklären.

\subsubsection{Berechnung des Wurzelvolumens}

Zur Berechnung des Wurzelvolumens wird die "Newton'sche Formel" angewendet (Formel 6). Mit der Aufnahme der drei in der Horizontalen und Vertikalen gemessenen Sektionen ist die Möglichkeit der Bildung einer Ausgleichsfunktion (log) gegeben. Als Grundwert der Ausgleichsfunktion wird die summierte Wurzelquerschnittfläche aller Einzelwurzeln der horizontalen oder vertikalen drei Sektionen betrachtet. Die Berechnung der Ausgleichsfunktion wird für jedes einzelne Wurzelsystem durchgeführt. Diese Einzelbaumwerte sind die Grundlage der Bestandesdaten. Lassen sich die drei Werte nicht durch eine ausreichend angepaßte Funktion erklären $(\mathrm{R}<0,5)$, fällt der Baum aus der Bestimmung der Bestandeswerte heraus.

Die für die Newton'sche Formel benötigten Grundflächen der stammnahen Wurzelbereiche lassen sich durch Extrapolation des oben beschriebenen Zusammenhangs zwischen Wurzelquerschnittfläche und Entfernung vom Wurzelzentrum herleiten. Die mittlere und die untere Grundfläche konnten anhand der Funktion geschätzt werden. Dies ist für die Anwendung der Newton'sche Formel (Formel 6) notwendig, bei der die Grundflächen des unteren, mittleren und oberen Wurzelastabschnittes benötigt werden. 


\begin{tabular}{|l|}
$\mathbf{v}_{\mathbf{w}}=\mathrm{I}^{\star}(\mathbf{g u}+\mathbf{4 g m + g o}) / \mathbf{6}$ \\
$\mathrm{I} \quad=$ Länge der Wurzel $(\mathrm{dm})$ \\
$\mathrm{gu}=$ untere Grundfläche $\left(\mathrm{dm}^{2}\right)$ \\
$\mathrm{gm}=$ mittlere Grundfläche $\left(\mathrm{dm}^{2}\right)$ \\
go $=$ obere Grundfläche $\left(\mathrm{dm}^{2}\right)$
\end{tabular}

Formel 6: Newton'sche Formel (KRAMER und AKCA 1987) zur Berechnung des Wurzelvolumens $\left(d m^{3}\right)$

In Abhängigkeit des Baumalters wird für den Wert der unteren Grundfläche die Entfernung in $10 \mathrm{~cm}$ bzw. $20 \mathrm{~cm}$ von der Stammbasis gewählt, da in den meisten Fällen erst dort eine eindeutige Wurzelastausprägung $\mathrm{zu}$ beobachten ist. Demzufolge wird das Wurzelvolumen erst ab diesem Bereich berechnet, so daß nur das Wurzelastvolumen wiedergegeben wird.

Für die Werte der mittleren Grundfläche werden abhängig von der Dimension des Wurzelsystems die Entfernungen 40 bis $60 \mathrm{~cm}$ gewählt und für die oberen 70 bis $100 \mathrm{~cm}$ von der Stammbasis.

\subsubsection{Oberfläche einer Einzelwurzel}

\section{Berechnung der Wurzeloberfläche}

Für die Schätzung der Wurzeloberfläche wird die Formel zur Berechnung der Oberfläche eines Kegelstumpfes (Abb. 8) verwendet. Als Eingangsgrößen werden der Radius des oberen und unteren Wurzelabschnittes und die Mantellinie benötigt (Formel 7). Beide Größen sind nicht verfügbar und müssen kalkuliert werden. Bei der Anwendung dieser Formel werden der untere und der obere Querschnitt der Wurzel in die Berechnung der Gesamtoberfläche mit einbezogen.

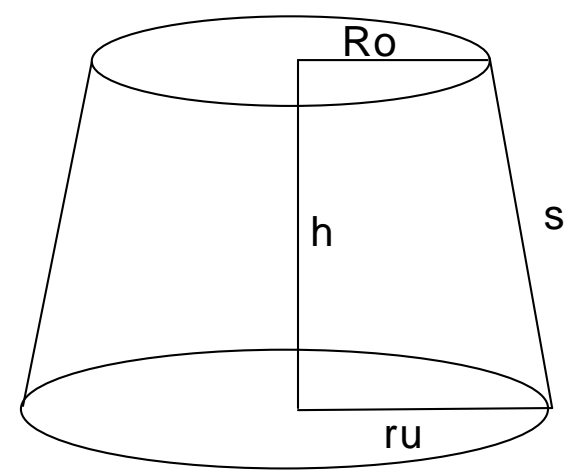

Abb. 8: Kegelstumpf

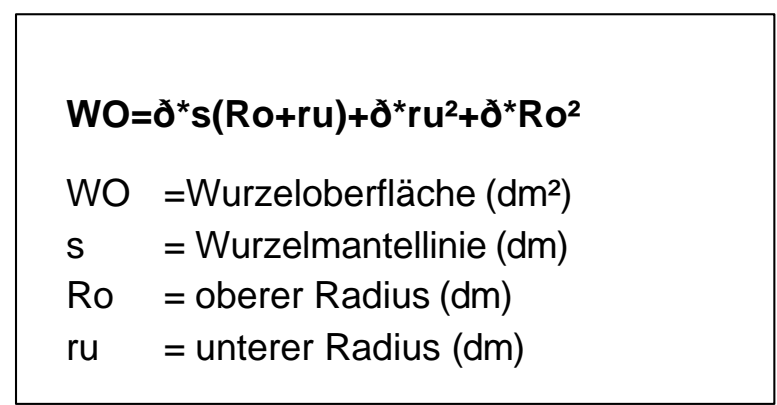

Formel 7: Wurzeloberfläche

\section{Ermittlung der Radien}

Anhand des durchschnittlichen Wurzeldurchmessers in den drei gemessenen Sektionen wird für eine mittlere horizontale und vertikale Wurzel eine logarithmische Ausgleichsfunktion arstellt (Tabelle 11). Die Abnahme der Wurzeldurchmesser der Einzelwurzeln unterscheidet sich mit zunehmender Entfernung vom Wurzelzentrum nicht signifikant. Mit Hilfe der Funktionen können die Wurzeldurchmesser in einer beliebigen Entfernung zum Wurzelstock geschätzt werden. Die Funktion lautet $\mathrm{y}=\mathrm{b}^{*} \operatorname{Ln}(\mathrm{x})+\mathrm{a}$. Dabei beschreibt y den Durchmesser und $\mathrm{x}$ die Länge des Wurzelabschnitts. 
Tabelle 11: Ausgleichsfunktionen zur Bestimmung der Wurzeldurchmesser in einer beliebigen Entfernung vom Wurzelstock; $x=$ Entfernung vom Wurzelstock; $y=$ Wurzeldurchmesser

\begin{tabular}{|c|c|c|c|c|c|}
\hline & Bestand & Horizontal & $\mathrm{R}^{2}$ & Vertikal & $\mathrm{R}^{2}$ \\
\hline \multirow{4}{*}{$\begin{array}{l}\frac{0}{8} \\
\frac{0}{0} \\
\text { № } \\
\text { 온 }\end{array}$} & Buche & $y=-17,691 \operatorname{Ln}(x)+83,679$ & 0,99 & $y=-20,446 \operatorname{Ln}(x)+91,489$ & 0,94 \\
\hline & $\mathrm{Bu} / \mathrm{Fi}$ & $y=-18,388 \operatorname{Ln}(x)+87,106$ & 0,99 & $y=-22,442 \operatorname{Ln}(x)+98,67$ & 0,89 \\
\hline & Fichte & $y=-38,011 \operatorname{Ln}(x)+178,28$ & 0,96 & $y=-47,756 \operatorname{Ln}(x)+209,13$ & 0,88 \\
\hline & $\mathrm{Fi} / \mathrm{Bu}$ & $y=-39,388 \operatorname{Ln}(x)+200,45$ & 0,95 & $y=-54,156 \operatorname{Ln}(x)+244,3$ & 0,90 \\
\hline \multirow{3}{*}{ 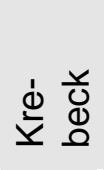 } & Fichte 21 & $y=-11,514 \operatorname{Ln}(x)+54,54$ & 0,96 & $y=-14,36 \operatorname{Ln}(x)+62,986$ & 0,87 \\
\hline & Fichte 30 & $y=-19,997 \operatorname{Ln}(x)+94,885$ & 0,95 & $y=-25,197 \operatorname{Ln}(x)+110,52$ & 0,90 \\
\hline & Fichte 41 & $y=-26,505 \operatorname{Ln}(x)+129,54$ & 0,99 & $y=-35,364 \operatorname{Ln}(x)+156,34$ & 0,90 \\
\hline \multirow{6}{*}{ 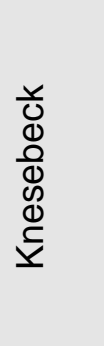 } & Fichte 50 & $y=-24,677 \operatorname{Ln}(x)+126,24$ & 0,98 & $y=-37,813 \operatorname{Ln}(x)+167,18$ & 0,90 \\
\hline & Fichte 31 & $y=-14,394 \operatorname{Ln}(x)+71,455$ & 0,98 & $y=-20,375 \operatorname{Ln}(x)+90,047$ & 0,90 \\
\hline & Fichte 20 & $y=-7,2344 \operatorname{Ln}(x)+33,603$ & 0,99 & $y=-8,8184 \operatorname{Ln}(x)+38,342$ & 0,86 \\
\hline & Kiefer 47 & $y=-20,473 \operatorname{Ln}(x)+97,317$ & 0,98 & $y=-25,16 \operatorname{Ln}(x)+111,19$ & 0,90 \\
\hline & Kiefer 33 & $y=-11,699 \operatorname{Ln}(x)+54,437$ & 0,97 & $y=-13,941 \operatorname{Ln}(x)+60,952$ & 0,89 \\
\hline & Kiefer 20 & $y=-4,9472 \operatorname{Ln}(x)+22,723$ & 0,98 & $y=-5,7708 \operatorname{Ln}(x)+25,17$ & 0,88 \\
\hline \multirow{3}{*}{ 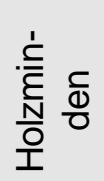 } & Pflanzung & $y=-6,1397 \operatorname{Ln}(x)+29,521$ & 0,99 & $y=-8,1058 \operatorname{Ln}(x)+3$ & 0,88 \\
\hline & Saat & $y=-6,9303 \operatorname{Ln}(x)+33,657$ & 0,96 & $y=-9,5306 \operatorname{Ln}(x)+41,763$ & 0,90 \\
\hline & Verpflanzung & $y=-4,9472 \operatorname{Ln}(x)+22,723$ & 0,98 & $y=-8,5332 \operatorname{Ln}(x)+37,175$ & 0,89 \\
\hline \multirow{3}{*}{ 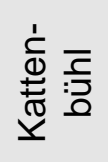 } & Pflanzung & $y=-0,7724 \operatorname{Ln}(x)+7,5694$ & 0,91 & $y=-7,5685 \operatorname{Ln}(x)+31,535$ & 0,89 \\
\hline & Saat I & $y=-2,703 \operatorname{Ln}(x)+13,67$ & 0,98 & $y=-10,534 \operatorname{Ln}(x)+44,222$ & 0,96 \\
\hline & Saat II & $y=-0,9346 \operatorname{Ln}(x)+6,7455$ & 0,73 & $y=-7,1719 \operatorname{Ln}(x)+29,957$ & 0,94 \\
\hline
\end{tabular}

\section{Berechnung der Wurzelmantellinie}

Weiterhin wird für die Formel der Wurzeloberfläche (Formel 7) die Wurzelmantellinie benötigt (Formel 8). Diese kann mit der Wurzellänge berechnet werden, die durch die Funktionen der Tabelle 11 kalkuliert wird. Die durchschnittlichen Wurzellängen liegen bei allen Beständen horizontal zwischen $80 \mathrm{~cm}$ und $150 \mathrm{~cm}$ und vertikal zwischen $65 \mathrm{~cm}$ und $80 \mathrm{~cm}$ (Tabelle 12).

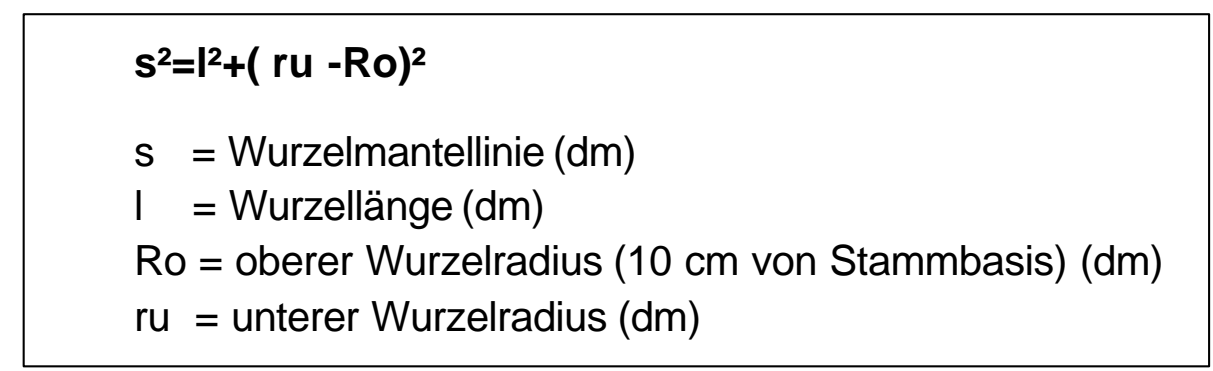

Formel 8: Berechnung der Wurzelmantellinie 
Tabelle 12: Nach den Ausgleichsfunktionen (Tabelle 11) berechnete Wurzellängen (mittlere Einzelwurzeln der untersuchten Bestände)

\begin{tabular}{|c|c|c|c|c|c|c|c|}
\hline & & Wurzellän & e $(\mathrm{cm})$ & & & Wurzellän & e $(\mathrm{cm})$ \\
\hline & Bestand & horizontal & vertikal & & Bestand & horizontal & vertikal \\
\hline 0 & Buche & 100 & 75 & y & & & \\
\hline 인 & Buche / Fichte & 100 & 70 & О & Kiefer 47 & 105 & 70 \\
\hline$\frac{\mathrm{N}}{\mathrm{O}}$ & Fichte & 95 & 65 & $\Phi$ & Kiefer 33 & 90 & 65 \\
\hline & Fichte / Buche & 150 & 80 & $\bar{x}$ & Kiefer 20 & 80 & 65 \\
\hline & Fichte 41 & 120 & 70 & & Pflanzung & 100 & 65 \\
\hline $\begin{array}{ll}0 & 0 \\
\grave{1} & 0 \\
0\end{array}$ & Fichte 30 & 105 & 70 & & Saat & 110 & 65 \\
\hline & Fichte 21 & 100 & 70 & & Verpflanzung & 80 & 65 \\
\hline & Fichte 50 & 155 & 70 & & Pflanzung & 90 & 50 \\
\hline $\begin{array}{ll}0 \\
\Phi\end{array}$ & Fichte 31 & 130 & 70 & $\underset{\sigma}{\stackrel{\Phi}{ \pm}: ~}$ & Saat I & 90 & 55 \\
\hline & Fichte 20 & 90 & 65 & & Saat II & 90 & 55 \\
\hline
\end{tabular}

Nachdem die Mantellinie der Wurzel mit Hilfe von Formel 8 bestimmt wurde, kann die Wurzeloberfläche (Formel 7) berechnet werden.

Berechnet wird hier allein eine mittlere Wurzel des Bestandes, deren Wert abschließend mit der durchschnittlichen Wurzelanzahl multipliziert wird. Dieser Wert beschreibt die Wurzeloberfläche der Grobwurzeln eines Wurzelsystems. Betont werden muß, daß es sich bei diesen kalkulierten Daten nur um Schätzwerte handelt, da die Berechnung nur modellhaft erfolgen konnte.

\subsection{Erstellen von Flußdiagrammen zur Beschreibung der oberirdischen- und unterirdischen Parameter}

Nach Berechnung der ober- und unterirdischen Daten werden diese ins Verhältnis gesetzt und geprüft, ob Abhängigkeiten zwischen den Parametern bestehen. Die Ergebnisse werden in Flußdiagrammen dargestellt (Abb. 9). Dabei werden folgende Größen berücksichtigt:

- $\quad \mathrm{BHD}(\mathrm{cm})$

- Höhe (m)

- Kronengewicht (frisch) $(\mathrm{kg})$
- Blattgewicht $(\mathrm{kg})$

- in Kattenbühl Wurzelgewicht $(\mathrm{kg})$

In einem Fall wird, da das Blattgewicht nicht vorhanden ist, der Kronenansatz als Bezugseinheit gewählt.

Es werden sechs Grafiken so wiedergegeben, daß Stammvolumen $\left(\mathrm{m}^{3}\right)$ und Wurzelvolumen $\left(\mathrm{dm}^{3}\right)$ die $\mathrm{x}$-Achse darstellen und die anderen Parameter die y-Achse. Abb. 9 zeigt beispielhaft die Aufteilung einer solchen Seite. Es ist so möglich, mit diesen Grafiken anhand eines festgelegten Baumdurchmessers die oben genannten Größen abzulesen. Genauer ist das Ergebnis jedoch durch die Berechnung der verschiedenen Werte, die nachfolgend beschrieben wird. Mit dem BHD (erste y-Achse), als leicht zu ermittelnde Eingangsgröße, wird durch eine lineare Funktion des Kästchens A das Stammvolumen ermittelt. Mit diesem Wert können darauffolgend die Höhe (B; erste y-Achse) und das Kronengewicht $(\mathbf{C})$ berechnet werden. In Ab- 
folge der Pfeile werden die übrigen Parameter kalkuliert. Auch möglich ist es, mit anderen Werten in die Berechnung einzusteigen. In dieser Arbeit wird jedoch immer die vorher beschriebene Reihenfolge eingehalten.

Die in der Grafik dargestellten Kronenparameter werden im frischen Zustand dargestellt. In Tabelle 13 sind die prozentualen Feuchtegehalte der Kronen wiedergegeben, so daß das Trockengewicht berechnet werden kann.

Tabelle 13: Prozentualer Wassergehalt der Krone

\begin{tabular}{|llll|}
\hline Bestand & $\%$ Wassergehalt & Bestand & \% Wassergehalt \\
Holzerode & 51,1 & Holzminden & 45,7 \\
Krebeck & 56,4 & Kattenbühl & 58,1 \\
Knesebeck & 43,2 & & \\
\hline
\end{tabular}

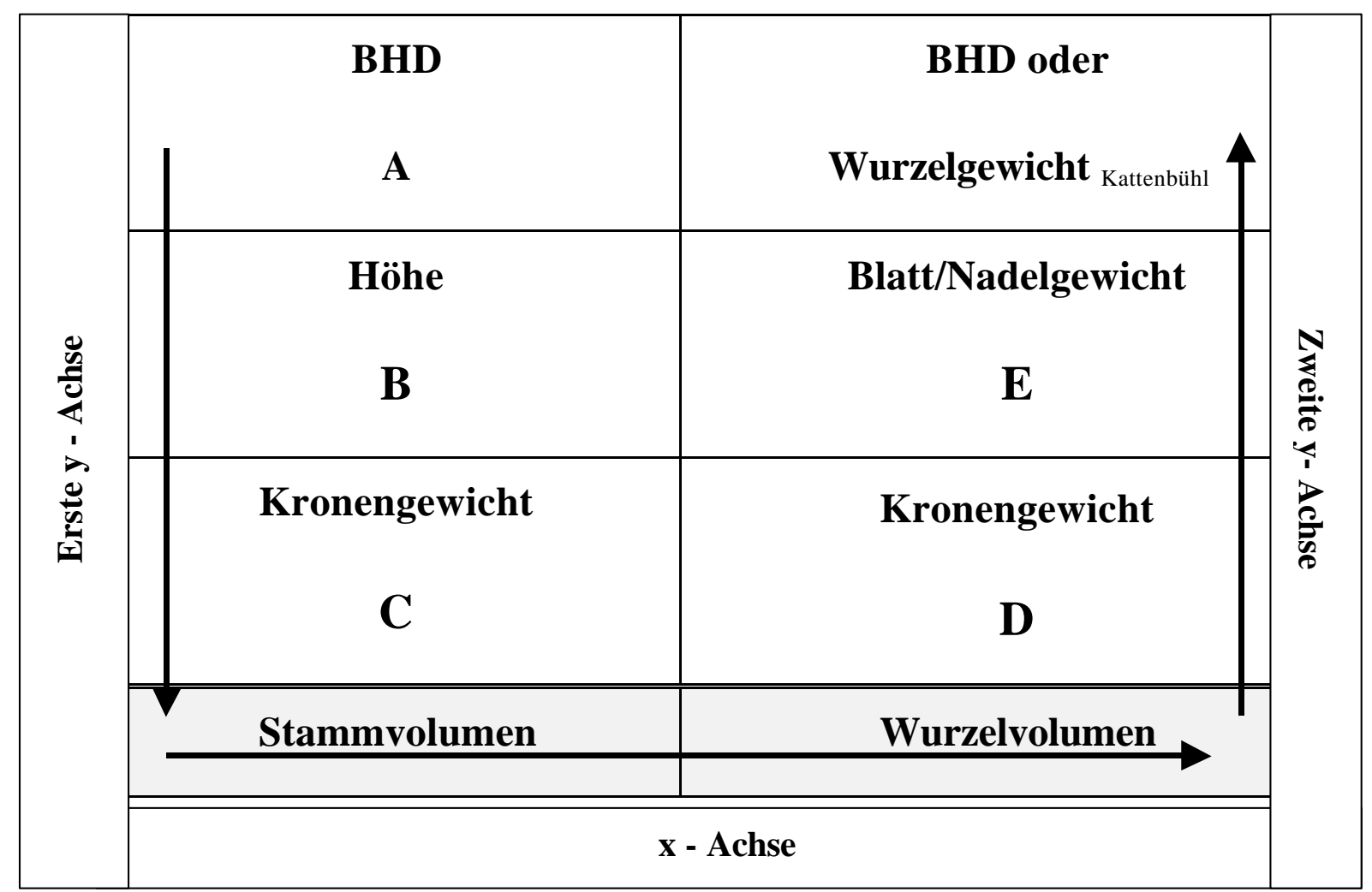

Abb. 9: Schema eines Flußdiagrammes

\subsection{Formelsammlung}

Die zur Berechnung der Wurzelparameter genutzten Formeln werden zusammenfassend im Anhang III dargestellt. 


\section{Ergebnisse}

\subsection{Oberirdische Parameter}

\subsubsection{Konkurrenzbedingungen}

\subsubsection{HEGYI Index}

\section{Holzerode}

In Holzerode werden der Abstand und der BHD der Nachbarbäume (bis zu neun) aufgenommen. Anhand dieser Daten wird der HEGYI-Index berechnet und in Tabelle 14 für die einzelnen Kraft'schen Klassen dargestellt. Zur weiteren Information sind die mittleren Abstände der drei nächsten Nachbarn und die Anzahl der nach BIGING und DOBBERTIN (1992) errechneten Konkurrenten aufgeführt. Insgesamt ist für alle Kraft'sche Klassen zu beobachten, daß die Konkurrenz, beschrieben durch den HEGYI-Index, bei den Buchenbeständen signifikant höher ist als bei den Fichtenbeständen. Ferner steigt der HEGYI-Index mit Abfall der sozialen Stellung an.

Der mittlere Baumabstand ist bei den Fichten des Mischbestandes am weitesten, am engsten stehen die Bäume im Buchenmischbestand.

Tabelle 14: Konkurrenzbeschreibende Daten; Holzerode, Buche und Fichte 55-65 jährig; (HEGYI-Index, Anzahl der Konkurrenten, Baumabstand der nächsten drei Nachbarn zum Probebaum); der Stern * beschreibt die signifikanten Unterschiede (fTest) des HEGYI-Index $(p=0,05)$ in Richtung der Pfeile

\begin{tabular}{|c|c|c|c|c|c|}
\hline & HEGYI & Buche & $\begin{array}{c}\text { Buche / Fichte } \\
*\end{array}$ & $\stackrel{\text { Fichte }}{\longrightarrow}$ & $\stackrel{\text { Fichte/ Buche }}{\longrightarrow}$ \\
\hline \multirow[t]{3}{*}{ Kraft 1} & HEGYI & & 2,02 & & 1,07 \\
\hline & Anzahl Konkurrenten & & 7,00 & & 5,50 \\
\hline & Nachbar 1 & & 2,55 & & 2,22 \\
\hline \multirow{2}{*}{$\begin{array}{c}\text { Abstände } \\
(\mathrm{m})\end{array}$} & Nachbar 2 & & 2,65 & & 2,31 \\
\hline & Nachbar 3 & & 2,80 & & 2,60 \\
\hline \multirow[t]{3}{*}{ Kraft 2} & HEGYI & 1,88 & 2,31 & 1,52 & 1,17 \\
\hline & Anzahl Konkurrenten & 5,75 & 7,00 & 5,50 & 6,75 \\
\hline & Nachbar 1 & 2,20 & 1,59 & 2,54 & 3,22 \\
\hline \multirow{2}{*}{$\begin{array}{c}\text { Abstände } \\
(\mathrm{m})\end{array}$} & Nachbar 2 & 3,45 & 2,03 & 3,27 & 4,20 \\
\hline & Nachbar 3 & 4,43 & 3,04 & 3,81 & 4,60 \\
\hline \multirow[t]{3}{*}{ Kraft 3} & HEGYI & 2,02 & 2,53 & & 1,50 \\
\hline & Anzahl Konkurrenten & 4,67 & 6,00 & & 5,33 \\
\hline & Nachbar 1 & 1,97 & 1,94 & & 2,71 \\
\hline \multirow{2}{*}{$\begin{array}{l}\text { Abstände } \\
\text { (m) }\end{array}$} & Nachbar 2 & 3,07 & 3,10 & & 3,45 \\
\hline & Nachbar 3 & 3,90 & 3,81 & & 3,81 \\
\hline
\end{tabular}




\begin{tabular}{|c|c|c|c|c|c|}
\hline & HEGYI & Buche & $\begin{array}{c}\text { Buche / Fichte } \\
*\end{array}$ & $\stackrel{\text { Fichte }}{\longrightarrow}$ & $\stackrel{\text { Fichte/ Buche }}{\longrightarrow}$ \\
\hline \multirow[t]{3}{*}{ Kraft 4} & HEGYI & & & & 2,05 \\
\hline & Anzahl Konkurrenten & & & & 4,00 \\
\hline & Nachbar 1 & & & & 1,22 \\
\hline Abstände & Nachbar 2 & & & & 2,80 \\
\hline (m) & Nachbar 3 & & & & 3,38 \\
\hline
\end{tabular}

\section{Krebeck}

Für die folgenden Bestände kann nur ein vereinfachter HEGYI-Index berechnet werden, da lediglich die Durchmesser und die Abstände zwischen Probebaum und den drei nächsten Nachbarn ermittelt wurden.

Der vereinfachte HEGYI-Index steigt mit Abnahme der sozialen Stellung an (Tabelle 15). Die Werte des jüngsten Bestandes weisen auf einen signifikant stärkeren Konkurrenzdruck hin, welches auf die hohe Bestandesdichte zurückzuführen ist. Die Werte der beiden älteren Flächen differieren nur wenig. Im Mittel der Kraft'schen Klassen zeigt der ältere Bestand einen um 0,23 geringeren HEGYI-Index als die 30 jährige Fichtenfläche. Dies resultiert aus niedrigeren Werten der Kraft'schen Klassen 2 und 4. Die Abstände des nächsten Nachbarn (Nachbar 1) steigen vom jungen zum alten Bestand an und fallen von den herrschenden $\mathrm{zu}$ den beherrschten Bäumen ab.

Tabelle 15: Konkurrenzbeschreibende Daten; Krebeck, Fichte 21-41 jährig; (HEGYI-Index, Baumabstand der nächsten drei Nachbarn zum Probebaum); der Stern * beschreibt die signifikanten Unterschiede (f-Test) des HEGYI-Index $(p=0,05)$ in Richtung der Pfeile

\begin{tabular}{|c|c|c|c|c|}
\hline & HEGYI & $\begin{array}{c}\text { Fichte } 21 \\
*\end{array}$ & Fichte 30 & $\stackrel{\text { Fichte }}{\longrightarrow}$ \\
\hline \multirow[t]{2}{*}{ Kraft 1} & HEGYI & 1,30 & 0,79 & 0,90 \\
\hline & Nachbar 1 & 1,95 & 2,15 & 2,34 \\
\hline \multirow[t]{2}{*}{ Abstände (m) } & Nachbar 2 & 2,20 & 2,45 & 2,52 \\
\hline & Nachbar 3 & 2,25 & 2,60 & 2,56 \\
\hline \multirow[t]{2}{*}{ Kraft 2} & HEGYI & 1,99 & 1,29 & 1,24 \\
\hline & Nachbar 1 & 1,37 & 1,60 & 1,82 \\
\hline \multirow[t]{2}{*}{ Abstände (m) } & Nachbar 2 & 1,40 & 1,95 & 1,82 \\
\hline & Nachbar 3 & 2,13 & 2,20 & 2,22 \\
\hline \multirow[t]{2}{*}{ Kraft 3} & HEGYI & 2,61 & 2,21 & 2,23 \\
\hline & Nachbar 1 & 1,40 & 1,63 & 1,65 \\
\hline \multirow[t]{2}{*}{ Abstände (m) } & Nachbar 2 & 1,90 & 1,83 & 1,95 \\
\hline & Nachbar 3 & 2,15 & 2,05 & 2,45 \\
\hline \multirow[t]{2}{*}{ Kraft 4} & HEGYI & 3,18 & 2,63 & 2,44 \\
\hline & Nachbar 1 & 1,53 & 1,25 & 1,80 \\
\hline \multirow[t]{2}{*}{ Abstände (m) } & Nachbar 2 & 1,95 & 2,27 & 1,90 \\
\hline & Nachbar 3 & 2,10 & 2,47 & 2,93 \\
\hline
\end{tabular}




\section{Knesebeck}

Auch hier nimmt der HEGYI-Index mit Abnahme der sozialen Stellung und des Alters zu, wobei ein deutlicher Unterschied zwischen Fichte und Kiefer zu beobachten ist (Tabelle 16). Die Kiefern stehen unter einem größeren Konkurrenzdruck als die Fichten. Weiterhin steigt die Konkurrenz mit Verschlechterung der sozialen Stellung stärker an, als das bei der Fichte der Fall ist. Besonders fällt hierbei der 33 jährige Kiefernbestand auf, bei dem der HEGYI-Index signifikant höher ist als bei der älteren Fläche.

Tabelle 16: Konkurrenzbeschreibende Daten; Knesebeck, Fichte und Kiefer, 31-50 jährig; (HEGYI-Index, Baumabstand der nächsten drei Nachbarn zum Probebaum); der Stern * beschreibt die signifikanten Unterschiede (f-Test) des HEGYI-Index $(p=0,05)$ in Richtung der Pfeile

\begin{tabular}{|c|c|c|c|c|c|}
\hline & Hegyi & Fichte 31 & Fichte 50 & $\begin{array}{l}\text { Kiefer } 33 \\
-*\end{array}$ & $\stackrel{\text { Kiefer } 47}{\longrightarrow}$ \\
\hline \multirow[t]{2}{*}{ Kraft 1} & HEGYI & 1,00 & 0,60 & 1,04 & 0,94 \\
\hline & Nachbar 1 & 2,00 & 2,66 & 1,44 & 2,14 \\
\hline \multirow[t]{2}{*}{ Abstände $(\mathrm{m})$} & Nachbar 2 & 2,26 & 2,81 & 1,73 & 2,87 \\
\hline & Nachbar 3 & 2,58 & 3,23 & 2,02 & 3,40 \\
\hline \multirow[t]{2}{*}{ Kraft 2} & HEGYI & 1,41 & 0,94 & 2,25 & 1,12 \\
\hline & Nachbar 1 & 2,20 & 2,58 & 1,30 & 2,38 \\
\hline \multirow[t]{2}{*}{ Abstände (m) } & Nachbar 2 & 2,47 & 2,82 & 1,53 & 2,81 \\
\hline & Nachbar 3 & 2,98 & 3,13 & 2,01 & 3,03 \\
\hline \multirow[t]{2}{*}{ Kraft 3} & HEGYI & 2,03 & 1,38 & 4,57 & 1,36 \\
\hline & Nachbar 1 & 1,86 & 2,18 & 0,65 & 2,65 \\
\hline \multirow[t]{2}{*}{ Abstände (m) } & Nachbar 2 & 1,89 & 2,70 & 1,53 & 2,82 \\
\hline & Nachbar 3 & 1,98 & 2,72 & 1,81 & 3,03 \\
\hline \multirow[t]{2}{*}{ Kraft 4} & HEGYI & 2,50 & 1,62 & 5,53 & 2,01 \\
\hline & Nachbar 1 & 1,92 & 1,05 & 0,69 & 2,17 \\
\hline \multirow[t]{2}{*}{ Abstände (m) } & Nachbar 2 & 2,20 & 2,30 & 1,52 & 2,87 \\
\hline & Nachbar 3 & 2,62 & 2,90 & 1,56 & 2,92 \\
\hline
\end{tabular}




\section{Holzminden}

Die herrschenden Saateichen besitzen mit dem HEGYI-Index von 2,19 die geringste Konkurrenz (Tabelle 17). Die Unterschiede sind jedoch nicht signifikant. Der Index steigt für die Pflanzeichen um 0,06 und für die verpflanzten Eichen um 1,16 an. Einer signifikant höheren Konkurrenz sind die beherrschten Eichen im Gegensatz zu den herrschenden ausgesetzt. Bei Ersteren ist ein um 0,58 höherer HEGYI-Index zu finden als bei Letzteren.

Tabelle 17: Konkurrenzbeschreibende Daten; Holzminden, 22 jährige Eiche (HEGYI-Index, Baumabstand der nächsten drei Nachbarn zum Probebaum); der Stern * beschreibt die signifikanten Unterschiede ( $f$-Test) des HEGYI-Index $(p=0,05)$ in Richtung der Pfeile

\begin{tabular}{|c|c|c|c|c|c|c|c|c|}
\hline & Hegyi & $\begin{array}{c}\text { Pflanzung } \\
*\end{array}$ & $\begin{array}{r}\text { Saat } \\
*\end{array}$ & Verpfl & & & $\stackrel{\longrightarrow}{\longrightarrow}$ & Saat \\
\hline \multirow[t]{2}{*}{ Kraft 2} & HEGYI & 2,25 & 2,19 & 3,35 & Kraft 4 & HEGYI & 3,41 & 3,99 \\
\hline & Nachbar 1 & 1,19 & 0,99 & 0,90 & & Nachbar 1 & 1,02 & 0,91 \\
\hline \multirow[t]{2}{*}{ Abstände (m) } & Nachbar 2 & 1,39 & 1,36 & 1,04 & & Nachbar 2 & 1,51 & 1,27 \\
\hline & Nachbar 3 & 1,54 & 1,61 & 1,31 & & Nachbar 3 & 1,53 & 1,51 \\
\hline
\end{tabular}

\section{Kattenbühl}

Die Konkurrenzindizes von Kattenbühl variieren stark und lassen nicht den Schluß zu, daß mit Abnahme der sozialen Stellung der HEGYI-INDEX und damit die Konkurrenz zunimmt. Die Ursachen der Variation liegen vermutlich darin, daß die Eichen jünger sind als die der anderen Bestände. Eine andere Möglichkeit bestände darin, daß in Kattenbühl häufig durch den Einsatz von Kindern des naheliegenden Waldjugendheims bestandespflegende Eingriffe erfolgten.

Tabelle 18: Konkurrenzbeschreibende Daten; Kattenbühl, 13 jährige Eiche (HEGYI-Index, Baumabstand der nächsten drei Nachbarn zum Probebaum)

\begin{tabular}{|ccccc|}
\hline & & Pflanzung & Saat I & Saat II \\
\hline Kraft 1 & HEGYI & 1,68 & 5,52 & 3,89 \\
& Nachbar 1 & 0,87 & 0,40 & 0,23 \\
Abstände (m) & Nachbar 2 & 1,04 & 1,00 & 0,65 \\
& Nachbar 3 & 1,35 & 1,02 & \\
\hline \multirow{2}{*}{ Kraft 2 } & HEGYI & 2,05 & 2,26 & 4,86 \\
& Nachbar 1 & 0,90 & 0,64 & 0,18 \\
Abstände (m) & Nachbar 2 & 1,16 & 0,74 & 0,18 \\
& Nachbar 3 & 1,93 & 1,20 & 0,10 \\
\hline Kraft 3 & HEGYI & 4,20 & 4,34 & 2,48 \\
& Nachbar 1 & 0,98 & 0,60 & 0,35 \\
Abstände (m) & Nachbar 2 & 1,12 & 0,66 & 0,60 \\
& Nachbar 3 & 1,35 & 1,03 & \\
\hline Kraft 4 & HEGYI & 4,66 & 18,47 & 5,84 \\
& Nachbar 1 & 1,13 & 0,49 & 0,45 \\
Abstände (m) & Nachbar 2 & 1,00 & 0,79 & 0,90 \\
& Nachbar 3 & 1,40 & 0,54 & \\
\hline
\end{tabular}




\subsubsection{2 Überragungswinkel}

Eine andere Möglichkeit für die Beschreibung der Konkurrenzsituation stellt ein Höhenvergleich zwischen Probebaum und Nachbarbäumen dar. Für die Fichten und Buchen aus Holzerode wurden alle Höhen der Nachbarbäume ermittelt, die die Krone des Probebaumes berührten. Mit diesen Daten wird der Überragungswinkel berechnet. In Tabelle 19 ist für die Fichten und Buchen der Überragungswinkel (Summe $\alpha$ ), die Anzahl der Höhenbedränger und der Überragungswinkel pro Bedränger, dargestellt. In allen Beständen steigen mit Abnahme der sozialen Stellung sowohl die Summe der Überragungswinkel als auch der Überragungswinkel pro Bedränger an.

Insgesamt wird die gemischte Buche am stärksten durch höhere und nähere Nachbarn bedrängt. Der bedrängende einzelne Nachbarbaum der herrschenden Buchen im Mischbestand hat einen rund $10^{\circ}$ größeren Winkel, als das bei der herrschenden Mischbestandsfichte und der herrschenden Buche im Reinbestand der Fall ist. Die Reinbestandsfichte zeigt gegenüber der Buche im Mischbestand eine $3^{\circ}$ geringere Abweichung bei jedoch durchschnittlich nur einem Konkurrenzbaum.

Tabelle 19: Summe der Überragungswinkel als Maß der Konkurrenz; Holzerode, Buche und Fichte. Der Stern * beschreibt die signifikanten Unterschiede (f-Test) des Überragungswinkels $(p=0,05)$ in Richtung der Pfeile

\begin{tabular}{|c|c|c|c|c|c|}
\hline Kraft & $\begin{array}{l}\text { Konkurrenzbeschreibende Faktoren } \\
\text { Überragungswinkel }\end{array}$ & Buche & $\begin{array}{l}\mathrm{Bu} / \mathrm{Fi} \\
-*\end{array}$ & $\stackrel{\text { Fichte }}{\longrightarrow}$ & $\stackrel{\mathrm{Fi} / \mathrm{Bu}}{\longrightarrow}$ \\
\hline \multirow[t]{3}{*}{1} & Überragungswinkel $\left({ }^{\circ}\right)$ & & & & 42,98 \\
\hline & Anzahl Bedränger & & & & 2,00 \\
\hline & Überragungswinkel pro Bedränger $\left({ }^{\circ}\right)$ & & & & 21,49 \\
\hline \multirow[t]{3}{*}{2} & Überragungswinkel $\left({ }^{\circ}\right)$ & 28,08 & 141,62 & 28,57 & 82,73 \\
\hline & Anzahl Bedränger & 1,25 & 4,00 & 1,00 & 4,25 \\
\hline & Überragungswinkel pro Bedränger $\left(^{\circ}\right)$ & 20,74 & 29,16 & 25,80 & 20,97 \\
\hline \multirow[t]{3}{*}{3} & Überragungswinkel $\left({ }^{\circ}\right)$ & 82,60 & 241,68 & & 106,03 \\
\hline & Anzahl Bedränger & 2,80 & 5,89 & & 3,00 \\
\hline & Überragungswinkel pro Bedränger $\left({ }^{\circ}\right)$ & 30,35 & 43,22 & & 43,87 \\
\hline \multirow[t]{3}{*}{4} & Überragungswinkel $\left(^{\circ}\right)$ & & & & 155,41 \\
\hline & Anzahl Bedränger & & & & 3,00 \\
\hline & Überragungswinkel pro Bedränger $\left({ }^{\circ}\right)$ & & & & 54,94 \\
\hline
\end{tabular}

Die drei Faktoren

- HeGYI-Index

- durchschnittliche Baumentfernung

- und die Summe der Überragungswinkel

belegen, daß die Buche des Mischbestandes einer deutlich höheren Konkurrenz ausgesetzt ist als die anderen Kollektive. Im weiteren wird getrennt unterschieden zwischen Durchmesserbetrachtung (HEGYI-Index) und Höhenbetrachtung (Überragungswinkel). Dabei weisen die Fichten im Mischbestand den geringsten HEGYI-Index, jedoch nahezu den höchsten Überragungswinkel auf. 


\subsubsection{Probebaumbeschreibende Parameter (BHD, Höhe, H/D-Wert, Stammvolu- men) Anzahl der Probebäume und soziale Stellung}

\section{Holzerode}

In Tabelle 20 sind die mittleren oberirdischen Baumparameter mit jeweiliger Standardabweichung aufgeführt. Die Fichte im Reinbestand ist im Mittel nicht dicker als die im Mischbestand, wohl aber signifikant höher. Bei der Buche unterscheidet sich der BHD der Probebäume im Rein- und im Mischbestand kaum voneinander. Dabei ist die Buche im Reinbestand signifikant höher als die im Mischbestand. In Anhang IV sind die Baumparameter, getrennt nach sozialer Stellung, aufgeführt.

Die ältesten Bäume mit dem durchschnittlich geringsten HD-Wert sind bei dem Fichten/Buchenbestand zu finden. Es werden in diesem Kollektiv sechs Bäume der Kraft 'schen Klassen 1 und 2 ausgewählt, und fünf der Klassen 3 und 4. Dadurch ist die Standardabweichung der Fichte im Mischbestand höher als bei den anderen Beständen, die eine geringere Anzahl sozialer Klassen aufweisen. Die Reinbestandsbäume sind im Mittel zehn Jahre jünger als die Mischbestandsbäume, außerdem weisen sie einen höheren HD-Wert auf. Hierbei sind bei Abnahme des sozialen Status die Bäume im Mittel zehn Jahre jünger. Bei den Buchen im Mischbestand steigt das Alter von der zweiten zur dritten Kraft'schen Klasse um rund vier Jahre an, der HD-Wert hingegen differiert kaum. Mit $88 \%$ sind bei diesem Bestand die meisten Bäume in der dritten Klasse zu finden. Das Stammvolumen ist bei den Fichten nahezu doppelt so hoch wie bei den Buchen. Wie auch schon bei den anderen Größen zeigt die Mischbestandsfichte ein größeres Stammvolumen als die Reinbestandsfichte (20\%). Die Reinbestandsbuche weist ein $25 \%$ höheres Volumen auf als die Mischbestandsbuche.

Tabelle 20: Mittlere Probebaumparameter $x$ und Standardabweichungen (sx); Holzerode, Fichte und Buche im Rein- und Mischbestand (55-65jährig). Der Stern* beschreibt die signifikanten Unterschiede (f-Test) des Überragungswinkels ( $p=$ 0,05) in Richtung der Pfeile

\begin{tabular}{|lcccccccc|}
\hline & Buche & \multicolumn{3}{c}{ Buche / Fichte } & \multicolumn{2}{c|}{ Fichte } & \multicolumn{3}{c|}{ Fichte / Buche } \\
& $\mathbf{x}$ & $(\mathrm{sx})$ & $\mathbf{x}$ & $(\mathrm{sx})$ & $\mathbf{x}$ & $(\mathrm{sx})$ & $\mathbf{x}$ & $(\mathrm{sx})$ \\
\hline Alter & 59,7 & $(4,6)$ & 60,4 & $(6,2)$ & 55,4 & $(1,2)$ & 65,4 & $(6,1)$ \\
BHD $(\mathrm{cm})$ & 21,2 & $(2,6)$ & 21,7 & $(4,9)$ & 27,8 & $(2,9)$ & 27,9 & $(7,0)$ \\
Höhe $(\mathrm{m})$ & $24,8^{*}$ & $(1,4) \rightarrow 22,1$ & $(2,5)$ & $28,0^{*}$ & $(2,7) \rightarrow 24,1$ & $(2,9)$ \\
H/D-Wert & 118,3 & $(12,6)$ & 105,1 & $(19,7)$ & 100,8 & $(2,2)$ & 89,9 & $(15,6)$ \\
Stammvolumen $\left(\mathrm{m}^{3}\right)$ & 0,44 & $(0,12)$ & 0,32 & $(0,16)$ & 0,71 & $(0,14)$ & 0,73 & $(0,36)$ \\
Probebäume $(\mathrm{n})$ & 10 & & 12 & & 10 & & 11 & \\
Soziale Stellung & $2 ; 3$ & & $1,2,3$ & & 2 & & $1,2,3,4$ & \\
\hline
\end{tabular}




\section{Krebeck}

Wie Tabelle 21 zeigt, steigen mit zunehmendem Alter die H/D-Werte der Fichten an. Bei den Bäumen der Kraft'schen Klassen 1 und 2 liegen sie unter 90. Eine Ausnahme bilden die Fichten der zweiten Kraft'schen Klasse des 41 jährigen Bestandes, deren H/D-Wert auf über 100 ansteigt. Bei den mitherrschenden und den beherrschten Bäumen liegen die Werte durchgängig über 100. Bei den 41 jährigen beherrschten Fichten steigen sie auf bis rund 150 an.

Tabelle 21: Mittlere Probebaumparameter $\boldsymbol{x}$ und Standardabweichungen (sx); Krebeck, 21-, 31- und 41 jährige Fichte im Reinbestand

\begin{tabular}{|c|c|c|c|c|c|c|c|}
\hline \multirow[t]{2}{*}{ Fläche } & & \multicolumn{2}{|c|}{ Fichte 21} & \multicolumn{2}{|c|}{ Fichte 30} & \multicolumn{2}{|c|}{ Fichte 41} \\
\hline & & $\mathbf{x}$ & $(s x)$ & $\mathbf{x}$ & (sx) & $\mathbf{x}$ & $(s x)$ \\
\hline \multirow[t]{5}{*}{ Kraft 1} & $\mathrm{BHD}(\mathrm{cm})$ & 15,1 & $(0,8)$ & 20,1 & $(1,7)$ & 23,8 & $(1,6)$ \\
\hline & Höhe (m) & 11,5 & $(0,9)$ & 14,9 & $(0,6)$ & 21,3 & $(1,0)$ \\
\hline & H/D-Wert & 76,5 & $(2,3)$ & 74,4 & $(5,2)$ & 89,7 & $(3,8)$ \\
\hline & Stammvolumen $\left(\mathrm{m}^{3}\right)$ & 0,1 & $(0,0)$ & 0,2 & $(0,1)$ & 0,4 & $(0,0)$ \\
\hline & Probebäume (n) & 4 & & 4 & & 5 & \\
\hline \multirow[t]{5}{*}{ Kraft 2} & $\mathrm{BHD}(\mathrm{cm})$ & 12,3 & $(1,3)$ & 17,4 & $(1,7)$ & 19,7 & $(1,6)$ \\
\hline & Höhe (m) & 10,1 & $(0,3)$ & 14,4 & $(1,7)$ & 20,4 & $(0,9)$ \\
\hline & H/D-Wert & 82,7 & $(8,0)$ & 87,8 & $(15,5)$ & 104,1 & $(7,1)$ \\
\hline & Stammvolumen $\left(\mathrm{m}^{3}\right)$ & 0,1 & $(0,0)$ & 0,2 & $(0,1)$ & 0,3 & $(0,0)$ \\
\hline & Probebäume (n) & 3 & & 4 & & 5 & \\
\hline \multirow[t]{5}{*}{ Kraft 3} & $\mathrm{BHD}(\mathrm{cm})$ & 9,9 & $(0,9)$ & 12,4 & $(1,7)$ & 14,0 & $(2,1)$ \\
\hline & Höhe (m) & 10,2 & $(0,7)$ & 14,2 & $(3,0)$ & 16,2 & $(1,0)$ \\
\hline & H/D-Wert & 102,7 & $(3,4)$ & 113,8 & $(13,3)$ & 116,2 & $(10,5)$ \\
\hline & Stammvolumen $\left(\mathrm{m}^{3}\right)$ & 0,04 & $(0,0)$ & 0,1 & $(0,1)$ & 0,1 & $(0,0)$ \\
\hline & Probebäume (n) & 4 & & 4 & & 2 & \\
\hline \multirow[t]{5}{*}{ Kraft 4} & $\mathrm{BHD}(\mathrm{cm})$ & 6,8 & $(1,0)$ & 8,7 & $(1,5)$ & 9,8 & $(0,6)$ \\
\hline & Höhe (m) & 7,5 & $(1,0)$ & 11,2 & $(2,0)$ & 15,1 & $(0,8)$ \\
\hline & H/D-Wert & 111,1 & $(12,8)$ & 129,2 & $(0,7)$ & 153,6 & $(1,9)$ \\
\hline & Stammvolumen $\left(\mathrm{m}^{3}\right)$ & 0,02 & $(0,001)$ & 0,01 & $(0,001)$ & 0,1 & $(0,01)$ \\
\hline & Probebäume (n) & 4 & & 3 & & 3 & \\
\hline
\end{tabular}




\section{Knesebeck}

Die nach sozialer Stellung differenzierten Probebaumdaten in Tabelle 22 zeigen für die Fichte und für die Kiefer die erwartete Zunahme der Höhe und des BHD mit Anstieg des Alters und der sozialen Stellung. Das H/D-Verhältnis steigt mit Minderung der Kraft'schen Klasse bei den älteren Beständen an. Die Bäume der 20 jährigen Flächen zeigen dieses Merkmal nicht. Die Ursachen hierfür liegen in dem Bestandesaufbau. Weiterhin wird geprüft, ob Unterschiede zwischen den Baumarten bestehen. Hierbei zeigt die Fichte für nahezu alle Kraft'schen Klassen für BHD, Höhe und Stammvolumen höhere Werte als die Kiefer.

Im Altersvergleich der Bestandesformen weisen die um 30 jährigen Fichten durchgängig höhere H/D-Werte auf als die des 50 jährigen Bestandes.

Tabelle 22: Mittlere Probebaumparameter $x$ und Standardabweichungen (sx); Knesebeck, 2050 jährige Fichte und Kiefer im Reinbestand

\begin{tabular}{|c|c|c|c|c|c|c|c|}
\hline \multicolumn{2}{|c|}{ Fläche } & \multirow{2}{*}{$\begin{array}{r}\text { Fi } 20 \\
x \\
\end{array}$} & \multirow{2}{*}{$\begin{array}{r}\text { Fi } 31 \\
x\end{array}$} & Fi 50 & \multirow{2}{*}{$\begin{array}{l}\text { Ki } 20 \\
\quad x(s x)\end{array}$} & \multirow{2}{*}{$\begin{array}{l}\text { Ki } 33 \\
\quad \times(s x)\end{array}$} & \multirow{2}{*}{$\begin{array}{l}\mathrm{Ki} 47 \\
\mathrm{x}(\mathrm{sx})\end{array}$} \\
\hline & & & & $\mathbf{x}(\mathbf{s x})$ & & & \\
\hline \multirow{5}{*}{ 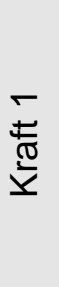 } & $\mathrm{BHD}(\mathrm{cm})$ & $9,8 \quad(0,4)$ & $21,0(1,6)$ & $30,1 \quad(2,7)$ & $8,7(1,5)$ & $17,1(1,0)$ & $26,9(0,8)$ \\
\hline & Höhe (m) & $8,1 \quad(0,2)$ & $16,9(0,1)$ & $18,4 \quad(1,5)$ & $8,1(2,0)$ & $13,4(0,6)$ & $17,5(0,9)$ \\
\hline & $\mathrm{H} / \mathrm{D}$-Wert & $82,6(0,8)$ & $80,7 \quad(6,5)$ & $61,3 \quad(4,7)$ & $95,1(22,8)$ & $78,4 \quad(7,9)$ & $65,1(4,6)$ \\
\hline & Stammvolumen $\left(\mathrm{m}^{3}\right)$ & $0,03(0,0)$ & $0,3(0,0)$ & $0,6 \quad(0,1)$ & $0,03(0,01)$ & $0,2 \quad 0,01$ & $0,5(0,1)$ \\
\hline & Probebäume (n) & 2 & 4 & 4 & 3 & 4 & 5 \\
\hline \multirow{5}{*}{ 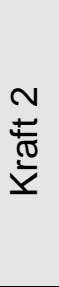 } & $\mathrm{BHD}(\mathrm{cm})$ & $7,2(0,3)$ & $15,8(0,5)$ & $24,2 \quad(2,3)$ & $6,3(0,6)$ & $12,3(1,6)$ & $22,1(1,1)$ \\
\hline & Höhe (m) & $6,2(0,3)$ & $15,2(0,6)$ & $18,9 \quad(0,6)$ & $5,9(0,4)$ & $13,2(1,2)$ & $17,1(0,6)$ \\
\hline & H/D-Wert & $86,2(6,4)$ & $96,5(4,5)$ & $78,7 \quad(5,7)$ & $94,1(9,9)$ & $108,0(6,0)$ & $77,3(6,0)$ \\
\hline & Stammvolumen $\left(\mathrm{m}^{3}\right)$ & $0,1 \quad(0,1)$ & $0,2(0,0)$ & $0,4 \quad(0,1)$ & $0,01(0,0)$ & $0,1(0,02)$ & $0,3(0,05)$ \\
\hline & Probebäume (n) & 3 & 4 & 5 & 3 & 4 & 4 \\
\hline \multirow{5}{*}{$\begin{array}{l}\text { m } \\
\stackrel{ \pm}{\frac{\pi}{\pi}} \\
\frac{1}{\Sigma}\end{array}$} & $\mathrm{BHD}(\mathrm{cm})$ & $7,8 \quad(0,3)$ & $10,5(1,0)$ & $15,8 \quad(1,3)$ & $5,5(2,1)$ & $9,5(1,5)$ & $18,2(0,6)$ \\
\hline & Höhe (m) & $6,6(1,0)$ & $11,9(0,9)$ & $15,2 \quad(2,0)$ & $4,7(1,5)$ & $11,6(0,8)$ & $16,1(0,9)$ \\
\hline & H/D-Wert & $84,2(15,9)$ & $114,9(18,1)$ & $96,0(11,0)$ & $85,7(6,1)$ & $125,1(28,9)$ & $88,4(3,0)$ \\
\hline & Stammvolumen $\left(\mathrm{m}^{3}\right)$ & $0,02(0,0)$ & $0,2(0,2)$ & $0,3 \quad(0,0)$ & 0,01 & $0,02(0,0)$ & $0,2(0,01)$ \\
\hline & Probebäume (n) & 3 & 4 & 5 & 2 & 3 & 3 \\
\hline \multirow{5}{*}{ 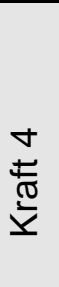 } & $\mathrm{BHD}(\mathrm{cm})$ & $4,2(1,8)$ & $7,9(1,3)$ & 11,5 & $4,5(0,5)$ & $7,1 \quad(0,4)$ & $13,0(2,8)$ \\
\hline & Höhe (m) & $4,1 \quad(1,4)$ & $10,0 \quad(1,2)$ & 11,0 & $4,4(1,2)$ & $9,8(1,3)$ & $15,0(2,0)$ \\
\hline & H/D-Wert & $100,6(17,2)$ & $127,3(8,6)$ & 95,7 & $96,7(15,3)$ & $138,7(26,5)$ & $116,7(11,6)$ \\
\hline & Stammvolumen $\left(\mathrm{m}^{3}\right)$ & $0,005(0,0)$ & $0,02(0,0)$ & 0,1 & $0,004(0,0)$ & $0,02(0,0)$ & $0,1(0,05)$ \\
\hline & Probebäume (n) & 3 & 4 & 1 & 3 & 5 & 3 \\
\hline
\end{tabular}

\section{Holzminden}

In Tabelle 23 sind die Mittelwerte der sozialen Stellungen 2 und 4 der rund 22 jährigen Eichen zusammengestellt. Die herrschenden Bäume des Saatbestandes sind signifikant dicker als die Eichen der anderen Bestände, und in der Höhe übertreffen sie diese um 4\%. Die gesäten Eichen besitzen somit ein signifikant höheres Stammvolumen. Der H/D-Wert ist bei den herrschenden Saateichen am geringsten. 
Bei den Eichen der Verpflanzung handelt es sich ausschließlich um herrschende Bäume. Die Höhen- und BHD- Mittelwerte sind im Vergleich zu den anderen Beständen niedrig, und der H/D-Wert ist sehr hoch. Mit über zehn Metern Höhe ist die verpflanzte Eiche $13 \%$ niedriger als die gesäte und $11 \%$ niedriger als die gepflanzte Eiche.

Die beherrschten Bäume der Pflanzung besitzen einen höheren BHD, eine geringere Höhe und damit einen geringeren H/D-Wert als die der Saat.

Tabelle 23: Mittlere Probebaumparameter $x$ und Standardabweichungen (sx); Holzminden, 22 jährige Eiche; Saat und Pflanzung. Der Stern * beschreibt die signifikanten Unterschiede (f-Test) des Überragungswinkels $(p=0,05)$ in Richtung der Pfeile

\begin{tabular}{|c|c|c|c|c|c|}
\hline \multirow[t]{2}{*}{ Fläche } & & \multicolumn{2}{|l|}{ Pflanzung } & Saat & Verpflanzung \\
\hline & & $\mathbf{x}(\mathbf{s x})$ & & $x(s x)$ & $\mathbf{x}(\mathbf{s x})$ \\
\hline \multirow[t]{5}{*}{ Kraft 2} & $\mathrm{BHD}(\mathrm{cm})$ & $10,0(0,9)$ & 4 & $11,2^{*}(1,0) \longrightarrow$ & $8,9(0,7)$ \\
\hline & Höhe (m) & $11,4(0,8)$ & & $11,8(0,3)$ & $10,3(1,1)$ \\
\hline & H/D-Wert & $114,2(10,7)$ & & $105,5(8,4)$ & $116,4(16,1)$ \\
\hline & Stammvolumen $\left(\mathrm{m}^{3}\right)$ & $0,04(0,01)$ & $\leftarrow$ & $0,05^{*}(0,01) \longrightarrow$ & $0,03(0,01)$ \\
\hline & Probebäume (n) & 7,0 & & 7,0 & 6,0 \\
\hline \multirow[t]{5}{*}{ Kraft 4} & $\mathrm{BHD}(\mathrm{cm})$ & $7,1(0,8)$ & & $6,8(0,7)$ & \\
\hline & Höhe (m) & $9,7(0,9)$ & 4 & $10,7^{*}(0,7)$ & \\
\hline & HD-Wert & $139,7(23,6)$ & & $158,5(18,7)$ & \\
\hline & Stammvolumen $\left(\mathrm{m}^{3}\right)$ & $0,02(0,01)$ & & $0,02(0,00)$ & \\
\hline & Probebäume $(n)$ & 7,0 & & 7,0 & \\
\hline
\end{tabular}

\section{Kattenbühl}

Die Probebäume der drei 13 jährigen Eichenflächen in Kattenbühl zeigen für den BHD in der Reihenfolge Pflanzung, Saat I und Saat II abfallende Werte. Die Höhe der Eichen differiert im Vergleich der drei Bestände. Im Durchschnitt aller 4 sozialen Klassen sind die Bäume der beiden Saatbestände $7 \%$ niedriger als die der Pflanzung.

Tabelle 24: Mittlere Probebaumparameter $\boldsymbol{x}$ und Standardabweichungen (sx); Kattenbühl, 13 jährige Eiche; Saat und Pflanzung

\begin{tabular}{|clccc|}
\hline Fläche & & $\begin{array}{c}\text { Pflanzung } \\
\mathbf{x}(\mathbf{s x})\end{array}$ & $\begin{array}{c}\text { Saat I } \\
\mathbf{x}(\mathbf{s x})\end{array}$ & $\begin{array}{r}\text { Saat II } \\
\mathbf{x}(\mathbf{s x})\end{array}$ \\
\cline { 2 - 5 } Kraft 1 & BHD (cm) & $8,6(0,5)$ & $8,0(0,7)$ & $6,9(1,5)$ \\
& Höhe (m) & $7,0(0,6)$ & $7,0(0,4)$ & $7,1(0,6)$ \\
& H/D-Wert & $82,4(8,7)$ & $87,4(3,1)$ & $105,5(14,2)$ \\
& Stammvolumen $\left(\mathrm{dm}^{3}\right)$ & $18,9(3,2)$ & $17,2(3,9)$ & $12,5(5,5)$ \\
& Probebäume $(\mathrm{n})$ & 5,0 & 4,0 & 4,0 \\
\hline Kraft 2 & BHD (cm) & $6,9(0,6)$ & $6,5(1,0)$ & $5,1(0,8)$ \\
& Höhe (m) & $6,8(1,1)$ & $6,1(0,6)$ & $5,8(0,4)$ \\
& H/D-Wert & $99,0(13,5)$ & $94,6(12,4)$ & $116,5(23,0)$ \\
& Stammvolumen $\left(\mathrm{dm}^{3}\right)$ & $12,1(3,1)$ & $10,0(2,6)$ & $6,1(1,9)$ \\
& Probebäume $(\mathrm{n})$ & 4,0 & 4,0 & 4,0 \\
\hline
\end{tabular}




\begin{tabular}{|llccr|}
\hline Fläche & & Pflanzung & Saat I & Saat II \\
& & $\mathbf{x}(\mathbf{s x})$ & $\mathbf{x}(\mathbf{s x})$ & $\mathbf{x}(\mathbf{s x})$ \\
Kraft 3 & $4,5(0,2)$ & $4,2(0,5)$ & $5,1(1,4)$ \\
& BHD $(\mathrm{cm})$ & $6,2(0,3)$ & $5,0(0,4)$ & $5,8(1,1)$ \\
& Höhe $(\mathrm{m})$ & $139,4(2,1)$ & $119,5(7,3)$ & $117,7(22,4)$ \\
& H/D-Wert & $5,4(1,3)$ & $4,2(0,9)$ & $6,9(5,1)$ \\
& Stammvolumen $\left(\mathrm{dm}^{3}\right)$ & 3,0 & 4,0 & 3,0 \\
\hline Kraft 4 & Probebäume $(\mathrm{n})$ & $3,2(0,7)$ & $3,3(0,5)$ & $2,9(0,4)$ \\
& BHD $(\mathrm{cm})$ & $4,4(0,8)$ & $4,6(0,2)$ & $4,4(0,5)$ \\
& Höhe $(\mathrm{m})$ & $140,0(22,0)$ & $144,5(20,2)$ & $150,1(18,1)$ \\
& H/D-Wert & $2,4(1,2)$ & $2,5(0,7)$ & $2,0(0,6)$ \\
& Stammvolumen $\left(\mathrm{dm}^{3}\right)$ & 4,0 & 3,0 & 4,0 \\
\hline
\end{tabular}

\subsubsection{Kronenparameter}

In den jeweiligen Tabellen werden für die untersuchten Baumarten Mittelwerte $\mathbf{x}$ einschließlich Standardabweichung (sx) aufgeführt. Die Gewichtsangaben der Krone gelten für die Trockensubstanz.

\section{Holzerode}

Die Buche zeigt in Mischung ein $54 \%$ höheres Kronengewicht, eine $60 \%$ größere Kronenschirmfläche und ein $35 \%$ höheres Kronenprozent als im Reinbestand (Tabelle 25). Das hohe Kronengewicht der Mischbestandsbuche unterliegt einer höheren Streuung als das der Buche im Reinbestand. Signifikante Unterschiede sind jedoch nicht vorhanden.

Die Fichten haben im Reinbestand eine rund 50\% schwerere Krone als die Buchen (signifikant). Die Kronenschirmfläche differiert im Vergleich Buche zur Fichte durchschnittlich um $34 \%$. Die Mischbestandsfichte weist rund 35\% weniger Kronenmasse, eine $70 \%$ kürzere Krone, jedoch eine $20 \%$ größere Kronenüberschirmungsfläche auf als die Reinbestandsfichte. Weiterhin besitzt die Fichte im Reinbestand ein Kronenprozent von nahezu $50 \%$ und die im Mischbestand nur eins von rund $30 \%$.

Tabelle 25: Mittlere Probebaumparameter $x$ und Standardabweichungen (sx); Holzerode, Buche und Fichte, 55-65 jährig im Rein- und im Mischbestand

\begin{tabular}{|lcccc|}
\hline Fläche & Buche & Buche / Fichte & Fichte & Fichte / Buche \\
& $\mathbf{x ~ ( s x )}$ & $\mathbf{x ~ ( s x )}$ & $\mathbf{x ~ ( s x )}$ & $\mathbf{x}(\mathbf{s x})$ \\
\hline Kronengewicht $(\mathrm{kg})$ & $18,4(7,0)$ & $40,0(23,9)$ & $56,6(19,1)$ & $43,1(18,6)$ \\
Kronenansatz $(\mathrm{m})$ & $15,1(3,0)$ & $8,8(3,6)$ & $14,5(1,3)$ & $16,2(2,6)$ \\
Kronenlänge $(\mathrm{m})$ & $9,8(2,5)$ & $13,3(2,5)$ & $13,5(3,0)$ & $8,0(2,5)$ \\
Kronenprozent \% & $39,5(10,3)$ & $61,0(13,6)$ & $48,8(6,2)$ & $33,2(6,3)$ \\
Kronenschirmfläche $\left(\mathrm{m}^{2}\right)$ & $16,4(7,0)$ & $43,7(18,7)$ & $9,4(2,2)$ & $11,2(11,2)$ \\
\hline
\end{tabular}




\section{Krebeck}

Das Kronentrockengewicht (gesamt) fällt von der ersten zur vierten sozialen Stellung ab (Tabelle 26). Die Abweichungen der ersten Kraft'schen Klassen vom jeweiligen Gesamtmittelwert betragen für die Fichten des 21- und 41 jährigen Bestandes etwa $70 \%$, für den 30 jährigen Bestand $90 \%$ in Richtung der vorherrschenden und mitherrschenden Bäume. Entsprechende Abweichungen der vierten Klasse vom Mittel liegen bei den 21 jährigen bei $60 \%$, bei den 30- und 41 jährigen Fichten bei $80 \%$. Bei Betrachtung der drei Altersklassen weisen die Bäume des 30 jährigen Bestandes das höchste Kronengewicht auf. Differenziert nach Ast / Ästchen / Nadel ist für die untersuchten Altersstufen der Anteil der Nadelmasse am Kronengewicht am höchsten. Das größte Kronenprozent zeigt über alle sozialen Stellungen der junge, das kleinste der älteste Bestand.

Tabelle 26: Mittlere Probebaumparameter $\boldsymbol{x}$ und Standardabweichungen (sx); Krebeck, 2141 jährige Fichte im Reinbestand

\begin{tabular}{|c|c|c|c|c|}
\hline & & Fichte 21 & Fichte 30 & Fichte 41 \\
\hline & & $\mathbf{x}(\mathbf{s x})$ & $\mathbf{x}(\mathbf{s x})$ & $x(s x)$ \\
\hline \multirow[t]{7}{*}{ Kraft 1} & Kronengewicht (kg) & $28,0(5,6)$ & $57,7(21,9)$ & $46,87(9,7)$ \\
\hline & - Ast (kg) & $8,0(2,3)$ & $16,4(7,5)$ & $14,1(4,0)$ \\
\hline & - Ästchen (kg) & $5,3(0,7)$ & $11,3(3,1)$ & $9,9(3,1)$ \\
\hline & - Blatt (kg) & $14,8(3,4)$ & $30,0(11,6)$ & $22,9(9,3)$ \\
\hline & Kronenansatz (m) & $2,0(0,6)$ & $6,1(0,9)$ & $10,5(1,2)$ \\
\hline & Kronenlänge (m) & $9,5(1,3)$ & $8,8(1,0)$ & $10,8(1,0)$ \\
\hline & Kronenprozent (\%) & $82,6(6,1)$ & $59,1(6,1)$ & $50,8(4,6)$ \\
\hline \multirow[t]{7}{*}{ Kraft 2} & Kronengewicht (kg) & $23,3(3,5)$ & $33,5(11,6)$ & $29,0(9,1)$ \\
\hline & - Ast (kg) & $6,9(2,0)$ & $8,5(4,0)$ & $9,3(3,6)$ \\
\hline & - Ästchen (kg) & $4,4(0,1)$ & $7,3(2,8)$ & $6,3(1,8)$ \\
\hline & - Blatt $(\mathrm{kg})$ & $12,0(1,8)$ & $17,7(5,5)$ & $13,5(3,8)$ \\
\hline & Kronenansatz (m) & $1,9(0,3)$ & $6,8(0,7)$ & $10,7(0,9)$ \\
\hline & Kronenlänge (m) & $8,2(0,4)$ & $8,3(1,9)$ & $9,8(1,6)$ \\
\hline & Kronenprozent (\%) & $81,2(2,8)$ & $54,4(6,9)$ & $47,6(6,1)$ \\
\hline \multirow[t]{7}{*}{ Kraft 3} & Kronengewicht $(\mathrm{kg})$ & $9,6(2,9)$ & $15,3(4,8)$ & $11,1(0,4)$ \\
\hline & - Ast (kg) & $2,7(1,2)$ & $5,8(5,5)$ & $2,8(0,1)$ \\
\hline & - Ästchen (kg) & $1,8(0,6)$ & $4,9(3,8)$ & $2,3(0,4)$ \\
\hline & - Blatt (kg) & $5,1(1,4)$ & $14,2(12,5)$ & $5,9(0,1)$ \\
\hline & Kronenansatz (m) & $3,3(1,0)$ & $6,6(1,4)$ & $10,7(0,2)$ \\
\hline & Kronenlänge (m) & $6,9(0,6)$ & $7,5(1,6)$ & $5,5(1,2)$ \\
\hline & Kronenprozent (\%) & $67,7(8,1)$ & $53,2(0,7)$ & $33,9(5,4)$ \\
\hline \multirow[t]{7}{*}{ Kraft 4} & Kronengewicht (kg) & $6,4(3,3)$ & $6,5(1,2)$ & $5,8(1,9)$ \\
\hline & - Ast (kg) & $1,8(1,0)$ & $1,5(0,4)$ & $1,6(1,0)$ \\
\hline & - Ästchen (kg) & $1,2(0,6)$ & $1,3(0,0)$ & $1,5(0,2)$ \\
\hline & - Blatt (kg) & $3,3(1,7)$ & $3,7(1,2)$ & $2,7(0,7)$ \\
\hline & Kronenansatz (m) & $2,0(0,3)$ & $6,0(0,5)$ & $9,9(0,7)$ \\
\hline & Kronenlänge (m) & $5,5(0,7)$ & $5,2(1,9)$ & $5,2(0,3)$ \\
\hline & Kronenprozent (\%) & $73,1(1,8)$ & $45,6(9,0)$ & $34,2(1,9)$ \\
\hline
\end{tabular}




\section{Knesebeck}

Das Kronengewicht steigt mit Verbesserung der sozialen Stellung an (Tabelle 27). Mit zunehmendem Bestandesalter von 20 auf rund 30 Jahre fällt das Kronenprozent ab. Zwischen 30 und 50 Jahren bleibt es nahezu gleich.

Beim Vergleich der Kiefer mit der Fichte wird der artspezifische Kronenaufbau deutlich. Die Kiefer weist ein ein- bis zweifach geringeres Kronengewicht sowie durchschnittlich ein um $30 \%$ geringeres Kronenprozent auf.

Tabelle 27: Mittlere Probebaumparameter $\boldsymbol{x}$ und Standardabweichungen (sx); Knesebeck, 2050 jährige Fichte und Kiefer im Reinbestand

\begin{tabular}{|c|c|c|c|c|c|c|c|}
\hline & Fichte 20 & Fichte 31 & Fichte 50 & Kiefer 20 & Kiefer 33 & Kiefer 47 \\
\hline & & $x(s x)$ & $\mathbf{x}(s \mathrm{x})$ & $x(s x)$ & $x(s x)$ & $x(s x)$ & $x(s x)$ \\
\hline \multirow{7}{*}{$\begin{array}{l}7 \\
\pm\end{array}$} & Kronengewicht (kg) & $11,4(1,5)$ & $59,8(19,2)$ & $100,9(17,7)$ & $4,6(1,9)$ & $18,9(3,0)$ & $44,5(10,5)$ \\
\hline & - Ast (kg) & & $26,4(1,8)$ & $49,2(5,7)$ & $1,5(1,4)$ & $7,9(1,7)$ & $19,0(3,9)$ \\
\hline & - Ästchen (kg) & & $10,9(1,6)$ & $22,4(3,7)$ & $0,3(0,3)$ & $2,9(0,7)$ & $3,8(0,6)$ \\
\hline & • Blatt (kg) & & $22,5(19,0)$ & $29,3(8,7)$ & $1,6(1,6)$ & $8,2(1,9)$ & $21,6(6,4)$ \\
\hline & Kronenansatz (m) & $0,8(0,4)$ & $6,7(1,6)$ & $6,2(1,9)$ & $3,5(1,2)$ & $8,3(0,9)$ & $10,9(0,9)$ \\
\hline & Kronenlänge (m) & $7,3(0,6)$ & $10,2(1,7)$ & $12,3(0,5)$ & $4,6(0,9)$ & $5,1(0,8)$ & $6,5(1,2)$ \\
\hline & Kronenprozent (\%) & $90,0(5,5)$ & $60,6(9,8)$ & $67,1(7,4)$ & $57,1(6,4)$ & $38,2(6,0)$ & $37,3(5,6)$ \\
\hline \multirow{7}{*}{$\begin{array}{l}N \\
\frac{ \pm}{\sigma} \\
\frac{\pi}{\grave{\Sigma}}\end{array}$} & Kronengewicht (kg) & $7,8(1,6)$ & $20,0(9,8)$ & $57,7(19,2)$ & $2,2(0,4)$ & $6,6(2,9)$ & $25,7(8,7)$ \\
\hline & • Ast (kg) & $4,2(0,1)$ & $9,3(3,9)$ & $31,0(11,1)$ & $0,2(0,4)$ & $2,5(0,5)$ & $10,8(4,8)$ \\
\hline & - Ästchen $(\mathrm{kg})$ & $1,6(0,1)$ & $4,4(2,6)$ & $13,9(6,0)$ & $0,0(0,1)$ & $0,9(0,2)$ & $2,4(1,3)$ \\
\hline & • Blatt (kg) & $2,9(0,6)$ & $6,4(3,5)$ & $12,8(2,9)$ & $0,3(0,5)$ & $3,3(2,7)$ & $12,5(3,2)$ \\
\hline & Kronenansatz (m) & $0,5(0,2)$ & $5,5(2,2)$ & $8,0(0,7)$ & $2,4(0,2)$ & $9,0(1,1)$ & $10,9(0,5)$ \\
\hline & Kronenlänge (m) & $5,7(0,3)$ & $9,7(2,0)$ & $10,9(0,2)$ & $3,5(0,3)$ & $4,2(1,2)$ & $6,2(0,4)$ \\
\hline & Kronenprozent (\%) & $92,4(2,5)$ & $64,1(14,1)$ & $57,7(2,2)$ & $59,6(1,1)$ & $31,6(8,1)$ & $36,2(1,9)$ \\
\hline \multirow{7}{*}{$\begin{array}{l}m \\
\frac{ \pm}{\pi} \\
\frac{\pi}{4}\end{array}$} & Kronengewicht (kg) & $6,1(1,1)$ & $8,5(3,0)$ & $27,6(4,2)$ & $2,4(1,8)$ & $3,3(1,6)$ & $19,4(4,8)$ \\
\hline & • Ast (kg) & & $4,4(1,6)$ & $15,8(2,0)$ & & $1,2(0,9)$ & $7,3(1,9)$ \\
\hline & • Ästchen (kg) & & $2,0(0,8)$ & $5,8(1,0)$ & & $0,5(0,4)$ & $1,8(0,4)$ \\
\hline & • Blatt (kg) & & $2,0(0,7)$ & $6,0(1,8)$ & & $1,6(0,7)$ & $10,3(2,8)$ \\
\hline & Kronenansatz (m) & $0,9(0,2)$ & $5,2(1,4)$ & $6,3(1,7)$ & $2,2(0,3)$ & $8,6(0,6)$ & $10,1(1,0)$ \\
\hline & Kronenlänge (m) & $5,6(0,9)$ & $6,7(1,2)$ & $8,8(1,7)$ & $3,6(1,8)$ & $3,0(0,4)$ & $6,0(1,9)$ \\
\hline & Kronenprozent (\%) & $85,8(2,4)$ & $56,4(11,1)$ & $58,5(8,6)$ & $80,7(25,2)$ & $25,7(2,3)$ & $36,8(9,8)$ \\
\hline \multirow{7}{*}{$\begin{array}{l}\dot{ \pm} \\
\frac{\pi}{\pi} \\
\frac{\pi}{2}\end{array}$} & Kronengewicht (kg) & $3,0(2,4)$ & $5,5(3,4)$ & 12,2 & $0,7(0,4)$ & $1,6(0,6)$ & $10,8(0,7)$ \\
\hline & • Ast (kg) & $1,7(1,5)$ & $3,0(2,0)$ & 6,5 & $0,1(0,2)$ & $0,5(0,2)$ & $4,8(0,6)$ \\
\hline & - Ästchen (kg) & $0,6(0,5)$ & $1,1(0,7)$ & 2,5 & $0,0(0,1)$ & $0,4(0,3)$ & $0,9(0,1)$ \\
\hline & • Blatt (kg) & $0,7(0,4)$ & $1,3(0,8)$ & 3,2 & $0,2(0,3)$ & $0,7(0,3)$ & $5,1(0,3)$ \\
\hline & Kronenansatz (m) & $0,5(0,2)$ & $4,1(1,5)$ & 4,5 & $2,2(0,9)$ & $7,8(1,0)$ & $9,8(0,9)$ \\
\hline & Kronenlänge (m) & $3,6(1,2)$ & $5,9(2,6)$ & 6,5 & $2,2(0,5)$ & $2,0(0,6)$ & $5,2(1,4)$ \\
\hline & Kronenprozent (\%) & $88,3(3,0)$ & $57,2(21,6)$ & 59,1 & $49,9(8,0)$ & $20,0(4,4)$ & $34,1(6,4)$ \\
\hline
\end{tabular}




\section{Holzminden}

Tabelle 28 stellt die Kronenparameter der 22 jährigen Eichenbestände dar. Die Eiche der Verpflanzung zeigt bei dem höchsten Kronengewicht den niedrigsten Kronenansatz. Alle Bestände besitzen mehr Ast- als Blatt- oder Ästchenmasse.

Die Eichen der Pflanzung Kraft 2 weisen im Vergleich zu den anderen herrschenden Bäumen das geringste Kronenprozent auf. Die beherrschten Bäume zeigen rund $80 \%$ weniger Kronengewicht als die herrschenden. Die Saateichen besitzen in der Relation ein über $50 \%$ höheres Kronengewicht in der zweiten Klasse als die gepflanzten Eichen. Die beherrschten Saatbäume weisen ein rund $50 \%$ geringeres Kronenprozent bei einer fast siebenmal so schweren Krone auf als die herrschenden.

Im Vergleich der herrschenden Bäume ist bei den Eichen der Saat eine ausgewogenere Biomasseverteilung innerhalb der Krone zu beobachten als bei den anderen Beständen. Die Blattmasse ist doppelt so hoch als bei den gepflanzten und verpflanzten Eichen. Die Ästchen haben den geringsten Anteil an der gesamten Kronenmasse. Nach diesen Resultaten haben die Bäume des Saatbestandes starke Äste mit vielen Blättern. Die Eichen des Pflanzungs- und Verpflanzungsbestandes weisen weniger starke Äste auf. Die beherrschten Saateichen besitzen weniger Blätter als die der Pflanzung.

Tabelle 28: Mittlere Probebaumparameter $\boldsymbol{x}$ und Standardabweichungen (sx); Holzminden, 22 jährige Eiche; Saat und Pflanzung

\begin{tabular}{|c|c|c|c|c|}
\hline \multirow[t]{2}{*}{ Fläche } & & Pflanzung & Saat & Verpflanzung \\
\hline & & $x(s x)$ & $\mathbf{x}(s x)$ & $x(s x)$ \\
\hline \multirow[t]{7}{*}{ Kraft 2} & Kronengewicht (kg) & $8,0(2,9)$ & $13,2(4,3)$ & $7,5(3,7)$ \\
\hline & - $\quad$ Ast $(\mathrm{kg})$ & $3,9(1,8)$ & $5,3(2,5)$ & $3,4(1,7)$ \\
\hline & - $\quad$ Ästchen $(\mathrm{kg})$ & $1,9(0,6)$ & $3,4(1,4)$ & $2,0(1,1)$ \\
\hline & - $\quad$ Blatt $(\mathrm{kg})$ & $2,2(0,5)$ & $4,5(0,8)$ & $2,1(1,1)$ \\
\hline & Kronenansatz (m) & $6,9(0,7)$ & $6,4(1,4)$ & $5,2(1,6)$ \\
\hline & Kronenlänge (m) & $4,5(0,6)$ & $5,4(1,3)$ & $5,1(1,2)$ \\
\hline & Kronenprozent (\%) & $39,3(4,4)$ & $46,0(11,3)$ & $49,4(12,2)$ \\
\hline \multirow[t]{7}{*}{ Kraft 4} & Kronengewicht $(\mathrm{kg})$ & $2,1(1,5)$ & $1,9(1,0)$ & \\
\hline & - $\quad$ Ast $(\mathrm{kg})$ & $0,8(0,6)$ & $0,8(0,5)$ & \\
\hline & - $\quad$ Ästchen (kg) & $0,5(0,3)$ & $0,5(0,3)$ & \\
\hline & - $\quad$ Blatt $(\mathrm{kg})$ & $0,8(0,7)$ & $0,6(0,4)$ & \\
\hline & Kronenansatz (m) & $6,9(1,2)$ & $8,0(0,7)$ & \\
\hline & Kronenlänge (m) & $2,8(1,7)$ & $2,7(1,0)$ & \\
\hline & Kronenprozent (\%) & $28,3(14,3)$ & $25,2(8,7)$ & \\
\hline
\end{tabular}

\section{Kattenbühl}

Auch hier fallen Kronengewicht, Kronenprozent und Kronenschirmfläche mit Abnahme des sozialen Status der Eichen ab (Tabelle 29). Das höchste Kronengewicht weist die gepflanzte Eiche mit 13,4 kg auf. Die Krone der Saateiche (Saat I) ist rund $3 \mathrm{~kg}$ leichter, die der Saat II Eiche $(8 \mathrm{~kg})$. Das höchste Kronenprozent besitzt die gepflanzte Eiche mit $70 \%$. Die gesäte Eiche (Saat I) hat relativ eine $6 \%$ und die Saat II Eiche eine $8 \%$ kürzere Krone als die ge- 
pflanzte Eiche. Darüber hinaus überschirmt sie den Boden ein bis zwei Quadratmeter mehr als die anderen Bäume.

Der Anteil der Blätter am Kronengewicht ist bei den herrschenden Eichen am höchsten. Bei den Bäumen der vierten Kraft'schen Klasse ändert sich dieses. Sie zeigen einen höheren Anteil an Ästen. Blätter, Äste und Ästchen stehen insgesamt im Verhältnis $41 \%$ zu $35 \%$ zu $24 \%$.

Tabelle 29: Mittlere Probebaumparameter $\boldsymbol{x}$ und Standardabweichungen (sx); Kattenbühl, 13 jährige Eiche; Saat und Pflanzung

\begin{tabular}{|c|c|c|c|c|}
\hline \multirow[t]{2}{*}{ Fläche } & & Pflanzung & Saat I & Saat II \\
\hline & & $x(s x)$ & $\mathbf{x}(\mathbf{s x})$ & $x(s x)$ \\
\hline \multirow[t]{8}{*}{ Kraft 1} & Kronengewicht $(\mathrm{kg})$ & $13,4(2,8)$ & $10,5(3,5)$ & $5,5(2,1)$ \\
\hline & - Ast (kg) & $4,8(1,0)$ & $3,4(1,8)$ & $1,7(0,7)$ \\
\hline & - Ästchen (kg) & $2,5(1,4)$ & $2,8(1,3)$ & $1,2(0,6)$ \\
\hline & - Blatt (kg) & $6,0(1,5)$ & $4,2(0,7)$ & $2,6(1,4)$ \\
\hline & Kronenansatz (m) & $2,1(0,6)$ & $2,4(0,3)$ & $2,7(0,6)$ \\
\hline & Kronenlänge (m) & $5,0(0,9)$ & $4,5(0,5)$ & $4,4(0,4)$ \\
\hline & Kronenprozent \% & $70,2(9,2)$ & $64,9(4,9)$ & $62,3(6,3)$ \\
\hline & Kronenschirmfläche $\left(\mathrm{m}^{2}\right)$ & $6,6(1,3)$ & $5,7(2,5)$ & $4,3(1,9)$ \\
\hline \multirow[t]{8}{*}{ Kraft 2} & Kronengewicht $(\mathrm{kg})$ & $8,8(3,5)$ & $5,8(2,8)$ & $3,3(1,3)$ \\
\hline & - Ast (kg) & $3,2(1,7)$ & $1,7(0,9)$ & $1,2(0,4)$ \\
\hline & - Ästchen (kg) & $2,1(1,0)$ & $1,5(0,8)$ & $0,7(0,2)$ \\
\hline & - Blatt (kg) & $3,5(1,1)$ & $2,6(1,4)$ & $1,4(0,9)$ \\
\hline & Kronenansatz (m) & $2,1(0,2)$ & $2,2(0,5)$ & $2,3(0,2)$ \\
\hline & Kronenlänge (m) & $4,8(1,0)$ & $3,9(0,8)$ & $3,5(0,5)$ \\
\hline & Kronenprozent \% & $69,6(4,2)$ & $64,0(8,8)$ & $60,3(4,3)$ \\
\hline & Kronenschirmfläche $\left(\mathrm{m}^{2}\right)$ & $4,8(2,1)$ & $4,8(1,4)$ & $3,9(2,2)$ \\
\hline \multirow[t]{8}{*}{ Kraft 3} & Kronengewicht (kg) & $2,4(0,6)$ & $2,1(0,7)$ & $2,8(2,0)$ \\
\hline & - Ast (kg) & $0,7(0,1)$ & $0,5(0,2)$ & $1,1(0,8)$ \\
\hline & - Ästchen (kg) & $0,6(0,2)$ & $0,6(0,2)$ & $0,6(0,4)$ \\
\hline & - Blatt (kg) & $1,2(0,3)$ & $1,0(0,4)$ & $1,1(0,9)$ \\
\hline & Kronenansatz (m) & $2,5(0,4)$ & $2,0(0,6)$ & $2,7(0,7)$ \\
\hline & Kronenlänge (m) & $3,7(0,1)$ & $3,0(0,3)$ & $3,1(0,4)$ \\
\hline & Kronenprozent \% & $60,1(5,0)$ & $59,7(9,3)$ & $53,8(3,6)$ \\
\hline & Kronenschirmfläche $\left(\mathrm{m}^{2}\right)$ & $2,3(0,7)$ & $2,1(0,6)$ & $2,9(1,6)$ \\
\hline \multirow[t]{8}{*}{ Kraft 4} & Kronengewicht (kg) & $1,6(1,7)$ & $0,6(0,1)$ & $0,8(0,5)$ \\
\hline & - Ast (kg) & $0,6(0,7)$ & $0,2(0,0)$ & $0,4(0,3)$ \\
\hline & - Ästchen (kg) & $0,5(0,5)$ & $0,2(0,1)$ & $0,2(0,1)$ \\
\hline & - Blatt (kg) & $0,5(0,4)$ & $0,2(0,0)$ & $0,3(0,2)$ \\
\hline & Kronenansatz (m) & $1,4(0,7)$ & $2,5(0,3)$ & $2,0(0,4)$ \\
\hline & Kronenlänge (m) & $3,0(1,2)$ & $2,1(0,4)$ & $2,3(0,7)$ \\
\hline & Kronenprozent \% & $67,0(18,4)$ & $46,0(7,9)$ & $52,8(13,4)$ \\
\hline & Kronenschirmfläche $\left(\mathrm{m}^{2}\right)$ & $2,0(0,6)$ & $2,5(0,3)$ & $2,8{ }_{(2,8)}$ \\
\hline
\end{tabular}




\subsection{Wurzelparameter}

\subsubsection{Einzelwurzel}

\subsubsection{Wurzeldurchmesserverteilung}

Im folgenden werden die horizontalen und vertikalen Wurzeldurchmesser der ersten Sektion, differenziert nach fünf Klassen, in Kreisdiagrammen dargestellt. Die Größe der Diagramme gibt keinen Aufschluß über absolute Daten. Entsprechende Werte können Anhang II entnommen werden. Für die Fichten, Buchen und Kiefern werden $50 \mathrm{~mm}$ - und für die Eichen $20 \mathrm{~mm}-$ Durchmesserstufen gebildet. Darüber hinaus werden die maximalen Wurzeldurchmesser tabellarisch zusammengefaßt.

\section{Holzerode}

Die Durchmesserverteilung der horizontalen Wurzeln im Buchenreinbestand liegt zwischen $1 \mathrm{~mm}$ und $260 \mathrm{~mm}$. In der Vertikalen sind Wurzeln von $1 \mathrm{~mm}$ bis $190 \mathrm{~mm}$ zu beobachten (Tabelle 30). Sowohl horizontal als auch vertikal befinden sich rund $70 \%$ der Wurzeln in der Klasse $1 \mathrm{~mm}$ bis $50 \mathrm{~mm}$ (Abb. 10). Die Buche im Mischbestand weist horizontal Wurzeln bis $210 \mathrm{~mm}$ und vertikal bis $220 \mathrm{~mm}$ Durchmesser auf. Auch hier sind horizontal und vertikal rund $70 \%$ der Wurzeln dünner als $50 \mathrm{~mm}$.

Die Wurzeldurchmesserverteilung der Fichten unterscheidet sich deutlich von der der Buchen. Im Reinbestand besitzt die Fichte die meisten Horizontalwurzeln $(60 \%)$ in der $1 \mathrm{~mm}$ bis $50 \mathrm{~mm}$ und $100 \mathrm{~mm}$ bis $150 \mathrm{~mm}$-Klasse. Ähnlich wie bei den Buchen befinden sich $70 \%$ der Vertikalwurzeln in der $1 \mathrm{~mm}$ bis $50 \mathrm{~mm}$-Klasse. Insgesamt sind $2 \%$ dicker als $150 \mathrm{~mm}$, dagegen sind es bei der reinen Buche 5\%. Die Fichte im Mischbestand weist horizontal eine gleichmäßige Verteilung der Wurzeldurchmesserklassen auf. Es befinden sich rund $20 \%$ Wurzeln in jeder Durchmesserstufe. Vertikal liegen knapp 50\% in der 1 bis $50 \mathrm{~mm}$-Klasse. $25 \%$ der Wurzeln sind größer als $150 \mathrm{~mm}$, wobei $6 \%$ sogar dicker als $200 \mathrm{~mm}$ sind. Die stärkste Fichtenwurzel erreicht einen Durchmesser von $250 \mathrm{~mm}$ (Tabelle 30) in $30 \mathrm{~cm}$ Entfernung von der Stammbasis.

Tabelle 30: Maximale Durchmesser ${ }_{\text {(wDmax); }}$ der Wurzeln der ersten Sektion; Holzerode; 5565 jährige Buche im Rein- und Mischbestand

\begin{tabular}{|lll|ll|ll|l|rr|}
\hline & \multicolumn{2}{c}{ Buche } & \multicolumn{3}{c}{ Buche / Fichte } & \multicolumn{2}{c|}{ Fichte } & \multicolumn{2}{c|}{ Fichte / Buche } \\
& hor & vert & hor & vert & hor & vert & hor & vert \\
$\mathbf{W D}_{\max }(\mathbf{m m})$ & 260 & 190 & 210 & 220 & 250 & 185 & 245 & 240 \\
\hline
\end{tabular}




\section{Buche horizontal}

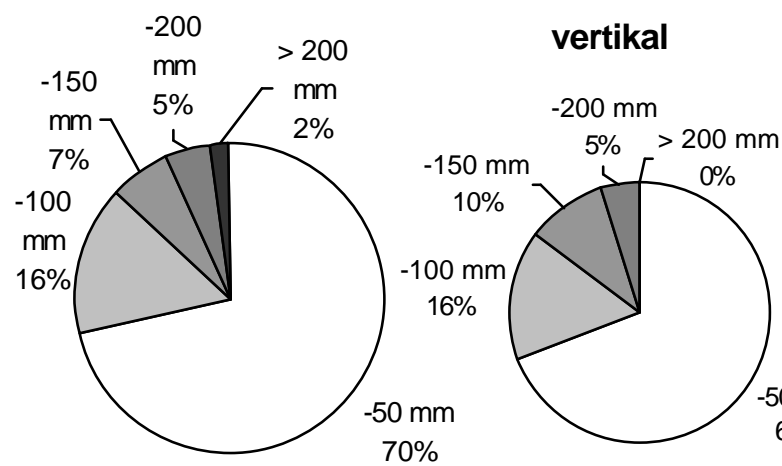

Buche / Fichte horizontal

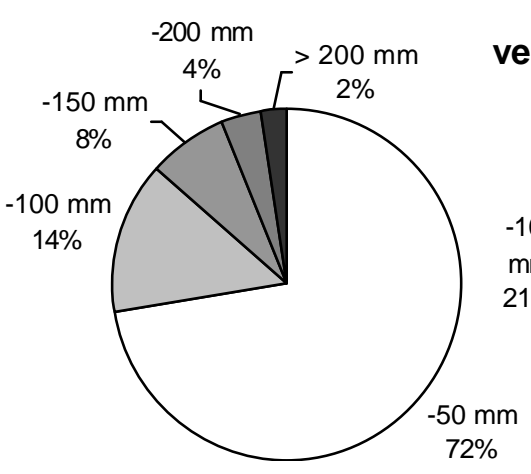

vertikal
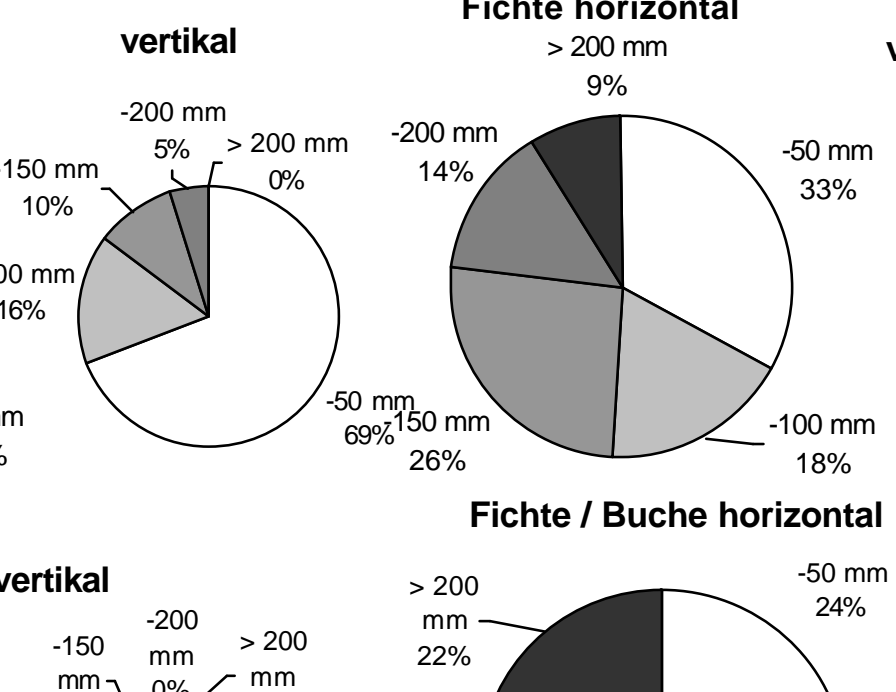

vertikal

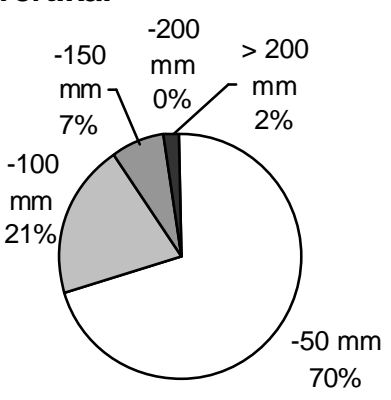

Fichte / Buche horizontal

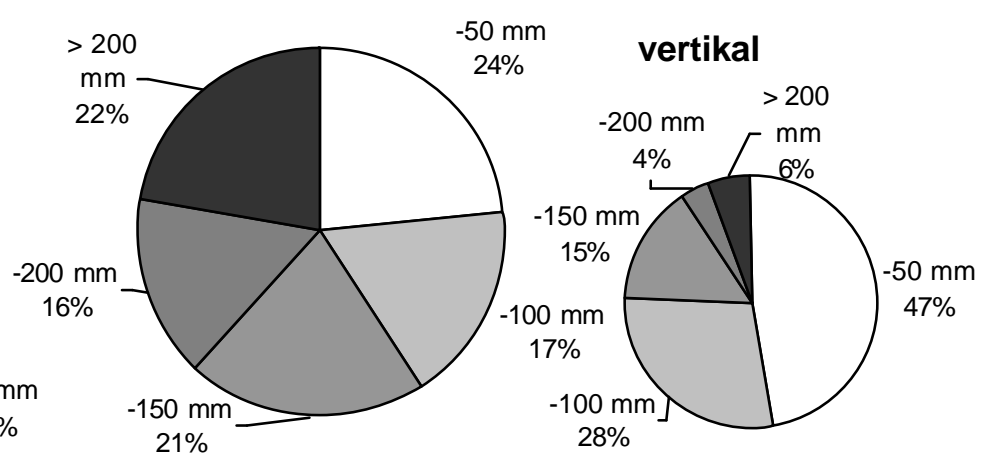

Abb. 10: Wurzeldurchmesserverteilung (50 mm-Klassen) der Horizontal- und Vertikalwurzeln (1. Sektion); Holzerode, 55-68 jährig Buche und Fichte im Rein- und Mischbestand

\section{Krebeck}

Die maximalen Wurzeldurchmesser der untersuchten Fichtenbestände sind Tabelle $31 \mathrm{zu}$ entnehmen. Im Vergleich zum 21 jährigen Bestand steigt der maximale Durchmesser der Horizontalwurzeln im 30jährigen um $75 \%$ auf $210 \mathrm{~mm}$. Bei dem 41 jährigen Bestand erhöht er sich um weitere $30 \%$ auf $270 \mathrm{~mm}$. In vertikaler Richtung erreichen die Wurzeln des 21 jährigen Bestandes maximale Durchmesser von $100 \mathrm{~mm}$. Im 30- und im 41jährigen Bestand liegen die entsprechenden Werte bei $170 \mathrm{~mm}$ bzw. bei $150 \mathrm{~mm}$.

Tabelle 31: Maximale Durchmesser ${ }_{\text {(WDmax); }}$ der Wurzeln der ersten Sektion; Krebeck; 2041 jährige Fichte im Reinbestand

\begin{tabular}{|l|cc|cc|cr|}
\hline Fichte & \multicolumn{2}{c}{$\mathbf{2 1}$ jährig } & \multicolumn{2}{c|}{ 30 jährig } & \multicolumn{2}{c|}{$\mathbf{4 1}$ jährig } \\
Wurzelrichtung & hor & vert & hor & vert & hor & vert \\
WD $_{\max }(\mathrm{mm})$ & 120 & 100 & 210 & 170 & 270 & 150 \\
\hline
\end{tabular}

Abb. 11 zeigt die durchschnittliche Wurzelverteilung der in Krebeck untersuchten Bestände. Die 21 jährigen Fichten werden nicht dargestellt, da dort allein zwei Wurzelklassen zu finden sind. Sie besitzen kaum Wurzeln, die dicker als $100 \mathrm{~mm}$ sind. Dreiviertel aller Horizontalwurzeln befinden sich in der $1 \mathrm{~mm}$ bis $50 \mathrm{~mm}$-Klasse. Das verbleibende Viertel entfällt auf die $50 \mathrm{~mm}$ bis $100 \mathrm{~mm}$-Klasse. Bei dem 30- und dem 41 jährigen Bestand sind rund $40 \%$ der $\mathrm{H}$ rizontalwurzeln über $50 \mathrm{~mm}$ dick. 
Bei der 21 jährigen Fichte sind $10 \%$ der Vertikalwurzeln dicker als $50 \mathrm{~mm}$. Dies trifft bei den 30 jährigen Bäume für $21 \%$ - und bei den 41 jährigen Fichten für $46 \% \mathrm{zu}$.

Fichte 30 horizontal

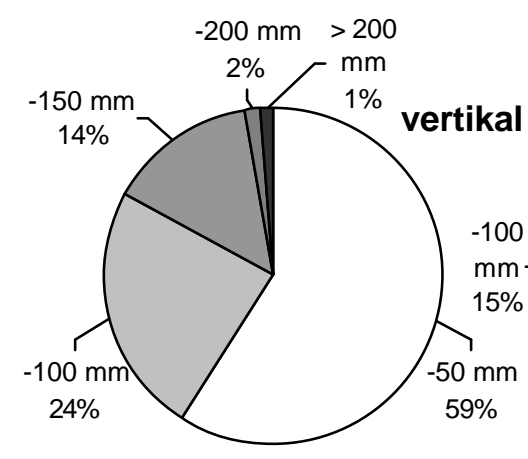

Fichte 41 horizontal

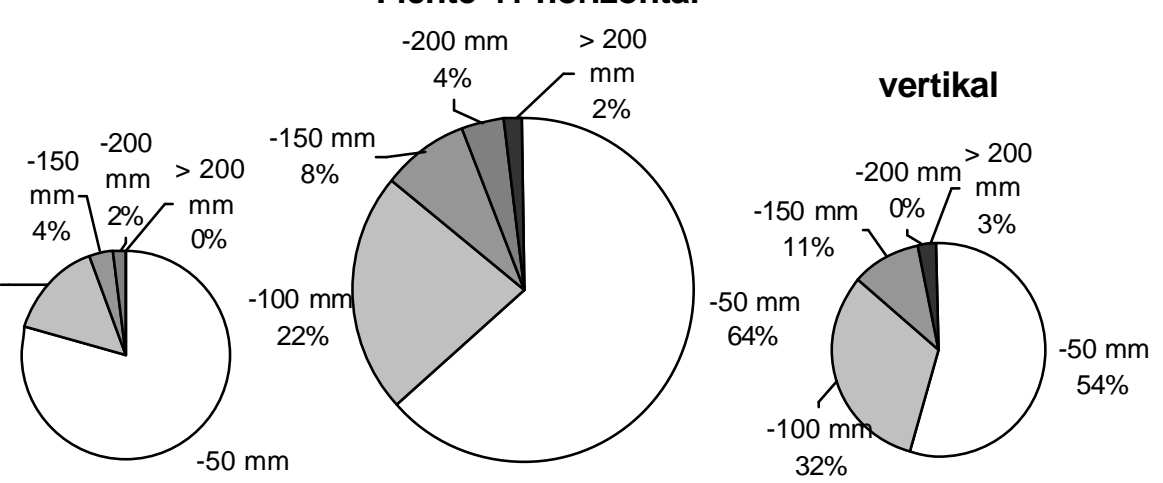

Abb. 11: Wurzeldurchmesserverteilung (50 m-Klassen) der Horizontal- und Vertikalwurzeln (1. Sektion); Krebeck, 30- und 41 jährige Fichte im Reinbestand

\section{Knesebeck}

Die maximalen Wurzeldurchmesser der in Knesebeck untersuchten Bestände sind in Tabelle 32 zusammengestellt. Bei den 20- und dem 31 jährigen Fichten liegen sie horizontal bei $70 \mathrm{~mm}$ bzw. $190 \mathrm{~mm}$. Bei der 50 jährigen Fichten lassen sich maximale Werte von $260 \mathrm{~mm}$ nachweisen.

Tabelle 32: Maximale Durchmesser (WDmax); $_{\text {;er }}$ Wurzeln der ersten Sektion Knesebeck; Fichte und Kiefer, 20 - 50 jährig im Reinbestand

\begin{tabular}{|lcccc|}
\hline \multicolumn{1}{|c}{ WD $_{\max }(\mathbf{m m})$} & \multicolumn{2}{c}{ Fichte } & \multicolumn{2}{c|}{ Kiefer } \\
& horizontal & vertikal & horizontal & vertikal \\
20 jährig & 70 & 40 & 50 & 60 \\
um 30 jährig & 190 & 160 & 150 & 110 \\
41- bzw. 50 jährig & 260 & 170 & 230 & 260 \\
\hline
\end{tabular}

In Abb. 12 sind die Durchmesserverteilungen der in Knesebeck untersuchten Bestände dargestellt. Dabei werden die 20jährigen Fichten nicht berücksichtigt, weil sie horizontal und vertikal allein Wurzeln mit Durchmessern unter $50 \mathrm{~mm}$ besitzen.

Zwei Drittel der Horizontalwurzeln sind bei den 31 jährigen Fichten dünner als $50 \mathrm{~mm}$. Bei den 50 jährigen sind es weniger als die Hälfte. In der vertikalen Richtung besitzt die 31 jährige Fichte $10 \%$ Wurzeln, die dicker als $50 \mathrm{~mm}$ sind, und die 50jährige $50 \%$. 
Fichte 31 horizontal

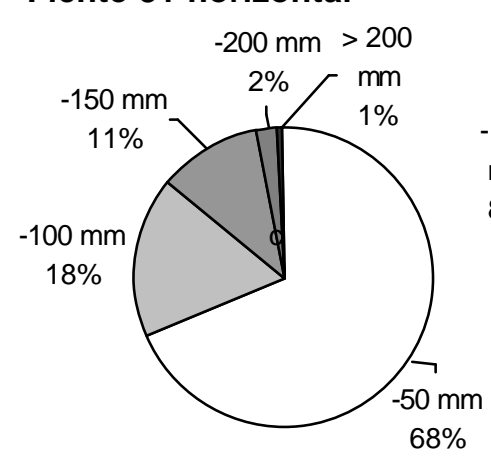

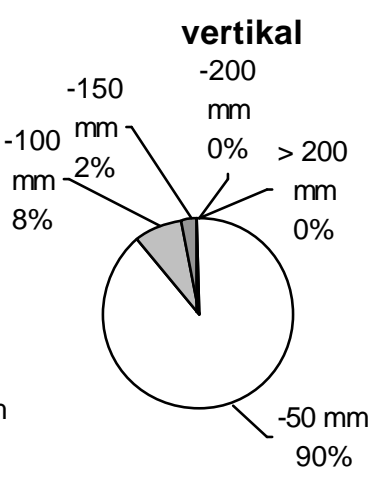

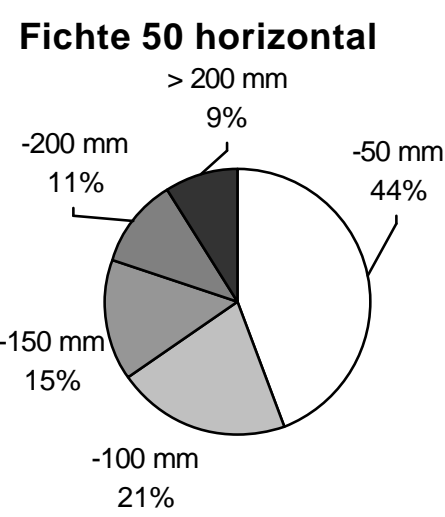

vertikal

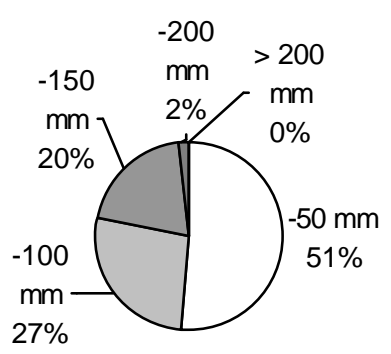

Abb. 12: Wurzeldurchmesserverteilung (50 mm-Klassen) der Horizontal- und Vertikalwurzeln (1. Sektion); Knesebeck, 31 und 50 jährige Fichte im Reinbestand

In Abhängigkeit vom Alter liegen die maximalen Wurzeldurchmesser der untersuchten Kiefernbestände horizontal zwischen $50 \mathrm{~mm}$ und $230 \mathrm{~mm}$. In vertikaler Richtung reichen sie von $60 \mathrm{~mm}$ bis $260 \mathrm{~mm}$ (Tabelle 32).

Die 20jährige Kiefer weist keine Wurzeln auf, die dicker als $50 \mathrm{~mm}$ sind. Aus diesem Grund wurde sie nicht dargestellt. Abb. 13 zeigt, daß die 33jährige Kiefer horizontal nahezu 20\% und vertikal $12 \%$ stärkere Wurzeln als $50 \mathrm{~mm}$ besitzt. Bei den 47 jährigen Bäumen erhöht sich der Prozentsatz der über $50 \mathrm{~mm}$ dicken Wurzeln auf $35 \%$ und vertikal $42 \%$. $17 \%$ der vertikalen Wurzeln sind über $150 \mathrm{~mm}$ dick.

Bei den jungen Beständen läßt sich noch kein Unterschied zwischen Kiefer und Fichte feststellen. Dagegen fällt die Horizontalbewurzelung $<50 \mathrm{~mm}$ der 31 jährigen Fichten gegenüber den etwa gleichalten Kiefer um $14 \%$ höher aus. Dieser Unterschied ist in vertikaler Richtung nicht nachzuweisen. Bei den um 50jährigen Bäumen zeigt die Kiefer horizontal 35\% Wurzeln dicker als $50 \mathrm{~mm}$, die Fichte hingegen schon $56 \%$. Dabei besitzen bei der Kiefer $7 \%$ und bei der Fichte $20 \%$ einen Durchmesser über $150 \mathrm{~mm}$. In der Vertikalen weisen die Kiefer und die Fichte zu $42 \%$ bzw. $49 \%$ dickere Wurzeln als $50 \mathrm{~mm}$ auf. Von diesen sind bei der Fichte die meisten Wurzeln in den Klassen 50 bis $150 \mathrm{~mm}$ zu finden. Dagegen verteilen sich die Wurzeln bei der Kiefer gleichmäßiger auf alle Klassen.
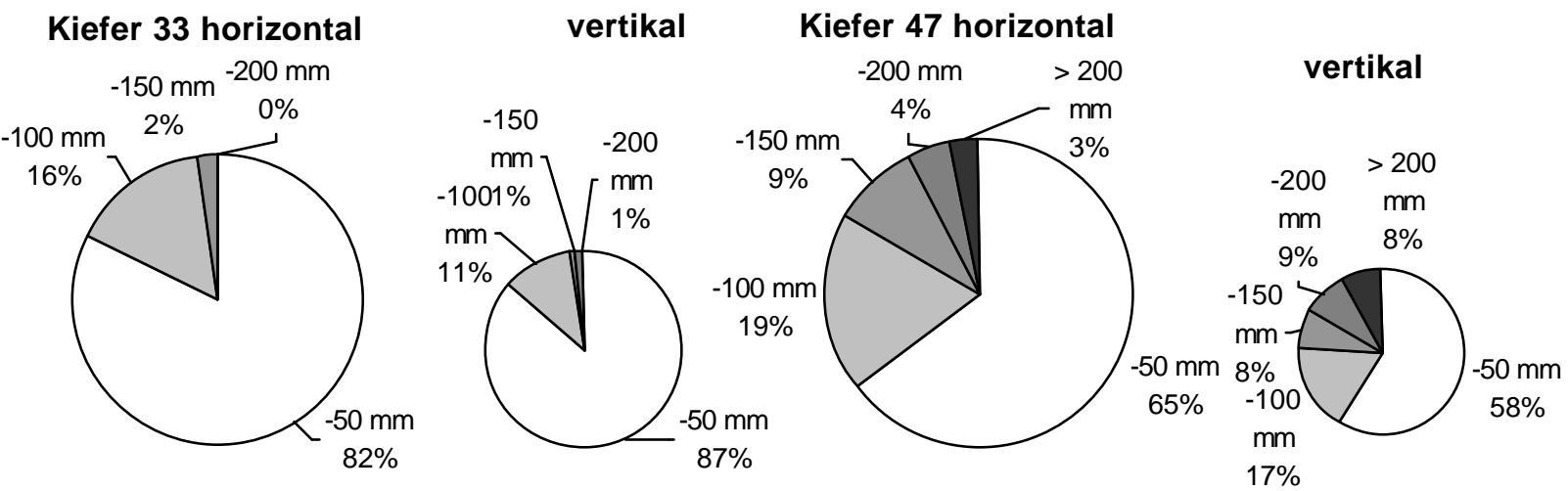

Abb. 13: Wurzeldurchmesserverteilung (50 mm-Klassen) der Horizontal- und Vertikalwurzeln; Knesebeck (1. Sektion); 33- und 47 jährige Kiefer im Reinbestand 


\section{Holzminden}

Die maximalen Durchmesser der Pflanzeichen unterscheiden sich in horizontaler und vertikaler Richtung um 8\% (Tabelle 33). Beim Saatbestand liegen entsprechende Werte zwischen $69 \mathrm{~mm}$ und $143 \mathrm{~mm}$. Die stärkste Wurzel der verpflanzten Eichen hat horizontal einen Durchmesser von $55 \mathrm{~mm}$. Die Vertikalwurzel ist dabei doppelt so stark wie die Horizontalwurzel.

Tabelle 33: Maximale Durchmesser ${ }_{\text {(WDmax); }}$ der Wurzeln der ersten Sektion; Holzminden, 22 jährige Eiche aus Saat und Pflanzung

\begin{tabular}{ccccccc} 
& \multicolumn{2}{c}{ Pflanzung } & \multicolumn{2}{c}{ Saat } & \multicolumn{2}{c}{ Verpflanzung } \\
& horizontal & vertikal & horizontal & vertikal & horizontal & vertikal \\
$\mathbf{W D}_{\max }(\mathrm{mm})$ & 82 & 89 & 69 & 143 & 55 & 92
\end{tabular}

Die Eichen der Pflanzung weisen horizontal $76 \%$ dünne Wurzeln in der $1 \mathrm{~mm}$ bis $20 \mathrm{~mm}$ Klasse auf (Abb. 14). Bei der Saateiche befinden sich $65 \%$ in dieser Klasse, das verbleibende Drittel der Wurzeln ist dicker ist $20 \mathrm{~mm}$. Bei allen Flächen sind horizontal unter $1 \%$ Wurzeln größer $80 \mathrm{~mm}$ zu finden. Auf die Darstellung der verpflanzten Eichen in Abb. 14 wird verzichtet, da die Verteilung in der Horizontalen der der Pflanzeichen und in der vertikalen der der Saateichen ähnelt.

Vertikal besitzt die Eiche der Pflanzung und Verpflanzung rund zur Hälfte Wurzeln größer als $20 \mathrm{~mm}$. Dabei entfallen $43 \%$ auf die Wurzelklassen $20 \mathrm{~mm}$ bis $60 \mathrm{~mm}$. Nur $6 \%$ der Vertikalwurzeln sind in der Klasse von $60 \mathrm{~mm}$ bis $80 \mathrm{~mm}$ zu finden. Die Saateiche weist $41 \%$ dünne Wurzeln (bis $20 \mathrm{~mm}$ ) auf. Die übrigen $60 \%$ sind relativ gleichmäßig auf die vier größeren Wurzelklassen verteilt.

Pflanzung horizontal

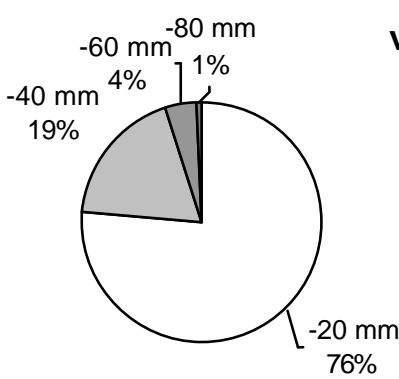

vertikal

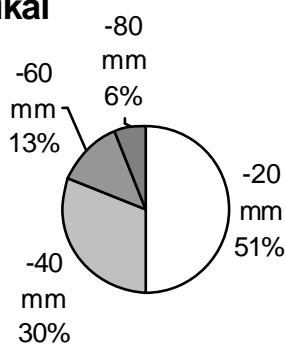

Saat horizontal

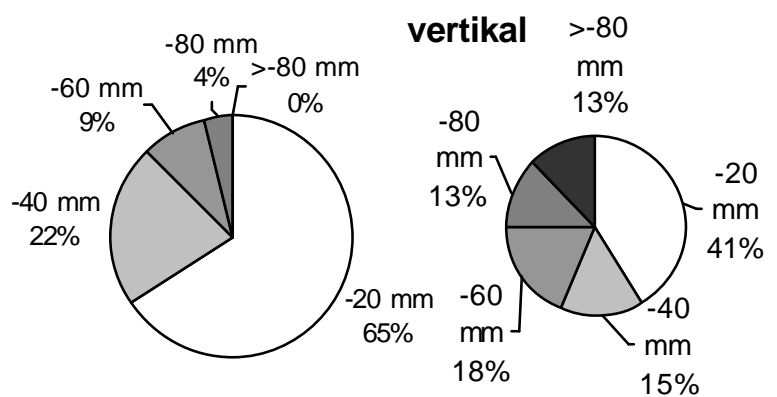

Abb. 14: Wurzeldurchmesserverteilung (20 mm-Klassen) der Horizontal- und Vertikalwurzeln (1. Sektion); Holzminden, 22 jährige Eiche aus Saat und Pflanzung 


\section{Kattenbühl}

Die maximalen Wurzeldurchmesser der in Kattenbühl untersuchten Eichen reichen horizontal bis $63 \mathrm{~mm}$ und vertikal bis $67 \mathrm{~mm}$. Dabei weisen die Eichen der Saat I Fläche die stärksten Wurzeln auf.

Tabelle 34: Maximale Durchmesser ${ }_{\text {(WDmax); }}$;er Wurzeln der ersten Sektion; Kattenbühl, Eiche 13 jährig aus Saat und Pflanzung

\begin{tabular}{|ccccccc|}
\hline & \multicolumn{2}{c}{ Pflanzung } & \multicolumn{2}{c|}{ Saat I } & \multicolumn{2}{c|}{ Saat II } \\
& horizontal & vertikal & horizontal & vertikal & horizontal & vertikal \\
$\mathbf{W D}_{\max }(\mathrm{mm})$ & 63 & 42 & 62 & 67 & 53 & 50 \\
\hline
\end{tabular}

Die drei Bestände unterscheiden sich dadurch, daß in der Horizontalen die gepflanzten Eichen rund 20\% Wurzeln besitzen, die einen größeren Durchmesser als $20 \mathrm{~mm}$ haben. Bei den Eichen des Saat I Bestandes sind es 10\% und bei denen des Saat II Bestandes 6\% (Abb. 15).

Im Schnitt besitzen sowohl die gepflanzten Eichen als auch die gesäten Eichen des Saat II Bestandes $17 \%$ Vertikalwurzeln, die dicker als $20 \mathrm{~mm}$ sind. Dabei weisen Erstere keine Vertikalwurzeln über $40 \mathrm{~mm}$ auf, Letztere besitzen hingegen 3\%. Bei der gesäten Eiche (Saat I Fläche) sind $34 \%$ stärkere Wurzeln als $20 \mathrm{~mm} \mathrm{zu}$ beobachten, wobei $6 \%$ sich in der $40 \mathrm{~mm}$ $60 \mathrm{~mm}$-Klasse befinden.
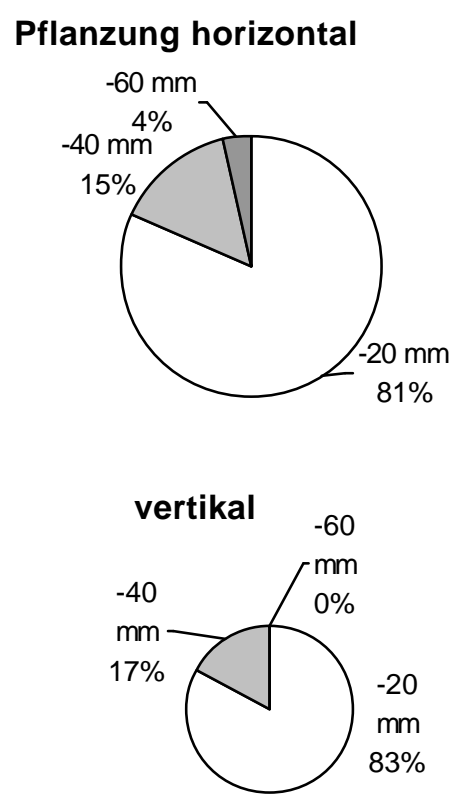

\section{Saat I horizontal}

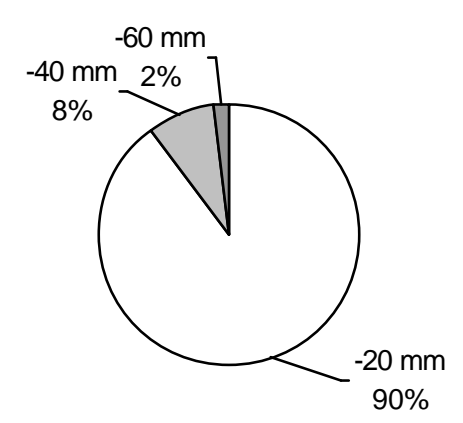

vertikal

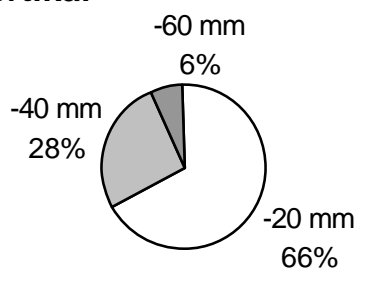

Saat II horizontal

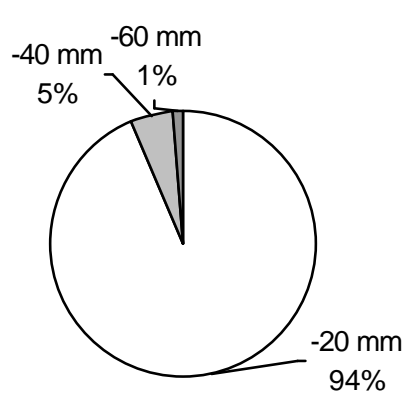

vertikal $\quad-60 \mathrm{~mm}$

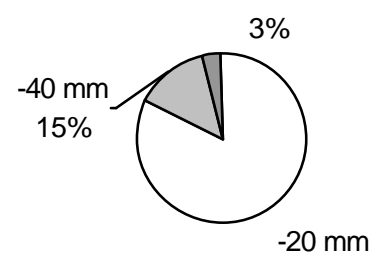

$82 \%$

Abb. 15: Wurzeldurchmesserverteilung (20 mm-Klassen) der Horizontal- und Vertikalwurzeln; Kattenbühl (1. Sektion); 13 jährige Eiche aus Saat und Pflanzung 


\subsubsection{Wurzelsystem}

\subsubsection{Tiefendurchwurzelung}

Die Abbildungen 16 und 17 stellen die durchschnittliche und maximale Tiefe der untersuchten Wurzeln der Fichte, Buche, Kiefer und Eiche dar.

\section{Holzerode}

Durchschnittlich wurzeln die Mischbestandsfichten $18 \%$ (signifikant) bzw. $14 \%$ tiefer als die Fichten des Reinbestandes und die Buchen des Reinbestandes (Abb. 16). Am flachsten wurzelt die Buche des Mischbestandes. Ihre Wurzeln erreichen eine durchschnittliche Tiefe von etwa $60 \mathrm{~cm}$. Die Wurzeln der Fichten und Buchen der anderen Flächen erreichen dabei Tiefen von $70 \mathrm{~cm}$ bis $82 \mathrm{~cm}$. Die maximal tiefsten Wurzeln sind rund $40 \mathrm{~cm}$ tiefer im Boden zu finden.

\section{Krebeck}

Die durchschnittliche Tiefe der Fichtenwurzeln steigt vom 21- zum 30 jährigen um $41 \%$ (signifikant) und vom 30- zum 41 jährigen Bestand um 19\%. Dabei wurzeln die Fichten etwa zwischen $40 \mathrm{~cm}$ und $70 \mathrm{~cm}$ tief. Die tiefste Wurzel liegt bei allen Beständen rund $40 \mathrm{~cm}$ tiefer als die mittlere Wurzel. Die maximale Wurzeltiefe der Fichten liegt im Schnitt beim 30 jährigen Bestand $25 \%$ (signifikant) tiefer als im jungen Bestand. Gegenüber den 41 jährigen- wurzeln die 30 jährigen Fichten $6 \%$ flacher. Die Variationskoeffizienten der mittleren und der maximalen Wurzeltiefe liegen zwischen $20 \%$ und $30 \%$. Je jünger der Bestand, desto größer ist die Streuung der beiden berechneten Tiefenwerte.

\section{Knesebeck}

Im Schnitt liegen die Wurzeln der untersuchten Fichten in einer Tiefe von $30 \mathrm{~cm}$ bis $68 \mathrm{~cm}$. Maximal erreichen sie eine Tiefe von $63 \mathrm{~cm}$ bis $119 \mathrm{~cm}$. Durchschnittlich wurzeln die 20 jährigen Fichten um $53 \%$ (signifikant) flacher als die 31 jährigen. Letztere wurzeln $13 \%$ flacher als die 50 jährigen Bäume. Die tiefste Wurzel liegt bei allen untersuchten Fichten rund $40 \mathrm{~cm}$ tiefer als die durchschnittliche Wurzel. Dabei wurzeln die Fichten des jungen Bestandes um $36 \%$ flacher als die des 31 jährigen Bestandes. Die Bäume der letztgenannten Fläche wurzeln $11 \%$ flacher als die der 50 jährigen Fläche. Die Variationskoeffizienten der durchschnittlichen und der maximalen Wurzeltiefe liegen bei allen Beständen zwischen $20 \%$ und $40 \%$. Der junge Bestand besitzt ebenso wie der in Krebeck die größte Streuung der Werte.

Bei den in Knesebeck untersuchten Kiefern nimmt die durchschnittliche Wurzeltiefe vom jüngsten bis zum ältesten Bestand um mehr als das Doppelte zu. Sie beträgt bei den 20 jährigen Kiefern $32 \mathrm{~cm}$ und bei den 47 jährigen $68 \mathrm{~cm}$. Maximal wurzeln die Kiefern zwischen 40 und $50 \mathrm{~cm}$ tiefer als durchschnittlich. Dabei wurzeln die 20 jährigen Kiefern $16 \%$ (signifikant) flacher als die 33 jährigen. Letztgenannte Gruppe wurzelt im Schnitt $25 \%$ (signifikant) flacher als die Bäume des 47 jährigen Bestandes. 


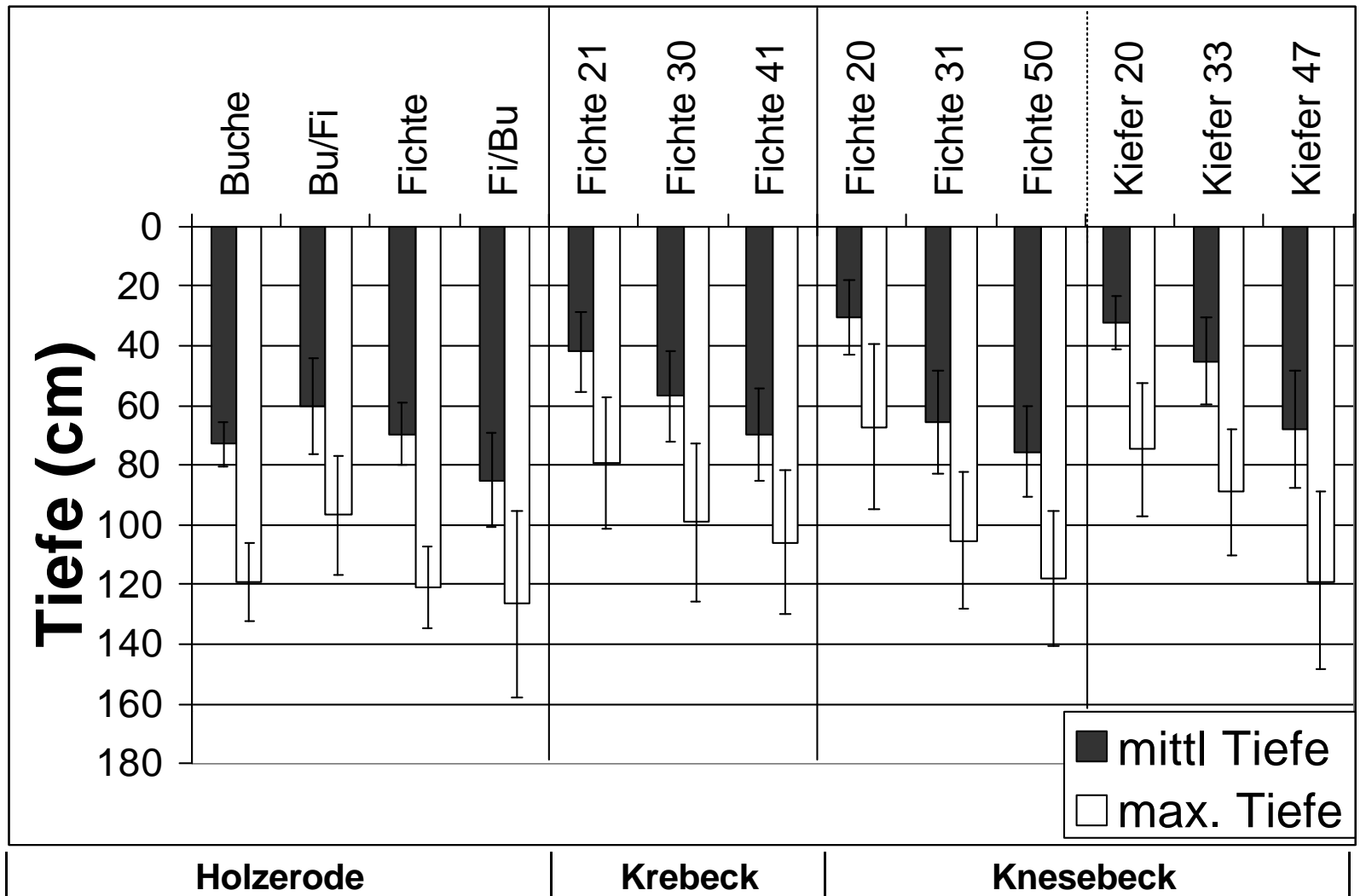

Abb. 16: Darstellung der mittleren und maximalen Gesamttiefe und Standardabweichung; Holzerode (Fichte und Buche, im Mittel 60 jährig im Misch- und Reinbestand), Krebeck (Fichte 20-41 jährig, Reinbestand) und Knesebeck (Fichte und Kiefer 2050 jährig, Reinbestand)

\section{Holzminden}

Bei den untersuchten Eichen sind keine signifikanten Unterschiede in der maximalen Wurzeltiefe (Abb. 17) zu erkennen. Maximal wurzelt eine Saateiche rund $80 \mathrm{~cm}$ tief und damit $10 \mathrm{~cm}$ tiefer als die gepflanzte oder verpflanzte Eiche. Dagegen unterscheiden sich die mittleren Wurzeltiefen der drei Bestände nur geringfügig. Im Schnitt wurzeln die Saateichen $3 \mathrm{~cm}$ tiefer als die gepflanzten und verpflanzten Eichen. Allerdings kann dieser Unterschied statistisch nicht abgesichert werden. Bei Differenzierung der Saat- und der Pflanzeichen nach sozialer Stellung ist ein signifikanter Unterschied zwischen den Kraft'schen Klassen 2 und 4 festzustellen.

\section{Kattenbühl}

Die Tiefendurchwurzelung der Bestände in Kattenbühl wird horizontal und vertikal getrennt ermittelt. Diese Vorgehensweise dient der Prüfung, ob eine Horizontalwurzel mit einem Winkel von zum Beispiel $40^{\circ}$ die gleiche Tiefe erreichen kann wie eine Vertikalwurzel. In Abb. 17 ist zu erkennen, daß eine durchschnittliche horizontale Einzelwurzel signifikant flacher wurzelt als eine Vertikalwurzel (rund $50 \%$ ). Die vertikalen Wurzeln reichen bis $48 \mathrm{~cm}$, die horizontalen bis $23 \mathrm{~cm}$ tief in den Boden. Der Unterschied in der mittleren Durchwurzelungstiefe der horizontalen und vertikalen Wurzeln kann damit bestätigt werden. Die Variationskoeffizienten betragen für die horizontalen Wurzeln der Pflanzung 19\%, für die der Saat I $24 \%$ und die der Saat II $28 \%$. Vertikal liegen sie zwischen $14 \%$ und $17 \%$. 
Die maximale Tiefe der Horizontal- und Vertikalwurzeln der Eichen aller Bestände differiert nicht stark. Die Vertikalwurzeln reichen bei den Eichen auf der Saat I und der Saat II Fläche rund $12 \%$ tiefer in den Boden als die tiefsten Horizontalwurzeln. Diesbezüglich läßt sich bei den gepflanzten Eichen so gut wie keine Differenz feststellen. Für die maximal tiefsten Wurzeln liegen die Variationskoeffizienten zwischen $20 \%$ und $35 \%$. Für die maximale Tiefenreichweite ist keine signifikante Differenz zwischen Horizontal- und Vertikalwurzeln festzustellen.

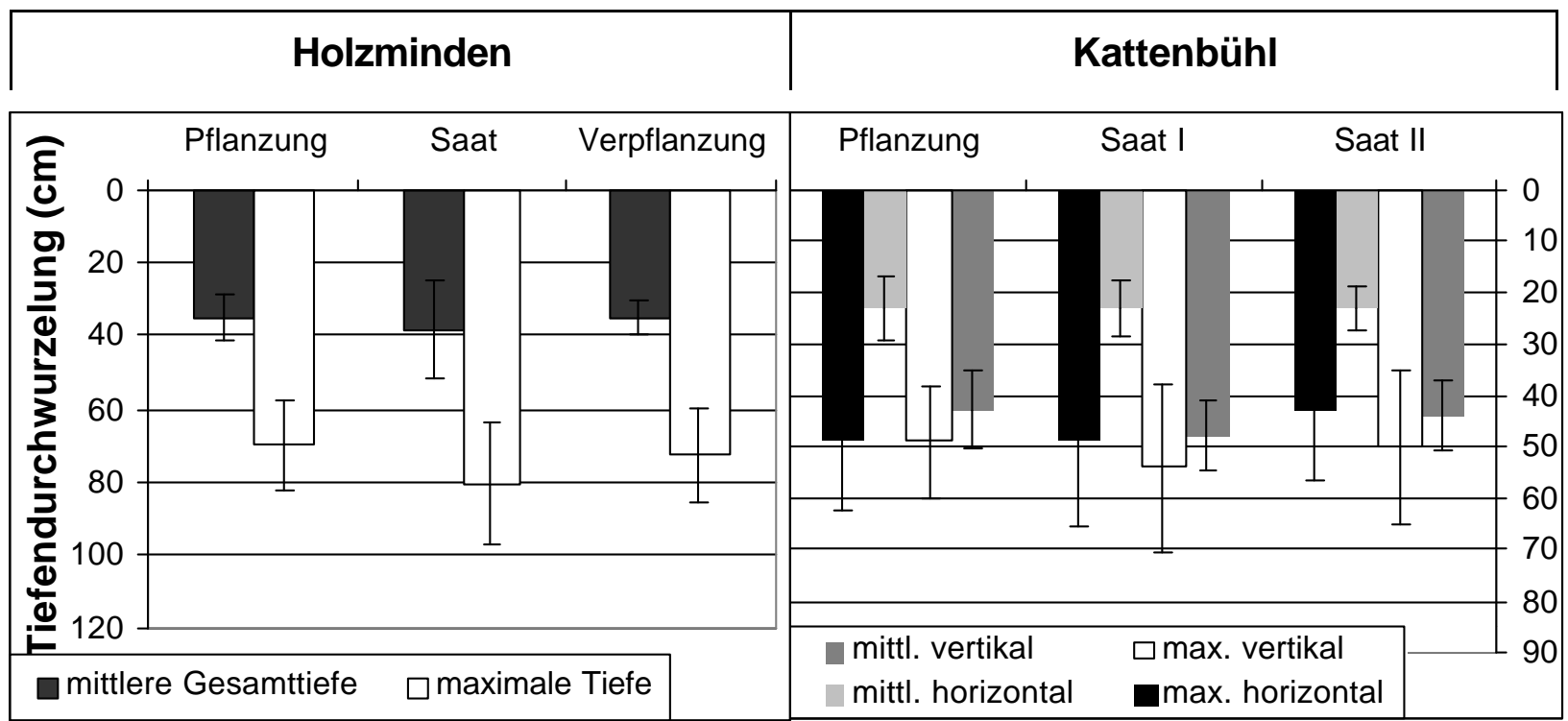

Abb. 17: Darstellung der mittleren und maximalen Gesamttiefe und Standardabweichung der 14- und 22 jährigen Eichen, Holzminden und Kattenbühl

\subsubsection{Querschnittfläche und Wurzelanzahl der horizontalen und vertikalen Wurzeln (Sektion 1)}

Um ein Bild über die Wurzelverteilung zu bekommen, werden im folgenden die Parameter "Wurzelanzahl" und "Wurzelquerschnittfläche" dargestellt. Beide Größen werden für die untersuchten Sektionen berechnet. Zunächst wird die erste Sektion durch die Auswertung der absoluten Meßwerte erörtert. Danach folgt eine Verhältnisbetrachtung der zweiten und dritten Sektion in den sich anschließenden Abschnitten.

\section{Holzerode}

In Abb. 18 wird für einen durchschnittlichen Baum eines Misch- oder Reinbestandes die Summe der Wurzelquerschnittfläche $\left(\mathrm{cm}^{2}\right)$ dargestellt. Horizontal erreichen die Buchenwurzeln im Mittel der Bestände mit 16 Wurzeln eine Querschnittfläche von rund $500 \mathrm{~cm}^{2}$. Die Fichten besitzen bei nur 10 Wurzeln eine Querschnittfläche von über $1000 \mathrm{~cm}^{2}$. Sie weisen in horizontaler Richtung signifikant höhere Querschnittflächen auf als die Buchen.

Beim Vergleich der Bestandesformen zeigt die Buche im Mischbestand an Querschnittflächensumme durchschnittlich $8 \%$ mehr in der Horizontalen $\left(43 \mathrm{~cm}^{2}\right)$ und $46 \%$ weniger in der Vertikalen $\left(174 \mathrm{~cm}^{2}\right)$ als im Reinbestand. Die Fichte besitzt im Schnitt im Mischbestand horizontal eine $11 \%$ und vertikal eine viermal so hohe Querschnittfläche als im Reinbestand. Sie entwickelt in Mischung mit Buche höhere Querschnittflächensummen der Horizontal- und Vertikal- 
wurzeln (signifikant), während die Buche in Mischung mit Fichte geringere in der Vertikalen und höhere Wurzelquerschnitte in der Horizontalen aufweist.

Im folgenden wird die Anzahl der Wurzeln dargestellt, die eine Fichte oder Buche in der ersten Sektion besitzt (Abb. 18). Von allen in Holzerode untersuchten Beständen weist die Buche im Mischbestand horizontal die größte Wurzelanzahl auf. Dabei zeigt sie signifikant mehr Wurzeln als im Reinbestand. Ebenso weist die Fichte im Mischbestand mit 8 Wurzeln horizontal eine signifikant geringere Anzahl auf als im Reinbestand.

Vertikal besitzt die Buche im Reinbestand mit 12 Wurzeln die größte Anzahl. Sie zeigt signifikant $(50 \%)$ mehr Wurzeln als die Buche im Mischbestand. Die Fichten weisen in der vertikalen Wurzelrichtung mehr als halb soviele Wurzeln auf wie die Buchen.

Zusammenfassend läßt sich feststellen, daß die Fichte mit wenig Wurzeln eine größere Querschnittfläche erreicht als die Buche. Die Buche zeigt im Mischbestand vertikal signifikant geringere Querschnittsflächen bei signifikant weniger Wurzeln als im Reinbestand. Die Fichte hingegen weist im Mischbestand eine signifikant größere Querschnittfläche auf. Die Variationskoeffizienten liegen bei den horizontalen Wurzeln zwischen $20 \%$ und $50 \%$, während sie bei den vertikalen auf über $50 \%$ ansteigen.

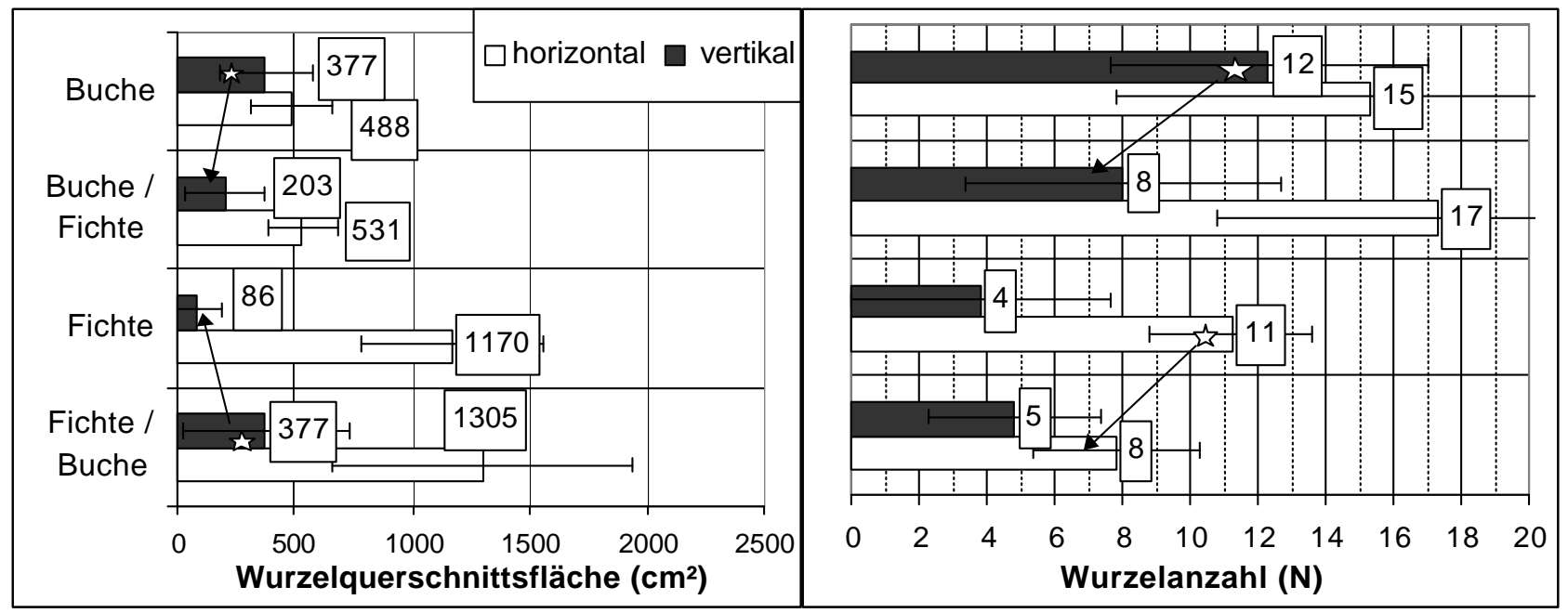

Abb. 18: Mittlere Summe der Wurzelquerschnittfläche und Wurzelanzahl pro Baum und Standardabweichung (1. Sektion); Holzerode, Fichte und Buche (is signifikant $\longrightarrow$ zu)

\section{Krebeck}

Die Absolutwerte der Wurzelquerschnittflächensumme je Fichte bewegen sich in Abhängigkeit vom Alter horizontal zwischen etwa $150 \mathrm{~cm}^{2}$ und $750 \mathrm{~cm}^{2}$ und vertikal zwischen $30 \mathrm{~cm}^{2}$ und $100 \mathrm{~cm}^{2}$ (Abb. 19). Die Variationskoeffizienten der Wurzelquerschnittflächen liegen bei allen drei Flächen über $60 \%$. Die mittlere Wurzelquerschnittflächensumme der horizontalen Fichtenwurzeln steigt in der ersten Sektion signifikant von Bestand zu Bestand auf das Doppelte an. Vertikal erhöht sie sich von 21 zu 30 Jahren auf mehr als das Doppelte und von 30 zu 41 Jahren um die Hälfte.

In dem 21 jährigen Fichtenbestand besitzt jeder Baum durchschnittlich 13 Horizontalwurzeln (Abb. 19). Die 30 jährigen Fichten haben $17 \%$-, die 41 jährigen $32 \%$ weniger Wurzeln. Verti- 
kal weisen die Bäume des 21- und des 30 jährigen Bestandes durchschnittlich vier und die des 41 jährigen zwei Wurzeln auf. Letztere besitzten damit horizontal signifikant weniger Wurzeln als die jüngeren Flächen. Die Variationskoeffizienten der Wurzelanzahl liegen in horizontaler Richtung zwischen $18 \%$ und $29 \%$ und in vertikaler Richtung zwischen 60 und $65 \%$.

Zusammenfassend kann gesagt werden, daß mit zunehmendem Alter die Wurzelquerschnittfläche steigt und die Wurzelanzahl abfällt.

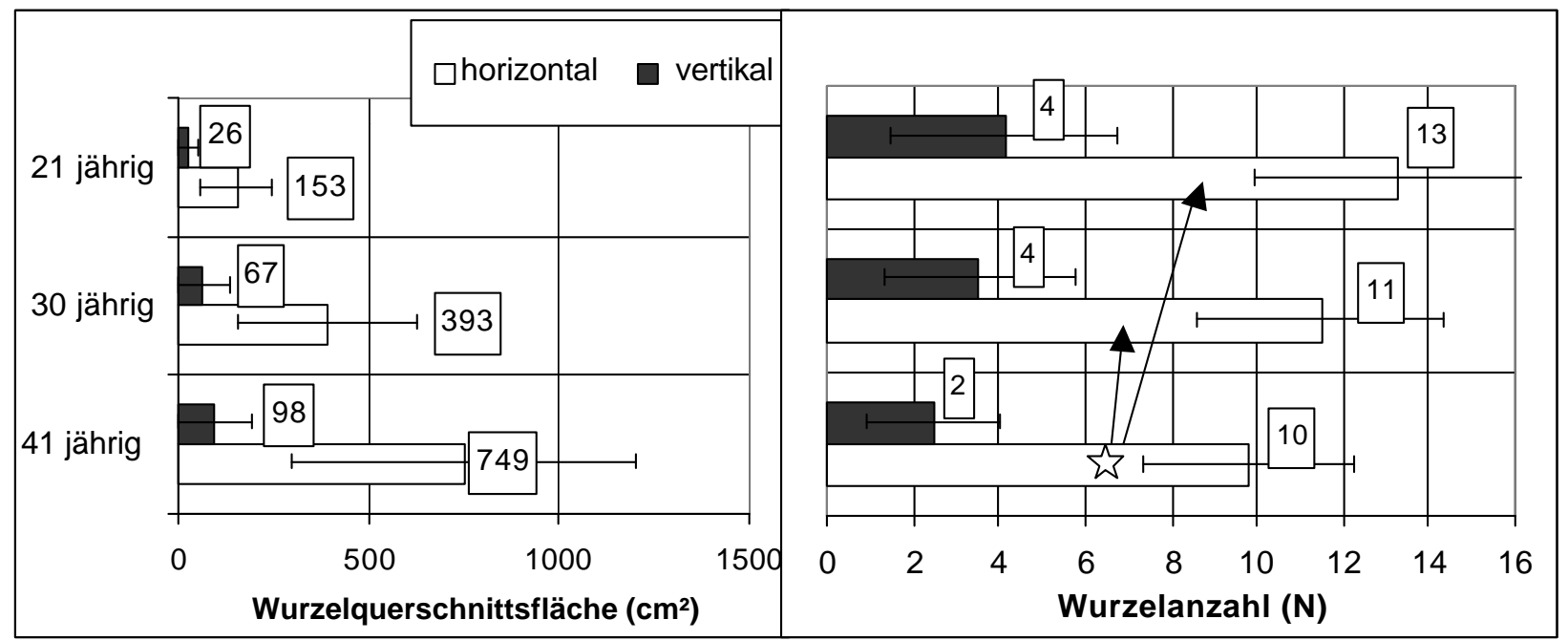

Abb. 19: Mittlere Summe der Wurzelquerschnittfläche und Wurzelanzahl pro Baum und Standardabweichung (1. Sektion); Krebeck, Fichte (ఓ̌signifikant $\longrightarrow z u$ )

\section{Knesebeck}

Die Abbildungen unten (Abb. 20, Abb. 21) stellen die Wurzelanzahl und die Summe der Querschnittsflächen der Fichte und Kiefer des Untersuchungsgebietes Knesebeck dar.

Die jüngsten Fichten weisen horizontal eine Wurzelquerschnittflächensumme von durchschnittlich $40 \mathrm{~cm}^{2}$ auf. In vertikaler Richtung liegt der entsprechende Wert bei $7 \mathrm{~cm}^{2}$. Im Schnitt beträgt die Summe der Wurzelquerschnittflächen der ältesten Fichten horizontal $989 \mathrm{~cm}^{2}$ und vertikal $136 \mathrm{~cm}^{2}$. Diese Werte sind in horizontaler und vertikaler Richtung gegenüber den jüngeren Beständen signifikant höher. Mit Zunahme des Alters steigt die Wurzelquerschnittfläche der vertikalen Wurzeln von $20 \mathrm{zu} 31$ Jahren um das siebenfache und von $31 \mathrm{zu} 50$ Jahren auf das Zweieinhalbfache.

Alle Fichten besitzen in der Horizontalen 11 Wurzeln (Abb. 20). Vertikal schwankt die Wurzelanzahl in Abhängigkeit vom Alter der Bäume zwischen drei (20 jährig) und fünf (50 jährig). 


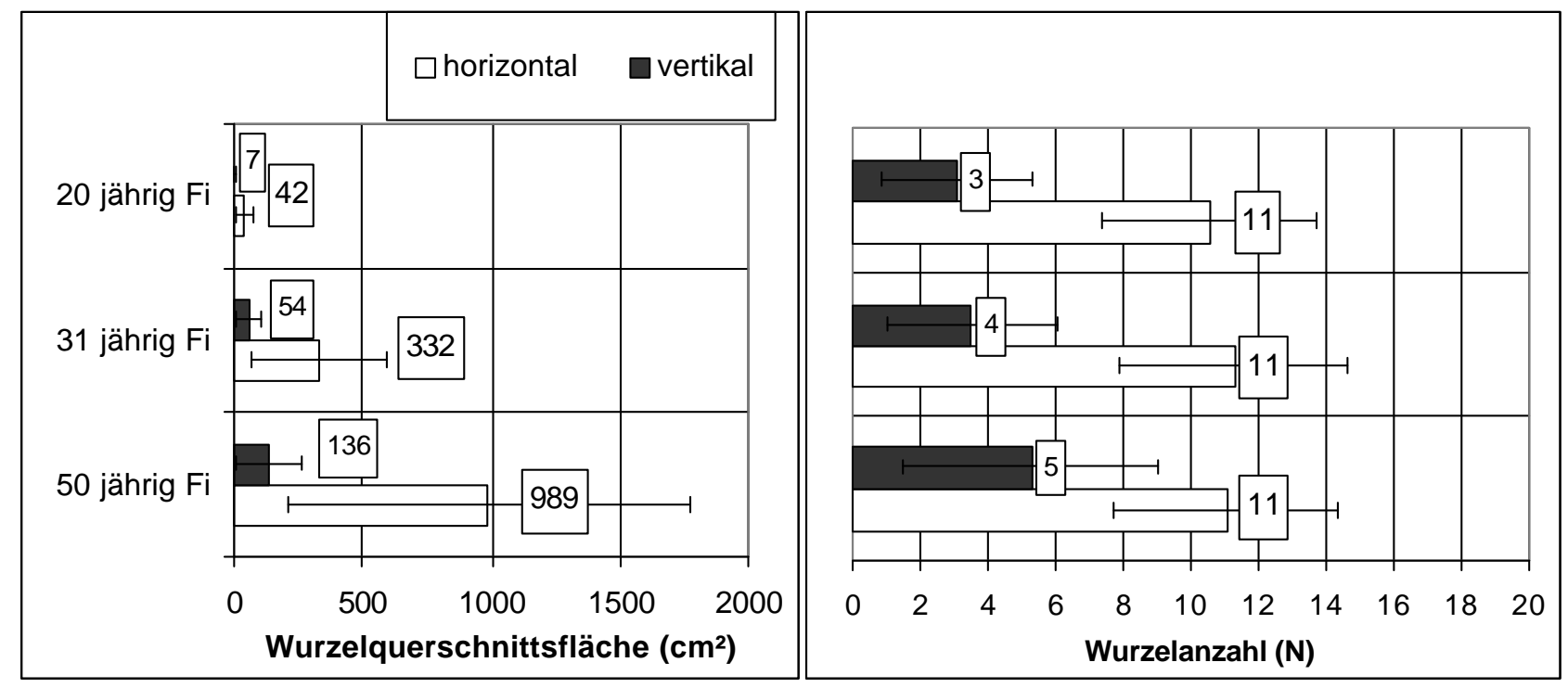

Abb. 20: Mittlere Summe der Wurzelquerschnittfläche und Wurzelanzahl pro Baum und Standardabweichung (1. Sektion); Knesebeck, Fichte

Bei der Kiefer steigt im Schnitt die Wurzelquerschnittfläche pro Baum horizontal von der 20 jährigen zur 33 jährigen auf das Zwölffache und von der 33- zur 47 jährigen Kiefer auf das Vierfache an (Abb. 21). Die jüngeren Bäume besitzen absolut eine Wurzelquerschnittflächensumme von $14 \mathrm{~cm}^{2}$, die 33 jährigen eine von $162 \mathrm{~cm}^{2}$ und die ältesten eine von $642 \mathrm{~cm}^{2}$. In vertikaler Richtung erhöhen sich die Werte von 20- auf 33 jährig auf das Vierfache und von 33 auf 47 jährig auf das Fünffache. Dabei haben die jungen Kiefern eine Wurzelquerschnittfläche von $14 \mathrm{~cm}^{2}$ und die ältesten eine von $292 \mathrm{~cm}^{2}$.

Die durchschnittliche Wurzelanzahl pro Baum steigt von dem 20- zum 47 jährigen Bestand horizontal und vertikal auf das Doppelte an (Abb. 21). Bei den beiden älteren Flächen ist keine Veränderung der Wurzelanzahl festzustellen. Sie besitzen in der Horizontalen 15 Wurzeln und in der Vertikalen vier Wurzeln.

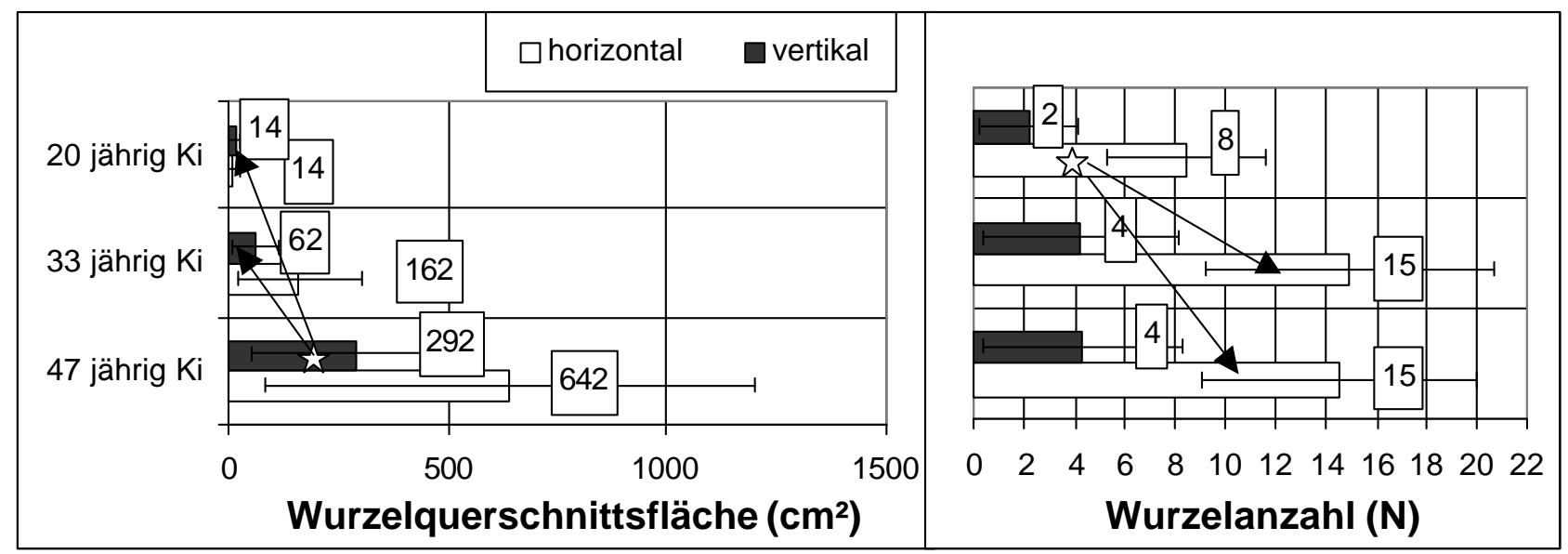

Abb. 21: Summe der Wurzelquerschnittfläche und Wurzelanzahl pro Baum und Standardabweichung (1. Sektion); Knesebeck, Kiefer (§isignifikant $\longrightarrow z u$ ) 


\section{Holzminden}

Die 22 jährigen Eichen zeigen in der ersten Sektion in vertikaler Richtung ähnlich hohe Wurzelquerschnittflächen wie in horizontaler (Abb. 22). Die Summe der vertikalen Wurzelquerschnittflächen liegt, abhängig vom Bestand, zwischen $43 \mathrm{~cm}^{2}$ und $86 \mathrm{~cm}^{2}$. Sie wird durch vier bis acht Wurzeln pro Baum gebildet. Horizontal sind daran 15 bis 19 Wurzeln mit einer Querschnittfläche von $60 \mathrm{~cm}^{2}$ bis $72 \mathrm{~cm}^{2}$ beteiligt. Beim Vergleich der drei Bestände weist die Saateiche horizontal bei $21 \%$ weniger Wurzeln eine rund $18 \%$ größere Wurzelquerschnittfläche auf als die Eiche der Pflanzung und die der Verpflanzung.

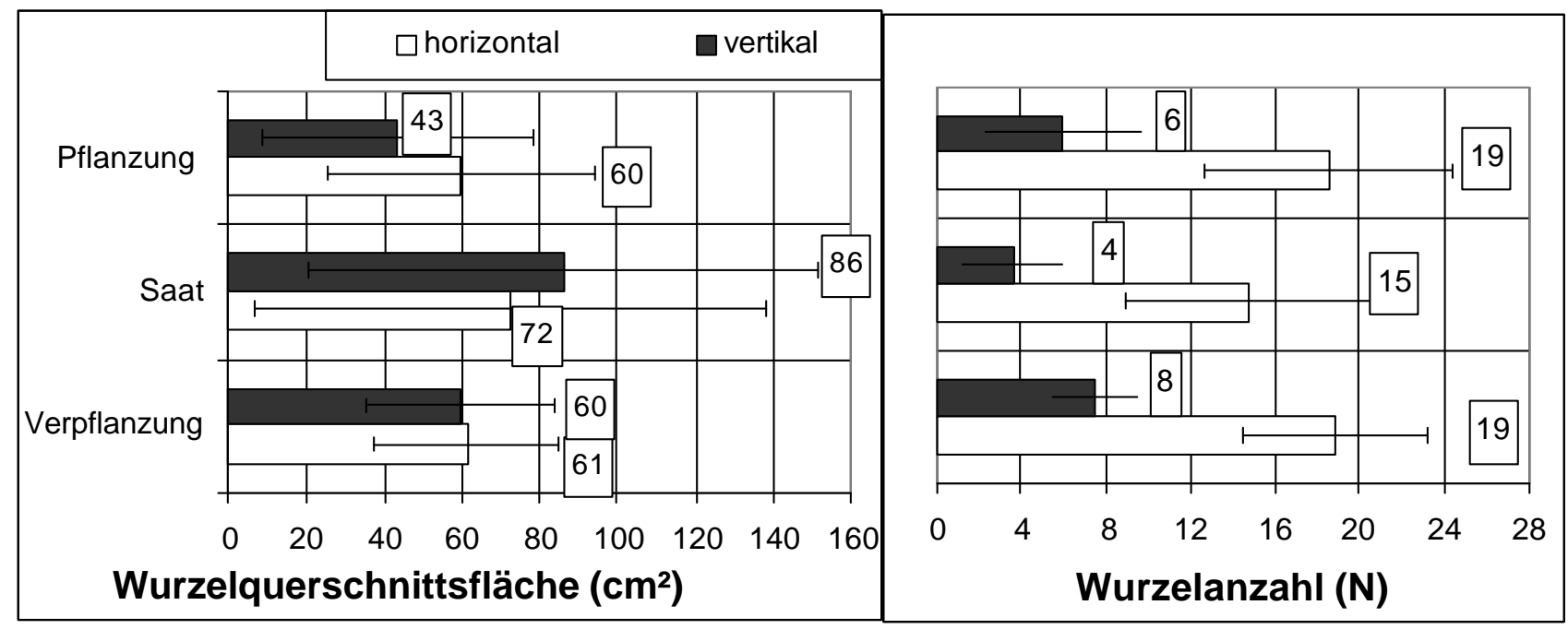

Abb. 22: Summe der Wurzelquerschnittfläche und Wurzelanzahl pro Baum und Standardabweichung (1. Sektion); Holzminden, 22 jährige Eiche

Da bei den verpflanzten Eichen nur herrschende Bäume entnommen wurden, wird nachfolgend eine Differenzierung nach sozialer Stellung vorgenommen. Dies wird in Tabelle 35 dargestellt. Weiterhin wird bei diesem Bestand die Bedeutung der Pfahlwurzel in Abhängigkeit von der sozialen Stellung erörtert. Dabei sind die Werte der Pfahlwurzelquerschnittflächen ein Bestandteil der entsprechenden vertikalen Daten.

Tabelle 35: Summe der Wurzelquerschnittläche und Wurzelanzahl pro 22 jährige Eiche, differenziert nach Kraft; Holzminden ( ${ }^{*}$ signifikant unterschiedlich $\longrightarrow$ zu)

\begin{tabular}{|c|c|c|c|c|c|c|}
\hline \multirow{5}{*}{$\begin{array}{l}\text { Pflanzung } \\
\text { Saat }\end{array}$} & \multicolumn{6}{|c|}{ Wurzelquerschnittfläche $\left(\mathrm{cm}^{2}\right)$} \\
\hline & \multirow{2}{*}{$\begin{array}{c}\text { Kraft } 2 \\
\text { horizontal }\end{array}$} & \multirow{3}{*}{$\begin{array}{c}\text { vertikal } \\
56,7\end{array}$} & \multicolumn{3}{|c|}{ Kraft 4} & \multirow[b]{2}{*}{ Pfahl } \\
\hline & & & Pfahl & horizontal & vertikal & \\
\hline & 86,2 & & 23,4 & 33,4 & 29,7 & 15,3 \\
\hline & 128,7 & 85,1 & $81,0^{*}$ & 16,2 & 64,1 & 61,2 \\
\hline \multirow[t]{2}{*}{ Verpflanzung } & 61,2 & 59,5 & 35,2 & & & \\
\hline & \multicolumn{6}{|c|}{ Anzahl (N) } \\
\hline Pflanzung & 20,9 & 5,7 & 1,1 & 16,3 & 6,1 & 0,9 \\
\hline Saat & 16,4 & 3,9 & 1,7 & 13,1 & 3,3 & 1,9 \\
\hline Verpflanzung & 18,8 & 7,5 & 2,0 & & & \\
\hline
\end{tabular}


Die Wurzeln der gepflanzten Eichen der Kraft'schen Klasse 2 haben horizontal eine Querschnittflächensumme von $86,2 \mathrm{~cm}^{2}$ und vertikal eine von $56,7 \mathrm{~cm}^{2}$. Das Verhältnis der Horizontal- zu den Vertikalwurzeln liegt demnach bei 3:2. Die Pfahlwurzel der gepflanzten Eichen macht $40 \%$ der gesamten vertikalen Wurzelquerschnittfläche aus. Die horizontalen und vertikalen Wurzeln der gepflanzten Eiche haben im Schnitt einen $40 \%$ kleineren Querschnitt als die vergleichbaren Eichen der Saat. Die horizontalen Wurzeln der Saateichen besitzen eine Querschnittfläche von $129 \mathrm{~cm}^{2}$, die vertikalen eine von $85 \mathrm{~cm}^{2}$. Das Verhältnis der Horizontalzu den Vertikalwurzeln liegt somit ebenfalls bei 3:2. Dabei hat die Pfahlwurzel an der Gesamtvertikalausbildung einen Anteil von $95 \%$.

Die Anzahl der Wurzeln beträgt bei den herrschenden gepflanzten Eichen in horizontaler 21 und in vertikaler Richtung sechs. Die Saateichen besitzen horizontal 16 und vertikal vier Wurzeln. Bei ihnen wird mit der geringeren Anzahl von Vertikalwurzeln eine große Querschnittfläche erreicht.

Die horizontalen Wurzeln der herrschenden Bäume des verpflanzten Bestandes sind $3 \%$ dicker als die Vertikalwurzeln. An der Gesamtbewurzelung haben die $60 \mathrm{~cm}^{2}$ dicken Horizontalwurzeln einen Querschnittsflächenanteil von $51 \%$, die $61 \mathrm{~cm}^{2}$ starken Vertikalwurzeln einen Anteil von $49 \%$. Die Pfahlwurzel macht $57 \%$ der Vertikalbewurzelung aus. In der Horizontalen besitzen die herrschenden Eichen des verpflanzten Bestandes 19 Wurzeln. Vertikal sind es rund $50 \%$ weniger.

Bei den beherrschten gepflanzten Eichen differieren die Querschnittswerte der horizontalen und der vertikalen Wurzeln nicht sehr stark. Der Anteil der Pfahlwurzel an der gesamten vertikalen Wurzelquerschnittfläche liegt bei $50 \%$. Die Horizontalwurzeln haben einen prozentualen Anteil an der Gesamtbewurzelung von $73 \%$, die vertikalen einen von $27 \%$.

Die beherrschten Bäume des Saatbestandes weisen im Vergleich zu den Wurzeln der herrschenden Eichen eine stärkere Vertikal- als Horizontalbewurzelung auf. Die horizontale Wurzelquerschnittflächensumme pro Baum ist $75 \%$ geringer als die vertikale Fläche. Die Pfahlwurzel hat auch hier einen $96 \%$ igen Anteil an der Vertikalbewurzelung. Die hohe Querschnittfläche der Vertikalwurzeln setzt sich aus rund drei Wurzeln zusammen, die der horizontalen Wurzeln aus 13 Wurzeln.

Zusammenfassend kann gesagt werden, daß durchschnittlich die Saateiche im Vergleich zur gepflanzten Eiche rund $30 \%$ weniger Wurzeln hat, aber eine $40 \%$ größere Wurzelquerschnittfläche (Abb. 22). Dies liegt im wesentlichen an den Bäumen der Kraft'schen Klasse 4. Die beherrschten Saateichen besitzen in der Tiefe rund $50 \%$ weniger Wurzeln als die gepflanzten Eichen bei einer mehr als doppelt so großen Querschnittfläche.

Die Pfahlwurzelquerschnittfläche der herrschenden verpflanzten Eichen ist geringer als die der herrschenden Saateichen. Gegenüber den herrschenden Bäumen der gepflanzten Bäume weisen letztere knapp knapp die vierfachen Werte auf. Bei den beherrschten Bäumen macht die Pfahlwurzel der gesäten Eichen nahezu die gesamte vertikale Bewurzelung aus und bei den gepflanzten nur $50 \%$. 


\section{Kattenbühl}

Absolut liegen die Wurzelquerschnittwerte horizontal zwischen $20 \mathrm{~cm}^{2}$ und $50 \mathrm{~cm}^{2}$ und vertikal bei $8 \mathrm{~cm}^{2}$ (Abb. 23). Das Wurzelsystem der gepflanzten Eiche weist horizontal eine $16 \%$ größere Querschnittfläche auf als das der Saat I- und eine signifikant $(55 \%)$ größere als das der Saat II-Fläche. In der Vertikalen zeigt die Eiche der Saat I-Fläche den höchsten Querschnittflächenwert der Wurzeln. Er ist $13 \%$ bzw. $28 \%$ größer als die vergleichbaren Werte der Pflanzund Saat II-Eichen. Der Variationskoeffizient schwankt bei den horizontalen Wurzelquerschnittflächen zwischen $68 \%$ und $84 \%$. Dagegen erreicht er für die vertikalen Wurzeln aller Bestände Werte von $95 \%$.

Die Anzahl der Horizontalwurzeln ist bei den Eichen der Pflanzung um $19 \%$ größer als bei denen des Saat I-Bestandes (Abb. 23). Die Bäume der letztgenannte Gruppe zeigen darüber hinaus signifikant (39\%) mehr Horizontalwurzeln als die Saat II-Eichen. Vertikal weisen die gepflanzten Eichen doppelt soviele Wurzeln auf wie die der Saat I- und $50 \%$ mehr als die der Saat II-Eichen. Absolut sind in der Horizontalen zwischen 20 und 40 Wurzeln zu finden und in der Vertikalen zwischen drei und sechs . Die Streuung der Wurzelanzahl differiert horizontal zwischen $34 \%$ (Saat I) und $60 \%$ (Pflanzung) sowie vertikal zwischen $58 \%$ (Saat II) und $68 \%$ (Pflanzung).

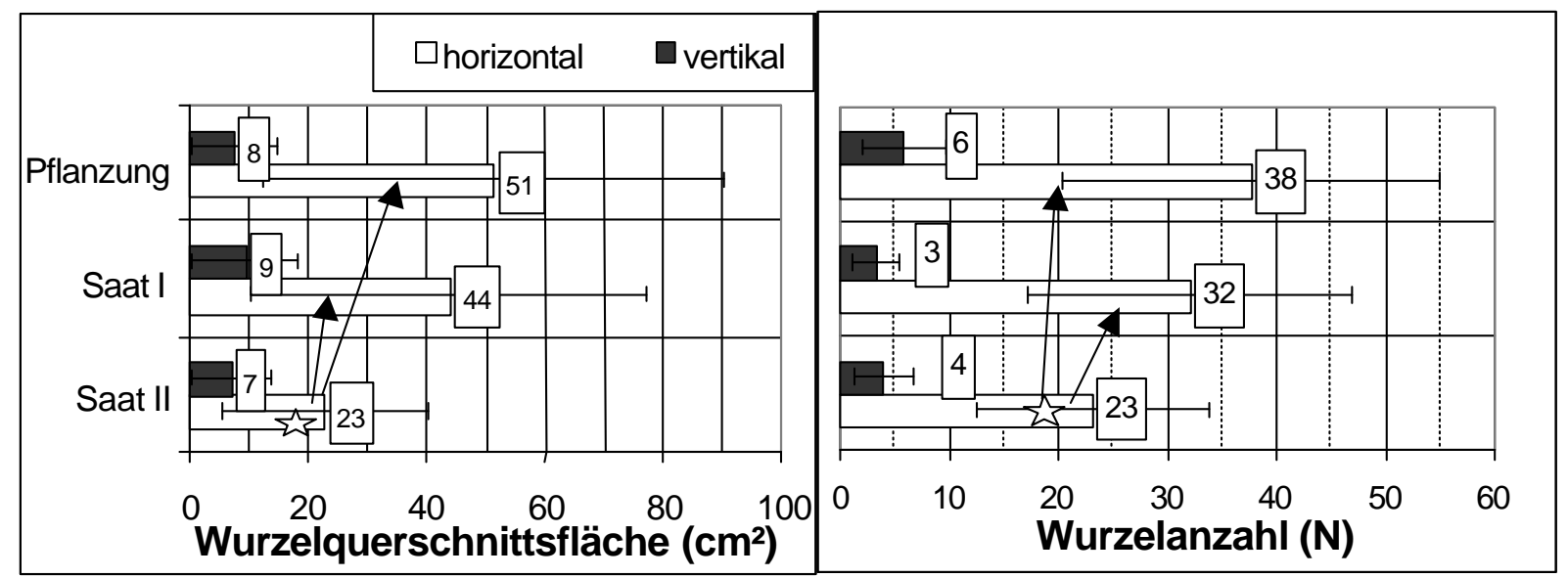

Abb. 23: Summe der Wurzelquerschnittfläche und Wurzelanzahl pro Baum und Standardabweichung (1. Sektion); Kattenbühl, 13 jährige Eiche (ఓ̌ssignifikant $\longrightarrow z u$ )

\subsubsection{Verteilung der Wurzelanzahl und Wurzelquerschnittfläche auf die drei horizontalen und vertikalen Sektionen am Beispiel der Eiche; Holzmin- den}

Die Verteilung der Wurzelanzahl und Wurzelquerschnittfläche wird nachfolgend beispielhaft an den 22 jährigen Eichen aus dem Untersuchungsgebiet in Holzminden erörtert. Anschließend werden tabellarisch für die anderen Flächen die prozentualen Anteile der horizontalen (GHQ) oder vertikalen (GVQ) Wurzeln an der Gesamtquerschnittsfläche (GQF) aufgeführt (GHQ+GVQ=GQF). Gleiche Berechnungen werden für die Wurzelanzahl dargestellt. Bezugsgröße bildet dafür die gesamte vertikale oder horizontale Wurzelmenge (GHA; GVA). Absolute Werte können anhand des vorherigen Abschnitts berechnet werden. 
Abb. 24 zeigt die Wurzelanzahl und die Summe der Wurzelquerschnittfläche (pro Baum) für die Eichen des herrschenden Saatbestandes. Die Darstellung erfolgt getrennt nach HorizontalVertikal- und Pfahlwurzeln. Der Wert der Pfahlwurzel ist ein Bestandteil der Vertikalwurzel. Erhöhte Daten der Pfahlwurzelanzahl in der zweiten Sektion kommen dadurch zustande, daß die Hauptwurzel sich in viele kleine Wurzeln zergliedert. Die Abkürzungen HQF, VQF und PQF stehen für die horizontalen (HQF), vertikalen (VQF) und Pfahlwurzel (PQF)- Querschnittsflächen der einzelnen Sektionen. Die Summe aus HQF Sektion 1, HQF Sektion 2 und HQF Sektion 3 ergibt die gesamte horizontale Querschnittfläche (GHQ). Gleiches gilt für die vertikale Wurzelrichtung (VQF). Nach demgleichen Schema wird die Anzahl der Wurzeln pro Wurzelsystem dargestellt.

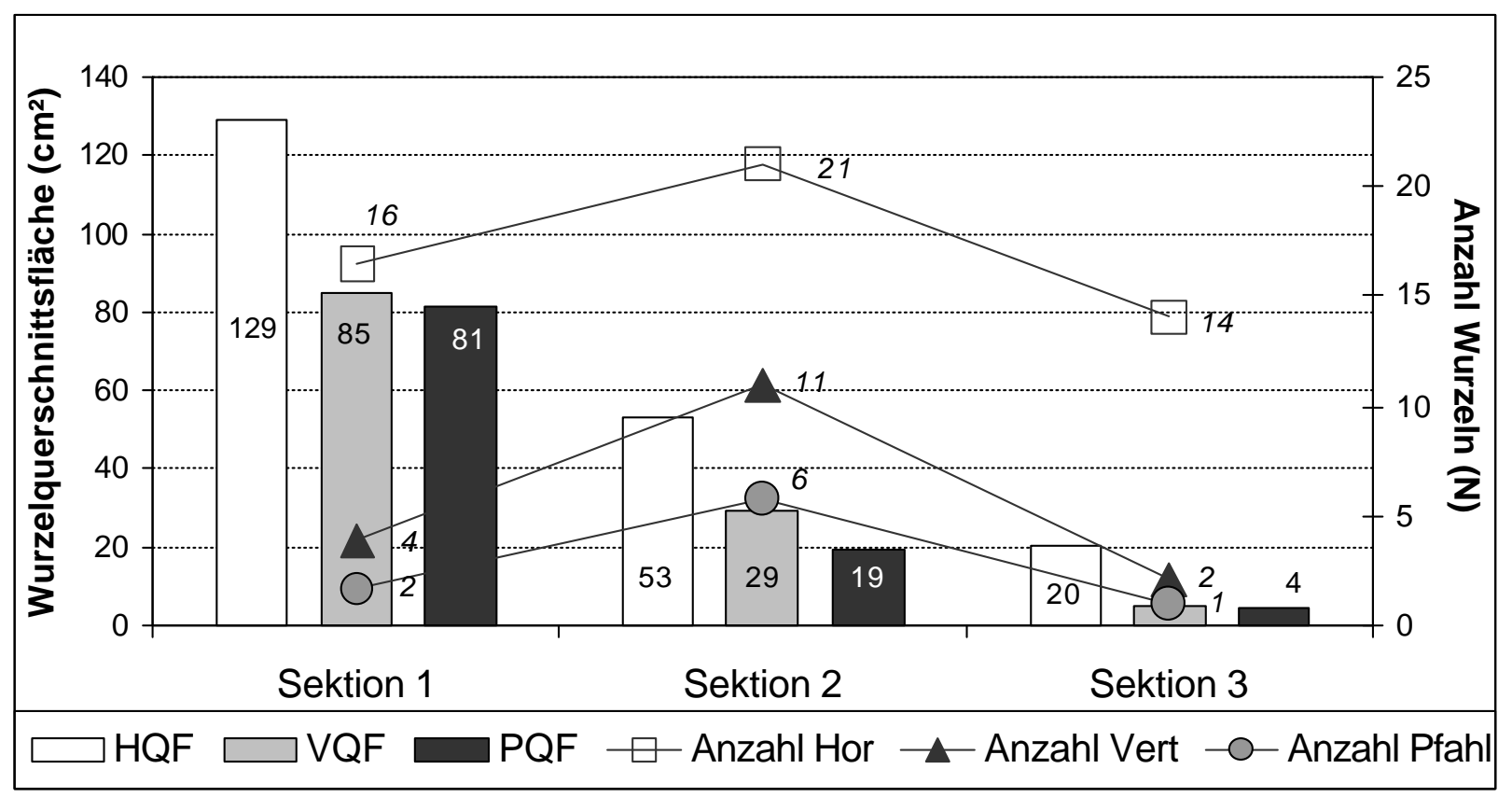

Abb. 24: Mittlere Wurzelquerschnittfläche und Wurzelanzahl der horizontalen (HQF), vertikalen (HQF) und Pfahlwurzeln (PQF) eines Wurzelsystems differenziert nach 3 Sektionen, Holzminden, Eiche, Kraft 2. Die große Anzahl der Pfahlwurzeln resultiert aus der Zergliederung der Pfahlwurzel in tieferen Bodenschichten

Bei den herrschenden Eichen aller in Holzminden untersuchten Bestände sind die Wurzeln folgendermaßen verteilt. Es liegen in der horizontalen stammnahen Region rund $60 \%$ (HQF Sektion 1), in der mittleren Sektion 30\% (HQF Sektion 2) und in der dritten Sektion $10 \%$ (HQF Sektion 3) der gesamten horizontalen Wurzelquerschnittfläche (GHQ) (Tabelle 36). In der Vertikalen entfällen etwa $70 \%$ (HVF Sektion 1) der gesamten Wurzelquerschnittfläche auf die erste, $25 \%$ (HVF Sektion 2) auf die zweite und $5 \%$ (HVF Sektion 3) auf die dritte Sektion. 
Tabelle 36: Mittlere prozentuale Verteilung der Wurzelquerschnittflächen auf die horizontalen und vertikalen Sektionen differenziert nach Kraft'schen Klassen; Holzminden, 22 jährige Eiche (HQF1+HQF2+HQF3=100\%; VQF1+VQF2+VQF3=100\%)

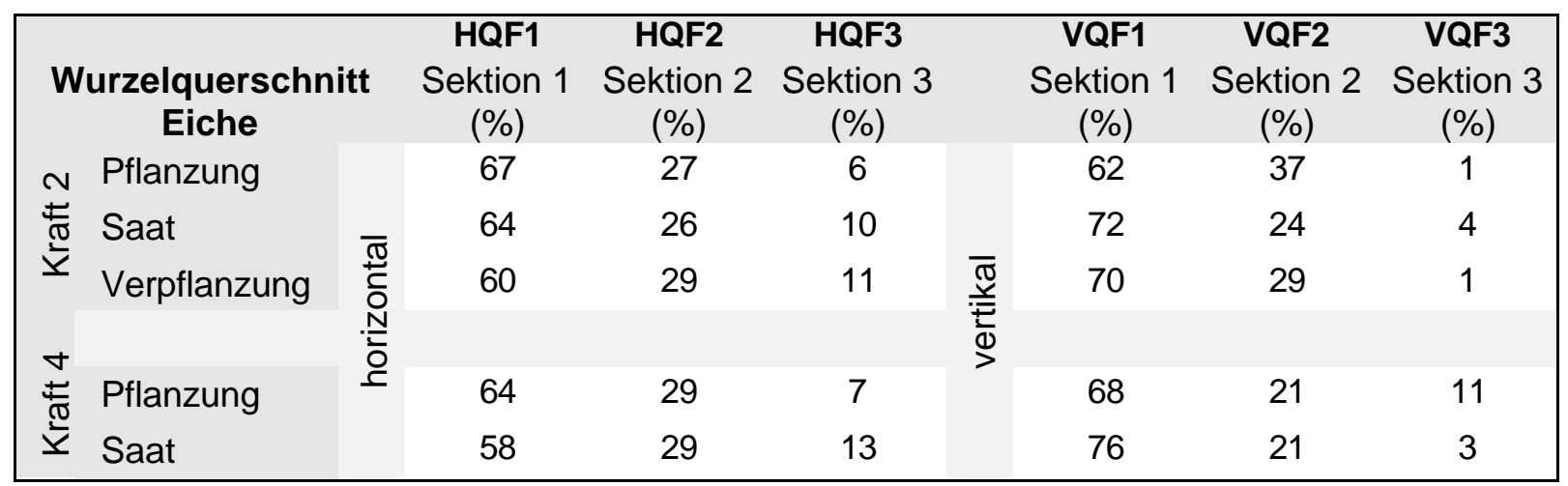

In Tabelle 37 werden für die Eichen der verschiedenen Bestände und Kraft'schen Klassen die prozentualen Anteile der Wurzeln pro Sektion an der Gesamtwurzelzahl dargestellt. Bezugsgröße ist die Summe der Wurzeln der drei Sektionen (GHA oder GVA).

Alle Eichen der verschiedenen Bestände weisen vertikal in der zweiten Sektion die meisten Wurzeln auf. In der Horizontalen sind die meisten Wurzeln in der ersten Sektion zu beobachten. In der Entfernung von $70 \mathrm{~cm}$ (HAF Sektion 3) sind relativ mehr Wurzeln zu finden als in einer Tiefe von $70 \mathrm{~cm}$ (VAF Sektion 3).

Tabelle 37: Mittlere prozentuale Verteilung der Wurzelanzahl auf die horizontalen und vertikalen Sektionen differenziert nach Kraft'schen Klassen; Holzminden, 22 jährige Eiche (HAF1+HAF2+HAF3=100\%; VAF1+VAF2+VAF3=100\%)

\begin{tabular}{|c|c|c|c|c|c|c|c|c|c|}
\hline \multirow{4}{*}{ 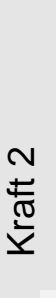 } & Wurzelanzahl & & $\begin{array}{c}\text { HAF1 } \\
\text { Sektion } 1 \\
(\%)\end{array}$ & $\begin{array}{c}\text { HAF2 } \\
\text { Sektion } 2 \\
(\%)\end{array}$ & $\begin{array}{c}\text { HAF3 } \\
\text { Sektion } 3 \\
(\%)\end{array}$ & & $\begin{array}{c}\text { VAF1 } \\
\text { Sektion } 1 \\
(\%)\end{array}$ & $\begin{array}{c}\text { VAF2 } \\
\text { Sektion } 2 \\
(\%)\end{array}$ & $\begin{array}{c}\text { VAF3 } \\
\text { Sektion } 3 \\
(\%)\end{array}$ \\
\hline & Pflanzung & \multirow{5}{*}{ 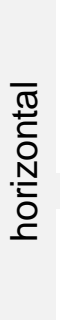 } & 42 & 37 & 21 & \multirow{5}{*}{$\frac{\overline{\mathscr{T}}}{\stackrel{\underline{T}}{\Phi}}$} & 30 & 51 & 18 \\
\hline & Saat & & 32 & 41 & 27 & & 23 & 65 & 13 \\
\hline & Verpflanzung & & 41 & 41 & 18 & & 43 & 49 & 6 \\
\hline \multirow{2}{*}{ 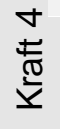 } & Pflanzung & & 45 & 40 & 15 & & 30 & 39 & 31 \\
\hline & Saat & & 59 & 27 & 14 & & 18 & 71 & 5 \\
\hline
\end{tabular}




\subsubsection{Tabellarische Darstellung der Verteilung der Wurzelquerschnittflächen und der Wurzelanzahl (Fi, $\mathrm{Bu}, \mathrm{Ki}, \mathrm{Ei})$ auf die horizontalen und vertikalen Sektionen}

Bei den untersuchten Fichten, Buchen, Kiefern und Eichen sind in der ersten Sektion sowohl horizontal als auch vertikal die stärksten Wurzeln zu finden (Tabelle 38). Die meisten Bäume besitzen stammnah (HQF Sektion 1) über zwei Drittel der Gesamtwurzelquerschnittfläche (GQF). Ausnahmen bilden die um 50 jährigen Fichten in Holzerode, Krebeck und Knesebeck. Hier gewinnt die zweite Sektion, gerade vertikal, an Bedeutung. Die Fichte des Reinbestandes aus Holzerode besitzt in der zweiten vertikalen Sektion an der gesamten vertikalen Wurzelquerschnittfläche (GVQ) einen Anteil von über $50 \%$.

Tabelle 38: Mittlere prozentuale Verteilung der Wurzelquerschnittfläche (WQF) auf die horizontalen und vertikalen Sektionen; Holzerode, Krebeck, Knesebeck und Kattenbühl; Fichte, Buche, Kiefer und Eiche

\begin{tabular}{|c|c|c|c|c|c|c|c|c|c|}
\hline & & & HQF1 & HQF2 & HQF3 & & VQF1 & VQF2 & VQF3 \\
\hline & WQF & & $\begin{array}{l}\text { 1. Sektion } \\
(\%)\end{array}$ & $\begin{array}{l}\text { 2. Sektion } \\
(\%)\end{array}$ & $\begin{array}{l}\text { 3. Sektion } \\
(\%)\end{array}$ & & $\begin{array}{c}\text { 1. Sektion } \\
(\%)\end{array}$ & $\begin{array}{l}\text { 2. Sektion } \\
(\%)\end{array}$ & $\begin{array}{l}\text { 3. Sektion } \\
(\%)\end{array}$ \\
\hline 0 & Buche & & 67 & 30 & 3 & & 65 & 31 & 4 \\
\hline 8 & $\mathrm{Bu} / \mathrm{Fi}$ & & 67 & 28 & 5 & & 68 & 28 & 4 \\
\hline$\frac{N}{0}$ & Fichte & & 53 & 35 & 12 & & 36 & 55 & 9 \\
\hline & $\mathrm{Fi} / \mathrm{Bu}$ & & 55 & 36 & 9 & & 56 & 33 & 10 \\
\hline 등 & Fichte 20 & & 75 & 20 & 5 & & 67 & 25 & 8 \\
\hline$\frac{0}{0}$ & Fichte 30 & & 73 & 23 & 4 & & 69 & 27 & 4 \\
\hline$\grave{\Sigma}$ & Fichte 41 & & 68 & 27 & 5 & & 56 & 37 & 7 \\
\hline & Fichte 20 & $\stackrel{\frac{\pi}{c}}{\frac{0}{n}}$ & 78 & 18 & 4 & $\overline{\widetilde{T}}$ & 94 & 3 & 3 \\
\hline ov & Fichte 31 & $\frac{\pi}{\frac{\pi}{0}}$ & 67 & 25 & 8 & 迺 & 65 & 28 & 7 \\
\hline Oి & Fichte 50 & & 62 & 29 & 9 & & 58 & 34 & 8 \\
\hline 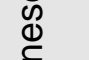 & Kiefer 20 & & 76 & 19 & 5 & & 82 & 16 & 2 \\
\hline 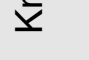 & Kiefer 33 & & 77 & 20 & 3 & & 72 & 24 & 4 \\
\hline & Kiefer 47 & & 74 & 22 & 4 & & 61 & 29 & 10 \\
\hline & Pflanzung & & 63 & 24 & 13 & & 95 & 5 & 0 \\
\hline$\frac{0}{\frac{1}{0}} \frac{\mathbb{d}}{0} \mathrm{c}$ & Saat I & & 64 & 24 & 12 & & 89 & 11 & 0 \\
\hline$\stackrel{\widetilde{T}}{\square}$ & Saat II & & 67 & 25 & 8 & & 83 & 17 & 0 \\
\hline
\end{tabular}

Die Anzahl der Wurzeln verteilt sich auf die drei Sektionen im Gegensatz zu den Wurzelquerschnittflächen sehr unterschiedlich (Tabelle 39). In der Regel sind in der zweiten Sektion die meisten Wurzeln zu finden. Ein Ausnahme bilden die 13 jährigen Eichenbestände aus Kattenbühl, bei denen stammnah (20 cm Radius; $30 \mathrm{~cm}$ Tiefe) die höchste Wurzelanzahl vorkommt.

In Holzerode sind bei den Buchen im Mischbestand rund $30 \%$ der Wurzeln in der ersten Sektion (HAF und VAF Sektion 1) und $50 \%$ in der zweiten zu finden. Bei den anderen Beständen befinden sich maximal $20 \%$ der Wurzeln in der ersten Sektion und $80 \%$ in der zweiten plus dritten Sektion. Dabei zeigen die Fichten in der dritten Sektion $10 \%$ bis $20 \%$ mehr Wurzeln als die Buchen. 
In Krebeck steigt der horizontale Querschnittflächenanteil der weiter von der Stammbasis entfernten Wurzeln mit Alterung an.

In Knesebeck geht in Abhängigkeit vom Alter der Anteil der ersten Sektion an der Gesamtwurzelanzahl zurück. Dies fällt besonders in der vertikalen Wurzelrichtung auf.

Tabelle 39: Mittlere prozentuale Verteilung der Wurzeln auf die horizontalen und vertikalen Sektionen; Holzerode, Krebeck, Knesebeck und Kattenbühl; Fichte, Buche, Kiefer und Eiche

\begin{tabular}{|c|c|c|c|c|c|c|c|c|c|}
\hline & $\mathrm{HAF}$ & HAF & HAF & & VAF & VAF & VAF \\
\hline \multirow{5}{*}{$\begin{array}{l}\frac{0}{0} \\
\frac{0}{D} \\
\text { N } \\
\text { 온 }\end{array}$} & \multicolumn{2}{|c|}{ Vurzelanzahl } & $\begin{array}{c}\text { 1. Sektion } \\
(\%)\end{array}$ & $\begin{array}{c}\text { 2. Sektion } \\
(\%)\end{array}$ & $\begin{array}{c}\text { 3. Sektion } \\
(\%)\end{array}$ & & $\begin{array}{c}\text { 1. Sektion } \\
(\%)\end{array}$ & $\begin{array}{l}\text { 2. Sektion } \\
(\%)\end{array}$ & $\begin{array}{c}\text { 3. Sektion } \\
(\%)\end{array}$ \\
\hline & Buche & \multirow{16}{*}{ 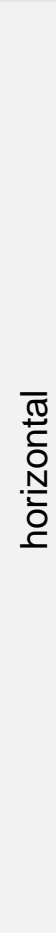 } & 18 & 47 & 35 & \multirow{16}{*}{ 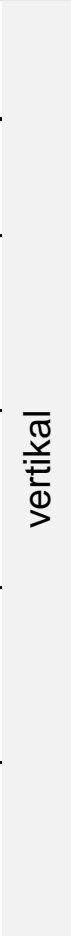 } & 12 & 59 & 29 \\
\hline & $\mathrm{Bu} / \mathrm{Fi}$ & & 32 & 46 & 22 & & 30 & 54 & 16 \\
\hline & Fichte & & 18 & 29 & 53 & & 14 & 49 & 37 \\
\hline & $\mathrm{Fi} / \mathrm{Bu}$ & & 15 & 38 & 47 & & 10 & 41 & 49 \\
\hline \multirow{3}{*}{ 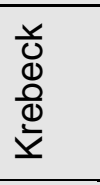 } & $\mathrm{Fi} 20$ & & 44 & 34 & 22 & & 48 & 40 & 12 \\
\hline & $\mathrm{Fi} 30$ & & 33 & 40 & 27 & & 25 & 57 & 18 \\
\hline & $\mathrm{Fi} 41$ & & 24 & 39 & 37 & & 15 & 55 & 30 \\
\hline \multirow{6}{*}{ 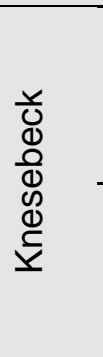 } & Fichte 20 & & 56 & 32 & 12 & & 75 & 16 & 9 \\
\hline & Fichte 31 & & 28 & 38 & 34 & & 34 & 45 & 21 \\
\hline & Fichte 50 & & 23 & 36 & 41 & & 16 & 52 & 32 \\
\hline & Kiefer 20 & & 62 & 27 & 11 & & 39 & 54 & 7 \\
\hline & Kiefer 33 & & 45 & 37 & 18 & & 42 & 41 & 17 \\
\hline & Kiefer 47 & & 34 & 41 & 25 & & 19 & 43 & 38 \\
\hline \multirow{3}{*}{ 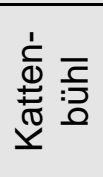 } & Pflanzung & & 60 & 30 & 10 & & 92 & 7 & 1 \\
\hline & Saat I & & 59 & 32 & 9 & & 83 & 16 & 1 \\
\hline & Saat II & & 60 & 31 & 9 & & 92 & 7 & 1 \\
\hline
\end{tabular}

\subsubsection{Verhältnis Horizontal- zu Vertikalwurzeln}

Im folgenden wird die Verteilung der Wurzeln in dem rund $3 \mathrm{~m}^{3}$ (bei den Eichen $1 \mathrm{~m}^{3}$ ) großen Wurzelballen beschrieben $\left(\left(\mathrm{Pi}^{*} \mathrm{r}^{2}\right)^{*}\right.$ Bodentiefe $)$. Sie wird anhand der Relationen zwischen Horizontal- und Vertikalbewurzelung in Anzahl und Querschnittfläche dargestellt. Ein enges Verhältnis zwischen der Summe der horizontalen und der der vertikalen Wurzelquerschnittfläche einer Sektion läßt auf ein gleichmäßiges Tiefenwurzelsystem schließen. Eine weite Relation bedeutet, daß eine geringere Wurzelquerschnittfläche bei den Vertikalwurzeln zu finden ist als bei den Horizontalwurzeln und somit ein Flachw urzelsystem ausgebildet wird.

Bei allen untersuchten Bäumen ist kein signifikanter Unterschied der Relationen mit Abfall der sozialen Stellung festzustellen.

\section{Holzerode}

Beim Vergleich aller Bestände wird von der ersten zur zweiten Sektion die Querschnittsflächenrelation enger und zur dritten Sektion hin wieder weiter (Tabelle 40). Eine Ausnahme bildet die Mischbestandsfichte, bei der die vertikale im Verhältnis zur horizontalen Querschnitt- 
fläche bei der zweiten Sektion abnimmt. In der dritten Sektion steigt sie jedoch wieder an. Demzufolge stellt sich die Verteilung in der zweiten Sektion weiter dar als in der dritten.

Deutliche Unterschiede sind zwischen Buchen und Fichten zu beobachten. Bei der Buche ist die Summe der Querschnittfläche gleichmäßiger verteilt als bei der Fichte. Dabei weist die Buche im Mischbestand weitere Relationen auf (2,6:1) als die im Reinbestand (1,4:1).

Bei den Fichtenbeständen besteht ein erheblicher Gegensatz in der Verteilung der Wurzelquerschnittflächen. Über alle Sektionen gemittelt, weist die Fichte des Reinbestandes ein Verhältnis der horizontalen zu vertikalen Querschnittsflächen von 13,9:1 auf. Die Fichte im Mischbestand zeigt hingegen Werte von 3,5:1. Erstere besitzt demnach fast 14 mal so große horizontale als vertikale Querschnittsflächen. Letztere 3,5mal so starke Horizontal- als Vertikalwurzeln. Dies ist ein deutliches Merkmal des Senkerwurzelsystems der Bäume des Mischbestandes.

Tabelle 40: Mittlere Relation der horizontalen zur vertikalen Wurzelquerschnittfläche (WQF); Holzerode, Fichte und Buche im Rein- und Mischbestand

\begin{tabular}{|lcccc|}
\hline WQF & Buche & Buche / Fichte & Fichte & Fichte / Buche \\
\hline Sektion 1 & $1,3: 1$ & $2,6: 1$ & $13,6: 1$ & $3,5: 1$ \\
Sektion 2 & $1,4: 1$ & $2,3: 1$ & $9,7: 1$ & $3,9: 1$ \\
Sektion 3 & $1,3: 1$ & $2,8: 1$ & $18,5: 1$ & $3,2: 1$ \\
\hline Mittel der Sektionen (sx) & $1,4: 1(0,08)$ & $2,6: 1(0,24)$ & $13,9: 1(4,37)$ & $3,5: 1(0,37)$ \\
\hline
\end{tabular}

Beim Betrachten der Wurzelanzahl der vier in Holzerode untersuchten Bestände kann für die zweite Sektion ein engeres Verhältnis der horizontalen zu den vertikalen Wurzeln gefunden werden als für die anderen Sektionen (Tabelle 41). Die Buchen des Reinbestandes und die Fichten des Mischbestandes zeigen genausoviele Vertikal- wie Horizontalwurzeln. Im Mittel der drei Sektionen stellt sich deren Relation mit Werten von 0,9:1 (Buche) und 1,0 : 1 (Fichte / Buche) enger dar als die Relationen der beiden anderen Flächen. Die Buche im Mischbestand weist bei durchschnittlichen Ergebnissen von 1,8:1 knapp doppelt soviele Horizontalals Vertikalwurzeln auf. Bei der Fichte im Reinbestand erhöht sich dieser Wert im Schnitt auf 2,5: 1. Diese Daten kommen durch die weite Relation in der ersten und dritten Sektion zustande.

Tabelle 41: Mittlere Relation der Horizontal- zu den Vertikalwurzeln (Anzahl); Holzerode, Fichte und Buche

\begin{tabular}{|llccc|}
\hline Anzahl & Buche & Buche / Fichte & Fichte & Fichte / Buche \\
\hline Sektion 1 & $1,24: 1$ & $2,16: 1$ & $2,95: 1$ & $1,62: 1$ \\
Sektion 2 & $0,72: 1$ & $1,49: 1$ & $1,42: 1$ & $0,79: 1$ \\
Sektion 3 & $0,89: 1$ & $1,75: 1$ & $3,17: 1$ & $0,72: 1$ \\
\hline Mittel der Sektionen (sx) & $0,95: 1(0,27)$ & $1,80: 1(0,33)$ & $2,51: 1(0,95)$ & $1,04: 1(0,50)$ \\
\hline
\end{tabular}

Zusammenfassend kann gesagt werden, daß die Buchen bei der Verteilung der Querschnittfläche und der Anzahl ein engeres Verhältnis Horizontal- zu Vertikalwurzeln haben als die Fichten. Die Fichte im Mischbestand nähert sich den Querschnittsflächenrelationen der Buche stark 
an, während die Fichte im Reinbestand eine wesentlich höhere Relation in Richtung Horizontalwurzeln aufweist als die Buche im Reinbestand. Dies trifft für die Wurzelanzahl nicht zu. Dies bedeutet, daß die Fichte im Reinbestand Vertikalwurzeln angelegt hat, diese aber sehr geringe Dimensionen erreichen.

Beim Vergleich der Buchenkollektive sind nicht so starke Unterschiede zwischen den Flächen festzustellen wie bei der Fichte. Die Buche im Mischbestand zeigt für die Querschnittfläche wie auch für die Anzahl geringfügig weitere Verteilungen der Wurzeln als die Buche im Reinbestand.

\section{Krebeck}

Das Wurzelquerschnittflächenverhältnis steigt in der ersten Sektion im Schnitt abhängig vom Alter von rund 6:1 auf 8:1 an (Tabelle 42). Die der zweiten Sektion liegt mit durchschnittlich $7: 1( \pm 0,4)$ bei allen drei Beständen auf einem ähnlichem Niveau. Die dritte Sektion zeigt in der Abfolge 21, 41 und 30 Jahre Verhältnisse zwischen den Horizontal- und Vertikalwurzeln von gerundet $5: 1$ bis $9: 1$.

Tabelle 42: Mittlere Relation der horizontalen zur vertikalen Wurzelquerschnittfläche (WQF); Krebeck, Fichte im Reinbestand

\begin{tabular}{|lllc|}
\hline WQF & Fichte 21 & Fichte 30 & Fichte 41 \\
\hline Sektion 1 & $5,9: 1$ & $5,9: 1$ & $7,7: 1$ \\
Sektion 2 & $7,1: 1$ & $6,7: 1$ & $7,4: 1$ \\
Sektion 3 & $5,0: 1$ & $9,5: 1$ & $8,6: 1$ \\
\hline Mittel der Sektionen (sx) & $6,0: 1(1,09)$ & $7,4: 1(1,93)$ & $7,9: 1(0,60)$ \\
\hline
\end{tabular}

Die mittlere Relation der Wurzelanzahl steigt in Abhängigkeit vom Alter bei der ersten Sektion von etwa 3,2:1 auf 4,0:1. Bei der zweiten und dritten Sektion ist ein gegenläufiger Trend zu beobachten (Tabelle 43). Das Verhältnis zwischen Horizontal- und Vertikalwurzeln nimmt bei der zweiten Sektion von den 21 jährigen zu den 41 jährigen Fichten von 2,2:1 auf 1,3:1 ab. Mit weiterer Entfernung vom Wurzelstock fällt das Verhältnis von 5,3:1 auf 2,4 :

Tabelle 43: Mittlere Relation der Horizontal- zu Vertikalwurzel (Anzahl); Krebeck, Fichte im Reinbestand

\begin{tabular}{|llll|}
\hline Anzahl & Fichte 21 & Fichte 30 & Fichte 41 \\
\hline Sektion 1 & $3,2: 1$ & $3,4: 1$ & $4,0: 1$ \\
Sektion 2 & $2,2: 1$ & $1,7: 1$ & $1,3: 1$ \\
Sektion 3 & $5,3: 1$ & $3,5: 1$ & $2,4: 1$ \\
\hline Mittel der Sektionen (sx) & $3,6: 1(1,60)$ & $2,8: 1(0,95)$ & $2,6: 1(1,34)$ \\
\hline
\end{tabular}

Die Wurzelquerschnitte der Horizontalen sind in $30 \mathrm{~cm}$ bis $40 \mathrm{~cm}$ Entfernung von der Stammbasis (erste Sektion) bei den Fichten sechs- bis siebenmal so stark, wie die der Vertikalen. Die jüngeren Bäume zeigen geringfügig ausgewogenere Verhältnisse als die älteren. Bei den Relationen der Wurzelanzahl weisen die älteren Fichten engere Werte auf. Demnach haben sie eine geringe Anzahl sehr starker Horizontalwurzeln. 


\section{Knesebeck}

Die Wurzelquerschnittflächen der beiden älteren Fichtenbestände weisen über alle Sektionen in horizontaler zu vertikaler Richtung Relationen von 5:1 bis 7:1 auf (Tabelle 44). Bei dem 20 jährigen Bestand steigen die entsprechenden Werte von der ersten zur zweiten Sektion auf maximal 42: 1 an. In der dritten Sektion fällt bei den jüngeren Fichten die Relation auf 20 mal stärkere Horizontal- als Vertikalwurzeln wieder ab.

Tabelle 45 stellt die Relation der horizontalen und vertikalen Wurzelanzahl für die Fichten dar. Bei der ersten Sektion sind horizontal ein- (31 jährige Fichte) bis zweimal (20- und 50 jährige Fichte) soviele Wurzeln zu finden als vertikal. Mit weiterer Entfernung vom Wurzelstock zeigen die jüngeren Bäume sieben- bis zehnmal soviele Horizontal- als Vertikalwurzeln. Ersterer Wert beschreibt die zweite Sektion in einer Distanz von $60 \mathrm{~cm}$ vom Stock, letzterer die dritte Sektion. In der zweiten Sektion fällt das Verhältnis bei den 31- und 50 jährigen Fichten auf rund 1,5: $1 \mathrm{ab}$. Die Anzahl der Vertikalwurzeln steigt somit an.

Die 20 jährige Kiefer besitzt in der ersten Sektion in der Horizontalen die gleiche Querschnittfläche wie in der Vertikalen (Tabelle 44). Dagegen sind entsprechende Werte der Horizontalwurzeln in einem Meter Entfernung vom Wurzelstock doppelt so groß wie die der Vertikalwurzeln (Tabelle 45). Die 33 jährige Kiefer hat in der ersten Sektion vertikal 2,6 mal so kleine Querschnittsflächen als horizontal. In der zweiten und dritten Sektion erhöhen sich die Werte der Vertikalwurzeln im Verhältnis zu denen der Horizontalwurzeln (1,8:1). Es sind dort 1,8 mal so große Horizontal- als Vertikalwurzelquerschnittflächen zu finden. Bei der 47 jährigen Kiefer ist dieser Effekt extremer zu beobachten. Hier nimmt das Verhältnis der Vertikal- zur Horizontalfläche von der ersten bis zur dritten Sektion zu. Das heißt, die Querschnittsflächen der Vertikalwurzeln sind in dieser weiteren Entfernung rund $25 \%$ größer als die der Horizontalwurzeln.

Tabelle 44: Mittlere Relation der horizontalen zur vertikalen Wurzelquerschnittfläche (WQF); Knesebeck, Fichte und Kiefer im Reinbestand

\begin{tabular}{|llll|llc|}
\hline WQF & Fichte 20 & Fichte 31 & Fichte 50 & Kiefer 20 & Kiefer 33 & Kiefer 47 \\
\hline Sektion 1 & $5,8: 1$ & $6,2: 1$ & $7,3: 1$ & $1,0: 1$ & $2,6: 1$ & $2,2: 1$ \\
Sektion 2 & $41,6: 1$ & $5,2: 1$ & $6,0: 1$ & $1,0: 1$ & $1,7: 1$ & $1,4: 1$ \\
Sektion 3 & $21,1: 1$ & $6,1: 1$ & $7,5: 1$ & $2,1: 1$ & $1,9: 1$ & $0,8: 1$ \\
\hline Mittel der & $22,9: 1(17,96)$ & $5,8: 1(0,54)$ & $6,9: 1(0,82)$ & $1,4: 1(0,63)$ & $2,1: 1(0,46)$ & $1,4: 1(0,72)$ \\
Sektionen (sx) & \multicolumn{3}{c}{ in Klammern Standardabweichung }
\end{tabular}

Für die Relationen der Vertikal- zu den Horizontalwurzeln in ihrer Anzahl ist für die Kiefer ein deutlicher Trend über alle drei Altersstufen festzustellen (Tabelle 45). Stammnah sind drei- bis viermal soviele Horizontal- wie Vertikalwurzeln vorhanden. In der zweiten Sektion ist ein Verhältnis von $1: 1$ bis 2:1 festzustellen. In der dritten Sektion wird der Altersunterschied deutlich. Die Kiefern des jüngsten Bestandes weisen vier mal soviele Horizontal- als Vertikalwurzeln auf, die des mittelalten zeigen nur noch zweimal soviele und die des ältesten Bestandes rund ein Fünftel weniger Horizontal- als Vertikalwurzeln. 
Tabelle 45: Mittlere Relation der Horizontal- zu den Vertikalwurzeln (Anzahl); Knesebeck, Fichte und Kiefer

\begin{tabular}{|c|c|c|c|c|c|c|}
\hline Anzahl & Fichte 20 & Fichte 31 & Fichte 50 & Kiefer 20 & Kiefer 33 & Kiefer 47 \\
\hline Sektion 1 & $3,4: 1$ & $2,1: 1$ & $3,2: 1$ & $3,9: 1$ & $3,3: 1$ & $3,5: 1$ \\
\hline Sektion 2 & $6,5: 1$ & $1,5: 1$ & $1,4: 1$ & $1,3: 1$ & $1,7: 1$ & $1,2: 1$ \\
\hline Sektion 3 & $9,5: 1$ & $2,3: 1$ & $2,3: 1$ & $3,6: 1$ & $2,0: 1$ & $0,8: 1$ \\
\hline $\begin{array}{l}\text { Mittel der } \\
\text { Sektionen (sx) }\end{array}$ & $6,5: 1 \quad(3,04)$ & $2,0: 1 \quad(0,41)$ & $2,3: 1 \quad(0,88)$ & $2,9: 1 \quad(1,44)$ & $2,3: 1 \quad(0,87)$ & $1,8: 1 \quad(1,48)$ \\
\hline
\end{tabular}

in Klammern Standardabweichung

Die Relationen zwischen den Horizontal- und Vertikalwurzeln kennzeichnen sehr deutlich das für die Kiefer charakteristische Pfahlwurzelsystem.

\section{Holzminden}

Die Relationen der horizontalen zu den vertikalen Wurzelquerschnittflächen werden für die Eichen differenziert nach sozialen Stellungen dargestellt und erörtert.

In der ersten und zweiten Sektion ist die Wurzelquerschnittfläche bei fast allen Beständen in der Horizontalen bis zu einmal größer als in der Vertikalen (Tabelle 46). Bei der dritten Sektion des verpflanzten Bestandes steigt die Relation auf 10:1. Die beherrschten Saateichen weisen in der ersten und in der zweiten Sektion höhere vertikale als horizontale Wurzelquerschnittflächen auf. Bei den Wurzeln der beherrschten Eichen des gepflanzten Bestandes ist dies nicht der Fall. Dort sind die Werte der horizontalen Wurzeln genauso bis doppelt so groß wie die vertikalen.

Tabelle 46: Mittlere Relation der horizontalen zur vertikalen Wurzelquerschnittfläche (WQF) differenziert nach Kraft'schen Klassen; Holzminden, 22 jährige Eiche

\begin{tabular}{|c|c|c|c|c|c|c|}
\hline WQF & Pflanzung & $\begin{array}{l}\text { Saat } \\
\text { Kraft } 2\end{array}$ & Verpflanzung & $\begin{array}{r}\text { Pflanzung } \\
\text { Kra }\end{array}$ & $\begin{array}{l}\text { Saat } \\
\text { ft } 4\end{array}$ & \\
\hline Sektion 1 & $1,5: 1$ & $1,5: 1$ & $1,0: 1$ & $1,1: 1$ & $0,3: 1$ & \\
\hline Sektion 2 & $1,6: 1$ & 1,9:1 & $1,2: 1$ & $1,7: 1$ & $0,3: 1$ & \\
\hline Sektion 3 & $2,0: 1$ & $4,1: 1$ & $10,4: 1$ & $5,6: 1$ & $3,2: 1$ & \\
\hline $\begin{array}{l}\text { Mittel der } \\
\text { Sektionen (sx) }\end{array}$ & $1,7: 1 \quad(0,28)$ & $2,5: 1 \quad(1,41)$ & $4,2: 1 \quad(5,38)$ & $2,8: 1 \quad(2,45)$ & $1,3: 1$ & $(1,67)$ \\
\hline
\end{tabular}

Im Mittel der Sektionen besitzen die Wurzelsysteme der Eichen drei bis viermal soviele Horizontal- als Vertikalwurzeln (Tabelle 47). Dabei zeigen die verpflanzten Bäume eine hohe Streuung der Werte. Diese Streuung kommt durch die Relationen der dritten Sektion zustande, die 10,4 : 1 beträgt.

Bei allen Eichen sind in der ersten Sektion erheblich mehr Horizontal- als Vertikalwurzeln zu finden. Die entsprechenden Relationen werden zur zweiten Sektion hin enger und steigen zur dritten Sektion wieder an. Am deutlichsten ist dies bei den beherrschten Saateichen zu beobachten. Dort sind vertikal in der zweiten Sektion $25 \%$ mehr Wurzeln zu finden als horizontal. 
Tabelle 47: Mittlere Relation der Horizontal- zu den Vertikalwurzel (Anzahl); Holzminden, 22 jährige Eiche

\begin{tabular}{|lccc||cc|}
\hline Anzahl & Pflanzung & \multicolumn{1}{c||}{ Saat } & Verpflanzung & Pflanzung & Saat \\
& \multicolumn{3}{c}{ Kraft 2 } & & \multicolumn{2}{c|}{ Kraft 4 } \\
\hline Sektion 1 & $3,7: 1$ & $4,3: 1$ & $2,5: 1$ & $2,7: 1$ & $4,0: 1$ \\
Sektion 2 & $2,7: 1$ & $1,9: 1$ & $2,1: 1$ & $2,4: 1$ & $0,8: 1$ \\
Sektion 3 & $3,8: 1$ & $6,5: 1$ & $8,0: 1$ & $5,6: 1$ & $2,6: 1$ \\
\hline Mittel der & $3,4: 1(0,60)$ & $4,2: 1(2,31)$ & $4,2: 1(3,30)$ & $3,5: 1(1,76)$ & $2,5: 1(1,60)$ \\
Sektionen (sx) & & & & & \\
\hline
\end{tabular}

in Klammem Standardabweichung

Die Relationen zwischen Horizontal- und Vertikalwurzeln in Wurzelquerschnittfläche und Anzahl verdeutlichen das für die Eiche charakteristische Pfahlwurzelsystem. Besonders ausgeprägt ist dies bei den gesäten Bäumen der Kraft'schen Klasse 4 zu bemerken. Dort sind signifikante Unterschiede zwischen gesäten und gepflanzten Eichen festzustellen. Für die herrschenden Bäume kann dieses nicht nachgewiesen werden. Saat und Pflanzung unterscheiden sich nicht signifikant.

\section{Kattenbühl}

Tabelle 48 stellt die Relationen der horizontalen zu der vertikalen Wurzelquerschnittfläche und Anzahl dar. Bei diesen Beständen sind nur an einzelnen Bäumen Wurzeln in der dritten Sektion gefunden worden, daher findet für diese Distanz keine Verhältnisbetrachtung statt. Die gepflanzte Eiche besitzt für die erste und zweite Sektion etwa sechsmal soviele Horizontal- wie Vertikalwurzeln. Dabei zeigen die Horizontalwurzeln eine ebenfalls sechsmal so große Querschnittfläche. Die Saat I Eiche weist bei einer fünfmal so großen Horizontal- als Vertikalwurzelquerschnitt eine rund zehnmal so hohe Horizontalwurzelanzahl auf. Allerdings ist Erstgenanntes nur für die erste Sektion zutreffend. In der zweiten Sektion liegt das Querschnittsflächenverhältnis bei $10: 1$. Bei den Saat II Eichen sind in der ersten Sektion fünf mal mehr Wurzeln in der Horizontalen als in der Vertikalen zu finden. Ihr Wurzelquerschnittflächenverhältnis in der ersten Sektion zeigt den, von allen Beständen, geringsten Wert von 3 : 1 (Pfahlwurzel).

Tabelle 48: Mittlere Relation der Horizontal- zu den Vertikalwurzeln (Anzahl) sowie der horizontalen zur vertikalen Wurzelquerschnittfläche (WQF); Kattenbühl, 13 jährige Eiche

\begin{tabular}{|lccc|ccc|}
\hline \multicolumn{3}{c}{ WQF } & \multicolumn{3}{c|}{ Anzahl } \\
\hline & Pflanzung & Saat I & Saat II & Pflanzung & Saat I & Saat II \\
Sektion 1 & $6,7: 1$ & $4,7: 1$ & $3,29: 1$ & $6,4: 1$ & $9,6: 1$ & $5,7: 1$ \\
Sektion 2 & $6,0: 1$ & $9,1: 1$ & $7,5: 1$ & $6,3: 1$ & $9,9: 1$ & $6,9: 1$ \\
\hline
\end{tabular}




\subsubsection{Wurzelvolumen}

Durch die in Abschnitt 3.10.2.3 dargestellte Methode wurde das Wurzelvolumen berechnet.

\section{Holzerode}

Zur Bestimmung des Wurzelvolumens wurden für alle untersuchten Bäume Regressionsrechnungen durchgeführt (WQF in bezug zur Entfernung von der Stammbasis). Zwei Bäume in Holzerode werden aus der Betrachtung herausgenommen. Sie wiesen bei der Anpassung der Ausgleichsfunktionen ein Bestimmtheitsmaß unter 0,5 auf.

Die Horizontal-, Vertikal- und Gesamtwurzelvolumina werden in Tabelle 49 dargestellt. Signifikante Unterschiede des Wurzelvolumens sind zwischen Buche und Fichte festzustellen. Das größte Wurzelvolumen weist die Fichte im Mischbestand mit durchschnittlich $112 \mathrm{dm}^{3}$ pro Baum auf. Die entsprechenden Werte sind bei der Fichte im Reinbestand um $20 \%$, bei der Buche im Reinbestand um $54 \%$ und bei der im Mischbestand um $64 \%$ geringer. Ein Unterschied zwischen den Fichtenkollektiven läßt sich beim Betrachten der Vertikalwurzeln nachweisen. Dabei zeigt die Fichte im Mischbestand ein dreimal so großes Volumen als im Reinbestand. Sie besitzt vertikal ein ähnliches Wurzelvolumen wie die Buche im Reinbestand. Diese zeigt jedoch in der horizontalen Wurzelrichtung ein $68 \%$ geringeres Wurzelvolumen als die Fichte. Die Mischbestandsbuche weist horizontal nahezu das gleiche, vertikal dagegen $80 \%$ weniger Volumen auf wie die Reinbestandsbuche.

Mit Variationskoeffizienten bis zu $100 \%$ variieren die Volumenwerte der Vertikalwurzeln der Fichte im Mischbestand sehr.

Tabelle 49: Mittleres Horizontal-, Vertikal- und Gesamtwurzelvolumen je Baum mit Standardabweichung (sx); Holzerode, Fichte und Buche im Rein- und Mischbestand

\begin{tabular}{|lcccccccc|}
\hline Wurzelvolumen $\left(\mathbf{d m}^{\mathbf{3}}\right)$ & Buche & $\mathbf{( s x}$ & $\mathbf{B u} / \mathbf{F i}$ & $\mathbf{( s x )}$ & Fichte & $\mathbf{( s x )}$ & $\mathbf{F i} / \mathbf{B u}$ & $\mathbf{( s x})$ \\
\hline horizontal & 28,9 & $(15,4)$ & 28,1 & $(9,1)$ & 83,1 & $(29,8)$ & 90,5 & $(40,3)$ \\
vertikal & 22,4 & $(9,0)$ & 12,4 & $(11,9)$ & 6,8 & $(6,0)$ & 21,7 & $(21,9)$ \\
\hline Gesamt (hor + vert) & 51,3 & $(18,4)$ & 40,5 & $(17,1)$ & 89,9 & $(32,1)$ & 112,2 & $(47,2)$ \\
\hline
\end{tabular}

\section{Krebeck}

Die Wurzeln der jüngsten Fichten besitzen ein Gesamtvolumen von $9 \mathrm{dm}^{3}$ und die der ältesten eines von $50 \mathrm{dm}^{3}$ (Tabelle 50). Das Wurzelvolumen steigt in Abhängigkeit vom Alter der Fichten von $21 \mathrm{zu} 30$ Jahren auf nahezu das Dreifache und von $30 \mathrm{zu} 41$ Jahren auf mehr als das doppelte. Der Variationskoeffizient fällt vom jüngsten zum ältesten Bestand von $81 \%$ auf $65 \%$ ab.

Durchschnittlich entfallen $87 \%( \pm 2)$ des Gesamtvolumens auf die Horizontalwurzeln und $13 \%( \pm 2)$ auf die Vertikalwurzeln. Das Volumen der Horizontalwurzeln steigt von $21 \mathrm{zu}$ 30 Jahren auf etwa das Dreifache und von 30 zu 41 Jahren auf das Doppelte. Die Variationskoeffizienten liegen zwischen $66 \%$ und $85 \%$. Wiederum zeigt die älteste Fläche die geringste und die jüngste die höchste Variation. Etwas anders stellt sich das Volumen der Vertikalwurzeln dar. In Abhängigkeit vom Alter des Bestandes steigt das Volumen erst auf fast das Dreifache und dann auf mehr als das Doppelte. Der Variationskoeffizient erhöht sich in der Vertikalen auf über $100 \%$. 
Tabelle 50: Mittleres Horizontal-, Vertikal- und Gesamtwurzelvolumen je Baum mit Standardabweichung (sx); Krebeck, Fichte im Reinbestand

\begin{tabular}{|llccccc|}
\hline Wurzelvolumen $\left(\mathbf{d m}^{\mathbf{3}}\right)$ & $\mathbf{F i} \mathbf{2 1}$ & $\mathbf{( s x})$ & Fi 30 & $\mathbf{( s x )}$ & Fi 41 & (sx) \\
\hline horizontal & 7,5 & $(6,4)$ & 18,6 & $(13,2)$ & 41,7 & $(28,0)$ \\
vertikal & 1,1 & $(1,3)$ & 2,9 & $(3,5)$ & 5,0 & $(6,3)$ \\
\hline Gesamt (hor + vert) & 8,6 & $(7,0)$ & 21,5 & $(14,2)$ & 46,7 & $(30,2)$ \\
\hline
\end{tabular}

\section{Knesebeck}

Das Wurzelvolumen nimmt im Schnitt von $20 \mathrm{zu} 31$ Jahren von $1,8 \mathrm{dm}^{3}$ pro Fichte um mehr als das Zehnfache und von $31 \mathrm{zu} 50$ Jahren auf rund das Dreifache zu (Tabelle 51).

Tabelle 51: Mittleres Horizontal-, Vertikal- und Gesamtwurzelvolumen je Baum mit Standardabweichung (sx); Knesebeck; Fichte im Reinbestand

\begin{tabular}{|lcccccc|}
\hline Wurzelvolumen $\left(\mathbf{d m}^{\mathbf{3}}\right)$ & \multicolumn{2}{c}{ Fichte $\mathbf{2 0}(\mathbf{s x})$} & \multicolumn{2}{c|}{ Fichte $\mathbf{3 1} \mathbf{( s \mathbf { x } )}$} & \multicolumn{2}{c|}{ Fichte 50 (sx) } \\
\hline horizontal & 1,6 & $(1,5)$ & 17,1 & $(16,2)$ & 58,3 & $(38,5)$ \\
vertikal & 0,2 & $(0,2)$ & 3,2 & $(3,2)$ & 12,8 & $(7,2)$ \\
\hline Gesamt (hor + vert) & 1,8 & $(1,5)$ & 20,3 & $(18,6)$ & 71,1 & $(41,8)$ \\
\hline
\end{tabular}

Das Volumen der Horizontalwurzeln der Kiefern (Tabelle 52) steigt in Abhängigkeit vom Alter von 20 auf 33 Jahre um mehr als das Zehnfache an, bei den Vertikalwurzeln auf das Dreifache. Von $33 \mathrm{zu} 47$ Jahren erhöht sich das Horizontalwurzelvolumen auf das Fünffache und das Vertikalwurzelvolumen um das Zehnfache. Die absoluten Werte des Gesamtvolumens liegen zwischen $10 \mathrm{dm}^{3}$, bei der jüngsten und $50 \mathrm{dm}^{3}$ bei der ältesten Kiefer. Die größte Streuung der Werte ist bei den 33 jährigen Kiefern zu beobachten. Der entsprechende Variationskoeffizient liegt bei $90 \%$.

Tabelle 52: Mittleres Horizontal-, Vertikal- und Gesamtwurzelvolumen mit Standardabweichung (sx); Knesebeck, Kiefer im Reinbestand

\begin{tabular}{|lcccccc|}
\hline Wurzelvolumen $\left(\mathbf{d m}^{\mathbf{3}}\right)$ & Kiefer $\mathbf{2 0}$ & $\mathbf{( s x})$ & \multicolumn{2}{c|}{ Kiefer 33 $\mathbf{( s x )}$} & \multicolumn{2}{c|}{ Kiefer 47 (sx) } \\
\hline horizontal & 0,5 & $(0,6)$ & 6,7 & $(7,8)$ & 31,1 & $(25,1)$ \\
vertikal & 0,6 & $(0,4)$ & 1,7 & $(2,2)$ & 19,2 & $(10,9)$ \\
\hline Gesamt (hor + vert) & 1,1 & $(0,9)$ & 8,4 & $(8,1)$ & 50,3 & $(32,5)$ \\
\hline
\end{tabular}

\section{Holzminden}

Die Volumina der Horizontal- und Vertikalwurzeln werden, differenziert nach Kraft'scher Klasse, in Tabelle 53 und 54 dargestellt.

Der herrschenden Saateichen zeigen ein größeres Wurzelvolumen als die herrschenden Pflanzeichen. Dieses ist in der horizontalen Wurzelrichtung bei den Eichen der Saat signifikant höher als bei denen der Pflanzung, in der vertikalen hingegen nicht. Erstere besitzen ein Wurzelvolumen von $13,8 \mathrm{dm}^{3}$, letztere eines von $10,7 \mathrm{dm}^{3}$. Die verpflanzten Bäume weisen horizontal das halbe und vertikal ein $17 \%$ geringeres Wurzelvolumen auf als die des Saatbestandes. Mit Abfall der sozialen Stellung verringert sich das Wurzelvolumen bei den Eichen der Pflanzung um insgesamt $60 \%$. Bei der Saat zeigen die beherrschten Eichen horizontal $87 \%$ und vertikal 
$40 \%$ weniger Wurzelvolumen als die herrschenden. Die Unterschiede zwischen Saat- und Pflanzeichen der Kraft'schen Klasse 4 liegen bei $12 \%$.

Tabelle 53: Mittleres Horizontal-, Vertikal- und Gesamtwurzelvolumen je Baum mit Standardabweichung (sx); Holzminden, Eiche, Kraft'sche Klasse 2 (*signifikant $\longrightarrow$ zu)

\begin{tabular}{|c|c|c|c|c|c|c|}
\hline Wurzelvolumen $\left(\mathrm{dm}^{3}\right)$ & $\begin{array}{l}\text { Pflanzung } \\
\text { Kraft } 2\end{array}$ & (sx) & $\begin{array}{l}\text { Saat } \\
\text { Kraft } 2\end{array}$ & (sx) & $\begin{array}{l}\text { Verpflanzung ( } \\
\text { Kraft } 2\end{array}$ & $(\mathbf{s x})$ \\
\hline horizontal & 8,1 & $(2,5) \star$ & $\rightarrow 12,7$ & $(3,4)$ & 6,2 & $(2,4)$ \\
\hline vertikal & 2,6 & $(1,6)$ & 3,6 & $(1,8)$ & 3,0 & $(2,1)$ \\
\hline Gesamt (hor + vert) & 10,7 & $(6,6)$ & 13,8 & $(4,6)$ & 9,2 & $(2,8)$ \\
\hline
\end{tabular}

Tabelle 54: Mittleres Horizontal-, Vertikal- und Gesamtwurzelvolumen je Baum mit Standardabweichung (sx); Holzminden, Eiche, Kraft'sche Klasse 4 ( ${ }^{*}$ signifikant $z u)$

\begin{tabular}{|llll|}
\hline Wurzelvolumen $\left(\mathbf{d m}^{3}\right)$ & $\begin{array}{c}\text { Pflanzung } \\
\text { Kraft 4 }\end{array}$ & \multicolumn{1}{c|}{$\begin{array}{c}\text { Saat } \\
\text { Kraft } 4\end{array}$} \\
\hline horizontal & $(\mathbf{s x})$ \\
vertikal & 3,3 & $(1,5) * 1,6$ & $(1,3)$ \\
\hline Gesamt (hor + vert) & 1,0 & $(0,5) \stackrel{* \rightarrow 2,2}{\longrightarrow}(0,7)$ \\
\hline
\end{tabular}

\section{Kattenbühl}

Mit 2,2 $\mathrm{dm}^{3}$ besitzen die gepflanzten Eichen das signifikant größte Wurzelvolumen (Tabelle 55). Im Schnitt zeigen sie in horizontaler Richtung ein um $27 \%$ größeres Wurzelvolumen als die gesäten Bäume (Saat I). Das mittlere Volumen der Vertikalwurzeln ist in allen Beständen etwa gleich groß. Bei den gepflanzten Eichen haben die Horizontalwurzeln einen durchschnittlichen Anteil von $86 \%$ am Geamtwurzelvolumen. Die entsprechenden Werte betragen bei den Eichen des Saat I Bestandes $83 \%$ und bei den Saat II Eichen $77 \%$. Die Vertikalwurzeln haben in derselben Reihenfolge Anteile von $14 \%, 17 \%$ und $23 \%$ am Gesamtwurzelvolumen.

Die Variationskoeffizienten des Gesamtwurzelvolumens liegen zwischen $71 \%$ und $76 \%$. Die Werte des vertikalen Wurzelvolumens sind größer als $100 \%$. Die des horizontalen Wurzelvolumens variieren bis zu $80 \%$.

Tabelle 55: Mittleres Horizontal-, Vertikal- und Gesamtwurzelvolumen je Baum mit Standardabweichung(sx); Kattenbühl, Eiche ( ${ }^{*}$ signifikant $\left.\longrightarrow z u\right)$

\begin{tabular}{|l|llllll|}
\hline Wurzelvolumen $\left(\mathbf{d m}^{3}\right)$ & Pflanzung & $\mathbf{( s x})$ & Saat I & $\mathbf{( s x )}$ & Saat II & $(\mathbf{s x})$ \\
\hline horizontal & 1,9 & $(1,5)^{*}$ & 1,5 & $(1,2)$ & 0,7 & $(0,5)$ \\
vertikal & 0,3 & $(0,5)$ & 0,3 & $(0,3)$ & 0,2 & $(0,3)$ \\
\hline Gesamt (hor + vert) & 2,2 & $(1,6){ }^{*}$ & 1,8 & $(1,3)$ & 0,9 & $(0,6)$ \\
\hline
\end{tabular}




\subsubsection{Wurzelvolumen differenziert nach sozialer Stellung}

In Abb. 25 wird beispielhaft für die Baumarten Fichte und Kiefer das Wurzelvolumen der Bäume unterschiedlicher sozialer Stellungen dargestellt. Bei den Fichten ist durchschnittlich von der ersten zur zweiten Kraft'schen Klasse eine Abnahme des Volumens um $30 \%( \pm 10)$ festzustellen. Dies trifft ebenfalls für die nicht dargestellten Eichen zu. Die Kiefern zeigen in diesem Bereich eine um $20 \%( \pm 6)$ stärkere Verminderung. Mit weiterem Abfall der sozialen Stellung von Klasse zu Klasse findet bei allen Baumarten eine Reduktion des Wurzelvolumens erst um rund $50 \%$ und dann um $75 \%$ statt. Die Bäume der Kraft'schen Klasse 4 haben bezüglich ihres Wurzelvolumens im Vergleich zu den stärkeren Bäumen nur eine geringe Bedeutung. Bei allen Beständen liegt die Variation innerhalb der Kraft'schen Klassen zwischen $30 \%$ und $50 \%$. Je unterdrückter der Baum, desto größer ist die Streuung der Daten. Bei nahezu allen Beständen unterscheiden sich die Bäume der Kraft'schen Klassen 1 und 2 und die der Klassen 3 und 4 nicht signifikant. Zwischen diesen beiden Gruppen sind jedoch fast immer signifikante Unterschiede festzustellen.

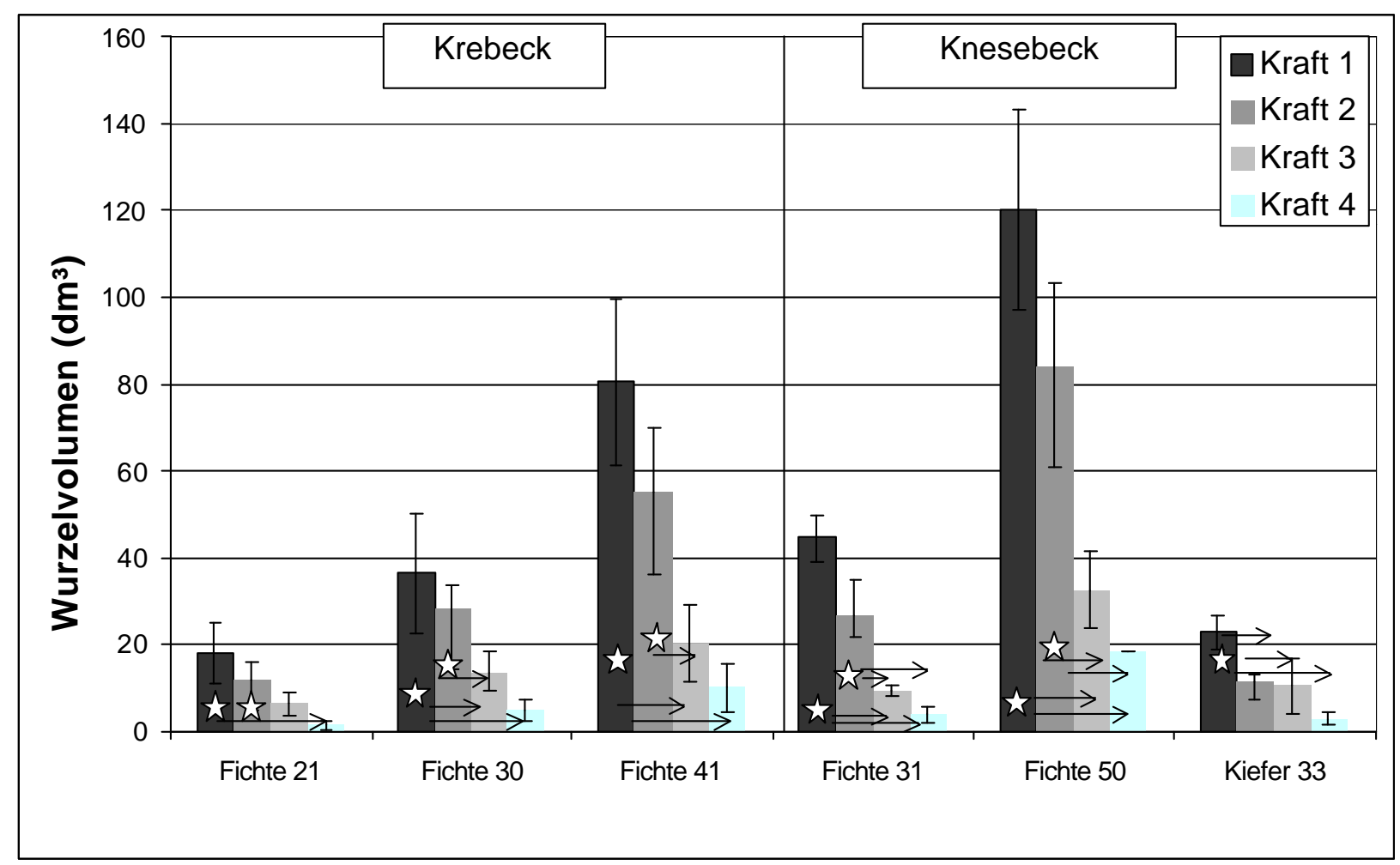

Abb. 25: Mittleres Wurzelvolumen mit Standardabweichung (sx) differenziert nach sozialer Stellung der Bäume ( ${ }^{3}$ signifikant $\longrightarrow$ zu); Krebeck und Knesebeck; Fichte und Kiefer 


\subsubsection{Wurzelvolumen der Probebäume in einem definierten Bodenraum}

Durch die Aufnahmetechnik wird für die Eiche ein rund $1 \mathrm{~m}^{3}$ großer Bodenraum beschrieben, der sich unterhalb des Wurzelstockes befindet. Die prozentualen Anteile, die die Wurzel in diesem Bodenraum einnimmt, sind beispielhaft für die Eiche aus Holzminden in Tabelle 56 dargestellt. Durchschnittlich ist ein sehr geringer Prozentsatz des Bodens mit Grobwurzeln durchzogen. Die Werte betragen bei den herrschenden Bäumen etwa $1 \%$ und bei den beherrschten $0,3 \%$ bis $0,4 \%$.

Tabelle 56: Mittlere Wurzelanteile (Vol. \%) pro $m^{3}$ Boden, differenziert nach Kraft'schen Klassen; Holzminden; 22 jährige Eiche

\begin{tabular}{|llll|}
\hline Kraft'sche Klasse 2 & Volumen (\%) & Kraft'sche Klasse 4 & Volumen (\%) \\
\hline Pflanzung & 0,97 & Pflanzung & 0,39 \\
Saat & 1,43 & Saat & 0,35 \\
Verpflanzung & 0,84 & & \\
\hline
\end{tabular}

Für die anderen Bestände sind die mittleren prozentualen Anteile der Grobwurzeln pro $\mathrm{m}^{3}$ Boden in Tabelle 57 dargestellt. Dabei durchwurzeln die älteren Fichten mit $0,6 \mathrm{~m}^{3}$ bis $1,4 \mathrm{~m}^{3} \mathrm{am}$ meisten Boden. Die etwa gleichalten $( \pm 10$ Jahre) Buchen zeigen eine halb so große Bodenerschließung.

Tabelle 57: Mittlere Wurzelanteile (Vol. \%) pro $m^{3}$ Boden

\begin{tabular}{|c|c|c|c|c|}
\hline \multicolumn{5}{|c|}{ Volumen (\%) pro $\mathrm{m}^{3}$ Boden } \\
\hline Holzerode & $\mathrm{Bu} / \mathrm{Bu}$ & $\mathrm{Bu} / \mathrm{Fi}$ & $\mathrm{Fi} / \mathrm{Fi}$ & $\mathrm{Fi} / \mathrm{Bu}$ \\
\hline Fichte und Buche & 0,6 & 0,5 & 1,1 & 1,4 \\
\hline \multirow{2}{*}{$\begin{array}{l}\text { Krebeck } \\
\text { Fichte }\end{array}$} & $\mathrm{Fi} 21$ & $\mathrm{Fi} 30$ & $\mathrm{Fi} 41$ & \\
\hline & 0,1 & 0,3 & 0,6 & \\
\hline \multirow{4}{*}{$\begin{array}{l}\text { Knesebeck } \\
\text { Fichte und Kiefer }\end{array}$} & Fichte 20 & Fichte 31 & Fichte 50 & \\
\hline & 0,02 & 0,3 & 0,9 & \\
\hline & Kiefer 20 & Kiefer 33 & Kiefer 47 & \\
\hline & 0,01 & 0,1 & 0,6 & \\
\hline \multirow{2}{*}{$\begin{array}{l}\text { Holzminden } \\
\text { Eiche }\end{array}$} & Pflanzung & Saat & Verpflanzung & \\
\hline & 0,7 & 0,8 & 0,8 & \\
\hline \multirow{2}{*}{$\begin{array}{l}\text { Kattenbühl } \\
\text { Eiche }\end{array}$} & Pflanzung & Saat I & Saat II & \\
\hline & 0,2 & 0,2 & 0,1 & \\
\hline
\end{tabular}

\subsubsection{Wurzeloberfläche}

Nach der Berechnung der für die Formel der Wurzeloberfläche notwendigen Parameter wird diese als Mittelwert kalkuliert. Durch die Multiplikation des Mittelwertes mit der Gesamtanzahl der Wurzeln pro Baum erhält man die Grobwurzeloberfläche eines Wurzelsystems. Durch 
die Mittelwertbetrachtung wird die Wurzeloberfläche tendenziell unterschätzt. Gerade die gering dimensionierten, oberflächenvergrößernden Wurzeln werden nicht betrachtet, so daß die berechneten Daten nur als grobe Schätzwerte aufgefaßt werden dürfen. Abb. 26 und Abb. 27 zeigen für Fichten-, Buchen- und Kiefernbestände die prozentualen und absoluten Anteile der Horizontal- und Vertikalwurzeln an der Gesamtoberfläche des Wurzelsystems. Abb. 28 und Abb. 29 stellen Entsprechendes für die Eichenbestände dar.

\section{Holzerode}

Die Fichten im Mischbestand haben eine Wurzeloberfläche von $749 \mathrm{dm}^{2}$. Dabei weisen sie eine $55 \%$ größere Wurzeloberfläche auf als die Fichten im Reinbestand. Diese besitzen eine $16 \%$ größere Wurzeloberfläche als die Buchen im Mischbestand und eine 6\% geringere als die im Reinbestand. Letztgenannte zeigen mit $40 \%$ den höchsten Anteil an vertikalen Wurzeln, die Fichte im Reinbestand zeigt mit $21 \%$ den geringsten.

\section{Krebeck}

Beim Vergleich der 21 und 30 jährigen Fichten erhöht sich die Wurzeloberfläche von $165 \mathrm{dm}^{2}$ um $67 \%$ auf $276 \mathrm{dm}^{2}$. Bis zum Alter von 41 Jahren vergrößert sie sich um $40 \%$ auf $387 \mathrm{dm}^{2}$. Der Anteil der Vertikalwurzeloberfläche an der Gesamtoberfläche fällt von 21- zu 41 jährig von etwa $20 \%$ auf $15 \%$ ab.

\section{Knesebeck}

Die Gesamtoberfläche der Wurzeln der Fichte steigt mit zunehmendem Alter von $20\left(64 \mathrm{dm}^{2}\right)$ auf 31 Jahre $\left(249 \mathrm{dm}^{2}\right)$ auf das Vierfache und von 31 auf $50 \mathrm{Jahre}\left(580 \mathrm{dm}^{2}\right)$ auf mehr als das Doppelte.

Die Wurzeloberfläche der Kiefer vergrößert sich von $20\left(37 \mathrm{dm}^{2}\right)$ zu 30 Jahren $\left(167 \mathrm{dm}^{2}\right)$ um das Vierfache und von $33 \mathrm{zu} 47$ Jahren $\left(426 \mathrm{dm}^{2}\right)$ auf mehr als das Doppelte.

Differenziert nach Wurzelrichtung, liegt der Anteil der vertikalen Wurzeloberfläche bei den drei Fichten- und Kiefernbeständen bei $20 \%$. 


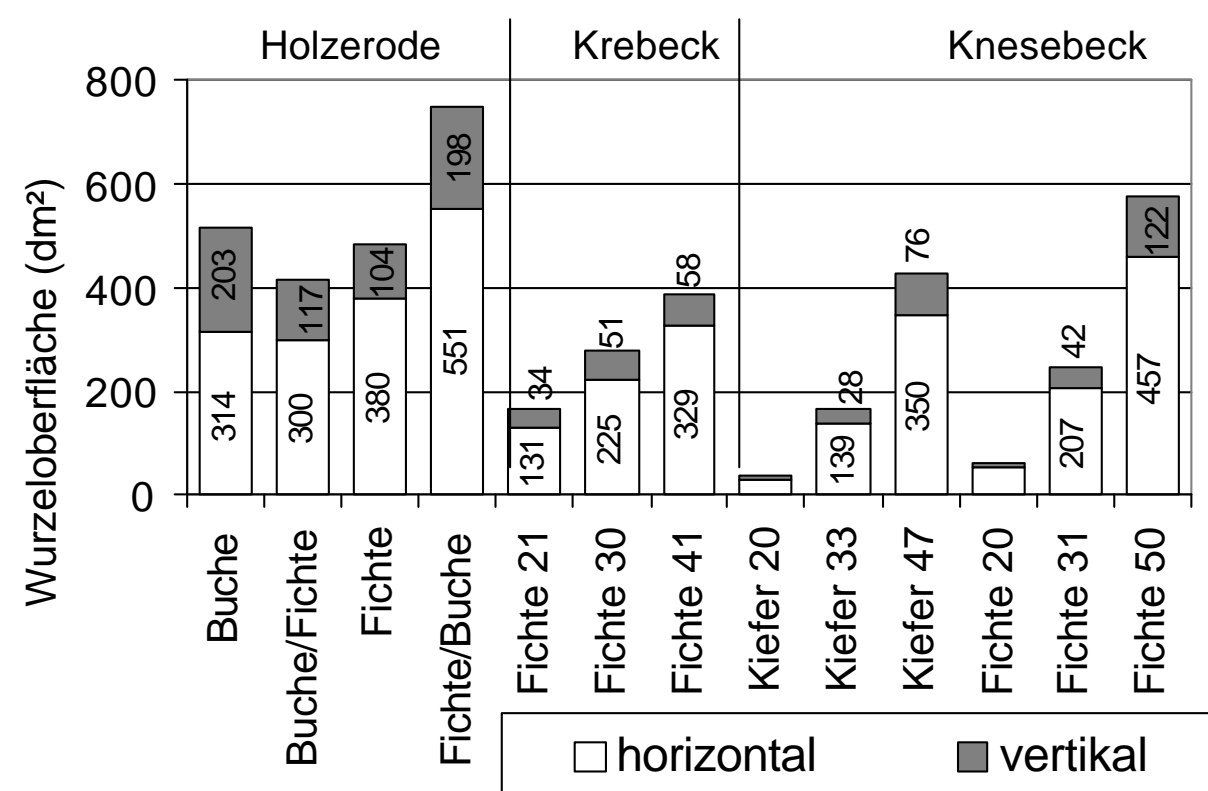

Abb. 26: Oberfläche $\left(d m^{2}\right)$ der horizontalen und vertikalen Wurzeln, Fichte, Buche und Kiefer; Holzerode, Krebeck und Knesebeck

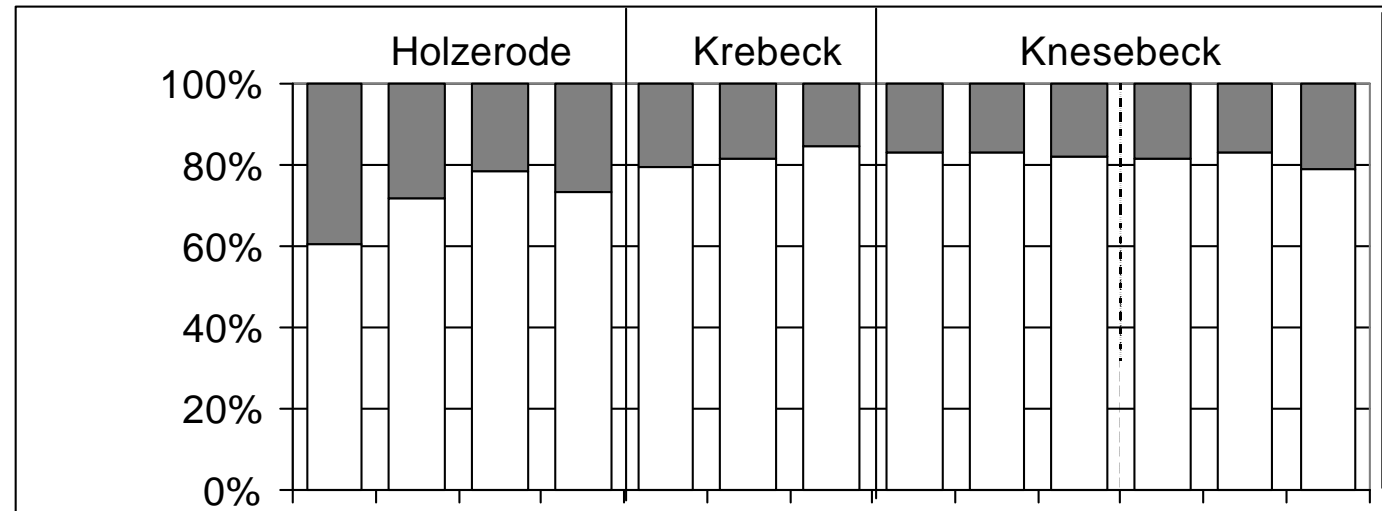

\begin{tabular}{|c|c|c|c|c|c|c|c|c|c|c|}
\hline $\begin{array}{l}\stackrel{0}{\frac{D}{c}} \\
\frac{0}{i} \\
\frac{D}{0} \\
\frac{D}{0}\end{array}$ & $\frac{\frac{0}{T}}{\frac{0}{i}}$ & 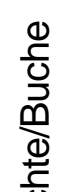 & 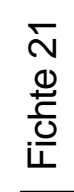 & $\begin{array}{l}\text { ᄋ } \\
0 \\
\frac{0}{1} \\
\frac{0}{0} \\
\frac{0}{4}\end{array}$ & $\begin{array}{l}\bar{y} \\
\stackrel{0}{ \pm} \\
\frac{1}{0} \\
i \frac{0}{4}\end{array}$ & 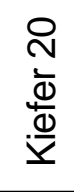 & 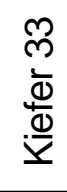 & 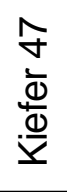 & $\begin{array}{l}\stackrel{O}{0} \\
\frac{0}{ \pm} \\
\frac{1}{0} \\
\frac{0}{4}\end{array}$ & 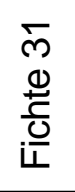 \\
\hline $\bar{\varphi}$ & & & & $\square \mathrm{h}$ & & & & & 10 & \\
\hline
\end{tabular}

Abb. 27: Wurzeloberfläche (\%), differenziert nach Wurzelrichtung (horizontal und vertikal); Fichte, Buche und Kiefer; Holzerode, Krebeck und Knesebeck

\section{Holzminden}

Die Abbildungen 28 und 29 zeigen die Oberfläche der horizontalen und vertikalen Wurzeln der Eichen. Die Saateichen besitzen eine Gesamtwurzeloberfläche von $143 \mathrm{dm}^{2}$ und somit eine $8 \%$ größere Oberfläche als die gepflanzten und eine $28 \%$ größere als die verpflanzten Eichen. Die vertikalen Wurzeln der gepflanzten und der gesäten Eichen haben einen Anteil von rund $20 \%$ an der gesamten Wurzeloberfläche. Bei den verpflanzten Eichen entfallen $40 \%$ der gesamten Wurzeloberfläche auf die vertikalen und $60 \%$ auf die horizontalen Wurzeln. 


\section{Kattenbühl}

In Kattenbühl weisen die Wurzeln der gepflanzten Eichen die größte Oberfläche mit 144 dm$^{2}$ auf (Abbildungen 28 und 29). Die Saat I Eiche besitzt mit $91 \mathrm{dm}^{2}$ eine um $37 \%$ kleinere Wurzeloberfläche. Die Saat II Eiche zeigt eine Wurzeloberfläche von $56 \mathrm{dm}^{2}$. Der Anteil der Vertikalwurzeln steigt von den Pflanzeichen zu den Eichen der Saat II Fläche von $10 \%$ auf $17 \%$ an.

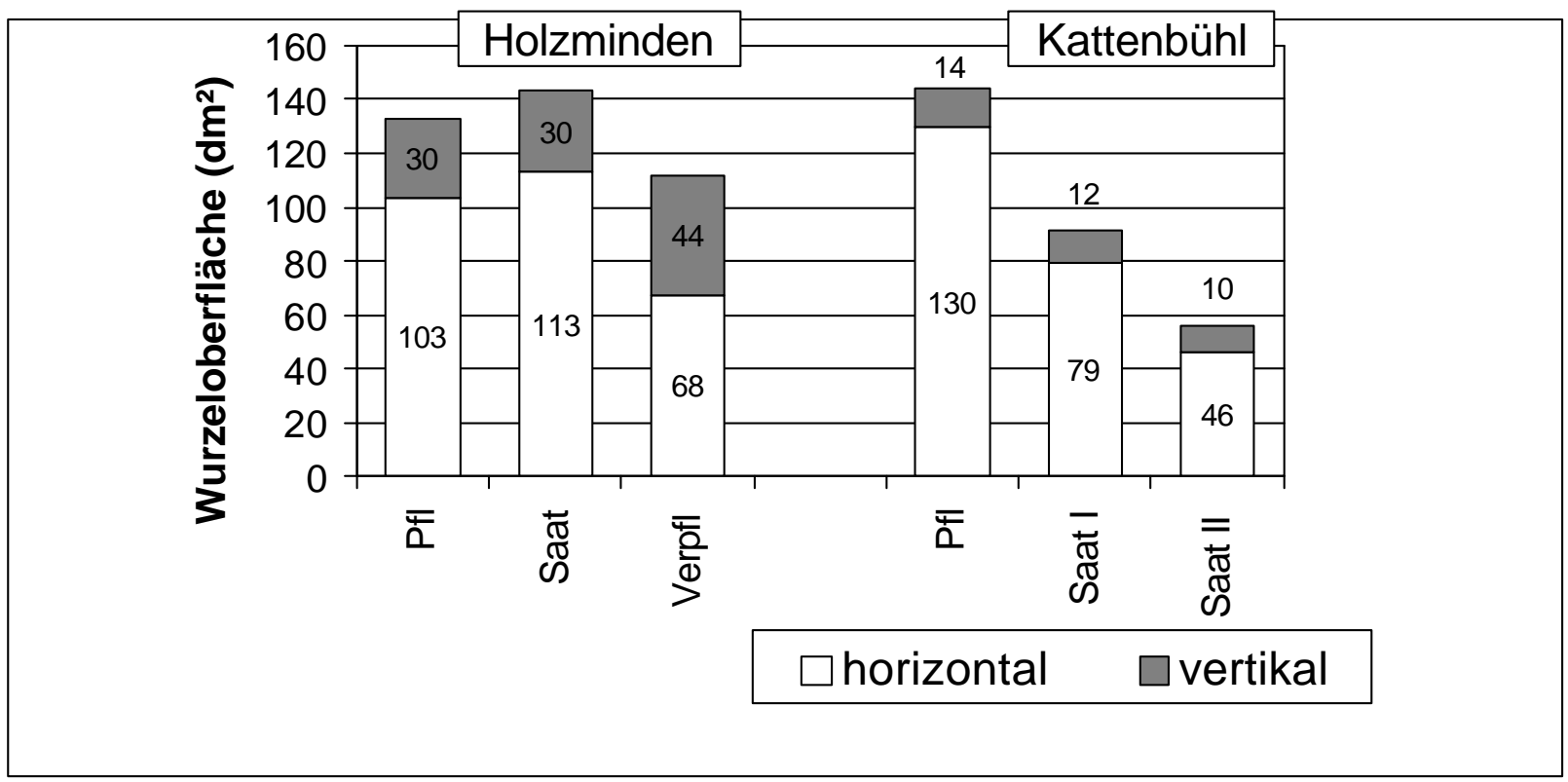

Abb. 28: Absolute Verteilung der Wurzeloberfläche $\left(d m^{2}\right)$ auf horizontale und vertikale Wurzeln; 13- und 22 jährige Eiche, Kattenbühl und Holzminden

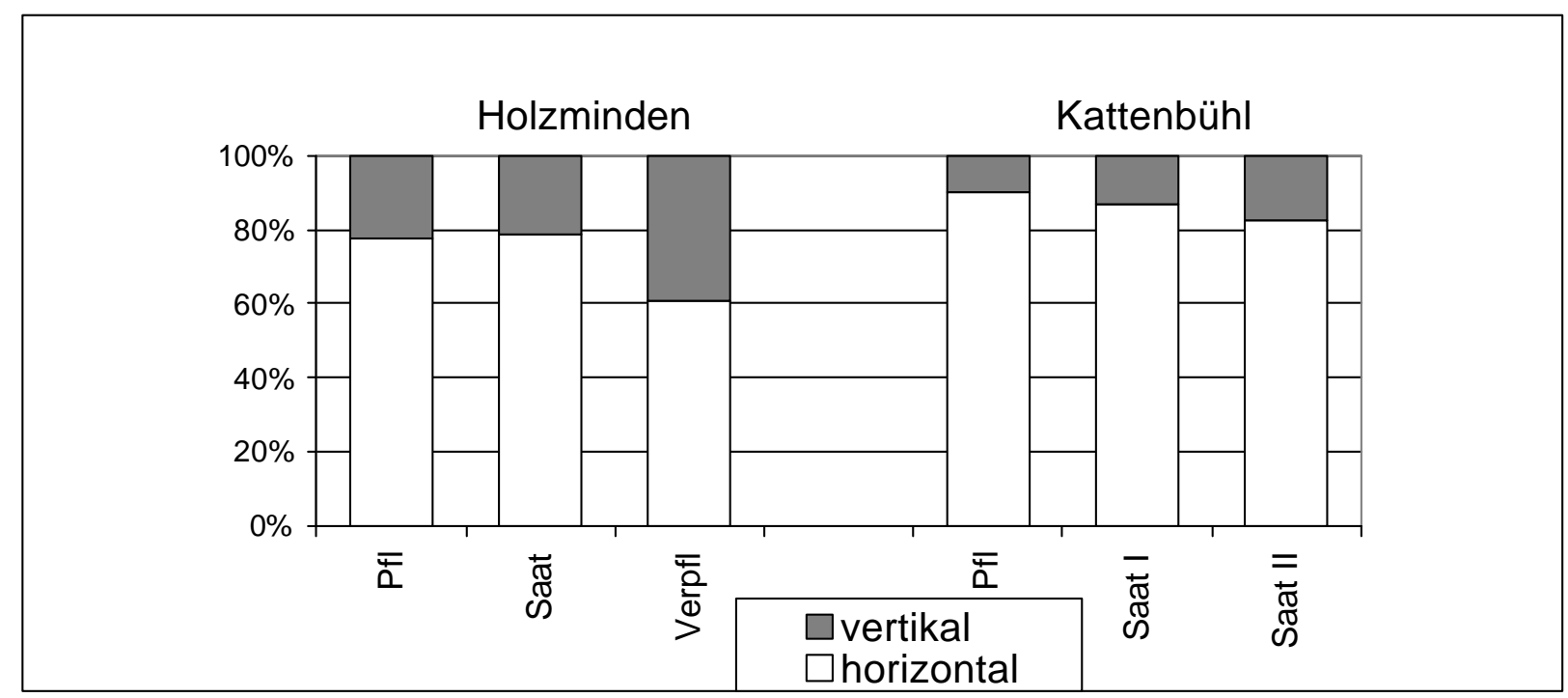

Abb. 29: Prozentuale Verteilung der Wurzeloberfläche auf horizontale und vertikale Wurzeln; 13- und 22 jährige Eichen, Kattenbühl und Holzminden

\subsubsection{Feinwurzelanzahl (FW)}

In Abbildung 30 und 31 sind die Bestandesdurchschnittswerte der Feinwurzeln (FW) dargestellt, die sich an den vertikal und horizontal verlaufenden Grobwurzeln befinden. Tabelle 58 zeigt die Anzahl der Feinwurzeln pro Einzelwurzel. 


\section{Holzerode}

Die Fichten im Reinbestand besitzen durchschnittlich über 1550 Feinwurzeln pro Baum. Damit besitzen sie rund 150 Feinwurzeln mehr als die Buchen im Reinbestand. Beim Vergleich der Mischbestandsflächen weist die Fichte mit insgesamt 650 Feinwurzeln 150 Feinwurzeln mehr auf als die Buche (Abb. 30).

Die Reinbestände weisen an den Horizontal- und Vertikalwurzeln die höchste Feinwurzelmenge auf. Bei der Fichte im Reinbestand ist die Anzahl Feinwurzeln, die sich an den horizontalen Wurzeln befindet, signifikant höher als die der Buchen und Fichten der anderen Flächen (1250 zu 350 und 750). Die Buche im Reinbestand (650 FW) zeigt an den Vertikalwurzeln signifikant höhere Mengen an Feinwurzel als die anderen Bestände (150-300 FW).

Abschließend wird die geschätzte Feinwurzelmenge pro Einzelwurzel des Wurzelsystems (Tabelle 58) betrachtet. Die Mischbestandsbuche besitzt horizontal mit 25 Feinwurzeln signifikant weniger Feinwurzeln pro Wurzel als die anderen Bestände. In der Vertikalen zeigt die reine Buche signifikant höhere Feinwurzeln $(60 \mathrm{FW})$ pro Einzelwurzeln als die gemischte Fichte.

\section{Krebeck}

Die Feinwurzelmenge eines Wurzelsystems der Fichte nimmt vom 21 zum 30 jährigen Bestand um rund $30 \% \mathrm{zu}$ (Abb. 30). Sie steigt von $250 \mathrm{FW}$ auf $300 \mathrm{FW}$ an. Von 30 Jahre bis zum Alter von 41 erhöht sich die Gesamtmenge um $70 \%$. Beim Betrachten der unterschiedlichen Wurzelrichtungen besitzt der 41 jährige Fichte an den Horizontalwurzeln signifikant mehr Feinwurzeln als die Bäume der anderen beiden Flächen. Mit Anstieg des Alters erhöhte sich die Menge von $200 \mathrm{FW}$ auf $450 \mathrm{FW}$. Für die vertikalen Wurzeln liegen die Werte für alle Bestände bei $50 \mathrm{FW}$. Es ist keine Zunahme in Abhängigkeit vom Alter festzustellen.

Die Anzahl der Feinwurzeln pro horizontale Einzelwurzel ist bei den 21 jährigen Fichten um $40 \%$ geringer als bei den 30 jährigen (Tabelle 58). Von 31- zu 41 jährig steigt die Menge auf das Doppelte an. Die absoluten Werte liegen zwischen 15 bzw. 50 Feinwurzeln pro Einzelwurzel. Für die vertikale Wurzel steigt die Feinwurzelanzahl in gleicher Reihenfolge erst gar nicht und dann um das Doppelte. Die 41 jährige Fichte zeigt mit 30 Feinwurzeln eine signifikant höhere Anzahl pro vertikale Wurzel als die jüngere Fichten mit 10 Feinwurzeln.

\section{Knesebeck}

Die Gesamtzahl der Feinwurzeln für ein Wurzelsystem der Fichte steigt von 20 auf 31 Jahre auf nahezu das Dreifache und von 31 auf 50 Jahre um rund $40 \%$ (Abb. 30). Die Fichten haben mit 21 Jahren 150 Feinwurzeln, mit 31 Jahren 400 und mit 50 Jahren 550. Getrennt nach Wurzelrichtung, weisen die Vertikalwurzeln zwischen $50 \%$ und $70 \%$ weniger Feinwurzeln auf als die Horizontalwurzeln.

Die horizontale Einzelwurzel (Tabelle 58) hat bei dem jungen Bestand 10 Feinwurzeln-, die 31 jährige Fichte zeigt nahezu das Dreifache- und die 50 jährige das Vierfache. Die Anzahl der Feinwurzeln der vertikalen Richtung nimmt vom jüngeren $(10 \mathrm{FW})$ zum mittelalten $(15 \mathrm{FW})$ und vom mittelalten zum älteren Bestand (40 FW) um $50 \% \mathrm{zu}$.

Bei den Kiefern (Abb. 30) steigt die Anzahl der Feinwurzeln pro Wurzelsystem mit Zunahme des Alters von 20 Jahre (50 FW) auf 33 Jahre um das Fünffache und von 33 auf 47 Jahre um 
$17 \%$. Dabei unterscheiden sich die Wurzeln der jüngeren Kiefern in ihrer Feinwurzelmenge signifikant von denen der beiden anderen Bestände. Die Differenz zwischen der Feinwurzelanzahl an den Horizontal- und Vertikalwurzeln ist bei den Kiefern schwächer als bei den Fichten. Es sind maximal doppelt soviele Feinwurzeln an den Horizontalwurzeln zu finden wie an den Vertikalwurzeln.

Die Einzelwurzeln der Kiefer (Tabelle 58) zeigen in der Horizontalen $50 \%$ weniger Feinwurzeln als in der Vertikalen. Dabei besitzen die Kiefern abhängig vom Alter zwischen 5 und 20 Feinwurzeln pro horizontale und zwischen 10 und 40 pro vertikale Einzelwurzel. Signifikant weniger Feinwurzeln pro Einzelwurzel zeigt der 20 jährige Bestand gegenüber den anderen Beständen sowohl horizontal als auch vertikal.

Im Vergleich der 30 jährigen Fichten auf Sand (Knesebeck) mit denen auf Lehm (Krebeck) weisen die Wurzelsysteme der ersteren 100 Feinwurzeln mehr auf als die letzteren. Die horizontalen Wurzeln der 30 jährigen Fichten auf Sand haben $16 \%$ mehr Feinwurzeln als die der Fichten auf Lehm. Die Fichte auf Lehm besitzt an den vertikalen Wurzeln halb soviele Feinwurzeln wie die auf Sand.

Die Anzahl der Feinwurzeln, die sich an den horizontalen Einzelwurzeln befinden, unterscheidet sich im Vergleich der beiden Flächen nicht voneinander. An der vertikalen Einzelwurzel zeigt die 31 jährige Fichte auf Sand $25 \%$ mehr Feinwurzeln als die auf Lehm.

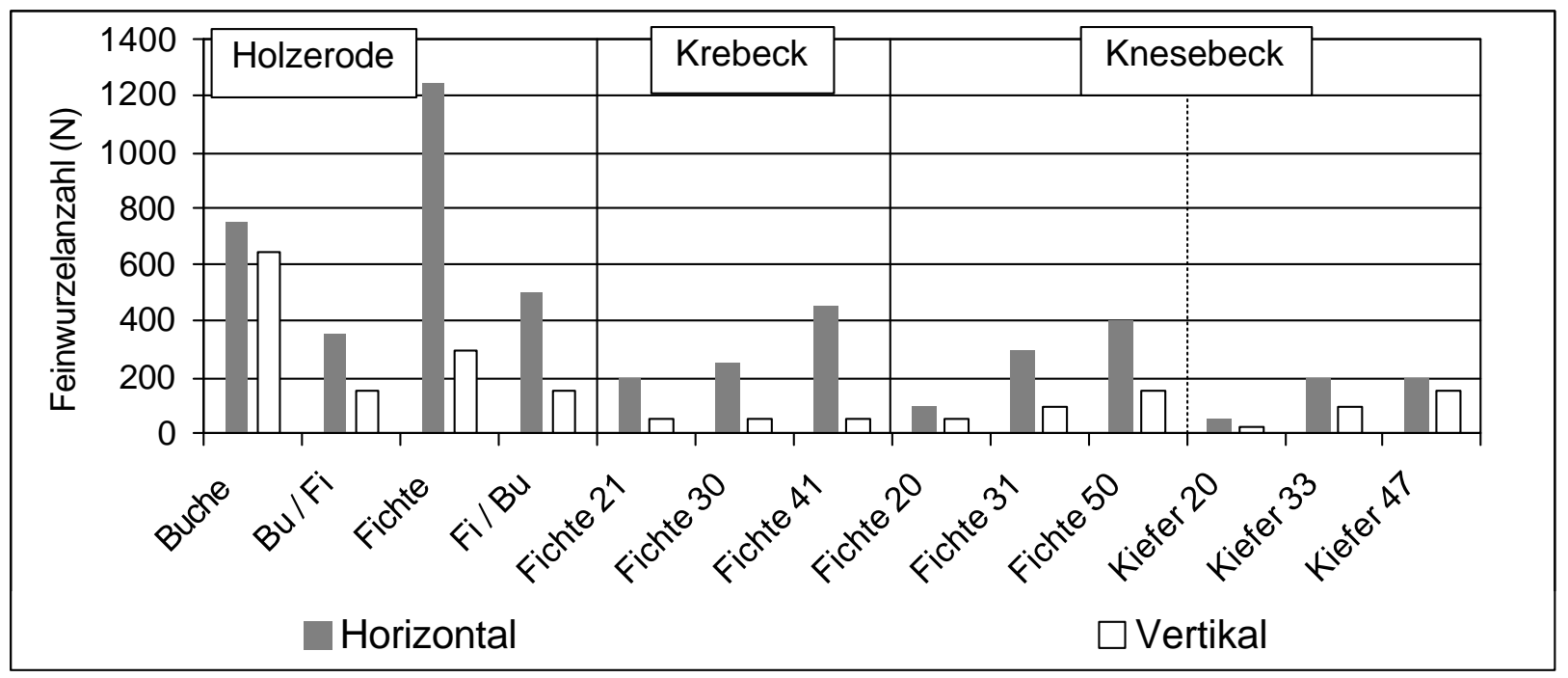

Abb. 30: Geschätzte Anzahl Feinwurzeln pro Wurzelsystem; Holzerode (Fichte und Buche), Krebeck (Fichte) und Knesebeck (Fichte und Kiefer)

\section{Holzminden}

Nach Abb. 31 sind bei den Wurzelsystemen der Saateichen (1350 FW) signifikant höhere Feinwurzelmengen gegenüber den Pflanz- und demVerpflanzungseichen zu finden. Differenziert nach sozialer Stellung, sind bei den herrschenden Saateichen horizontal (1100 FW) signifikant doppelt soviele Feinwurzeln zu finden wie bei den herrschenden Eichen der Pflanzung und der Verpflanzung. An den Vertikalwurzeln sind bei den gepflanzten- und den verpflanzten Bäumen im Vergleich zu den gesäten Eichen (250 FW) etwa halb soviele Feinwurzeln vorhanden. Bei den beherrschten Bäumen zeigt die gesäte Eiche eine doppelt so große (signifikant) horizontale und eine viermal so hohe vertikale Feinwurzelmenge als die gepflanzte. 
Die Mittelwerte der Kraft"schen Klassen 2 und 4 für die Feinwurzeln einer Einzelwurzel sind in Tabelle 58 dargestellt. Dabei zeigt die Horizontalwurzel aller Bestände je rund 25 Feinwurzeln-, die Vertikalwurzel zwischen 15 für die Pflanzung und 40 für die Saat. Differenziert nach Kraft'scher Klasse (Tabelle ist nicht dargestellt) besitzt die Einzelwurzel bei den herrschenden Pflanz- und Saateichen in der Horizontalen 30-, und bei den herrschenden verpflanzten Eichen 20 Feinwurzeln. In der Vertikalen haben die gepflanzten und verpflanzten Eichen 20 Feinwurzeln und die Saateichen 30. Eine durchschnittliche Horizontal- oder Vertikalwurzel der beherrschten gepflanzten Eiche weist 10 auf, die der gesäten besitzt an einer Horizontalwurzel durchschnittlich 20 und an der Vertikalwurzel 40 Feinwurzeln. Die Feinwurzeln, die sich an den vertikalen Wurzeln der gesäten und gepflanzten Eichen befinden, unterscheiden sich signifikant.

\section{Kattenbühl}

Das Wurzelsystem der gepflanzten Eiche zeigt horizontal rund $50 \%$ mehr Feinwurzeln als das der Eiche des Saat I Bestandes (Abb. 31). Letztere besitzt $27 \%$ mehr Feinwurzeln als die Eiche der Saat II Fläche. Vertikal zeigen die Wurzeln der gepflanzten genausoviele Feinwurzeln wie die der Saat II- Eiche und als die Eiche des Saat I Bestandes.

Die gepflanzte Eiche (Tabelle 58) besitzt pro horizontal verlaufende Einzelwurzel 35 Feinwurzeln, in der Vertikalen sind es dagegen nur 20. Sie weist horizontal $17 \%$ mehr Feinwurzeln auf als die Saat I Eiche und die Saat II- Eiche. Für die vertikalen Einzelwurzeln zeigen alle Bestände ähnliche Feinwurzelmengen pro Einzelwurzel.

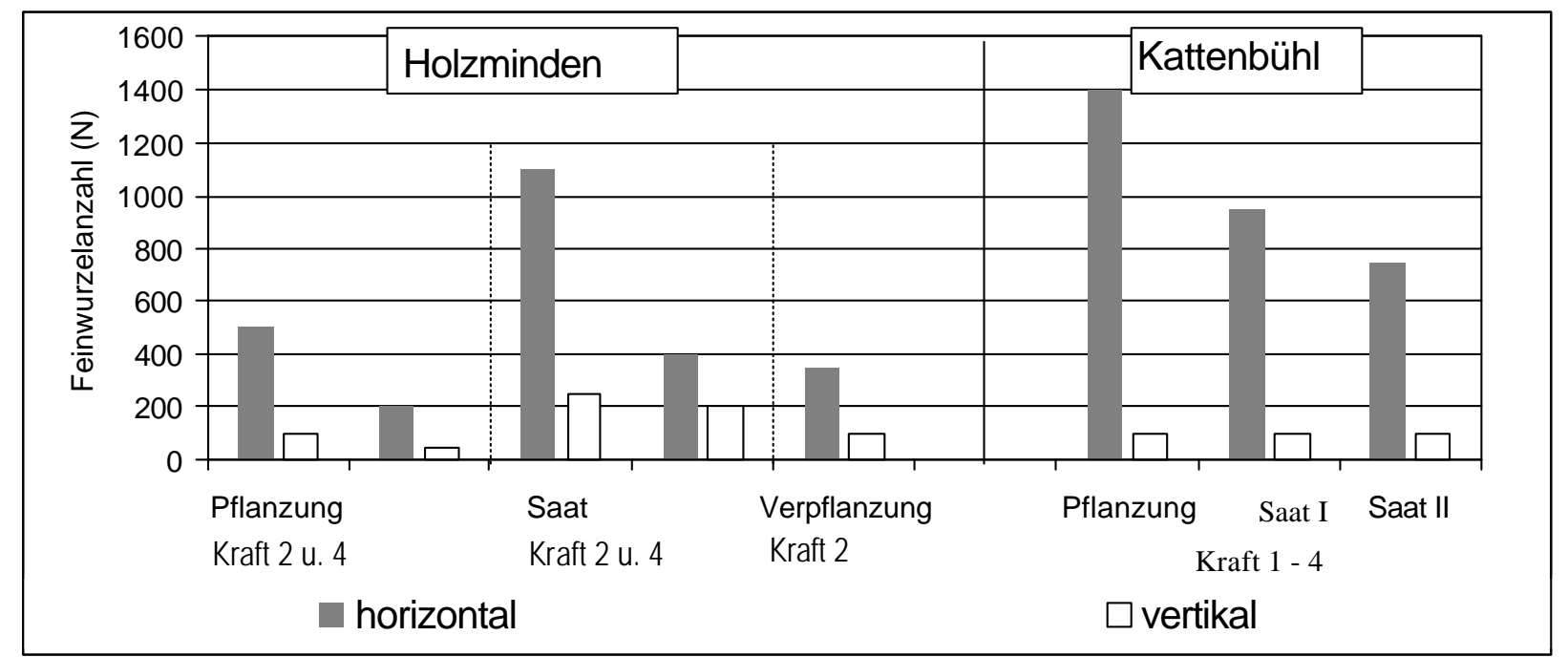

Abb. 31: Mittlere geschätzte Anzahl Feinwurzeln pro Baum, differenziert nach Kraft'schen Klassen; Holzminden, Kattenbühl; 13- und 22 jährige Eiche 
Tabelle 58: Mittlere Anzahl Feinwurzeln und Standardabweichung (sx) pro Einzelwurzel eines Wurzelsystems

\begin{tabular}{|c|c|c|c|c|c|c|c|}
\hline \multirow{5}{*}{$\begin{array}{l}0 \\
\frac{0}{0} \\
\frac{0}{0} \\
\text { 춘 } \\
\text { 오 }\end{array}$} & Bestand & hor (sx) & vert (sx) & \multirow{5}{*}{ 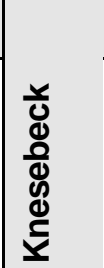 } & Bestand & hor (sx) & vert (sx) \\
\hline & Buche & $60(40)$ & $60(20)$ & & & & \\
\hline & $\mathrm{Bu} / \mathrm{Fi}$ & $25(20)$ & $30(30)$ & & Kiefer 47 & $20(5,84)$ & $40(30)$ \\
\hline & Fichte & $60(20)$ & $30(20)$ & & Kiefer 33 & $10(8,71)$ & $20(20)$ \\
\hline & $\mathrm{Fi} / \mathrm{Bu}$ & $65(30)$ & $35(20)$ & & Kiefer 20 & $5(3,35)$ & $10(8)$ \\
\hline \multirow{3}{*}{ 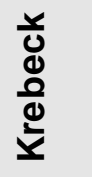 } & Fichte 41 & $50(25)$ & $30(30)$ & \multirow{3}{*}{$\begin{array}{l}\text { 승 } \frac{\mathrm{d}}{0} \\
\text { 오 }\end{array}$} & Pflanzung & $20(11,72)$ & $15(8)$ \\
\hline & Fichte 30 & $25(10)$ & $10(5)$ & & Saat & $25(10,10)$ & $40(25)$ \\
\hline & Fichte 21 & $15(5)$ & $10(8)$ & & Verpflanzung & $25(8,06)$ & $20(5)$ \\
\hline \multirow{3}{*}{ 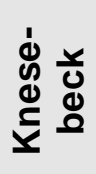 } & Fichte 50 & $40(20)$ & $40(25)$ & \multirow{3}{*}{ 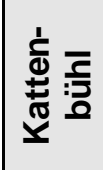 } & Pflanzung & 35 (20) & $20(10)$ \\
\hline & Fichte 31 & $25(15)$ & $15(10)$ & & Saat I & $30(15)$ & $20(10)$ \\
\hline & Fichte 20 & $10(5)$ & $10(5)$ & & Saat II & $30(15)$ & $20(20)$ \\
\hline
\end{tabular}




\subsection{Sproß / Wurzelbeziehungen}

\subsubsection{Beziehungen zwischen dem "HEGYI-Index" und dem Wurzelvolumen}

Der berechnete HEGYI-Index wird nachfolgend für alle untersuchten Probebäume mit dem Wurzelvolumen in Beziehung gesetzt. Die dabei errechneten Funktionen mit den dazu gehörigen Bestimmtheitsmaßen sind in Tabelle 59, differenziert nach Beständen, zusammengestellt. Für die Daten der nicht dargestellten Flächen wurden Regressionen berechnet, deren Bestimmtheitsmaße unter 0,4 liegen. In Abb. 32 wird beispielhaft für den 41 jährigen Fichtenbestand in Krebeck die Beziehung zwischen HEGYI-Index und Wurzelvolumen dargestellt. Die Kurve fällt mit Anstieg des HEGYI-Indexes degressiv ab. Das Wurzelvolumen wird mit zunehmender Nähe und Stärke des Nachbarbaumes überproportional geringer. Ein ähnlicher Kurvenverlauf kann bei allen in Tabelle 59 aufgeführten Beständen festgestellt werden. Bei einem HEGYI-Index von 3,0 nimmt das Wurzelvolumen nahezu für alle Bestände nicht mehr weiter ab. Ausnahmen bilden die Saatbestände der Eiche, bei denen dieser Punkt erst bei einem Index von 4,0 erreicht wird.

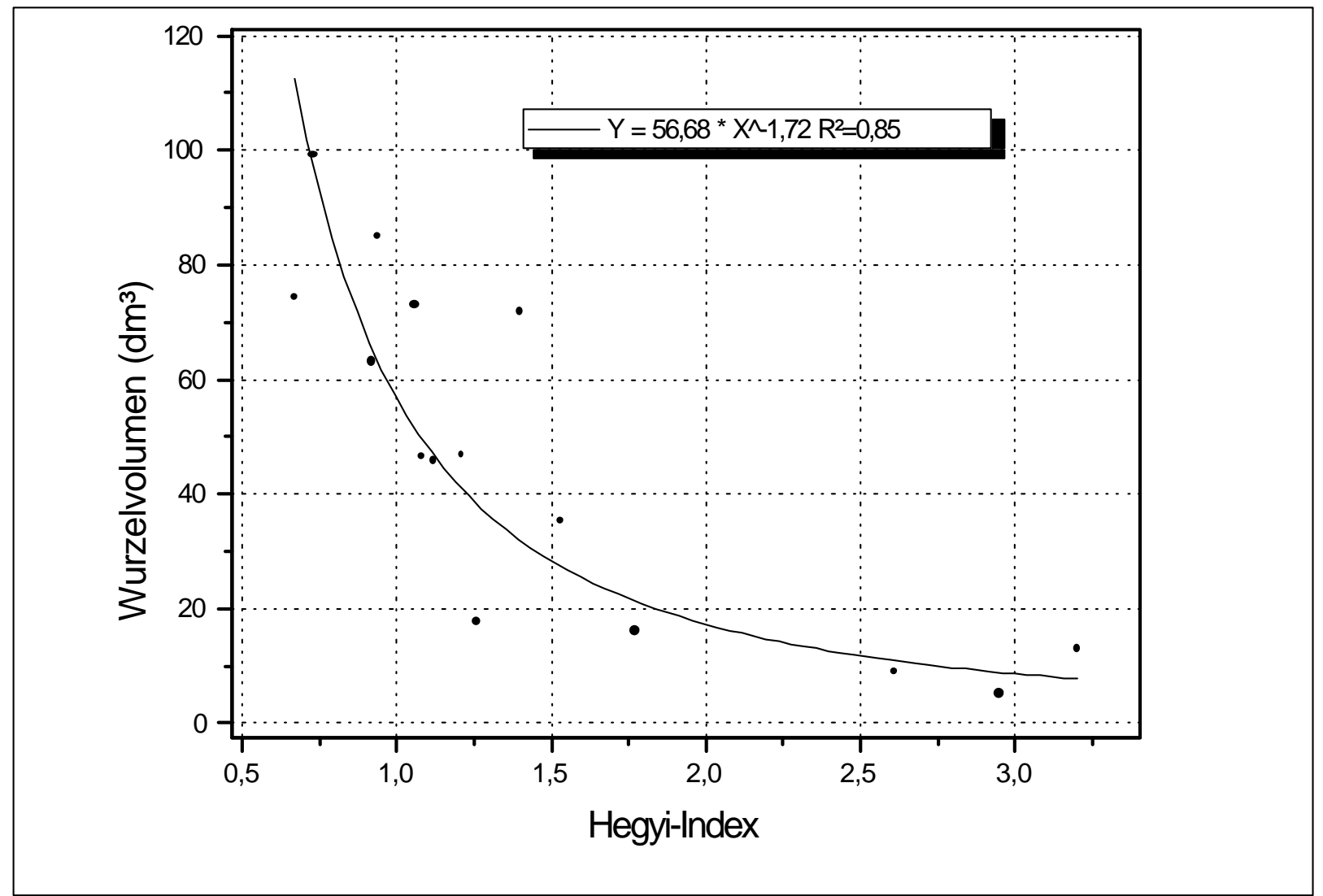

Abb. 32: Regression zwischen dem HEGYI-Index und dem Wurzelvolumen pro Baum am Beispiel eines 41 jährigen Fichtenbestandes 
Tabelle 59: Funktionen der Beziehungen zwischen HEGYI-Index $(X)$ und Wurzelvolumen (Y)

\begin{tabular}{|llc|}
\hline Fläche & \multicolumn{1}{c|}{ Funktion } & $\mathbf{R}^{\mathbf{2}}$ \\
Buche & $\mathrm{Y}=1 /\left(0,008+0,007^{*} \mathrm{X}\right)$ & 0,60 \\
Buche / Fichte & $\mathrm{Y}=1 /\left(-1,65+1,27^{\star} \mathrm{X}\right)$ & 0,41 \\
Fichte / Buche & $\mathrm{Y}=492^{*} \mathrm{EXP}\left(-1,07^{\star} \mathrm{X}\right)$ & 0,83 \\
Fichte 21 & $\mathrm{Y}=19,3-14,2^{*} \mathrm{LOG}(\mathrm{X})$ & 0,73 \\
Fichte 30 & $\mathrm{Y}=62,8^{*} \mathrm{EXP}\left(-0,80^{\star} \mathrm{X}\right)$ & 0,56 \\
Fichte 41 & $\mathrm{Y}=56,7^{*} \mathrm{X}^{\wedge}-1,72$ & 0,85 \\
Kiefer 33 & $\mathrm{Y}=2,09+15,4 / \mathrm{X}$ & 0,82 \\
Fichte 31 & $\mathrm{Y}=-19,16+58,03 / \mathrm{X}$ & 0,67 \\
Fichte 50 & $\mathrm{Y}=55,83^{*}-1,49^{\wedge} \mathrm{B}$ & 0,74 \\
Holzminden Saat & $\mathrm{Y}=-2,71+30,23 / \mathrm{X}$ & 0,56 \\
Kattenbühl Saat II & $\mathrm{Y}=1 /\left(-4,07+1,75^{*} \mathrm{X}\right)$ & 0,79 \\
\hline
\end{tabular}

\subsubsection{Beziehungen zwischen ober- und unterirdischen Parametern}

\subsubsection{Verhältnis Stammvolumen zu Wurzelvolumen}

Abb. 33 stellt die lineare Funktion dar, die die Beziehung zwischen Stammvolumen und Wurzelvolumen $\left(\mathrm{R}^{2}=0,86\right)$ für alle 262 untersuchten Bäume beschreibt.

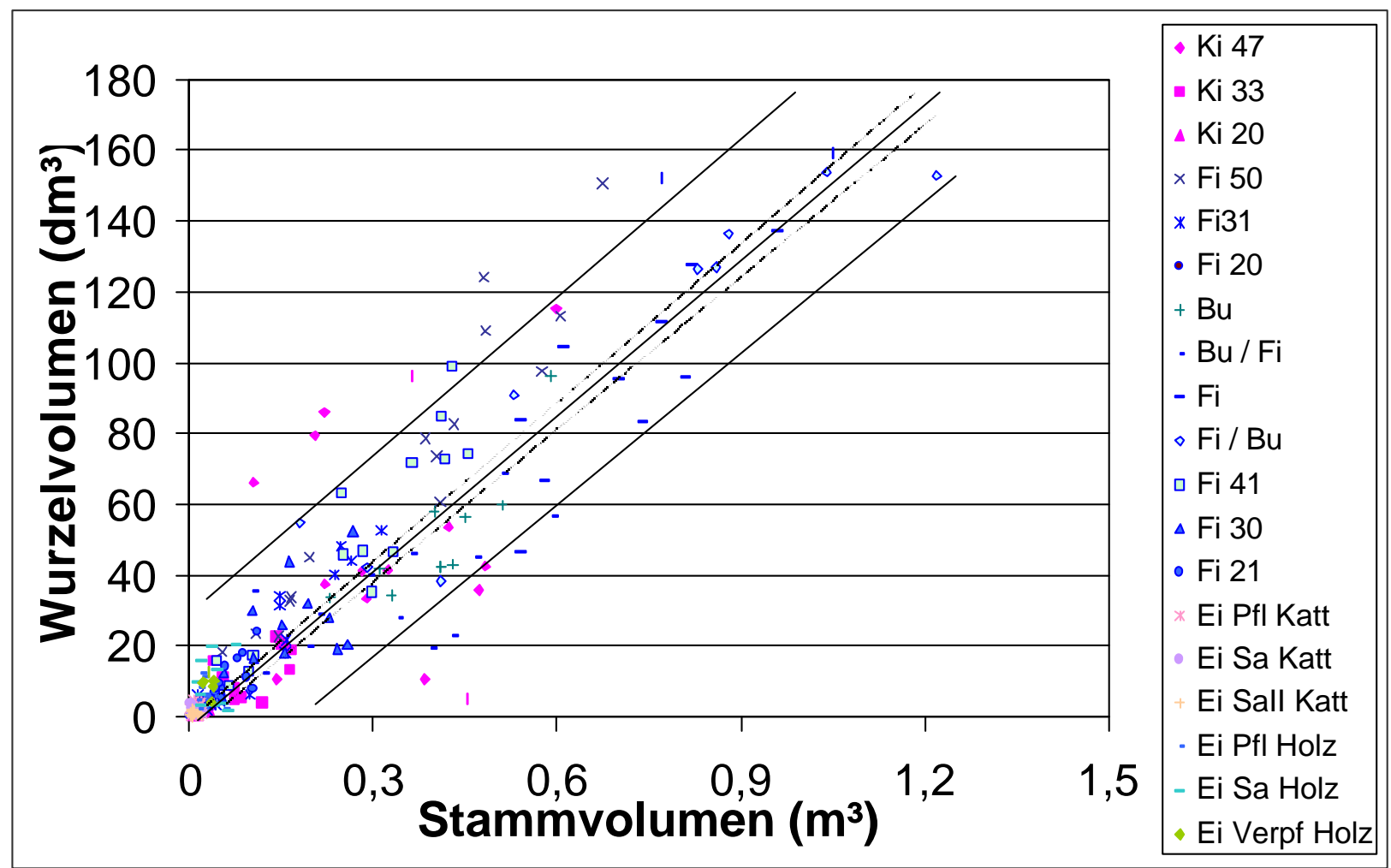

Abb. 33: Beziehung zwischen Wurzelvolumen $\left(\mathrm{dm}^{3}\right)$ und Stammvolumen $\left(\mathrm{m}^{3}\right)$ auf der Grundlage von allen 262 untersuchten Bäumen (Fichte, Buche, Kiefer und Eiche 14 bis 65 jährig), _.... $95 \%$ Vertrauensintervall (Gerade); $95 \%$ Vertrauensintervall (Daten); $R^{2}=0,86 ; y=143,58+1,89 x$ 
Da die Beziehung nicht differenziert nach Baumart und Alter durchgeführt ist, gibt sie nur einen allgemeinen groben Zusammenhang wieder. Die Daten der einzelnen Bestände zeigen keine signifikanten Unterschiede (Kovarianzanalyse mit Test auf Parallelität) zu denen der in Abb. 33 dargestellten Funktion. Um einen tieferen Einblick gewinnen zu können, wird zum einen ermittelt, wie viele Probebäume oberhalb oder unterhalb der in Abb. 33 dargestellten Funktion liegen (Abb. 34 Buche, Fichte, Kiefer, Abb. 35 Eiche). Zum anderen wird mit der Funktion berechnet, welches Wurzelvolumen bei dem real aufgenommenen Stammvolumen der Probebäume vorliegt. Anhand dieses Wertes kann die durchschnittliche prozentuale Abweichung der Bestandesdaten von den durch die Funktion hergeleiteten kalkuliert werden (Abb. 34, Abb. $35)$.

Dabei ergibt sich für drei Buchen des Mischbestandes aus Holzerode eine mittlere prozentuale Abweichung der Punkte, die über der Ausgleichsfunktion liegen, von fast $60 \%$. Unterhalb der Kurve befinden sich die Werte der restlichen neun Probebäume mit einer Differenz von $32 \%$. Dies bedeutet, daß die Streuung des Wurzelvolumens bei der Buche im Mischbestand größer ist als bei der Buche des Reinbestandes.

Bei den Fichten und Kiefern der Flächen aus Krebeck und Knesebeck kann festgestellt werden, daß mit zunehmendem Alter die Anzahl der Bäume steigt, deren Werte oberhalb der Kurve liegen. Dabei nimmt die Streuung der Daten unterhalb der Kurve ab und oberhalb zu. Extrem ist dies für die 47 jährige Kiefer zu beobachten, deren Werte mit einer prozentual hohen Abweichung nach oben und unten variieren. 


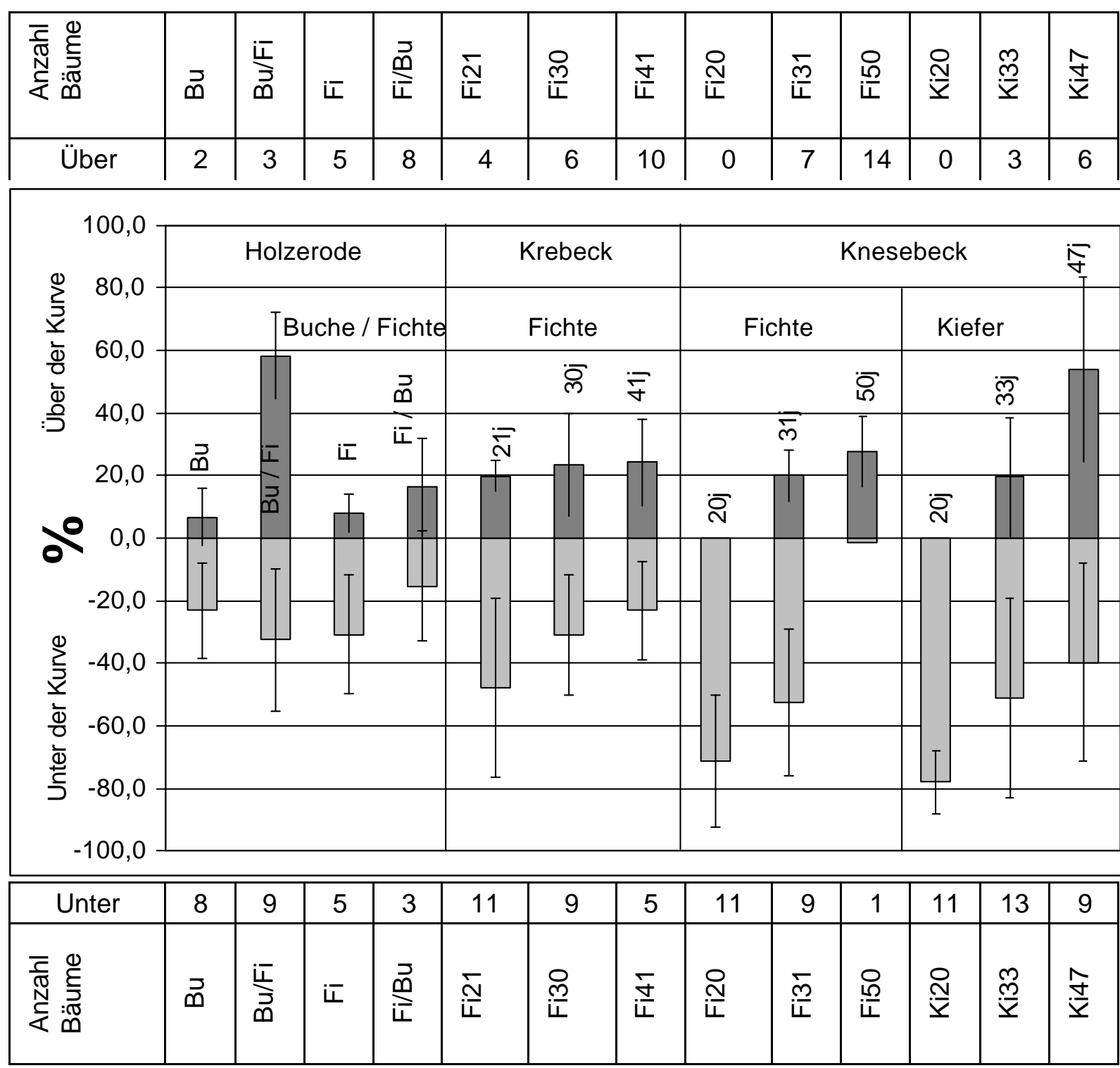

Abb. 34: Durchschnittliche prozentuale Abweichung der Probebäume mit Standardabweichung (Buche, Fichte und Kiefer) von der in Abb. 33 dargestellten Ausgleichsfunktion. Positive Werte beschreiben die Abweichung der Bäume, die oberhalb der Kurve liegen. Negative stellen die Daten der Bäume dar, die sich unterhalb der Kurve befinden. Die Tabellenwerte geben die Anzahl der Bäume wieder, die ober- oder unterhalb der Kurven liegen 


\section{Eiche}

Bei den Eichen des Untersuchungsgebietes aus Holzminden liegen die realen Daten recht gleichmäßig über und unter den nach Abb. 33 errechneten Funktionswerten. Die Probebäume der Eichen aus Kattenbühl zeigen hingegen durchgehend ein Wurzelvolumen, das unterhalb der Kurve liegt. Die jüngeren Bäume haben nach der berechneten Funktion bei gleichem Stammvolumen wie die Eichen aus Holzminden ein geringeres Wurzelvolumen.

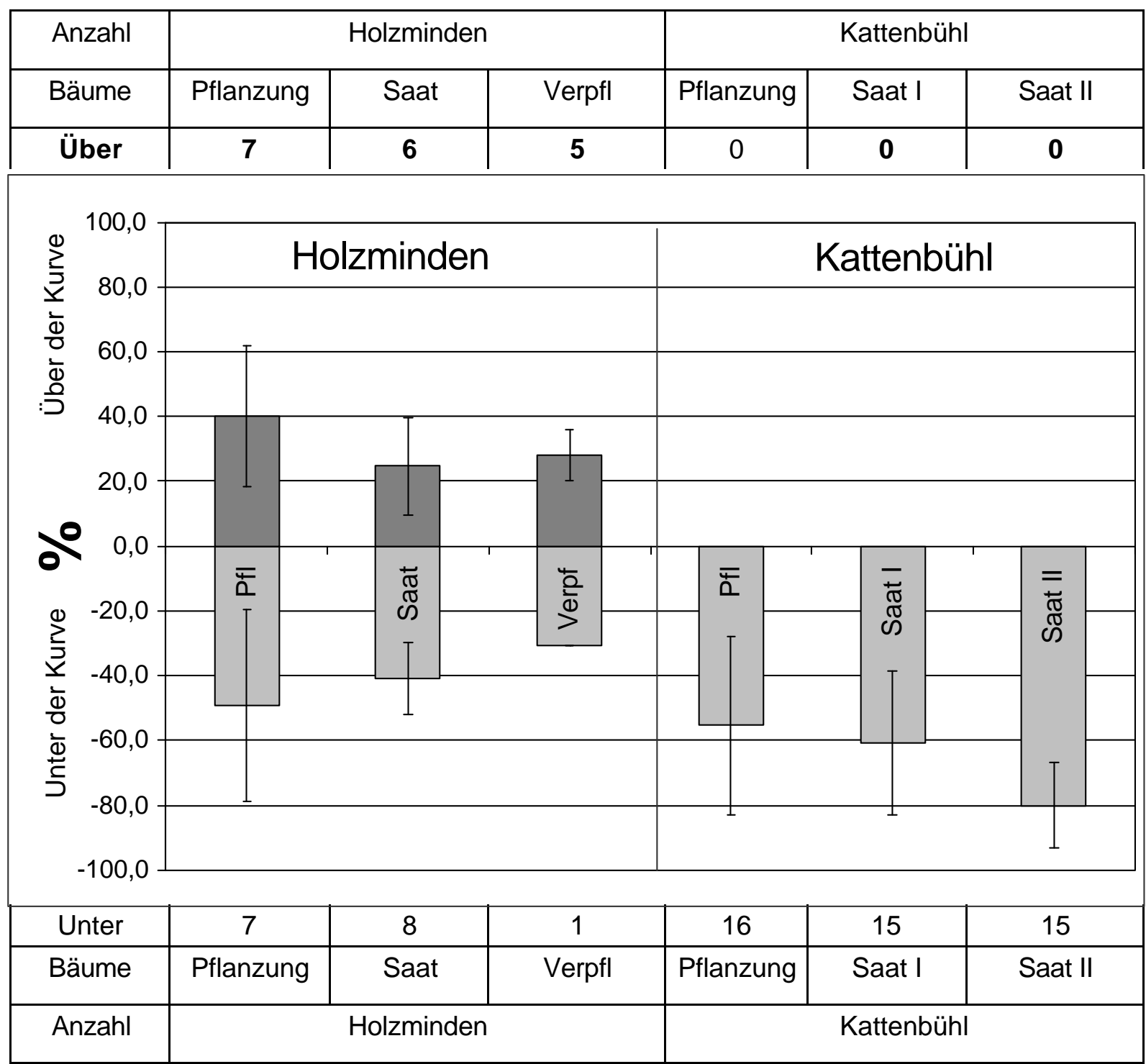

Abb. 35 : Durchschnittliche prozentuale Abweichung der Probebäume (Eiche) von der in Abb. 33 dargestellten Ausgleichsfunktion mit Standardabweichung. Positive Werte beschreiben die Abweichung der Bäume, die oberhalb der Kurve liegen. Negative stellen die Daten der Bäume dar, die sich unterhalb der Kurve befinden. Die Tabellenwerte geben die Anzahl der Bäume wieder, die ober- oder unterhalb der Kurven liegen 


\subsection{Verhältnis Stamm- zu Wurzelvolumen differenziert nach Beständen}

Um weitere, genauere Informationen über die Beziehung zwischen Stamm- und Wurzelvolumen zu erreichen, werden diese Parameter in einem nächsten Schritt, getrennt nach Baumarten und Altersstufen, hergeleitet. Die Relationen sind überwiegend durch Bestimmtheitsmaße von über 0,5 charakterisiert. Davon machen nur die untersuchten Kiefernbestände eine Ausnahme, für die kleinere Bestimmtheitsmaße berechnet werden. Im folgenden sollen die gefundenen Zusammenhänge bei den Fichten, Buchen und Eichen erörtert werden. Die Kiefern werden aufgrund der schlechten Beziehungen nicht beschrieben. Die Funktionen der anderen Bäume sind in Tabelle 60 dargestellt.

Tabelle 60: Parameter der linearen $\left.Y=A+B^{\star} X\right]$ Beziehungen zwischen Wurzel (Y)- und Stammvolumen $(X)$, getrennt nach Baumart; Fichte, Buche, Eiche. Es werden nur Regressionen mit dem Bestimmtheitsmaß $\left(R^{2}\right) \quad 0,5$ dargestellt

\begin{tabular}{|c|c|c|c|c|c|c|c|c|c|}
\hline & Fläche & A & B & $\mathbf{R}^{2}$ & & Fläche & A & B & $\mathbf{R}^{2}$ \\
\hline \multirow{4}{*}{$\begin{array}{l}\frac{0}{0} \\
\frac{0}{0} \\
\text { N } \\
\text { 우 }\end{array}$} & Buche & $-10,16$ & 155,10 & 0,77 & \multirow{7}{*}{ 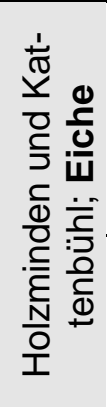 } & & & & \\
\hline & Buche / Fichte & $-1,86$ & 101,75 & 0,64 & & Holz Pflanz. & $-0,02$ & 307,89 & 0,87 \\
\hline & Fichte & $-17,68$ & 159,62 & 0,67 & & Holz Saat & 0,09 & 258,28 & 0,83 \\
\hline & Fichte / Buche & 16,91 & 130,02 & 0,86 & & Holz Verpf. & 0,78 & 281,64 & 0,50 \\
\hline \multirow{3}{*}{ 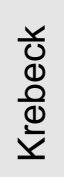 } & Fichte 21 & $-0,81$ & 174,81 & 0,71 & & Katt Pflanz. & 0,15 & 192,12 & 0,79 \\
\hline & Fichte 30 & 4,83 & 119,96 & 0,58 & & Katt Saat I & 0,08 & 194,99 & 0,91 \\
\hline & Fichte 41 & $-1,68$ & 189,05 & 0,64 & & Katt Saat II & 0,19 & 95,798 & 0,62 \\
\hline \multirow{3}{*}{ 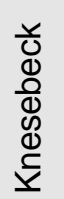 } & Fichte 20 & $-0,29$ & 135,58 & 0,79 & & & & & \\
\hline & Fichte 31 & $-2,74$ & 182,59 & 0,92 & & & & & \\
\hline & Fichte 50 & 0,56 & 200,57 & 0,91 & & & & & \\
\hline
\end{tabular}

\section{Holzerode}

In allen Fällen gibt ein linearer Ausgleich den Zusammenhang zwischen Stamm- und Wurzelvolumen am besten wieder (Abb. 36). Zwischen den verschiedenen Beständen sind keine signifikanten Unterschiede der Ausgleichsfunktionen festzustellen.

Beim Vergleich der untersuchten Bestände weist die Ausgleichsgerade für die gemischte Fichte die größten Funktionswerte auf. Das heißt, diese Bäume besitzen bei einem gleichen Stammvolumen ein höheres Wurzelvolumen als die der anderen Bestände. Dennoch zeigt die Gerade der Fichte im Mischbestand eine geringere Steigung als die des Reinbestandes (Tabelle 60). Dadurch ist bei einem Stammvolumen von $1,17 \mathrm{~m}^{3}$ ein Schnittpunkt vorhanden, ab dem die Fichte im Reinbestand das größere Wurzelvolumen pro Baum besitzt. Die Buchenkollektive zeigen weit auseinanderliegende Geraden. Dabei besitzt die Ausgleichsgerade der Buche im Reinbestand die größte Steigung (Tabelle 60). Bis zu einem Stammvolumen von 0,2 $\mathrm{m}^{3}$ weist die Buche im Mischbestand ein gößeres Wurzelvolumen auf als im Reinbestand. Danach übertrifft das Wurzelvolumen der Reinbestandsbuche das der Mischbestandsbuche. 


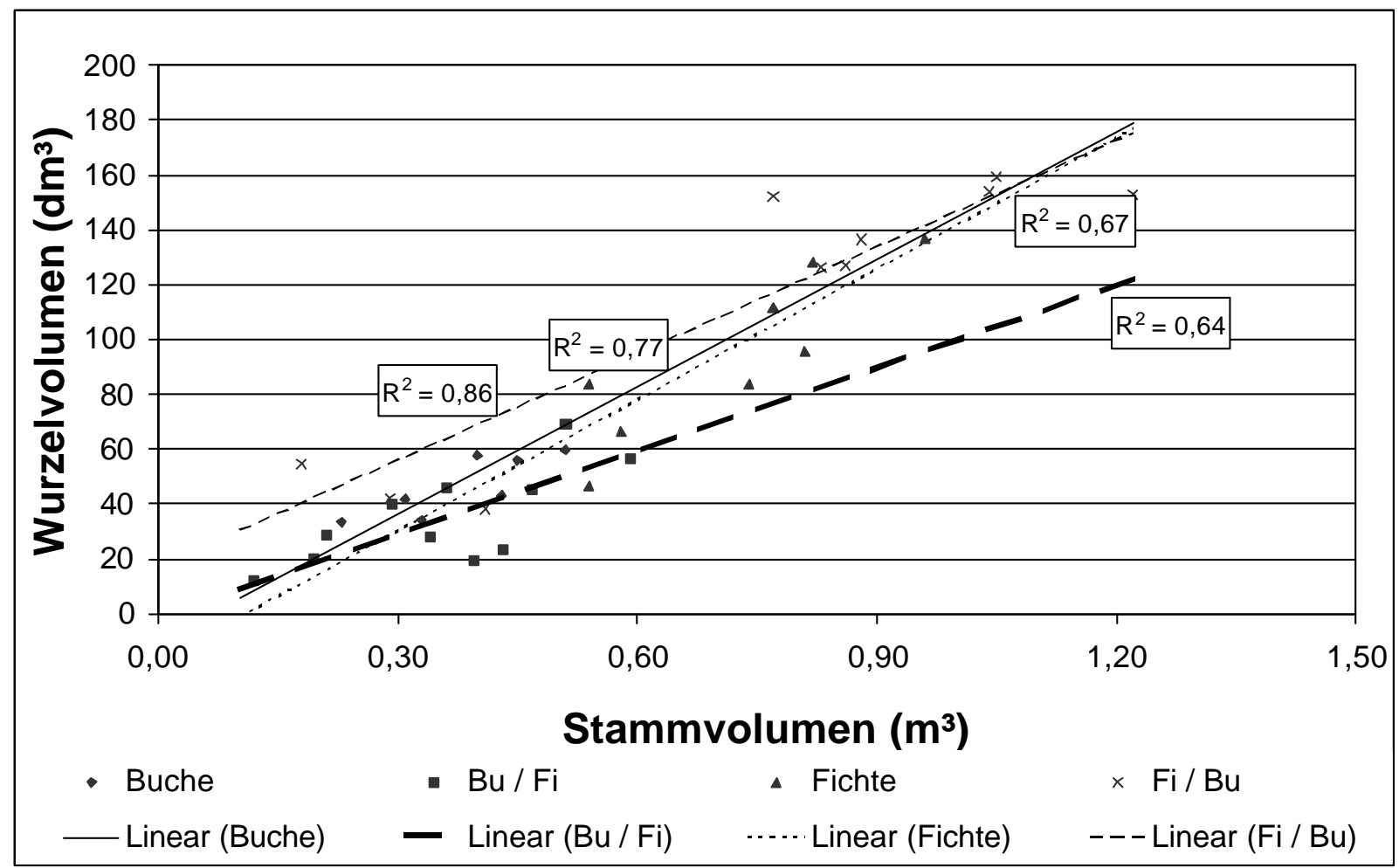

Abb. 36: Regression zwischen Stamm- und Wurzelvolumen; Holzerode; 55-65 jährige Fichte und Buche im Rein- und Mischbestand

\section{Krebeck}

Die linearen Funktionen, die die Beziehungen zwischen Stamm- und Wurzelvolumen beschreiben, sind Tabelle $60 \mathrm{zu}$ entnehmen. Die für die 30 jährigen Fichten ermittelte Ausgleichsgerade weist die geringste Steigung, aber auch das geringste Bestimmtheitsmaß auf (Abb. 37). Dagegen verlaufen die Geraden für die 21- und 41jährigen Bestände fast identisch. Signifikante Unterschiede sind zwischen den drei Beständen nicht festzustellen. Bei einem Stammvolumen von rund $0,10 \mathrm{~m}^{3}$ schneiden sich die lineare Funktionen aller Bestände. Das heißt, das Wurzelvolumen $\left(18 \mathrm{dm}^{3}\right)$ ist an diesem Punkt für alle Bäume gleich groß.

Bei einem beispielhaft gewählten Stammvolumen von $0,06 \mathrm{~m}^{3}$ weist eine 21- oder 41 jährige Fichte ein Wurzelvolumen von $10 \mathrm{dm}^{3}$ auf (Tabelle 61). Die 30 jährige Fichte zeigt bei diesem Stammvolumen ein $20 \%$ höheres Wurzelvolumen. Bei einem Stammvolumen von 0,25 $\mathrm{m}^{3}$ besitzt sie ein Wurzelvolumen von $35 \mathrm{dm}^{3}$ und die ältere Fichte eins von $46 \mathrm{dm}^{3}$. Die junge Fichte weist keine Bäume auf, die ein Stammvolumen von $0,30 \mathrm{~m}^{3}$ erreichen, so daß für diesen Wert kein Wurzelvolumen berechnet wird.

Tabelle 61: Wurzelvolumen bei beispielhaft gewähltem Stammvolumen von 0,06 $\mathrm{m}^{3}$ und 0,25 m³ Krebeck, Fichte 21-, 30- und 41 jährig im Reinbestand (21 jähriger Bestand besitzt nur Bäume mit einem Stammvolumen bis $0,11 \mathrm{~m}^{3}$ )

\begin{tabular}{|ccccccc|}
\hline Stammvolumen & \multicolumn{2}{c}{ Fichte 21 } & \multicolumn{2}{c}{ Fichte 30} & \multicolumn{2}{c|}{ Fichte 41} \\
$\left(\mathrm{~m}^{3}\right) /$ Baum & 0,06 & - & 0,06 & 0,25 & 0,06 & 0,25 \\
Wurzelvolumen $\left(\mathrm{dm}^{3}\right)$ & 10 & - & 12 & 35 & 10 & 46 \\
\hline
\end{tabular}




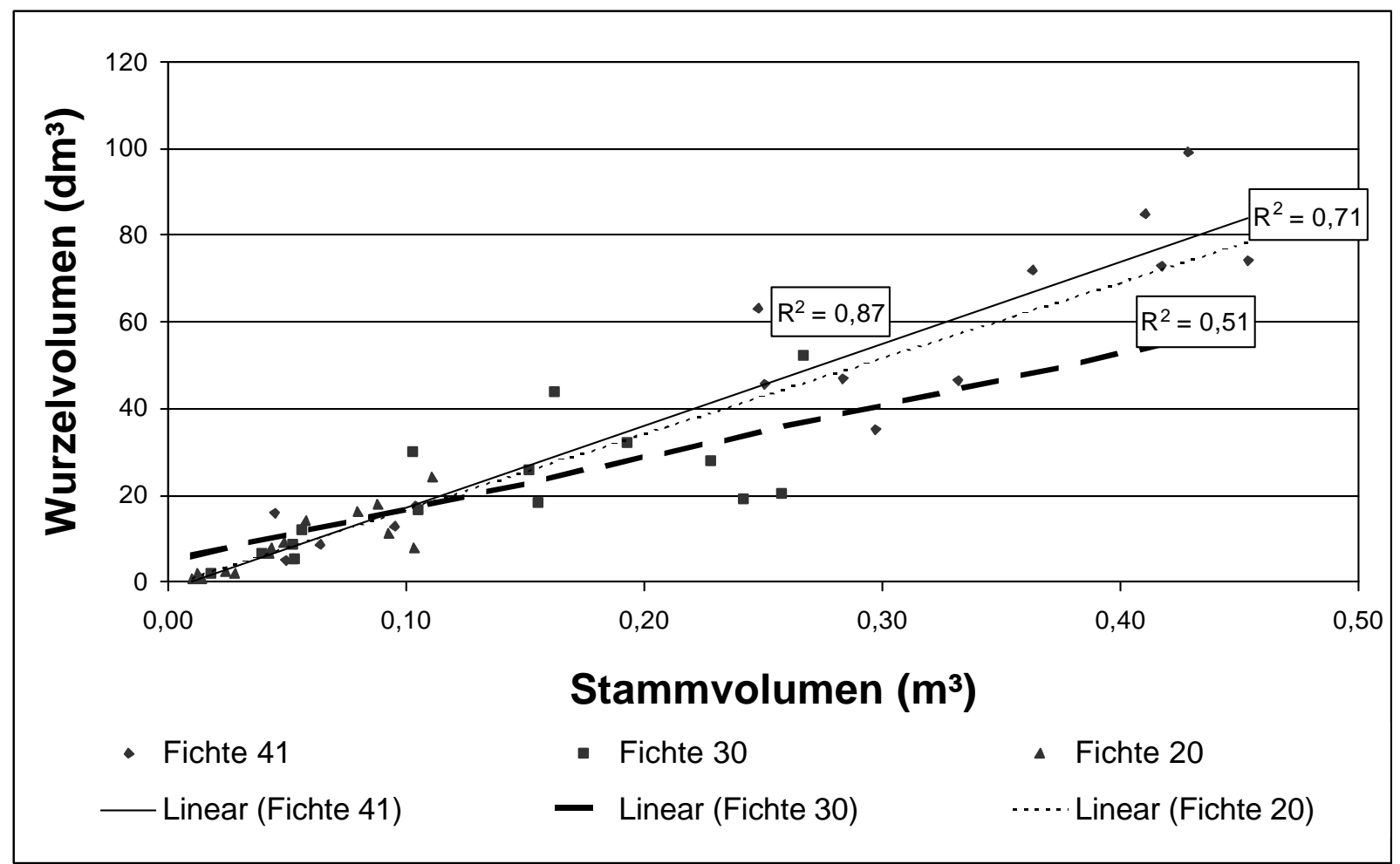

Abb. 37: Regression zwischen Stamm- und Wurzelvolumen; Krebeck, 21-, 30- und 41 jährige Fichte. (Die Geraden der 21 jährigen Fichten enden bei einem Stammvolumen von $0,11 \mathrm{~m}^{3}$ und die des 30 jährigen bei $0,27 \mathrm{~m}^{3}$ )

\section{Knesebeck}

Abb. 38 stellt lineare Zusammenhänge zwischen Stamm- und Wurzelvolumen für die in Knesebeck untersuchten 20-, 31- und 50 jährigen Fichten dar. Die Bäume des jüngsten Bestandes zeigen die geringste Steigung der Geraden (Tabelle 60). Für diese Fläche sind nur Bäume zu finden, die ein Stammvolumen kleiner $0,06 \mathrm{~m}^{3}$ besitzen. Die lineare Funktion der Fichten des 31 jährigen Bestandes zeigt eine geringere Steigung als die der 50 jährigen Bäume. Es sind keine signifikanten Unterschiede der Steigungen der Ausgleichsfunktionen festzustellen.

Beispielhaft wird für ein Stammvolumen von $0,06 \mathrm{~m}^{3}$ das Wurzelvolumen berechnet (Tabelle 62). Dabei zeigt der 20 jährige Fichtenbestand ein Wurzelvolumen von $7,8 \mathrm{dm}^{3}$ pro Baum. Damit besitzen diese Bäume ein $7 \%$ geringeres Wurzelvolumen als die 31 jährigen Fichten. Diese weisen wiederum ein $33 \%$ geringeres Wurzelvolumen auf als die 50 jährigen Fichten $\left(12,6 \mathrm{dm}^{3}\right)$. Bei einem Stammvolumen von $0,3 \mathrm{~m}^{3}$ zeigen die 31 jährigen Bäume ein Wurzelvolumen von $50 \mathrm{dm}^{3}$. Die älteren Fichten haben mit einem Wert von $0,06 \mathrm{~m}^{3}$ ein $20 \%$ höheres Wurzelvolumen als die 31 jährigen.

Tabelle 62: Wurzelvolumen bei beispielhaft gewählten Stammvolumina von 0,06 $\mathrm{m}^{3}$; und 0,3 Knesebeck, 20-, 31- und 50 jährige Fichte

\begin{tabular}{|ccccccc|}
\hline Stammvolumen & \multicolumn{2}{c}{ Fichte 20 } & \multicolumn{2}{c|}{ Fichte 31 } & \multicolumn{2}{c|}{ Fichte 50} \\
$\left(\mathrm{~m}^{3}\right) /$ Baum & 0,06 & - & 0,06 & 0,3 & 0,06 & 0,3 \\
Wurzelvolumen $\left(\mathrm{dm}^{3}\right)$ & 7,8 & - & 8,4 & 50 & 12,6 & 60 \\
\hline
\end{tabular}




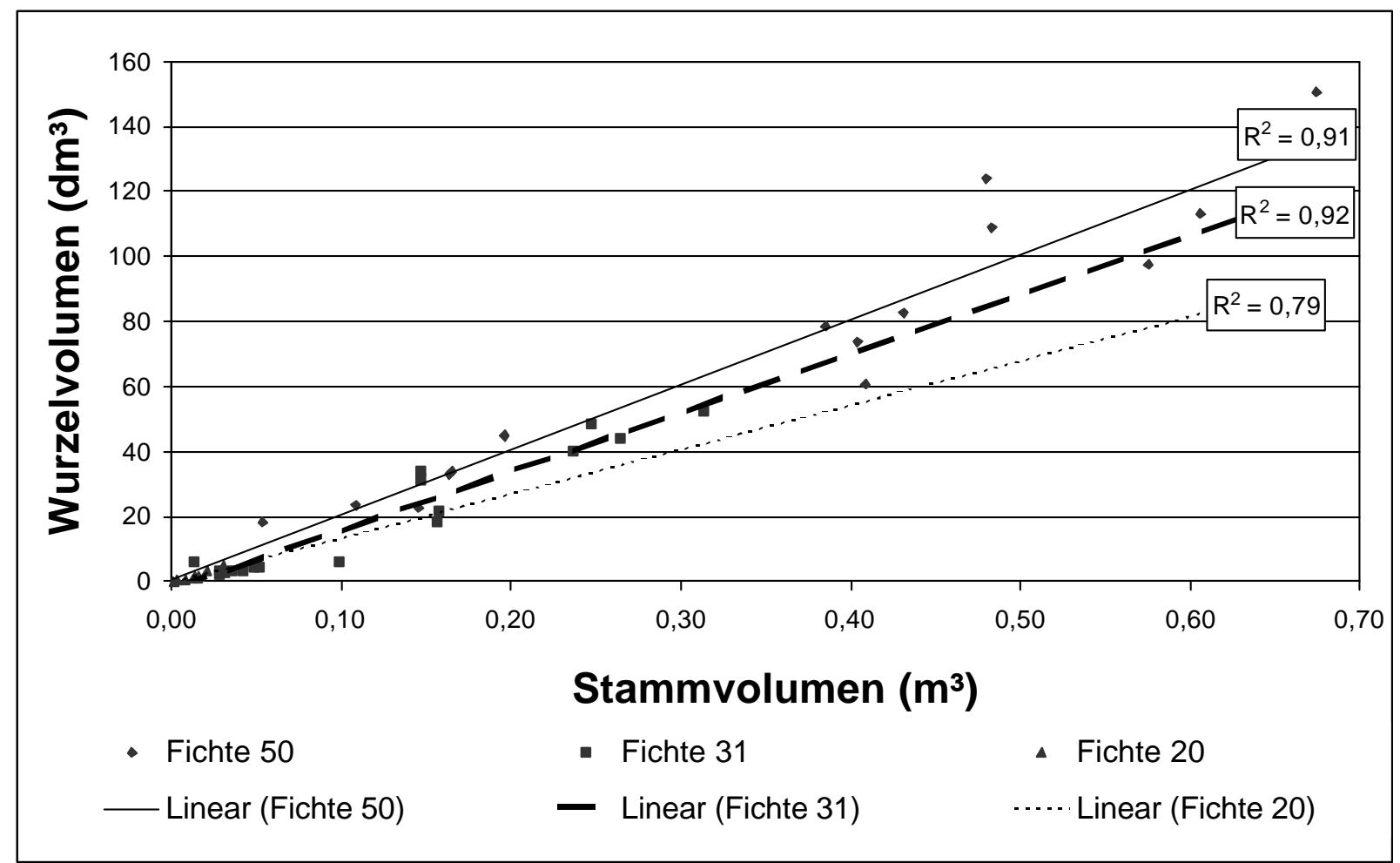

Abb. 38: Regression zwischen Stamm- und Wurzelvolumen; Knesebeck, 20-, 33- und 47 jährige Fichte. (Die Geraden der 21 jährigen Fichten enden bei einem Stammvolumen von $0,06 \mathrm{~m}^{3}$ und die der 30 jährigen bei 0,32 $\mathrm{m}^{3}$ )

\section{Holzminden}

Abb. 39 zeigt die Beziehungen zwischen Stammvolumen und Wurzelvolumen der untersuchten Eichen in Holzminden. Die für sie berechneten Bestimmtheitsmaße erreichen Werte zwischen 0,5 und 0,9 (Tabelle 60). Die lineare Ausgleichsfunktion der verpflanzten Eichen kann den Meßdaten nicht in der Güte angepaßt werden, wie es bei den anderen Flächen der Fall ist. Das Bestimmtheitsmaß liegt hier bei 0,5. Signifikante Unterschiede der Steigung der Ausgleichsfunktionen sind nicht zu finden.

Die Gerade der Pflanzeichen zeigt eine geringere Steigung als die der Saateichen. Die Bäume der Saat besitzen bei gleichem Stammvolumen ein größeres Wurzelvolumen als die der Pflanzung. Bei einem Stammvolumen von z.B. $0,02 \mathrm{~m}^{3}$ ist bei der gepflanzten und der gesäten Eiche ein Wurzelvolumen von $5 \mathrm{dm}^{3}$ und bei der verpflanzten eines von $6 \mathrm{dm}^{3}$ festzustellen. Bei einem Stammvolumen von $0,07 \mathrm{~m}^{3}$ weisen die Bäume der Pflanzung mit $18 \mathrm{dm}^{3}$ ein um $17 \%$ geringeres Wurzelvolumen auf als die der Saat und die der Verpflanzung.

Tabelle 63: Wurzelvolumen bei beispielhaft gewählten Stammvolumina von 0,02 $\mathrm{m}^{3}$ und 0,07 m; Holzminden, 22 jährige Eiche

\begin{tabular}{|c|c|c|c|c|c|c|}
\hline \multirow{2}{*}{$\begin{array}{l}\text { Stammvolumen } \\
\left(\mathrm{m}^{3}\right) / \text { Baum }\end{array}$} & \multicolumn{2}{|c|}{ Pflanzung } & \multicolumn{2}{|c|}{ Saat } & \multicolumn{2}{|c|}{ Verpflanzung } \\
\hline & 0,02 & 0,07 & 0,02 & 0,07 & 0,02 & 0,07 \\
\hline Wurzelvolumen (dm³) & 5 & 18 & 5 & 21 & 6 & 21 \\
\hline
\end{tabular}




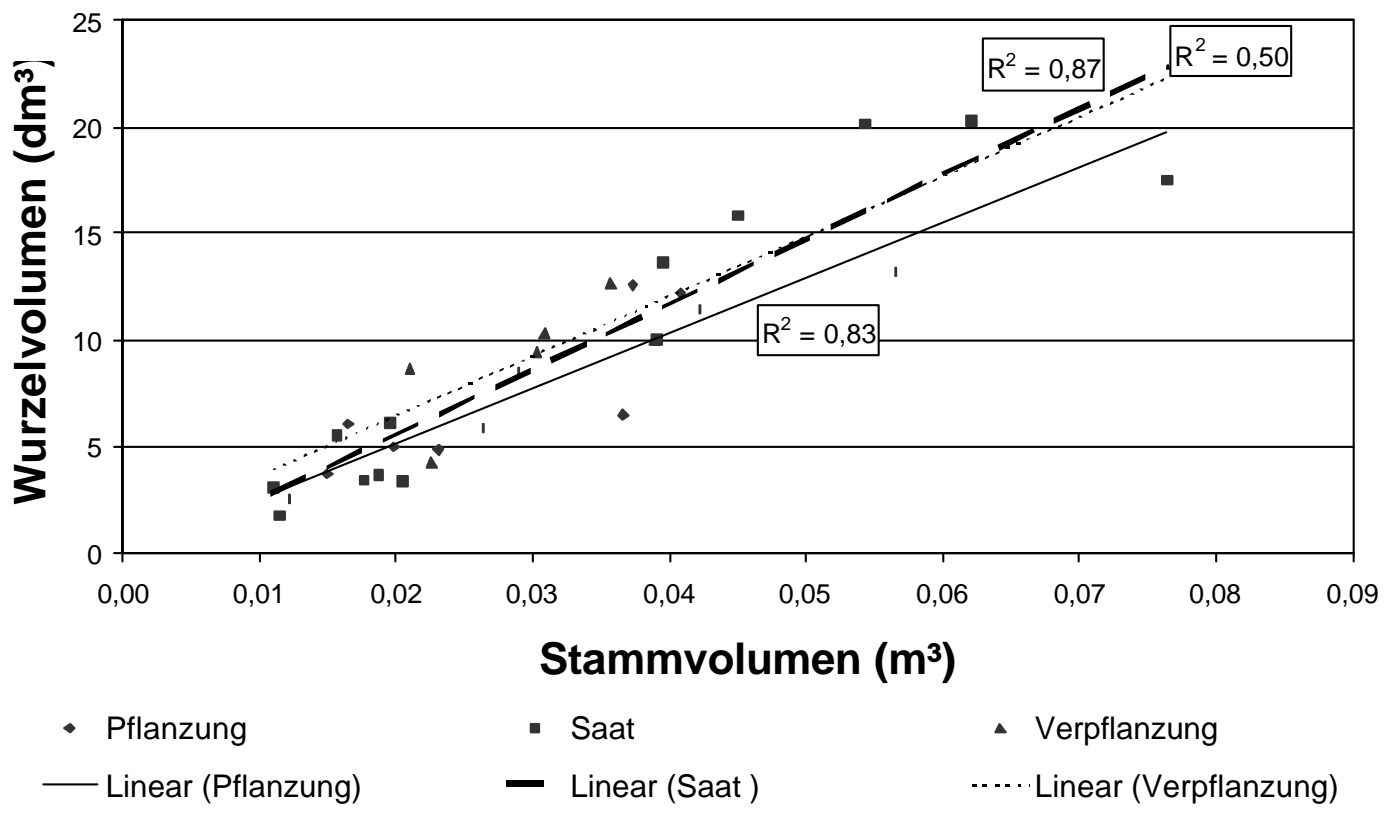

Abb. 39: Regression zwischen Stamm- und Wurzelvolumen; Holzminden, 22 jährige Eiche

\section{Kattenbühl}

Abb. 40 zeigt die linearen Beziehungen zwischen dem Stamm- und dem Wurzelvolumen der 13 jährigen gepflanzten und gesäten Eichen aus Kattenbühl. Tabelle 60 stellt die ermittelten Funktionen dar. Bei Bestimmtheitsmaßen von 0,6 und 0,9 ist von einer guten Anpassung an die Daten auszugehen. Die Geraden der gepflanzten und der gesäten Eichen (Saat I Bestand) weisen sowohl eine nahezu gleiche Steigung als auch ein fast identisches absolutes Glied auf. Die Steigungen der Ausgleichsfunktionen unterscheiden sich nicht signifikant, wohl aber die der Funktionen des Saat I- und Saat des II- Bestandes.

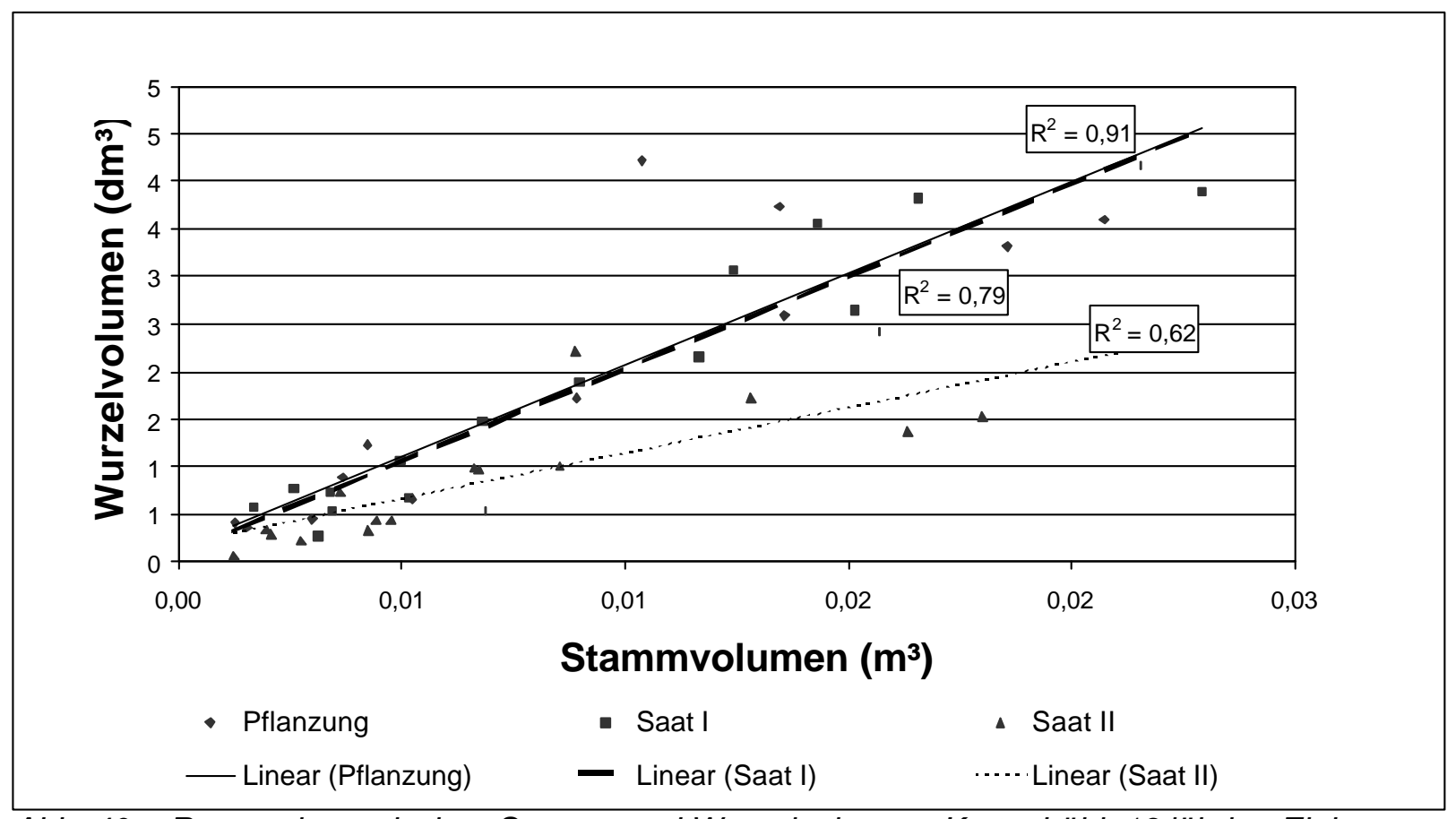

Abb. 40: Regression zwischen Stamm- und Wurzelvolumen; Kattenbühl, 13 jährige Eiche 


\subsubsection{Beziehungen zwischen Wurzel, Stamm und Krone (Flußdiagramm)}

Der Begriff "Flußdiagramm" wird angewendet, um zu verdeutlichen, daß mit einer beliebigen Eingangsgröße, wie z.B. der Höhe oder dem BHD, andere Parameter kalkuliert werden können.

Die in Anhang V, Seiten N-BB dargestellten sechs Grafiken pro Seite zeigen für jeden Bestand das Stamm- und Wurzelvolumen auf der $\mathrm{x}$-Achse und weitere Baumparameter auf der $\mathrm{y}$ Achse. Die Funktionen und Bestimmtheitsmaße sind den Grafiken zu entnehmen. Bestände, deren Regressionen ein Bestimmtheitsmaß unter 0,5 aufweisen, werden nicht dargestellt (Holzerode, Buche; Knesebeck, Kiefer 47 jährig). Bei einem Bestand konnten die Beziehungen zwischen Blattgewicht und Wurzelvolumen nicht hergeleitet werden, da das Blattgewicht nicht ermittelt wurde. Für diese Bäume wird der Kronenansatz zur Bildung der Regression herangezogen (Holzerode, Fichte im Mischbestand).

\section{Holzerode}

In Anhang $\mathrm{V}$, Seite $\mathrm{N}$ und $\mathrm{O}$ werden die linearen Funktionen der ober- und unterirdischen $\mathrm{Pa}$ rameter der Fichten im Rein- und Mischbestand dargestellt. Durchschnittliche Bestimmtheitsmaße von 0,7 geben die gute Anpassung der Regressionen wieder. Für die Buchenbestände sind diese guten Zusammenhänge $\left(\mathrm{R}^{2}<0,5\right)$ nicht $\mathrm{zu}$ finden. Eine Erstellung von Flußdiagrammen wird deshalb nicht vorgenommen.

Im folgenden wird beispielhaft für jeweils eine Fichte des Rein- und des Mischbestandes eine Kalkulation anhand eines standardisierten BHD durchgeführt (Tabelle 64). Mit Hilfe dieser Daten ist ein direkter Vergleich einer Fichte im Rein- und Mischbestand möglich. Als Eingangsgröße wird z.B. ein BHD von $27 \mathrm{~cm}$ gewählt. Dieser entspricht etwa dem mittleren BHD der Probebäume. Durch Funktion (a) aus Tabelle 64 findet als erstes die Berechnung des Stammvolumens für die Fichte des Reinbestandes statt. Mit dem Ergebnis wird anschließend anhand von Funktion (b) die Höhe abgelesen und durch diese wiederum das Kronengewicht (Funktion (c)). Weiterhin wird das Wurzelvolumen und das Blattgewicht kalkuliert (Funktion (d); (e)).

Für die Fichten im Mischbestand sind keine Daten der Blattparameter vorhanden. Um dennoch Informationen zu erhalten, wird statt dessen der Kronenansatz mit dem Stamm- und Wurzelvolumen in Beziehung gesetzt (Tabelle 64). Die Berechnung der Parameter erfolgt entsprechend vorgenannter Tabelle in der dort ausgewiesenen alphabetischen Folge.

Der Vergleich zwischen den berechneten Wurzel-, Stamm- und Kronenparametern der Fichten des Rein- mit denen des Mischbestandes zeigt, daß die Reinbestandsfichte $(27 \mathrm{~m}$ ) bei gleichem Durchmesser $14 \%$ (signifikant) höher ist als die Fichte im Mischbestand. Letztere ist somit abholziger. Das Verhältnis zwischen Stamm- und Wurzelvolumen liegt bei der Fichte im Reinbestand bei 8:1 und bei der im Mischbestand bei 7:1 (nicht signifikant (ns)). Darüber hinaus ist die Krone der Ersteren schwerer (ns) als die der Letzteren. Dies läßt den vorsichtigen Schluß zu, daß das Verhältnis zwischen ober- und unterirdischen Größen bei der Fichte im Reinbestand unausgewogener ist als bei der im Mischbestand. Allerdings unterscheiden sich Kronengewicht, Stamm- und Wurzelvolumen nicht signifikant, so daß der Begriff "vorsichtiges" Ergebnis gewählt wird. 
Tabelle 64: Beispiel einer Berechnung der Parameter eines Baumes anhand der Ausgleichsfunktionen aus Anhang V, Seiten N, 0; Holzerode, Fichte im Rein- und im Mischbestand. Eingangsgröße: BHD $27 \mathrm{~cm}$ (Nadelgewicht war bei der Fichte im Mischbestand nicht verfügbar, so daß an dessen Stelle der Kronenansatz berechnet wird.)

\begin{tabular}{|c|c|c|c|c|}
\hline \multirow{3}{*}{$\mathrm{BHD}(\mathrm{cm})$} & Fichte & \multicolumn{3}{|c|}{ Fichte / Buche } \\
\hline & 27 & \multicolumn{3}{|c|}{27} \\
\hline & Ausgleichsfunktion & Ergebnis & Ausgleichsfunktion & Ergebnis \\
\hline Stammvolumen $\left(\mathrm{m}^{3}\right)$ & (a) $27 \mathrm{~cm}=19,93 \mathrm{x}+13,68$ & 0,67 & (a) $27 \mathrm{~cm}=20,51 \mathrm{x}+12,87$ & 0,69 \\
\hline Höhe (m) & (b) $Y=19,37^{\star} 0,67+14,26$ & 27,27 & (b) $Y=8,29 * 0,69+18,05$ & 23,80 \\
\hline Kronengewicht (kg) & (c) $Y=181,04^{\star} 0,67-7,4$ & 113,90 & (c) $Y=82,4^{\star} 0,69+19,35$ & 76,21 \\
\hline Wurzelvolumen $\left(\mathrm{dm}^{3}\right)$ & (d) $113,90 \mathrm{~kg}=0,74 \mathrm{x}+54,44$ & 80,36 & (d) $76,21=0,53 x+20,17$ & 105,74 \\
\hline Kronenansatz $_{(\mathrm{KA})}(\mathrm{m})$ & & & (e) $y=0,03^{\star} 105,92+20,17$ & 16,25 \\
\hline Nadelgewicht (kg) & (e) $Y=0,48^{*} 80,35+19,04$ & 57,61 & & \\
\hline
\end{tabular}

\section{Krebeck}

Anhang V, P-R, stellt für die Fichtenbestände verschiedenen Alters die allometrischen Beziehungen zwischen Wurzel, Kronen- und Stammparametern dar. Die Daten unterscheiden sich für die verschieden alten Bestände signifikant. Die Funktionen mit den jeweiligen Bestimmtheitsmaßen sind den Grafiken zu entnehmen. Die Bestimmtheitsmaße der errechneten Regressionen zeigen für die 21- und die 41 jährigen Fichten eine sehr gute Anpassung der Funktion mit einem $\mathrm{R}^{2}$ zwischen 0,7 und 0,9. Für die Daten der 30 jährigen Bäume liegen die Werte $\left(\mathrm{R}^{2}=0,5-0,8\right)$ geringfügig niedriger.

Nach dem in Holzerode beschriebenen Schema werden die Wurzel-, Stamm- und Kronenparameter für die 21-, 30- oder 41 jährige Fichte der 1. Ertragsklasse in Krebeck berechnet (Tabelle 65). Diese gewählten Beispieldurchmesser entsprechen in etwa den jeweiligen Grundflächenmittelstämmen. Außerdem werden die Parameter für eine Fichte der drei Altersstufen mit einem BHD von $14 \mathrm{~cm}$ kalkuliert (Tabelle 65). Dieser Wert kennzeichnet einen vorherrschenden bis herrschenden Baum im 21 jährigen Bestand, einen herrschenden bis mitherrschenden im 30 jährigen Bestand und einen bedrängten im 41 jährigen (Abschnitt 4.1.2). Der BHD beträgt für die 21 jährige Fichte der ersten Ertragsklasse bei starker Durchforstung $8 \mathrm{~cm}$, für die 30 jährige $12 \mathrm{~cm}$ und die 41 jährige $17 \mathrm{~cm}$. Die berechneten Parameter Stammvolumen, Höhe und Nadelgewicht steigen vom jungen zum mittelalten Bestand um rund die Hälfte an. Für die ältesten Bäumen ist dies ebenso zu beobachten. Das Kronengewicht (frisch) steigt extremer auf nahezu das Doppelte an. Übertroffen werden diese Werte noch vom Wurzelvolumen. Dieses nimmt von $21 \mathrm{zu} 30$ jährig auf das Neunfache und von $30 \mathrm{zu} 41$ jährig auf fast das Dreifache $\mathrm{zu}$.

Weiterhin werden für alle Flächen die Parameter für je eine 21-, 30- und 41 jährige Fichte mit einem BHD von $14 \mathrm{~cm}$ berechnet. Die Unterschiede der sozialen Klasse werden bei dem Kronengewicht (frisch) besonders deutlich (Tabelle 65). Dieses ist bei der 21 jährigen Fichte am höchsten. Baumhöhe und Stammvolumen steigen bei Alterszunahme um rund $25 \%$ an. Das Wurzelvolumen erhöht sich von $21 \mathrm{zu} 30$ jährig nicht in diesem Maße, wohl aber von 
30 zu 41 jährig. Letzgenanntes besagt, daß die Relation zwischen ober- und unterirdischen Größen mit Abnahme der sozialen Stellung enger wird. Dies wird hervorgerufen durch das geringe Kronengewicht der bedrängten älteren Bäume.

Tabelle 65: Beispiel einer Berechnung von Stamm-, Kronen- und Wurzelparametern anhand der Ausgleichsfunktionen aus Anhang V, P-R; Krebeck, Fichte; Eingangsgrößen: zum einen Fichte, 1. Ertragsklasse, und zum anderen Fichte, BHD $14 \mathrm{~cm}$

\begin{tabular}{|c|c|c|c|c|c|c|}
\hline & \multicolumn{3}{|c|}{$\begin{array}{l}\text { 1. Ertragsklasse (starke Durch- } \\
\text { forstung) }\end{array}$} & \multicolumn{3}{|c|}{ BHD $14 \mathrm{~cm}$ für alle Bestände } \\
\hline & 21 jährig & 30 jährig & 41 jährig & 21 jährig & 30 jährig & 41 jährig \\
\hline BHD (cm) & 8 & 12 & 17 & 14 & 14 & 14 \\
\hline Stammvolumen $\left(\mathrm{m}^{3}\right)$ & 0,02 & 0,08 & 0,22 & 0,09 & 0,12 & 0,14 \\
\hline Höhe (m) & 8,33 & 12,65 & 18,44 & 11,41 & 13,54 & 17,01 \\
\hline Kronengewicht (kg) & 12,47 & 27,82 & 45,21 & 44,44 & 40,14 & 27,88 \\
\hline Wurzelvolumen $\left(\mathrm{dm}^{3}\right)$ & 1,55 & 13,84 & 39,61 & 17,94 & 18,45 & 23,57 \\
\hline Nadelgewicht (kg) & 6,71 & 15,92 & 21,82 & 22,77 & 22,04 & 12,84 \\
\hline
\end{tabular}

\section{Knesebeck}

Anhang V, S-W, stellt die linearen Beziehungen zwischen den Stamm-, Kronen- und Wurzelparametern für die untersuchten Fichten und Kiefern dar. Die jeweiligen Funktionen und Bestimmtheitsmaße $\left(R^{2}=0,5-0,9\right)$ sind denselben Abbildungen zu entnehmen. Für die 47 jährige Kiefer können nur schlecht angepaßte Zusammenhänge zwischen Wurzelvolumen und Kronenparametern gefunden werden. Das Bestimmtheitsmaß liegt unter 0,5.

Um eine Entwicklung in Abhängigkeit des Alters darstellen zu können, wird beispielhaft der BHD des Grundflächenzentralbaumes für die Berechnungen genutzt. Der Wert kann den Bestandesdaten entnommen werden, die in Abschnitt 2.2.3 dargestellt sind. Mit diesem BHD werden die Wurzel-, Stamm- und Kronenparameter berechnet (Tabelle 66).

Dabei hat die 20 jährige Fichte einen BHD von $7 \mathrm{~cm}$, die 31 jährige einen von $13 \mathrm{~cm}$ und die 50 jährige einen von $21 \mathrm{~cm}$. Diese Durchmesserwerte entsprechen in etwa Bäumen einer ersten Ertragsklasse (starke Durchforstung SCHOBER 1975). Das Wurzelvolumen erhöht sich ebenso wie das Stammvolumen von den jungen zu den mittelalten Fichten auf mehr als das Zehnfache und von den mittlelalten zu den ältesten auf etwa das Dreifache. Der Anstieg des BHD und der Höhe sind signifikant abhängig vom Stammvolumen und Alter der Fichten. Darüber hinaus ist der BHD signifikant abhängig vom Wurzelvolumen und Alter des Bestandes.

Ebenso wie für die Fichte des Untersuchungsgebietes in Knesebeck wird für die 20- und 33 jährige Kiefer die Relation zwischen Wurzel-, Kronen- und Stammparametern anhand der Funktionen aus Anhang V, AA-BB, berechnet. Die Ergebnisse werden in Tabelle 66 dargestellt. Für die Kalkulation wird beispielhaft der BHD des Grundflächenzentralbaumes verwendet. Er beträgt für die 20 jährige Kiefer $6 \mathrm{~cm}$ und für die 33 jährige $12 \mathrm{~cm}$. Mit Erhöhung des BHD steigt das Stammvolumen von 21- zu 33 jährig um das Siebenfache (signifikant), die Höhe auf das Doppelte (signifikant) und das Kronengewicht (ns) auf mehr als das Dreifache an (Tabelle 66). Das Wurzelvolumen nimmt von 20 zu 33 jährig um mehr als das Neunfache zu. 
Aus diesen Ergebnissen kann der Schluß gezogen werden, daß die Zunahme der untersuchten Parameter in der Phase von 20- zu 30 Jahren sehr hoch ist. Dieses wird ebenso für die Fichte des Untersuchungsgebietes in Knesebeck festgestellt. Signifikant steigen der BHD und die Höhe der Fichten an. Dies ist abhängig vom Alter, Stamm- und Wurzelvolumen der Bäume.

Tabelle 66: Beispiel einer Berechnung von Stamm-, Kronen- und Wurzelparametern anhand der Ausgleichsfunktionen aus Anhang V, S-W; Knesebeck, Fichte und Kiefer (20-50 jährig); Eingangsgröße BHD des Grundflächenzentralbaumes

\begin{tabular}{|lcccccc|}
\hline & Fichte 20 & Fichte 31 & Fichte 50 & Kiefer 20 & Kiefer 33 \\
BHD $(\mathbf{c m})$ & $\mathbf{7}$ & $\mathbf{1 3}$ & $\mathbf{2 1}$ & $\mathbf{6}$ & $\mathbf{1 2}$ \\
Stammvolumen $\left(\mathrm{m}^{3}\right)$ & 0,016 & 0,11 & 0,32 & 0,01 & 0,08 \\
Höhe $(\mathrm{m})$ & 6,14 & 13,05 & 16,64 & 5,54 & 12,01 \\
Kronengewicht $(\mathrm{kg})$ & 14,96 & 42,18 & 115,84 & 5,09 & 20,05 \\
Wurzelvolumen $\left(\mathrm{dm}^{3}\right)$ & 1,90 & 17,38 & 64,85 & 0,93 & 9,76 \\
Nadelgewicht $(\mathrm{kg})$ & 10,71 & 20,52 & 61,05 & 1,61 & 8,22 \\
\hline
\end{tabular}

\subsection{Vergleich der Wurzel-, Stamm- und Kronenparameter der 30- bzw. 31 jährigen Fichte auf Sand und auf Lehm}

Zum Vergleich der 30- bzw. 31 jährigen Fichten auf den Untersuchungsflächen in Krebeck und in Knesebeck werden im folgenden die Unterschiede der ober- und unterirdischen Parameter anhand der Funktionen aus Anhang V, Q und T, berechnet. Es wird untersucht, inwieweit sich bedingt durch die verschiedenen Bodentypen, Differenzen der Wurzel-, Baum- und Kronenparameter ergeben. Erörtert wird dieses am Beispiel einer Fichte mit dem BHD von $13 \mathrm{~cm}$. Dieser BHD bietet sich für die Berechnung an, da die Ergebnisse für die Fichte auf Sand (Knesebeck) bereits im vorherigen Abschnitt dargestellt werden.

Die Bäume, die auf Lehm stocken, zeigen im Vergleich zu den Fichten auf Sand bei diesem BHD keine signifikanten Unterschiede zwischen den errechneten Parametern. Allein für das Kronengewicht können bei der Fichte auf Sand $22 \%$ höhere Werte festgestellt werden.

Tabelle 67: Wurzel-, Stamm- und Kronenparameter einer 30 bzw. 31 jährigen Fichte auf Lehm und auf Sand bei einem beispielhaft gewählten BHD von $13 \mathrm{~cm}$ (entspricht etwa dem Grundflächenzentralstamm), Berechnung anhand der Funktionan aus Anhang $V, Q$ und $T$

\begin{tabular}{|lcc|}
\hline & Lehm & Sand \\
Bichte 30 & Fichte 31 \\
Stammvolumen $\left(\mathrm{m}^{3}\right)$ & $\mathbf{1 3}$ & $\mathbf{1 3}$ \\
Höhe $(\mathrm{m})$ & 0,10 & 0,11 \\
Kronengewicht $(\mathrm{kg})$ & 13,7 & 14,2 \\
Wurzelvolumen $\left(\mathrm{dm}^{3}\right)$ & 35 & 44 \\
Blattgewicht $(\mathrm{kg})$ & 15 & 17 \\
\hline
\end{tabular}




\section{Holzminden}

Die Flußdiagramme der gepflanzten und gesäten Eichen aus Holzminden sind in Anhang V, XY, dargestellt. Sie stellen die Beziehungen zwischen ober- und unterirdischen Parametern dar. Bestimmtheitsmaße von rund 0,8 geben die gute Anpassung dieser Regressionen für die Saateichen wieder. Bei den Eichen der Pflanzung und Verpflanzung können derartig gute Zusammenhänge nicht immer gefunden werden. Bei den gepflanzten Eichen sind Variationen zwischen Wurzelvolumen und BHD vorhanden. Die Daten der verpflanzten Eichen weisen darüber hinaus auch bei den oberirdischen Größen sehr starke Abweichungen auf. Der Grund hierfür ist wohl die geringe Anzahl der Probebäume, die nur aus einer Kraft'schen Klasse entnommen wurden. Signifikante Unterschiede zwischen den verschiedenen Größen sind zwischen den drei Bestandesformen nicht vorhanden.

Tendenziell kann angemerkt werden, daß Kronengewicht, Stamm- und Wurzelvolumen der Saateiche bei gleichem BHD höher sind als bei den Pflanzeichen.(Tabelle 68). Für die Relation zwischen ober- und unterirdischen Parametern (Stamm- $\left(\mathrm{m}^{3}\right) /$ Wurzelvolumen $\left(\mathrm{m}^{3}\right)$ und Kronengewicht $(\mathrm{kg}) /$ Wurzelvolumen $\left(\mathrm{dm}^{3}\right)$ ) sind nur geringfügige Differenzen zwischen Saat und Pflanzung festzustellen. Dabei weist die Saateiche etwas günstigere Verhältnisse zwischen Stamm $\left(\mathrm{m}^{3}\right)$ und Wurzelvolumen $\left(\mathrm{m}^{3}\right)$ auf als die gepflanzte Eiche. Für die Beziehung zwischen Kronengewicht $(\mathrm{kg})$ und Wurzelvolumen $\left(\mathrm{dm}^{3}\right)$ ist Gegenteiliges festzustellen. Dort zeigt der gepflanzte Baum günstigere Werte als der gesäte, so daß behauptet werden kann, daß sich die Relation zwischen ober- und unterirdischen Parametern ausgleicht.

Tabelle 68: Beispiel einer Berechnung der Parameter einer 22 jährigen Eiche; EingangsgröBen: BHD $7 \mathrm{~cm}$ und $12 \mathrm{~cm}$; Holzminden

\begin{tabular}{|lcrcr|}
\hline & Pflanzung & Saat & Pflanzung & Saat \\
BHD $(\mathbf{c m})$ & $\mathbf{7}$ & $\mathbf{7}$ & $\mathbf{1 2}$ & $\mathbf{1 2}$ \\
\hline Stammvolumen $\left(\mathrm{m}^{3}\right)$ & 0,016 & 0,017 & 0,057 & 0,061 \\
Höhe $(\mathrm{m})$ & 9,7 & 10,8 & 12,6 & 11,9 \\
Kronengewicht $(\mathrm{kg})$ & 4,1 & 6,4 & 26,5 & 30,9 \\
Wurzelvolumen $\left(\mathrm{dm}^{3}\right)$ & 3,9 & 4,7 & 15,2 & 17,5 \\
Blattgewicht $(\mathrm{kg})$ & 1,8 & 0,7 & 8,2 & 3,7 \\
Stamm- / Wurzelvol. $\left(\mathrm{m}^{3}\right)$ & $4: 1$ & $3,6: 1$ & $3,8: 1$ & $3,5: 1$ \\
Kronengewicht $(\mathrm{kg}) /$ & & $1,4: 1$ & $1,7: 1$ & $1,8: 1$ \\
Wurzelvolumen $\left(\mathrm{dm}^{3}\right)$ & $1,1: 1$ & & & \\
\hline
\end{tabular}




\section{Kattenbühl}

Die Grafiken aus Anhang V, Z-BB, stellen die Beziehungen zwischen den ober- und unterirdischen Parametern der in Kattenbühl untersuchten 13 jährigen Eichen dar. Die Ausgleichsfunktionen und Bestimmtheitsmaße sind den Abbildungen zu entnehmen. Zwischen den Beständen sind signifikante Unterschiede für das Kronengewicht in Abhängigkeit vom Stammvolumen und das Blattgewicht (frisch) in Abhängigkeit vom Wurzelvolumen festzustellen. Weiterhin unterscheidet sich das Kronengewicht in bezug zum Wurzelvolumen der Saat II Eichen von den gepflanzten signifikant.

In Tabelle 69 werden Wurzel-, Baum- und Kronenparameter exemplarisch für Bäume mit dem Durchmesser $6 \mathrm{~cm}$ und $8 \mathrm{~cm}$ dargestellt. Es handelt sich nach den in Abschnitt 4.1.2 dargestellten Ergebnissen um eine vorherrschende und eine herrschende Eiche. Dabei sind Kronen- (signifikant), Blatt- (signifikant), Wurzelgewicht und Wurzelvolumen (signifikant) der Eiche des Saat I- Bestandes am geringsten. Daher stellt sich das Verhältnis zwischen dem Stamm- und Wurzelvolumen für die Saat F Eichen am ungünstigsten dar. Die Bäume der anderen Flächen unterscheiden sich diesbezüglich kaum. Die Eichen der drei Flächen unterscheiden sich hinsichtlich der Relation zwischen Kronengewicht und Wurzelvolumen nur geringfügig.

Tabelle 69: Beispiel einer Berechnung der Parameter; 13 jährige Eiche; BHD $6 \mathrm{~cm}$ und $8 \mathrm{~cm}$; Kattenbühl

\begin{tabular}{|c|c|c|c|c|c|c|}
\hline BHD (cm) & $\begin{array}{c}\text { Pflanzung } \\
6\end{array}$ & $\begin{array}{c}\text { Saat I } \\
6\end{array}$ & $\begin{array}{c}\text { Saat II } \\
6\end{array}$ & $\begin{array}{c}\text { Pflanzung } \\
8\end{array}$ & $\begin{array}{c}\text { Saat I } \\
8\end{array}$ & $\begin{array}{c}\text { Saat II } \\
8\end{array}$ \\
\hline Stammvolumen $\left(\mathrm{dm}^{3}\right)$ & 10,3 & 9,9 & 9,9 & 16,9 & 16,1 & 16,4 \\
\hline Höhe (m) & 6,3 & 6,3 & 5,9 & 7,1 & 7,5 & 6,8 \\
\hline Kronengewicht (kg) & 12,1 & 7,6 & 9,7 & 20,5 & 12,0 & 17,2 \\
\hline Wurzelvolumen $\left(\mathrm{dm}^{3}\right)$ & 2,1 & 1,3 & 2,0 & 3,7 & 2,3 & 3,3 \\
\hline Blattgewicht (kg) & 5,2 & 3,0 & 4,06 & 9,1 & 4,9 & 6,8 \\
\hline Wurzelgewicht (kg) & 8,4 & 5,0 & 7,3 & 13,1 & 8,3 & 11,6 \\
\hline $\begin{array}{l}\text { Stamm-/Wurzelvol. } \\
\left(\mathrm{dm}^{3}\right)\end{array}$ & $4,9: 1$ & $7,6: 1$ & $5,0: 1$ & $4,6: 1$ & $7: 1$ & $5,0: 1$ \\
\hline $\begin{array}{l}\text { Kronengewicht }(\mathrm{kg}) / \\
\text { Wurzelvolumen }\left(\mathrm{dm}^{3}\right)\end{array}$ & $1,4: 1$ & $1,5: 1$ & $1,3: 1$ & $1,6: 1$ & $1,5: 1$ & $1,5: 1$ \\
\hline
\end{tabular}




\section{Diskussion}

\subsection{Methode}

Die in dieser Arbeit dargestellten Wurzeldaten sind nur unter Berücksichtigung der Methode und insbesondere deren Qualität zu beurteilen. Nach BöHM (1979) hängt die Methode von dem Ziel der Untersuchung ab. Hierfür ist es notwendig, das bestmöglichst an diesem Ziel orientierte Verfahren zu finden. In Tabelle 70 ist eine Übersicht der Verfahren dargestellt, die in der Literatur zu Untersuchungen der Grobwurzeln genutzt werden (POLOMSKI und KUHN 1998).

Tabelle 70: Übersicht der in der Literatur beschriebenen Methoden für Wurzeluntersuchungen (POLOMSKI und KUHN 1998)

\begin{tabular}{|c|c|c|}
\hline Problemstellung & Methoden & Literatur \\
\hline $\begin{array}{l}\text { Wurzelarchitektur } \\
\text { Vertikale Anordnung }\end{array}$ & $\begin{array}{l}\text { Totalausgrabungen } \\
\text { Teilausgrabungen } \\
\text { Wurzelprofile } \\
\text { Blockmethoden }\end{array}$ & $\begin{array}{l}\text { KÖSTLER et al } 1968 \\
\text { KÖSTLER et al } 1968 \\
\text { KÖSTLER et al } 1968 \\
\text { KARIZUMI 1968, BÖHM und KÖPKE } \\
1977\end{array}$ \\
\hline $\begin{array}{l}\text { Wurzelarchitektur } \\
\text { Horizontale Anordnung }\end{array}$ & $\begin{array}{l}\text { Freilegung mittels Wasserstrahl } \\
\text { Freilegung mittels Vakuumpumpe }\end{array}$ & $\begin{array}{l}\text { KÖSTLER et al 1968; } \\
\text { BÖHM } 1979\end{array}$ \\
\hline $\begin{array}{l}\text { Mechanische Eigenschaften, } \\
\text { Zugfestigkeit }\end{array}$ & Mechanische Belas tbarkeit & $\begin{array}{l}\text { KOKKONEN 1929; WHITELEY } \\
\text { und DEXTER 1984; SCHIECHTLE 1983; } \\
\text { LICHTENEGGER 1985; ABE und IWAMA- } \\
\text { TO 1986; HÄHNE 1991 }\end{array}$ \\
\hline $\begin{array}{l}\text { Wachstumsrate } \\
\text { Lebensdauer } \\
\text { Biomasse }\end{array}$ & $\begin{array}{l}\text { Feldmethoden } \\
\text { 1. Rhizoskop } \\
\text { 2. Glaswand } \\
\text { 3. Bohrkerne } \\
\text { 4. Einwuchsmethode } \\
\text { 5. Profilwand } \\
\text { Labormethoden } \\
\text { 1. Container } \\
\text { 2. Wachstumskammer } \\
\text { 3. Gravimetrische Methoden } \\
\text { 4. Färbemethoden }\end{array}$ & $\begin{array}{l}\text { CARLEY und WATSON 1966; } \\
\text { BÖHM 1979: BROWN und THILENIUS } \\
\text { 1977; PERSSON } 1979 \\
\text { BASSAM und SOMMER 1980; } \\
\text { VOS und GROENWALD 1983; } \\
\text { STEEN 1983; VAVOULIDOU- } \\
\text { THEODOROU und BABEL 1983; } \\
\text { BOSCH 1984: ITOH und BARBER 1983; } \\
\text { SEUFERT et al 1986; BECKER et al 1988 } \\
\text { WÖLLMER 1987; UPCHURCH et al 1988; } \\
\text { FERNANDEZ und PARUELA 1988; } \\
\text { ABERTS Et al 1989; PIJNENBORG et al } \\
\text { 1990; ZOON und TIENDEREN 1990; } \\
\text { CHENG et al 1991; GIJSMAN at al 1991; } \\
\text { EGLI und KÄLIN 1991; WAGNER 1994; } \\
\text { BOX 1996; BRECKLE 1996 }\end{array}$ \\
\hline $\begin{array}{l}\text { Wurzelarchitektur } \\
\text { Verzweigung / Oberfläche }\end{array}$ & $\begin{array}{l}\text { Monolith-Methoden } \\
\text { Box-Methoden } \\
\text { Nadelwand-Methode } \\
\text { Markierte Elemente } \\
\text { Färbemethoden }\end{array}$ & $\begin{array}{l}\text { ELLERN } \text { et al 1970; BÖHM 1979; } \\
\text { STEINBERG und FREYTAG 1980; } \\
\text { STEINBERG und FREYTAG } 1980 \\
\text { SATTELMACHER et al } 1983\end{array}$ \\
\hline
\end{tabular}

Ein Ziel der vorliegenden Arbeit ist es, die Wurzelarchitektur von Fichte, Kiefer, Buche und Eiche schematisiert zu erfassen und zu beschreiben. Die Grobwurzelarchitektur ist als Auf- und Grundriß eines Wurzelsystems zu verstehen. Für diese Zielsetzung wurde die in dieser Arbeit 
durchgeführte Methode der Wurzelaufnahme entwickelt. So war es möglich innerhalb der vorgesehenen Zeit einen großen Stichprobenumfang zu untersuchen und damit waldbauliche Fragestellungen zu bearbeiten.

Das Verfahren kann als eine Mischung der von HILF 1927 durchgeführten Totalausgrabungen und der von ELLERN et al (1970) und BÖHM (1979) beschriebenen Monolith - Methode angesehen werden. Die Wurzelsysteme werden in ihrer Gesamtheit aus der Erde entnommen. Ein dreidimensionales Raster, dessen Aussehen "Tortenstücken" ähnelt, wird über das System gelegt, und an den Durchtrittsstellen werden die Durchmesser der Einzelwurzeln aufgenommen. Es wird demnach bis zu einem Meter Entfernung vom Wurzelstock ein runder Wurzelmonolith entnommen, der in 27 Abschnitte zerteilt und vermessen wird. Nachteil dieser Methode ist, daß durch die Entnahme des Baumes Wurzelabrisse und Veränderungen der Wurzellage, insbesondere der dünneren Wurzeln stattfinden. Dieses Problem kann durch die erstmals von WEAVER (1919) angewandte Profilwandmethode beseitigt werden. Bei diesem Verfahren werden in bestimmten Radien bis in festgelegte Tiefen Grabungen durchgeführt. An deren baumzugewandten Schnittseiten können manuelle Messungen der Wurzeldurchmesser und -Positionen durchgeführt werden oder fotografische Auswertungen mit Hilfe einer computergesteuerten Bildanalyse stattfinden (SUTTON 1978). Um eine Vergleichbarkeit mit den hier durchgeführten Daten zu erlangen, müssen für einen Baum sechs Profile angelegt werden. Dies ist notwendig, um die drei horizontalen und drei vertikalen Radien in verschiedenen Entfernungen vom Stamm zu untersuchen. Die Profilwandmethode ist eine sehr genaue Aufnahmetechnik, für die jedoch ein hoher Zeitaufwand erforderlich ist. In der Literatur sind wenig Zeitangaben für die jeweiligen Methoden dargestellt. BIBELRIETHER (1962) führt für die Erfassung eines freigelegten Wurzelsystems von ca. $20 \mathrm{~cm}$ dicken Eichen durchschnittlich sechs bis acht Stunden Arbeitszeit an. Die für die vorliegende Arbeit durchgeführten Untersuchungen an etwa $10 \mathrm{~cm}$ dicken Eichen dauerten im Schnitt eine Stunde (zwei Personen). Der Arbeitsaufwand ist von vielen Faktoren wie der Bodenbeschaffenheit, dem Alter der Bäume (bzw. BHD), der Baumart und dem Genauigkeitsanspruch der Aufnahmen abhängig und so einer großen Variabilität unterlegen.

Vorteilhaft bei der in dieser Arbeit angewandte Methode ist der im Vergleich zu den anderen Verfahren geringere Zeitaufwand, der umfangreichere Stichproben ermöglicht. Eine Schwierigkeit dieses Verfahrens ist die Variationsbreite bei der Festlegung der Radiengröße, die abhängig sein sollte von der Baumart, dem Alter (BHD) und dem Standort. In der Literatur werden verschieden große Radien angegeben. Dabei lassen sich keine Gesetzmäßigkeiten erkennen. So wählen DREXHAGE (1994) und Do-HyUNG LEE (1998) bei ca. 40jährigen Fichten horizontale Radien von $20 \mathrm{~cm}$ und $50 \mathrm{~cm}$ und vertikale Tiefenstufen von $10 \mathrm{~cm}$ bzw. $20 \mathrm{~cm}$. NIELSEN (1990) mißt bei denselben Baumarten horizontal sowie vertikal in $40 \mathrm{~cm}$ und $100 \mathrm{~cm}$ Entfernung von der Stammbasis. KREUTZER (1961) beschränkt seine Messungen an jüngeren Bäumen auf einen Horizontalradius von $57 \mathrm{~cm}$, in dem er sechs Tiefenstufen, beginnend bei $30 \mathrm{~cm}$, unterscheidet. In der vorliegenden Arbeit werden für die älteren Bäume horizontale und vertikale Radien von $30 \mathrm{~cm}, 60 \mathrm{~cm}$ und $100 \mathrm{~cm}$ und für die jüngeren $20 \mathrm{~cm}-, 40 \mathrm{~cm}-$ und $70 \mathrm{~cm}$ - Radien genutzt. Tabelle 71 gibt einen Überblick über die verwendeten Radien in Abhängigkeit vom BHD der Probebäume. Für eine genauere Vermessung empfiehlt es sich, weitere Sektionen in gleichmäßigen Abständen in die vorgeschlagenen Distanzen einzubinden. 
Diese sollten zum Beispiel bei einem BHD von $16 \mathrm{~cm}$ bis $30 \mathrm{~cm}$ in $15 \mathrm{~cm}, 45 \mathrm{~cm}$ und $80 \mathrm{~cm}$ Entfernung vom Wurzelstock liegen.

Ein grundsätzlich anderes Verfahren wird von PUHE (1994) angewendet. Bei ihm bilden Verzweigungen und nicht Radien des Wurzelsystems die Datengrundlage. Die Wurzeldurchmesser und die Koordinaten werden an jeder Verzweigung gemessen. Dabei handelt es sich um eine sehr genaue Methode, die pro Baum mit einem BHD von $2 \mathrm{~cm}$ bis $21 \mathrm{~cm}$ einen Zeitaufwand von zwei bis acht Wochen benötigt. Bei dieser Arbeit war die Zielsetzung eine andere, so daß ein grundsätzlich anderes Konzept der Wurzelvermessung verwendet wurde.

Die Vielzahl der Aufnahmemethoden erschwert die Vergleichbarkeit der Ergebnisse. Die Anwendung eines einheitlichen Verfahrens erscheint daher empfehlenswert. Die hier gewählte Methode bietet sich dafür an, weil das Ergebnis bei hinreichender Genauigkeit durch eine hohe Stichprobengröße abgesichert werden kann. Im Falle einer Verwendung von Aufnahmeradien ist es notwendig, deren Größe bei einer BHD-Klasse gleich zu gestalten.

Tabelle 71: Radiengrößen zur Wurzelquerschnitterfassung

\begin{tabular}{|clll|}
\hline & \multicolumn{3}{l|}{ Radiengröße $(\mathrm{cm})$} \\
BHD des Baumes $(\mathrm{cm})$ & Sektion 1 & Sektion 2 & Sektion 3 \\
$<15$ & 20 & 40 & 70 \\
$16-30$ & 30 & 60 & 100 \\
$>30$ & 40 & 80 & 120 \\
\hline
\end{tabular}

\subsection{Veränderung der Wurzelarchitektur bei Mischung von Buche und Fichte}

\subsubsection{Vorbemerkungen}

Anlaß zu Mischbestandsuntersuchungen an Buche und Fichte geben u.a. Beobachtungen von FÖLSTER et al (1991) und MACKENTHUN (1990). Sie finden heraus, daß in Mischbeständen eine Verflachung der Fichtenfeinwurzeln und eine Tiefenverlagerung der Buchenfeinwurzeln stattfindet. Diese Aussagen bestätigt ROTHE (1997). Er erörtert, daß Fichten und Buchen eine direkte Wurzelkonkurrenz vermeiden. Ihre Feinwurzeln überlappen sich nur bis zu einer Tiefe von $30 \mathrm{~cm}$. Er führt weiterhin an, daß sich die Durchwurzelungsintensität bei der Fichte im Mischbestand erhöht. Dabei erschließt die Mischbestandsfichte bei halber Stammzahl den Boden fast so intensiv wie die Reinbestandsfichte. Dagegen sind bei der Buche deutlich geringere Feinwurzelmassen im Mischbestand $\mathrm{zu}$ finden als im Reinbestand. Die Ursachen für diese Ergebnisse sind noch wenig bekannt. Als mögliche Erklärung führt WITTKOPF (1995) die unterschiedliche Anfälligkeit der Baumarten gegenüber Protonentoxizität und allelopathischen Stoffwechselprodukten der Fichte an. Das Auftreten solcher Effekte wurde bei unseren heimischen Baumarten wiederholt nachgewiesen (LEIBUNDGUT 1975). Von WEISSEN und VAN PRAAG (1991) wird die Beeinflussung des Wurzelwachstums durch Huminstoffe diskutiert. Diese speziellen Auswirkungen sind jedoch im Freiland bis jetzt wenig erforscht.

Weiterhin berichten RYSAVI und ROLOFF (1994) sowie BÜTTNER und LEUSCHNER (1994) von vertikalen Verdrängungen der Buchenwurzeln durch die Mischung mit Esche oder Eiche. Standortsabhängig wird beim Vergleich verschiedener Fichtenmischbestände eine unterschiedliche Tiefendurchwurzelung festgestellt. Dabei wird auf Stagnogley eine Konzentration der 
Wurzeln im Auflagehumus (CHAPMAN et al 1986) beobachtet, während auf Moorböden Wurzeln in tieferen Bodenschichten gefunden werden (MCKAY und MALCOM 1988).

BONNEMANN (1939) untersucht die Wurzelentwicklung von einem rund 100jährigen Kiefern/Buchenmischbestand auf tiefgründigem sandigem Lehm und beobachtet die größte Wurzelmasse der Kiefern in $1 \mathrm{~cm}$ bis $20 \mathrm{~cm}$ Bodentiefe. Dagegen sind in einer Bodentiefe von 20 bis $60 \mathrm{~cm}$ erhebliche Wurzelmengen der Buchen $\mathrm{zu}$ finden. HENDRIKS und BIANCHI (1995) konnten bei Douglasien-Buchenmischbeständen eine verbesserte Tiefendurchwurzelung beider Baumarten nachweisen.

\subsubsection{Tiefendurchwurzelung}

Die Tiefendurchwurzelung (Vertikalbewurzelung) spielt für die Verankerung im Boden eine wichtige Rolle (COUTTS 1983) und ist ein wichtiger Zeiger der Stabilität. Sie hängt von den Faktoren Durchlüftung, Nährstoffversorgung, Tiefgründigkeit, Versauerung und Wasserversorgung des Bodens ab. Beim Vergleich maximaler Wurzeltiefen werden für die Fichte Tiefen von $6 \mathrm{~m}$ in tiefgründigem, lehmigem Sand (JÜTTNER 1954) und für die Buche Tiefen von $3 \mathrm{~m}$ auf reinem Sand (GANNBEN 1934) angegeben. PUHE (1994) findet bei einer einzelnen Fichte im ehemaligen Forstamt Ebergötzen Wurzeln in nahezu $3 \mathrm{~m}$ Tiefe. Damit kann die Aussage von VATER (1927) unterstützt werden, daß die Fichte nicht pauschal als Flachwurzler zu bezeichnen ist. Nach GRUBER (1992) zählt die Fichte zum Abiestyp, der sich durch eine zentral dominierende, geotrop wachsende Hauptwurzel auszeichnet. Allerdings liegt eine schwache Apikalkontolle über die Seitenwurzeln vor. Schon bei geringen Verschiebungen der optimalen Bodenbedingungen wird das Wachstum der Zentralwurzel gehemmt.

Nach BÜSGEN und MÜNCH (1927) und RACHTEJENKO (1952) ist bei der Fichte mit 30Jahren das Tiefenwachstum im wesentlichen abgeschlossen, so daß eine starke Veränderung der Tiefendurchwurzelung mit zunehmendem Alter nicht erwartet werden kann. Unterstïtzt wird diese Aussage durch die Tiefenwurzeln der 41- und 50jährigen Fichten aus Krebeck (Lehm) und Knesebeck (Sand). Sie wurzeln etwa genauso tief wie die älteren Fichten aus Holzerode.

Die für diese Arbeit untersuchten Fichten im Rein- und Mischbestand und Buchen im Reinbestand mit durchschnittlich gleichem BHD weisen Wurzeltiefen bis zu $120 \mathrm{~cm}$ auf. Deutlich flacher hingegen wurzelt die Buche des Mischbestandes. Die Werte für die tiefsten Einzelwurzeln der rund 60 jährigen Fichten und Buchen bewegen sich durchaus im Rahmen der in der Literatur angegebenen Daten. Im Schnitt wurzeln die Fichten im Mischbestand $85 \mathrm{~cm}$ tief und damit etwa 15\% tiefer als die Buche bzw. die Fichte im Reinbestand. Im Vergleich der beiden Reinbestände sind keine großen Unterschiede in bezug auf die Wurzeltiefe der Einzelwurzeln festzustellen. Durchschnittlich am flachsten wurzeln die Buchen des Mischbestandes $(60 \mathrm{~cm})$. Nach diesen Ergebnissen ist bezüglich der Tiefendurchwurzelung allein für die Fichte ein positiver Effekt durch die Mischung mit Buche zu beobachten, nicht jedoch für die Buche im Mischbestand. Über die Tiefendurchwurzelung der Bäume in Fichten - Buchenmischbeständen sind für die Grobwurzeln in der Literatur keine Angaben zu finden. Auch für die Feinwurzeln können bezüglich der maximalen Durchwurzelungstiefe keine Aussagen gemacht werden. Bedingt durch die Methode der Feinwurzelentnahme werden hierbei maximal Tiefen bis $60 \mathrm{~cm}$ erreicht. 


\subsubsection{Wurzelintensität}

\subsubsection{Durchmesserverteilung der Wurzeln}

Die Verteilung der Wurzeln auf verschiedene Durchmesserklassen ist bei den untersuchten ca. 60 jährigen Fichten im Mischbestand bei durchschnittlich gleichen Dimensionen ausgewogener als bei den Fichten im Reinbestand. Die Mischbestandsfichte besitzt einen signifikant höheren Anteil dickerer Wurzeln. Bei der Buche im Rein- und im Mischbestand unterscheiden sich die Durchmesser der Vertikal- und Horizontalwurzeln nur geringfügig und nicht signifikant. Durch die Begründung der Fichte im Mischbestand wird das Dickenwachstum der Horizontal- und besonders das der Vertikalwurzeln gefördert. Für die Buche kann kein positiver Effekt durch die Mischung mit Fichte erkannt werden.

\subsubsection{Räumliche Verteilung der Wurzeln}

Bei der relativen Verteilung der Wurzelanzahl sind bei der reinen Buche stammnah rund 15\% weniger Horizontal- und Vertikalwurzeln zu finden als bei der Mischbestandsbuche. Entsprechend mehr Wurzeln sind in entfernteren Distanzen vom Wurzelstock zu beobachten. Beim Vergleich der Relationen zwischen Horizontal- und Vertikalwurzeln ist bei den Buchen eine gleichmäßige Verteilung der Wurzeln über die verschiedenen Sektionen festzustellen. Dabei weist die Buche im Reinbestand geringfügig ausgewogenere Verhältnisse auf als die Buche im Mischbestand. Aber nicht nur die Wurzelanzahl, sondern auch die Wurzelquerschnittfläche ist bei der Durchführung eines Vergleichs zwischen den Rein- und Mischbeständen von Bedeutung. Aus diesem Grund erfolgt eine Betrachtung der relativen Abnahme der Wurzelquerschnittfläche mit zunehmender Entfernung von der Stammbasis. Die vertikale Wurzelquerschnittfläche der Buche im Reinbestand ist absolut stärker als im Mischbestand. Dennoch erfolgt die relative Abnahme der Querschnittfläche im ähnlichem Maße. Allerdings werden die Wurzeln der Mischbestandsfichte ab einer Tiefe von $40 \mathrm{~cm}$ nicht dicker. Die Buche muß demnach Faktoren ausgesetzt sein, die den Anstieg der Vertikalwurzelquerschnittfläche begrenzen. Eine Ursache dafür könnten die Fichtenwurzeln sein, die in dieser Tiefe noch große Wurzelquerschnittflächen aufweisen. Damit konkurrieren sie direkt mit den Buchenwurzeln (Abschnitt 4.1.1). Dabei können eventuell allelopathische Wirkungen eine Rolle spielen (WITTKOPF 1995).

Die Reinbestandsfichte weist horizontal nahezu die gleiche Verteilung der Wurzeln auf wie die Fichte im Mischbestand. Die meisten ihrer Wurzeln sind in einer Tiefe von $60 \mathrm{~cm}$ und bei einer Stamm(Stock-)entfernung (horizontal) von einem Meter zu finden. Die Mischbestandsfichte zeigt in der Tiefe eine höhere Wurzelintensität als die des Reinbestandes. Die meisten ihrer Wurzeln erreichen eine Tiefe von einem Meter. In der horizontalen Wurzelrichtung ist die größte Querschnittfläche der Wurzeln beider Bestände in Stammnähe zu finden. Dies läßt sich bei der Mischbestandsfichte für die Vertikalwurzeln ebenfalls feststellen. Dagegen weist die Reinbestandsfichte in $60 \mathrm{~cm}$ Tiefe die größte Querschnittfläche auf. Dabei darf nicht vergessen werden, daß die absolute Querschnittfläche der Mischbestandsfichte horizontal um $11 \%$ und vertikal dreimal so hoch ausfällt. Es zeichnet sich $a b$, daß die Reinbestandsfichte in der Tiefe ihre geringe Vertikalwurzelmenge durch einen großen Wurzelquerschnitt kompensiert. Auf diese Weise kann sie eine ausreichende Standfestigkeit (Stabilität) erreichen. Wird das Ge- 
samtwurzelvolumen betrachtet, zeigt die Fichte im Mischbestand 20\% höhere Werte als die Fichte des Reinbestandes. Dies kommt, wie oben dargestellt, durch das geringere Vertikalwurzelvolumen zustande. Der Boden wird im Mischbestand intensiver in der Tiefe durchwuzelt als im Reinbestand. Diese Resultate entsprechen nicht den Feststellungen von MACKENTHUN (1990), FÖLSTER et al (1991) und ROTHE (1997), die eine Verflachung der Fichtenwurzeln im Mischbestand beobachten. MACKENTHUN (1990) und ROTHE (1997) weisen diesen Effekt bei Feinwurzeln nach. In der vorliegenden Untersuchung stellt sich die Verteilung der Grobwurzeln anders dar als die Verteilung der Feinwurzeln. Die Grobwurzeln der Fichte im Reinbestand weisen in der Horizontalen erheblich mehr Feinwurzeln auf als die im Mischbestand, was bedeutet, daß die Durchwurzelungsintensität der Feinwurzeln im Oberboden zunimmt. Dies wäre eine mögliche Erklärung der Ergebnisse von MACKENTHUN (1990) und ROTHE (1997), die ebenfalls eine Zunahme der Feinwurzelintensität beobachten. WIEDEMANN (1943) stellt fest, daß der Unterschied zwischen Misch- und Reinbeständen meist sehr gering ist. Anhand seiner Untersuchungen wurzelt die Fichte im Mischbestand nicht tiefer als im Reinbestand, und auch die Buche zeigt im Mischbestand keine Unterschiede.

FÖLSTER et al (1991) untersuchen nicht nur Fein-, sondern auch Grobwurzeln von 60jährigen Fichten und 95- bis 160 jährigen Buchen in einzelstamm- und 60jährige Bäume in truppweiser Mischung. Bei beiden Mischungsformen stellen sie eine vertikale Wurzelraumaufteilung fest. In der Tiefe von $20-30 \mathrm{~cm}$ halten sich Fichten und Buchenwurzeln die "Waage", während darüber die Fichte und darunter die Buche dominiert. Diese im Vergleich zu der vorliegenden Arbeit konträren Ergebnisse können bei den von FöLSTER et al (1991) untersuchten Fichten und Buchen der einzelstammweisen Mischung darin begründet sein, daß ein großer Altersunterschied vorliegt. Bei den gleichalten truppweise gemischten Fichten stellt sich nach bodenkundlichen Untersuchungen heraus, daß die Fichten auf wesentlich stärker stauwasserbeeinflußtem Boden stocken als die Buchen. Diese Außenbedingungen könnten eine Begründung der gegenteiligen Ergebnisse sein.

Die verschiedenen Resultate weisen darauf hin, daß bezüglich der Wurzelausprägung in Mischbeständen noch keine eindeutigen Aussagen getroffen werden können und somit weitere Untersuchungen durchgeführt werden müssen.

Im folgenden sei kurz die Relation der Horizontal- und Vertikalwurzeln (Anzahl und Querschnittfläche) als weiterer Indikator der Wurzelintensität dargestellt. Die Ergebnisse charakterisieren die Verteilung der Wurzelbiomasse in einem definierten Bodenraum. Nach GRUBER (1992) ist dieses Verhältnis ein Maß der mechanischen und physiologischen Verankerung.

Die Buchen zeigen bei der Wurzelquerschnittfläche ein ausgewogeneres Verhältnis zwischen den Horizontal- und Vertikalwurzeln als die Fichten. Für die Wurzelanzahl kann diese Aussage nicht gemacht werden. Diese Verhältnisse charakterisieren das Senkerwurzelsystem der Fichte und das Herzwurzelsystem der Buche. Erstere zeigt wenige, im Querschnitt sehr starke Horizontalwurzeln, von denen viele dünne Senkerwurzeln in die vertikale Richtung abgehen. Das Herzwurzelsystem der Buche ist gekennzeichnet durch eine Vielzahl schwächerer Wurzeln, die sich bis in tiefe Bodenschichten verteilen und den Bodenraum sehr intensiv, netzartig erschließen. Interessant erscheint hierbei, daß die Fichte des Mischbestandes in der Verhältnisbetrachtung sich der Buche annähert. 


\subsubsection{Sproß / Wurzelbeziehungen}

Relationen ober- zu unterirdischen Parametern bei Mischbeständen (Fichte/Buche) sind in der Literatur nicht beschrieben worden. Die eigenen Untersuchungen weisen Zusammenhänge zwischen Kronen-, Stamm- und Wurzelgrößen auf, die mit zunehmendem Alter geringer werden. Ähnliche Aussagen macht MELZER (1962) in seiner Arbeit über stochastische Beziehungen verschiedener Sproß/Wurzelmerkmale von 4- und 50jährigen Reinbestandsfichten.

Das Verhältnis zwischen Stamm- und Wurzelvolumen liegt bei der Fichte im Reinbestand durchschnittlich bei 8,4:1 und bei der des Mischbestandes bei 6,9:1, wobei diese Unterschiede nicht signifikant sind. Im Hinblick auf die Einzelbaumstabilität stellt sich dieses Ergebnis für die Fichte im Mischbestand positiver dar als für die im Reinbestand. Hinzu kommt, daß die Fichte im Reinbestand erstaunlicherweise eine um $24 \%$ höhere Kronenmasse besitzt als die im Mischbestand. Dieses Resultat widerspricht Aussagen von ScHÜTZ (1989). Er führt an, daß die Kronenaubildung der Fichte in Einzelmischung mit Buche deutlich verbessert wird. Allerdings weist die hier untersuchte Fichte des Mischbestandes einen geringeren H/D-Wert auf, so daß sich ein Zusammenhang zwischen geringem H/D-Wert und größerer Wurzelmasse ergibt. Bei den Buchen können die Zusammenhänge zwischen ober- und unterirdischen Parametern nicht gefunden werden. Gründe dafür bleiben unklar. Vermutlich sind sie baumarten- und/oder altersspezifisch. ROLOFF und RÖMER (1989) beschreiben Sproß / Wurzelsysteme von zwei Meter hohen Buchen. Sie stellen gute Beziehungen zwischen den Parametern Sproßlänge/Wurzellänge, Sproßgewicht/Wurzelgewicht und Blattfläche/Wurzelgewicht heraus. Aber schon bei diesen Untersuchungen konnte eine große Heterogenität der Wurzelsysteme festgestellt werden, so daß die Autoren ausdrücklich auf die Problematik der Grobwurzelerfassung hinweisen. Ähnliche Aussagen macht PELLINEN (1986). Aus diesen Erläuterungen kann der Schluß gezogen werden, daß die gefundene Heterogenität der hier dargestellten Untersuchungen bei der Buche altersbedingt ist.

Bei den Relationen zwischen den ober- und den unterirdischen Parametern darf die Konkurrenzsituation der Bäume nicht außer acht gelassen werden. Die in dieser Arbeit vorgestellten Konkurrenzindizes zeigen, daß die Buche im Mischbestand der größten Konkurrenz ausgesetzt ist. Dies wäre ein Ansatz, um zu erklären, warum ihr Wurzelvolumen im Gegensatz zu dem der Reinbestandsbuche geringer ausfällt. Allerdings muß dabei bedacht werden, daß der verwendete HEGYI-Index nicht zwischen inter- und intraspezifischer Konkurrenz unterscheiden kann. Aus diesem Grund können Unterschiede in Abhängigkeit von der beigemischten Baumart nicht berücksichtigt werden. Unter Umständen sind nicht die Menge, die Höhe und der Durchmesser der beigemischten Fichten für den hohen Konkurrenzdruck auf die Buche verantwortlich, sondern die Tatsache, daß es sich bei den konkurrierenden Bäumen eben um Fichten handelt.

Würden die Aussagen, die FöLSTER et al (1991), MACKENTHUN (1990) und RothE (1997) gemacht haben, auch für die Grobwurzeln zutreffen, müßte die Menge der Wurzeln der Mischbestandsbuche in den unteren Bodenschichten zunehmen. Aber gerade dort zeigt die Buche des Mischbestandes ein um die Hälfte geringeres Wurzelvolumen als die reine Buche. EHWALD et al (1961) kommen zu den gleichen Ergebnissen bei Mischung der Kiefer mit Buche. Durch Beimischung von Buche wurzelt die Kiefer intensiver und nähert sich so der Wurzelausprägung von Buchenbeständen an. 
Anhand der Ergebnisse der vorliegenden Arbeit ist festzustellen, daß die Wurzelintensität der Buche im Mischbestand deutlich abnimmt. Trotzdem ist der H/D-Wert der Mischbestandsbuche kleiner als der der reinen Buche. Unter dem Aspekt, daß die dort ermittelten Konkurrenzindizes höher ausfallen, stellt dies ein bemerkenswertes Ergebnis dar.

Als Fazit dieser Untersuchungen kann gesagt werden, daß die Einmischung von Fichte in $\mathrm{Bu}-$ chenbestände die Wurzelqualität und -quantität der eingemischten Fichte deutlich verbessert. Für die Buche hat diese Einmischung jedoch nachteilige Wirkungen. Sie wurzelt flacher und besitzt weniger Vertikalwurzeln. Hierin sind Stabilitätsverluste zu erwarten.

\subsection{Kiefer und Fichte in 20, 30 und 50 jährigen Reinbeständen}

\subsubsection{Wurzelentwicklung der Kiefer auf Sand}

\subsubsection{Wurzelmorphologie der Kiefer}

Für die Kiefer sind eine begrenzte seitliche (horizontale) Ausdehnung der Wurzeln und die starke Neigung zur Ausbildung von Vertikalwurzeln typisch. Sie zeigt sich gegenüber mechanischen Widerständen im Boden sehr empfindlich, kann aber auf Veränderungen äußerer Bedingungen, wie z.B. skelettreiche Böden, gut reagieren (WIEDEMANN 1927). Auffällig ist die strenge Trennung der Horizontal- und Vertikalwurzeln, die MOIR UND BACHELARD (1969) sowie BRAEKKE UND KOZLOWSKI (1977) an verschiedenen Kiefernarten herausstellen. Durch ihre ausgeprägte Pfahlwurzel und Wurzelplastizität (MOROSOW 1928, SCAMONI 1950, WiEDEMANN 1951) ist die Kiefer in der Lage, schwierige Standorte gut zu durchwurzeln. Abhängig ist die Pfahlwurzelausbildung von der Provenienz. So findet BIBELRIETHER (1964) bei einer ostpreuBischen Herkunft eine deutlichere Pfahlwurzelentwicklung als bei einer des Rhein-Main Gebietes. LIESE (1926) hält Unterschiede der Genotypen ebenfalls für denkbar. Nach KÖSTLER et al (1968) hängt die Pfahlwurzelausbildung neben genetischen Einflüssen primär von der Bodenart und nur sekundär vom Bodentyp ab. Nach KALININ (1983) ist eine Veränderung der Wurzelmorphologie mit steigendem Alter der Kiefern nur dann erkennbar, wenn ungehindertes Wurzelwachstum stattfinden kann. Dies bedeutet, daß keine Stauschichten oder flachgründige, skelettreiche Böden dem Wurzelwachstum entgegenwirken.

\subsubsection{Tiefendurchwurzelung}

In der Literatur wird eine maximale Wurzeltiefe der Kiefer von 9,40m angegeben (LAITAKARI 1929b). Dabei weisen die verschiedenen Beschreibungen in Abhängigkeit vom Baumalter eine hohe Variation auf (STONE und KALISZ 1991). Auf tiefgründigen Böden erreichen die Wurzeln 20 jähriger Kiefern eine Tiefe von 1,50m (KÖSTLER et al 1968), die Wurzel 30-40 jähriger Kiefern eine Tiefe von 1,80-5 m (KÖSTLER et al 1968, KALININ 1983) und die Wurzeln 40jähriger Kiefern eine Tiefe von 9,40m (LAITAKARI 1929b). In Knesebeck, einem ebenfalls tiefgründigen Standort, wird für die 20jährige Kiefer eine maximale Wurzeltiefe von $0,77 \mathrm{~m}$ (Mittelwert) gefunden. Bei den 33- und 47 jährigen Kiefernbeständen wurzeln die Bäume 16\% $(0,9 \mathrm{~m})$ bzw. $56 \%(1,20 \mathrm{~m})$ tiefer. Die Unterschiede zwischen den hier festgestellten Wurzeltiefen und den entsprechenden Literaturwerten kommen dadurch zustande, daß in der Literatur "Spitzenwerte" einzelner Bäume dargestellt werden. Dagegen beziehen sich die Angaben in der 
vorliegenden Arbeit auf die Mittelwerte der maximalen Tiefe der ausgesuchten Probebäume. Die Streuung der Daten kann bis zu 30\% vom Mittelwert betragen. Darüber hinaus fehlt bei vielen Literaturangaben eine Unterscheidung zwischen Fein- und Grobwurzeln. Im Rahmen der vorliegenden Arbeit können nur Aussagen über die Tiefe der Grobwurzeln gemacht werden, da beim Umziehen der Bäume tiefer gelegene Feinwurzeln abgerissen sein dürften.

Unabhängig von diesen methodischen Problemen wird die Wurzeltiefe der Kiefer im tiefgründigen Sand von zahlreichen Faktoren beeinflußt. In dieser Beziehung ist die bereits bei der Ausprägung der Pfahlwurzel beschreibende Abhängigkeit von der Provenienz zu erwähnen. Darüber hinaus können HOCHTANNER und SEITSCHEK (1964) anhand von Meliorationsversuchen feststellen, daß die Kiefer bei einer verbesserten Nährstoffversorgung tiefer wurzelt. Ähnliches weist RASPE (1992) bei der Fichte nach.

\subsubsection{Wurzelintensität}

\section{Verteilung der stammnahen Wurzeln}

Bis zum Alter von 33 Jahren steigt in der relativen Betrachtung der Anteil der über $50 \mathrm{~mm}$ dicken Kiefernwurzeln nur wenig an (rund 15\%). Bis zum Alter von 47Jahren erfolgt eine stärkere Zunahme gerade der Vertikalwurzeldurchmesser um nahezu 50\%. In diesem Alter sind fast $20 \%$ der Vertikalwurzeln über $150 \mathrm{~mm}$ stark. Letzgenanntes deutet bei der Kiefer auf den großen Anteil der Pfahlwurzel an der gesamten vertikalen Bewurzelung hin. Ausgedrückt wird dies gleichermaßen durch die Summe der Wurzelquerschnitte, die das gesamte Wurzelsystem charakterisieren. Mit Anstieg des Alters erhöht sich der Wurzelquerschnitt sowohl horizontal als auch vertikal von 20- zu fast 50jährig auf mehr als das Zwanzigfache. Die Wurzelanzahl steigt aber nur auf das Doppelte an. Dies kann ebenfalls als Nachweis der starken Vertikalwurzeln aufgefaßt werden.

\section{Verteilung der Wurzeln mit zunehmender Entfernung vom Wurzelstock}

Die Ergebnisse der vorliegenden Arbeit zeigen, daß sich bei der Kiefer mit zunehmendem Alter die relativen Werte der Wurzelanzahl bei den verschiedenen Sektionen stark verändern. Die Kiefer legt bei Alterung neue Horizontal- und vermehrt Vertikalwurzeln an. Erstaunlich hierbei ist, daß sich die Summe der Querschnittfläche in den einzelnen Sektionen von 20- zu 47jährig in der relativen Betrachtung kaum wandelt. Dabei sind $75 \%$ der Wurzelquerschnittfläche stammnah und $25 \%$ in weiterer Entfernung zu finden. Dies deutet darauf hin, daß die Kiefer in der Jugend verstärkt Wurzeln in den tieferen Bodenschichten ausbildet und die Horizontalwurzelbildung, zeitlich gesehen, später erfolgt. $\mathrm{Zu}$ dem gleichen Ergebnis kommt WAGENHOFF (1938). Bei seinen Untersuchungen erreicht die Kiefer bereits nach drei Jahren eine Wurzeltiefe von $1 \mathrm{~m}$. Dagegen beginnen die Horizontalwurzeln erst nach 15 Jahren mit einer extensiven Ausdehnung in die Seitenbereiche. KALELA (1955) bemerkt, daß bis zu einem Alter von 40 Jahren die volle Dichte des Wurzelwerkes erreicht ist und lediglich in tieferen Bodenschichten die Anzahl der Wurzeln weiter zunimmt. Das spätere Einsetzen des horizontalen Wurzelwachstums beschreiben KÖSTLER et al (1968) bei 20-30jährigen Kiefern. Die von ihnen untersuchten Bäume weisen im hohen Alter 80\% Horizontal- und 20\% Vertikalwurzeln auf.

WAGENHOFF (1938) und KÖSTLER et al (1968) stellen weiterhin fest, daß bei der Kiefer die Querschnittfläche der Vertikalwurzeln einen Anteil von nur 20-40\% an der gesamten Wur- 
zelquerschnittfläche hat. Der entsprechende Wert liegt bei den im Rahmen dieser Arbeit durchgeführten Untersuchungen bei durchschnittlich 50\%. Wie schon KÖSTLER et al (1968) erörtern, verringert sich der Anteil der Vertikalwurzeln im Verhältnis zur Horizontalwurzelmasse im höherem Alter. Diese Abnahme kann darin begründet sein, daß die stammnahen Horizontalwurzeln sehr viel dicker werden als die Vertikalwurzeln.

\subsubsection{Sproß / Wurzelbeziehungen}

Die Ergebnisse der vorliegenden Arbeit haben gezeigt, daß sich nur bei den 20- und 33 jährigen Kiefern (nicht aber bei dem älteren Bestand) Zusammenhänge zwischen den oberund unterirdischen Baumparametern finden lassen (Abschnitt 4.3). Ebenfalls weisen KÖSTLER et al (1968) darauf hin, daß nur bei jungen Kiefern Beziehungen zwischen Baumhöhe und Wurzeltiefe zu finden sind. WIEDEMANN (1942) und BIBELRIETHER (1964) vermuten, daß schlechte Stammformen der Kiefer im Stangenholzalter mit einer flachen, einseitigen Durchwurzelung einhergehen. HILF (1927) und WAGENHOFF (1938) bemerken, daß während des ganzen Bestandeslebens kein Zusammenhang zwischen Kronen- und Horizontalwurzelausdehnung vorhanden ist. Diese Feststellungen stehen mit den geringen Korrelationen im Einklang, die zwischen den entsprechenden Parametern in dieser Arbeit ermittelt werden. Sie lassen die Schlußfolgerung $\mathrm{zu}$, daß das Wachstum der Kiefer sich mit zunehmendem Alter sehr variabel gestaltet. Beim Betrachten der Relation zwischen Stamm- und Wurzelvolumen entfallen bei jüngeren Kiefern $10 \%$ auf das Wurzelvolumen, bei den 33jährigen sind es nur 6\%. LAITAKARI (1929a) findet bei einer ca. 60jährigen Kiefer mit 0,7108 $\mathrm{m}^{3}$ Stammvolumen ein Wurzelvolumen von $0,1575 \mathrm{~m}^{3}$. Letzteres stellt einen Anteil von $16 \%$ des Wurzelvolumens am Gesamtvolumen dar. Die Größenunterschiede der Werte sind damit zu erklären, daß LAITAKARI (1929a) mit dem oben aufgeführten Wert seinen stärksten Baum angeführt hat. Bei Betrachtung der für diese Arbeit untersuchten Kiefern hat der stärkste Baum ein Stammvolumen von $0,5 \mathrm{~m}^{3}$ mit einem Wurzelvolumen von $0,08 \mathrm{~m}^{3}$. Bei diesen Werten hat das Wurzelvolumen ebenfalls einen Anteil am Gesamtvolumen von $16 \%$.

\subsubsection{Die Wurzelentwicklung der Fichte auf Sand}

\subsubsection{Tiefendurchwurzelung}

Im Schnitt erreicht eine 50jährige Fichte auf Sandboden eine Wurzeltiefe von $120 \mathrm{~cm}$. Zu ähnlichen Ergebnissen kommen HILF (1927), WITTICH (1948) und WAGENKNECHT und BELITZ (1959). Sie stellen darüber hinaus fest, daß die Fichte auf grundwasserfreien, lockeren Sanden bis $8 \mathrm{~m}$ tief wurzeln kann. Tendenziell ist zu beobachten, daß die Geschwindigkeit des Wurzelwachstums mit zunehmendem Alter abnimmt. Allerdings wird der zeitliche Ablauf dieser Entwicklung kontrovers diskutiert. Nach RACHTEJENKO (1952) soll das vertikale Wachstum der Senkerwurzeln mit spätestens 30 Jahren abgeschlossen sein. JAHN et al (1971) behaupten hingegen, daß die Tiefenwurzeln der Fichte bis zu 50Jahre später als die Haupthorizontalwurzeln angelegt werden. Dies würde bedeuten, daß die Fichte erst im höheren Alter den Unterboden erschließt. 
Bei den vorliegenden Untersuchungen läßt sich feststellen, daß die 30 jährigen Fichten im Vergleich zu den 20 jährigen Bäumen um 36\% tiefer wurzeln. Der entsprechende Wert beträgt bei den 51 jährigen Fichten $47 \%$. Dies bedeutet, daß sich das vertikale Wurzelwachstum bei fortgeschrittenem Alter der Fichten allmählich verlangsamt. Die 30 Jahre, die RACHTEJENKO (1952) als Wachstumsgrenze des vertikalen Wurzelwachstums angibt, können als ungefährer Richtwert angesehen werden. In diesem Alter verlangsamt sich das Tiefenwachstum. Es ist aber keineswegs vollständig abgeschlossen.

\subsubsection{Wurzelintensität}

\section{Räumliche Verteilung der Wurzeln}

Für die Standfestigkeit und das Wachstum der Fichten ist es entscheidend, in welcher Größenordnung der Boden durch Grobwurzeln erschlossen wird und wie sich dies mit zunehmenden Alter der Bäume verändert. Das Wurzelwachstum der Fichte zeichnet sich in den ersten Lebensjahren durch die Entwicklung der Hauptseitenwurzeln aus (HOFFMAN 1939). Diese bilden die Basis der Senkerwurzeln, die die vertikale Bewurzelung der Fichte kennzeichnen.

In dieser Arbeit wird geprüft, in welchem Verhältnis die horizontale und die vertikale Vergrößerung des Wurzeldurchmessers bei Alterung festzustellen ist. Dabei wird beobachtet, daß die horizontalen Wurzeln bei den jüngeren Bäumen größere Dimensionen erreichen als die vertikalen Wurzeln. Die junge Fichte besitzt noch keine stärkeren Vertikalwurzeln, die 50jährige weist hingegen schon über die Hälfte stärkere Wurzeln auf. Diese Beobachtung der erst horizontalen und zeitlich später vertikalen Durchmesserentwicklung kann durch die Betrachtung der Wurzelquerschnittflächen mit zunehmender Entfernung vom Stamm unterstützt werden.

Weiterhin ist mit Zunahme des Alters sowohl horizontal als auch vertikal eine Verlangsamung des Wurzelwachstums festzustellen. Der Schwerpunkt der horizontalen und vertikalen Wurzeln in ihrer Anzahl verlagert sich im Alter von 20 bis 31 Jahren in die zweite Sektion $(60 \mathrm{~cm}$ von der Stammbasis) und von 31 bis 50Jahren in die dritte Sektion $(100 \mathrm{~cm}$ von der Stammbasis). Nach KÖSTLER et al (1968) machen die Horizontalwurzeln der Fichte $60 \%$ bis sogar $100 \%$ der Gesamtwurzelmasse aus. Die Fichten des Untersuchungsgebietes in Knesebeck zeigen in der Horizontalen $80 \%$ ihrer Gesamtbewurzelung und 20\% in der Vertikalen. Mit zunehmender Entfernung vom Stamm (zweite Sektion) steigt der Anteil der vertikalen Querschnittsflächen im Verhältnis zu den horizontalen um etwa $5 \%$ an. Anzahlmäßig sind doppelt soviele Wurzeln in der Horizontalen wie in der Vertikalen zu finden. Dabei ist wiederum eine Erhöhung der Wurzelanzahl in $60 \mathrm{~cm}$ Entfernung vom Stamm erkennbar. Diese letztgenannten Merkmale charakterisieren das für die Fichte typische Senkerwurzelsystem (KÖSTLER et al 1968). Das von KÖSTLER et al (1968) beschriebene Wurzelbild kann aufgrund der in dieser Arbeit gefundenen numerischen Daten unterstützt werden. Die von ihnen dargestellte typische Vertikalwurzelbildung setzt bei einem Alter zwischen 20 und 30Jahren ein.

\subsubsection{Sproß/Wurzelbeziehungen}

Mit Zunahme des Alters ist bei den Fichten des Untersuchungsgebietes in Knesebeck ein abfallendes Verhältnis des Stamm- zum Wurzelvolumen festzustellen. Im Gegensatz zur Kiefer steigt bei der Fichte der Anteil des Stammes mit Alterszunahme an. Die junge Fichte weist ein zehnmal so hohes Stamm- als Wurzelvolumen auf, die älteste nur noch ein fünfmal so hohes. 
Wird das Kronengewicht mit in die Betrachtung einbezogen, können die ungünstigen Relationen der jüngeren Bäume relativiert werden. Sie besitzen eine um über $100 \mathrm{~kg}$ im Frischezustand leichtere Krone als die ältere Fichte. Damit ist die Relation zwischen Krone, Stamm und Wurzelvolumen für den jüngeren Baum günstiger einzuschätzen als für den älteren.

\subsubsection{Wurzelentwicklung der Fichte auf Lehm}

Das Verhältnis der Horizontal- zu den Vertikalwurzeln in ihrer Anzahl steigt bei den in Krebeck untersuchten Fichten mit zunehmendem Alter von 6:1 auf 8:1 an. Dagegen fällt die entsprechende Relation beim Betrachten der Wurzelanzahl mit zunehmenden Alter von rund 4:1 auf 3:1 ab. Demzufolge zeichnet sich der junge Bestand durch vergleichsweise viele, relativ dünne Horizontalwurzeln aus. Mit zunehmenden Alter fällt die Anzahl der Horizontalwurzel geringer aus. Dafür sind diese um so dicker. Die vertikalen Wurzeln der jungen und mittelalten Fichten haben am Gesamtvolumen einen Anteil von rund 12\%. Diese Ergebnisse sind bei Annahme der gleichen Dichte bei Fichtenwurzelholz mit den Untersuchungen von DREXHAGE (1994) vergleichbar. Er fand bei um 40jährigen Fichten (Harz) Anteile der Vertikalwurzelmasse von $4 \%$ bis $31 \%$ an der Gesamtwurzelmasse. DO-HYUNG LEE (1998) untersucht ebenfalls etwa 30 jährige Fichten. Seine Aufnahmeflächen liegen geographisch nicht weit von den in dieser Arbeit untersuchten Flächen entfernt. Er ermittelt in rund einem Meter Entfernung vom Stamm eine doppelt so große Querschnittfläche der Horizontalwurzeln und eine dreimal so große der Vertikalwurzeln. Stammnah sind die Daten von DO-HyUNG LEE (1998) in der Horizontalen mit den Daten aus Krebeck vergleichbar. In Krebeck sind demnach geringer dimensionerte Wurzeln in tieferen Bodenschichten zu finden. Dabei hat dieser Bestand eine etwa doppelt so hohe Stammzahl / ha wie der von DO-HYUNG LEE (1997) untersuchte. Weiterhin ist der Boden in Ebergötzen, ein Kolluvium, besser zu durchwurzeln als der in Krebeck. Dieser zeigt in $70 \mathrm{~cm}$ Tiefe schwerer zu durchwurzelnde, tonreichere Bodenschichten. Die Unterschiede der Wurzelquerschnittflächen könnten demnach durch die höhere Stammzahl und/oder den tonreicheren Boden in Krebeck zustande kommen. Hierin sind nach GRUBER (1992) Verluste in der mechanischen und physiologischen Stabilität zu vermuten.

\subsection{Einfluß des Bodens auf das Wurzelwachstum 30 jähriger Fichten (Sand / Lehm)}

\subsubsection{Tiefendurchwurzelung}

Die Fichten wurzeln auf Lehm schneller in die Tiefe als auf Sandboden. Allerdings erreichen ihre Wurzeln auf dem erstgenannten Standort eine geringere Tiefe. $\mathrm{Zu}$ dem gleichen Ergebnis kommt WIEDEMANN (1951). Seine Untersuchungen beziehen sich auf grundwasserferne Sandstandorte. Dagegen stellen KRAUSS $(1935,1938)$ und JÜTTNER (1954) auf trockenen ebenfalls grundwasserfernen Sandstandorten ein flaches und extensives Wurzelwachstum einzelner Fichten fest. Dieses Ergebnis kann im Rahmen der vorliegenden Untersuchungen nicht bestätigt werden. Da von KRAUSS (1935, 1938) und JÜTTNER (1954) nur wenige Bäume untersucht wurden, könnte dies der Grund für die differierenden Resultate sein. Darüber hinaus wird in letzt genannten Arbeiten keine Angabe zur Nährstoffversorgung des Bodens gemacht, die aus- 
schlaggebend sein kann für die unterschiedliche Durchwurzelungstiefe (STONE und KALISZ 1991).

\subsubsection{Wurzelintensität}

Sowohl in der Vertikalen als auch in der Horizontalen zeigen die Fichten bei gleichem Alter auf Pseudogley-Parabraunerde im Schnitt größere Wurzelquerschnittflächen als die auf dem Sandstandort. Dies läßt sich unabhängig von der Entfernung zur Stammbasis feststellen. Aber nicht nur die mittlere Querschnittfläche, sondern auch die Anzahl der Wurzeln fällt auf dem besseren Standort größer aus. Davon bilden lediglich die Tiefenwurzeln eine Ausnahme. Hier weisen die Bäume auf Sandboden in Stammnähe mehr Wurzeln auf. Darüber hinaus ist das Gesamtvolumen der Wurzeln bei den auf Sand gewachsenen Fichte geringfügig geringer.

Die dargestellten Verhältnisse belegen eine geringere Wurzelintensität der auf Sand stockenden Fichte. Allerdings sind bei diesen Bäumen in rund einem Meter Entfernung von der Stammbasis mehr Wurzeln zu finden als bei denen des besseren Standortes. Dies könnte ein Merkmal sein, daß auf eine im späteren Alter extensivere Wurzelbildung der Fichte auf Sand hindeutet. Dadurch kann der Wurzelballen vergrößert werden, und der Baum steht stabiler (NIELSEN 1990).

Gegenüber den auf Sandboden wachsenden Fichten zeigen die Bäume auf Lehm ein unausgewogeneres Verhältnis der Horizontal- zu den Vertikalwurzeln. Darüber hinaus haben die Fichten auf Sand wesentlich mehr Feinwurzeln als die auf Lehm. Dies deutet darauf hin, daß die knapperen Wasser- und Nährstoffressourcen von ihnen effektiver genutzt werden können (MARSCHNER 1986, MurACH 1989, SCHLICHTER et al 1983). Die Tatsache, daß die Fichten auf Sand tiefer, extensiver und gleichmäßiger wurzeln, eine geringere Grobwurzelmasse aber weitaus mehr Feinwurzeln besitzen, bestätigt die Ausführung von FIEDLER et al (1963). Sie stellen heraus, daß die Fichte auf schlechterem Boden eine annähernd gleiche Grobwurzelintensität zeigt, die Feinwurzelmenge jedoch stark zunimmt. Die vorliegenden Untersuchungen zeigen, daß diese Zunahme hauptsächlich in den tiefer liegenden Bodenschichten stattfindet. Da die Fichten auf grundwasserfernem Sand schlechter an Wasser und Nährstoffe gelangen, läßt sich eine Verlagerung der Wurzelmasse leicht nachvollziehen. Dieser Ansicht sind auch SCHMIDTVoGT et al (1987). Sie führen an, daß der Baum immer bestrebt ist, seine Wurzel in tiefere, feuchte Bodenbereiche zu bekommen. Dies erreicht er, indem er immer neue Wurzeln in der Tiefe ausbildet.

\subsubsection{Sproß / Wurzelbeziehungen}

Anhand der Sproß- und Kronenparameter, die in Tabelle 72 für ein beispielhaft gewähltes Wurzelvolumen von $0,028 \mathrm{~m}^{3}$ dargestellt sind, wird deutlich, daß sich die Fichte auf Sand nur geringfügig (nicht signifikant) von der Fichte auf Lehm unterscheidet. Diese errechneten Daten unter standardisierten Bedingungen stellen sich anders dar als die durchschnittlichen Daten der Probebäume. BURGER (1930), KRAUSS et al (1935) und FIEDLER et al (1963) stellen fest, daß die Fichten auf schlechtem Boden, um gleiche Wuchsleistungen zu erreichen, mehr Wurzeln benötigen als die auf gutem Boden. Demzufolge müßte die Fichte auf Lehm für die Entwicklung ihrer oberirdischen Baumkompartimente eine geringere Wurzelmasse benötigen als die Fichte auf Sand. Eine Erklärung dafür könnte bei GRACE (1997) zu finden sein. Demnach be- 
stimmt das $\mathrm{C} / \mathrm{N}-$ Verhältnis in den Blättern die Allokation der Assimilate. Bei einer ausreichender Stickstoffernährung werden vorwiegend Blätter gebildet. Dagegen kommt es bei Unterversorgung zu einer verstärkten Ausbildung von Wurzeln, um den entstehenden Mangel zu kompensieren. Dies bedeutet, daß die Bäume auf guten Standorten vorrangig ihre Blattfläche und nachrangig ihr Wurzelwerk ausbauen. Diese Ergebnisse von KRAUSS et al (1935), FIEDLER et al (1963) und GRACE (1997) stimmen nicht mit den für diese Arbeit gemachten Untersuchungen überein. Ein Grund dafür könnte sein, daß die Fichten auf Sand über einen größeren Standraum verfügen und die N-Versorgung auch auf Sand optimal ist, wie die auf Lehm. Ein Unterschied der sozialen Stellung ist für den standardisierten Baum nicht festzustellen. Die Bäume mit einem Stammvolumen von $0,28 \mathrm{~cm}^{3}$ sind auf beiden Standorten gleichsam herrschend.

Tabelle 72: Sproß - und Kronenparameter zweier Fichten mit einem beispielhaften Wurzelvolumen von $0,028 \mathrm{~m}^{3}$ auf Lehm und auf Sand

\begin{tabular}{|ccccccc|}
\hline $\begin{array}{c}\text { Wurzelvolumen } \\
0,028 \mathrm{~m}^{3}\end{array}$ & BHD $(\mathrm{cm})$ Höhe $(\mathrm{m})$ & $\mathrm{H} / \mathrm{d}$-Wert & $\begin{array}{c}\text { Stammvolu- } \\
\text { men }\left(\mathrm{m}^{3}\right)\end{array}$ & $\begin{array}{c}\text { Kronengewicht } \\
(\mathrm{kg}) \text { frisch }\end{array}$ & $\begin{array}{c}\text { Blatt }(\mathrm{kg}) \\
\text { frisch }\end{array}$ \\
Lehm & 17,3 & 15,5 & 89,0 & 0,19 & 59,7 & 31,4 \\
Sand & 16,0 & 15,3 & 96,0 & 0,17 & 64,4 & 30,5 \\
\hline Differenz \% & 8 & 1 & -8 & 11 & -8 & 3 \\
\hline
\end{tabular}

\subsection{Einfluß der Verjüngungsmethode und unterschiedlicher Bodentypen (Stagnogley, Lehm) auf die Wurzelausbildung der Eiche}

\subsubsection{Eiche auf problematischen Böden (Stagnogley)}

Aus ökonomischen Gründen wurde die Fichte aufgrund ihrer hervorragenden Wüchsigkeit im letzten Jahrhundert verstärkt angebaut. Daher wurden alte Eichenstandorte nach Abtrieb der Bäume in vielen Fällen mit der schnellwüchsigen Fichte aufgeforstet. Der Anbau von Fichte bietet beträchtliche Vorteile, aber auch Risiken, die kontrovers diskutiert werden (RÖHRIG 1990). Es bestehen Zweifel, ob durch die Fichte eine Verschlechterung der biologischen und physikalischen Eigenschaften des Bodens stattfindet (BONNEMANN 1970, GENSSLER 1959).

In dieser Zeit stehen noch keine flächendeckenden Bodenkartierungen zur Verfügung, so daß oft Unwissen über die verschiedenen Bodeneigenschaften herrscht. Die Folge ist, daß die Fichte oft nicht standortsgemäß begründet wird. Manche dieser Standorte weisen Tonschichten auf, die zum einen schlecht durchwurzelbar sind und zum anderen das Regenwasser nicht in tiefere Bodenhorizonte abfließen lassen (Stagnogley). Im Sommer verschwindet dieses Stauwasser durch direkte Verdunstung und Transpiration der Pflanze, und der Boden trocknet aus. Dadurch findet ein stetiger Wechsel zwischen Wasserüberschuß und Austrocknung statt, der diese Böden kennzeichnet. Die entscheidenden Faktoren für das Wachstum der Wurzeln in diesem Boden sind die Dichtlagerung der Bodenpartikel und der zeitweilige Sauerstoffmangel.

Durch die Stürme "Wiebke" und "Vivian" (1990) sind allein in Deutschland 62,5 Mio m³ Holz (KRONAUER 1990) geworfen worden. Diese Flächen müssen mit Baumarten aufgeforstet werden, die die Standorte optimal nutzen können. Dabei ist beispielsweise in Bayern vorgesehen, $27 \%$ der Sturmwurfflächen mit Eiche wiederzubewalden (KÖNIG et al 1995). Es müssen 
Baumarten zur Wiederbeflanzung gewählt werden, die die dichten Tonschichten durchwurzeln können. Dafür eignet sich die tiefwurzelnde Eiche. Jedoch auch hier besteht Unwissen darüber, inwieweit diese Baumart mit der Verdichtung des Bodens zurechtkommt.

Bei einem der in der vorliegenden Untersuchung gewählten Standorte sind die Eichen durch Saat und Pflanzung auf einer Windwurffläche (Stagnogley) begründet worden. Bei dem anderen Standort handelt es sich um eine Aufforstungsfläche aus ehemaliger landwirtschaftlicher Nutzung. Der Boden weist eine schwache Pseudovergleyung auf, ist aber tiefgründig durchwurzelbar.

Die Eiche kann zur Neubegründung eines Bestandes gesät oder gepflanzt werden, wobei die Saat unter ökonomischen Aspekten günstiger ist. Im Vergleich zur Pflanzung liegen die Kosten für das Säen bei rund einem Drittel (BAUMHAUER 1996).

\section{Die Pfahlwurzel}

Nach Untersuchungen von WALTER (1962) ist bei der Eiche die Bildung einer Pfahlwurzel genetisch bedingt. Im höherem Alter kann die Ausprägung der Pfahlwurzel durch eine Anpassung an skelettreiche Bodenverhältnisse überdeckt werden. Bei lockerem Bodensubstrat ist sie jedoch noch lange beobachtbar, bei verdichtetem hingegen nicht (JENIK 1957). Gegenteiliges beobachtet PFEIL (1860). Er stellt fest, daß sich die Pfahlwurzeln der Eiche auf frischem, nährstoffreichen Boden zurückbilden. KÖSTLER et al (1968) untersuchen 38 auf tiefgründigem Boden stockende Saateichen. Davon bilden 25 Bäume eine Pfahlwurzel aus. In $35 \mathrm{~cm}$ Tiefe löst sich diese in viele kleine Wurzeln auf, die sich im weiteren Verlauf horizontal ausrichten. Bis dahin verringert sich der Durchmesser der Pfahlwurzel sehr schnell. Diese Beobachtung wird von KÖSTLER et al (1968) als typisch angesehen und mit einem auf der Spitze stehenden Kegel verglichen. Bei Untersuchungen zur Morphologie der Pfahlwurzel gesäter und gepflanzter Eichen sind von BIBELRIETHER (1962), KÖSTLER et al (1968), FEICHT und GEYER (1993), SCHÜTE und TAE SU (1993) und HAUSKELLER-BulLERJAHN (1997) durchgängig die Pfahlwurzelbildung der Saat und die Herzwurzelbildung der Pflanzung beschrieben. Diese Beobachtung wird auch bei den hier untersuchten Eichen gemacht. In einer Bodentiefe von $20 \mathrm{~cm}$ hat die Pfahlwurzel der Saateiche einen Anteil von über $90 \%$ an der gesamten vertikalen Wurzelquerschnittfläche. Bei der Pflanzeiche hat die stärkste Wurzel nur einen 40 prozentigen Anteil. Diese Ergebnisse lassen sich in ähnlicher Weise auf allen untersuchten Böden feststellen. KREUTZER (1961) vermutet, daß sich die Hohlräume, die nach Verrottung der Pfahlwurzeln entstehen, günstig auf den Gas- und Wasserhaushalt des Bodens auswirken. Weiterhin wird die Erschließung des Oberbodens verbessert, indem die verbleibenden Wurzelkanäle als Raum für neue Wurzeln, Mikroorganismen, Niederschlagswasser und Regenwürmer dienen. KREUTZER (1961) nimmt an, daß der vergleichsweise kleine Hohlraum, den die Pflanzeichen beim Verrotten hinterlassen, leichter zusammensackt und schneller verschlämmt, als dies bei den Saateichen der Fall ist. Es wäre ebenfalls denkbar, daß durch die höhere Wurzelanzahl, die bei der gepflanzten Eichen gefunden wird, ein größeres Netz an Wurzelkanälen nach Verrottung der Wurzeln geschaffen wird. Durch dieses wäre eine weitreichendere, feinere Erschließung des Bodens die Folge. Wie letztere Darstellungen zeigen, besteht für diesen Bereich noch großer Forschungsbedarf. 


\subsubsection{Wurzelintensität}

Die relative Verteilung der Wurzeln mit steigender Distanz vom Stamm unterscheidet sich bei Saat und Pflanzung. Die Saateiche verlagert ihr Wurzelwachstum stärker in stammnahe, tiefere Bodenhorizonte, die gepflanzten Bäume in horizontale, weiter entfernte Bodenzonen. Dies kann unabhängig vom Standort festgestellt werden.

Beim Betrachten der Wurzelanzahl kann für die Saateiche die von KÖSTLER et al (1968) beschriebene Zersplitterung der Pfahlwurzel bestätigt werden. In einer Tiefe von $40 \mathrm{~cm}$ ist eine Zunahme der Wurzelanzahl von rund $30 \%$ zu erkennen. Bei den gepflanzten Eichen sind es $20 \%$. Dies dokumentiert, daß vermutlich durch genetische Veranlagung die Pflanzeiche in nahezu demgleichen Maße ihre Wurzelanzahl in tieferen Bodenhorizonten erhöht wie die Saateiche. Dieser Effekt ist in der Horizontalen nicht festzustellen. Auf Stagnogley ist die Erhöhung der Wurzelanzahl in tieferen Bodenschichten nicht zu beobachten. Die Begründung dafür liegt in der Festlegung der in dieser Arbeit gemessenen unterschiedlichen Schnittebene. Sie wurde in $30 \mathrm{~cm}$ Tiefe gewählt. Bei den Wurzelsystemen auf Lehm lag dieser Wert bei $20 \mathrm{~cm}$. Dies kommt gerade bei der Betrachtung der ersten Sektion stark zum Ausdruck, da die Pfahlwurzel der Eichen auf Stagnogley dort schon aufgegliedert war.

Die Aufteilung der Pfahlwurzel kann auch durch die Verhältnisbetrachtung der Horizontal- zu den Vertikalwurzeln belegt werden. Dies läßt sich sehr deutlich bei den beherrschten Saateichen nachweisen. Sie zeigen stammnah vier mal mehr Horizontal- als Vertikalwurzeln. Daraus läßt sich der Schluß ziehen, daß in dieser Sektion ein Großteil der vertikalen Bewurzelung auf eine dicke Pfahlwurzel entfällt. Mit zunehmender Entfernung verkleinert sich das Verhältnis zwischen den Horizontal- und Vertikalwurzeln. Dies muß als Folge der Aufteilung der Pfahlwurzel aufgefaßt werden.

\subsubsection{Sproß / Wurzelbeziehungen}

Das Verhältnis zwischen den oberirdischen Baumkompartimenten und der Wurzel spielt für die Stabilität und Nährstoffversorgung eine große Rolle (SCHMIDT-VOGT und GÜRTH 1977, RAVEN et al 1988, KAUSSMANN und SCHIEWER 1989, JOHNSON 1993). Daher wird es in der Praxis als Qualitätskriterium für junge Baumschulpflanzen verwendet. Ein geringes Sproß / Wurzelverhältnis gewährleistet bei richtiger Pflanztechnik ein gutes Gedeihen der Jungpflanzen (SCHMIDT-VOGT 1966, MCGEE UND LOFTIS 1993). Es existieren mehrere Definitionen, die dieses Verhältnis beschreiben. Die gängigste setzt die Sproßlänge mit dem Wurzelhalsdurchmesser in Beziehung. Außerdem kann das Verhältnis des Wurzel- zum SproßTrockengewicht oder das der Wurzeltiefe zur Sproßlänge betrachtet werden. Mit zunehmendem Alter steigt das Sproß / Wurzelverhältnis der Bäume überproportional an (BAZZAZ 1997, MULLIN 1963). Anfänglich ist ein enges Verhältnis zu beobachten, das mit zunehmenden Alter der Bäume zugunsten des Sprosses ansteigt. Abhängig ist dieser Anstieg von den standortbestimmenden Größen wie Licht, Wasser, Boden, Nährstoffe und Wärme. Diese Faktoren und die zwischen ihnen bestehenden Wechselbeziehungen führen $\mathrm{zu}$ einer sehr großen Variation der Sproß / Wurzelrelation. Dabei sehen BORCHERT (1973) und CASTLE (1978) die Wurzel und den Sproß als ein voneinander abhängendes "Balancesystem" an. Wird die Wurzel verletzt, können nicht ausreichend Nährstoffe und Wasser aufgenommen werden, so daß das Sproßwachstum behindert wird. Im umgekehrten Schluß wird bei Abbruch eines Kronenteiles die Photosynthe- 
seleistung des Baumes eingeschränkt, so daß auch das Wurzelwachstum gebremst wird. ROLOFF und RÖMER (1989) untersuchen die Beziehung zwischen Wurzel und Sproß an $2 \mathrm{~m}$ hohen Buchen und stellen eine durchschnittliche Relation von einem dreifach höheren Sproß- als Wurzelgewicht fest $\left(\mathrm{R}^{2}=0,89\right)$. Dieser Wert läßt sich auch bei den herrschenden Eichen ermitteln, die in der vorliegenden Arbeit untersucht werden. Bei den beherrschten Bäumen ist ein viermal größeres Stamm- als Wurzelvolumen zu beobachten. Trotz engerer Baumabstände zeigt die 22 jährige Saateiche auf Lehm ein stärkeres Wurzelwachstum als die Pflanzeiche. Auf Stagnogley stehen die Saateichen ebenfalls enger zusammen, haben aber im Gegensatz zu den Saateichen auf Pseutogley-Parabraunerde ein geringeres Wurzelvolumen als die Pflanzeichen.

Es kann gesagt werden, daß auf Lehm die Relation zwischen Stamm und Wurzel bei den Saateichen enger ist als bei den Pflanzeichen. Wird jedoch die Krone mit in die Betrachtung einbezogen, zeigt sich, daß dieser Schluß nicht gezogen werden darf, da die Krone der gesäten Echen sehr viel schwerer ist als die Krone der gepflanzten. Die gepflanzte Eiche zeigt ein geringeres Stammvolumen, aber auch ein geringeres Kronengewicht als die gesäte, so daß sich wieder ein engeres Verhältnis zwischen Sproß- und Wurzel einstellt. Die absoluten Größen verändern sich, nicht aber die Relation der ober- zu den unterirdischen Parametern. Es stellt sich wieder ein Gleichgewicht ein, was bei erster Betrachtung der Bestandesdaten nicht offensichtlich erwartet werden konnte.

Gründe des höheren Kronengewichtes der Saatbäume könnten darin liegen, daß durch ein größeres Wurzelvolumen eine stärkere Kronenausbildung möglich ist. Da über das Bodenwasser mehr Nährstoffe aufgenommen werden, könnte des Kronenwachstum gefördert werden. Andererseits ist der Saateiche als dienende Baumart mit 7\% die Hainbuche beigemischt. Bei der Pflanzung werden insgesamt $38 \%$ Roteiche und Linde als Mischbaumarten dazugepflanzt. Letztere mußte geköpft werden, da sie sonst in den Hauptbestand einwachsen und ihn in seinem Wachstum behindern würde. Diese Köpfung wurde ein Jahr vor der Probebaumentnahme durchgeführt, so daß die untersuchten Eichen bis kurz vor ihrer Entnahme einer starken Konkurrenz ausgesetzt waren. Demnach sind zu der Pflanzung mehr und wüchsigere Hilfsbaumarten beigemischt, die mit den Eichen stärker konkurrieren. Dies könnte ein weiterer Grund dafür sein, daß die Kronen kleiner ausgebildet sind.

Auf Stagnogley zeigt die Pflanzeiche im Vergleich der absolut ermittelten oberirdischen Daten höhere Werte als die Saateiche. Mit der Berechnung der Parameter am Beispiel einer Eiche mit standardisiertem BHD zeigt sich jedoch kein großer Unterschied zwischen Saat und Pflanzung. Das Verhältnis zwischen Stamm- und Wurzelvolumen stellt sich für die Pflanzeiche geringfügig enger dar als für die Saateiche. Dabei weisen die Bäume der Pflanzung eine schwerere und größere Krone auf als die der Saat, so daß das geringfügig günstigere Stamm / Wurzelverhältnis wieder ausgeglichen sein dürfte. Der Grund für die unterschiedliche Kronenentwicklung liegt in dem weiten Standraum, der den gepflanzten Eichen zur Verfügung steht.

Unabhängig vom Standort ist für die Eiche auf Stagnogley die Sproß/Wurzelbeziehung für Pflanzung und Saat ähnlich zu beurteilen. So haben weder Dimension noch Standort wesentlichen Einfluß auf die Beziehungen zwischen Wurzel, Krone und Stamm. Besonders der fehlende Einfluß des Bodens auf die Parameter überrascht, da die Meinung besteht, daß der Standort die Wurzelausbildung stark modifiziert (KÖSTLER et al 1968). 


\subsection{Einfluß der sozialen Stellung auf die ober- und unterirdischen Baumkom- partimente}

Die soziale Stellung eines Baumes ist ein Ergebnis von kleinstandörtlichen Bedingungen wie Strahlung, Wasser (DHOTE et al 1994), Temperatur, Nährstoffversorgung (COLIN-BELGRAND et al 1993), genetisches Potential und Konkurrenzsituation (KENNEL 1966). Um Ziele der Bestandespflege charakterisieren zu können, ist es notwendig, eine Gliederung des Bestandes vorzunehmen (RÖHRIG und GUSSONE 1990). Dafür stellt die soziale Stellung im Bestand ein Kriterium auf Einzelbaumebene dar. Durch sie wird die Vitalität des Baumes bestimmt, die durch Stamm- und Kronenausbildung charakterisiert ist. Gleichaltrige Bestände können so reproduzierbar klassifiziert werden. Dabei stellt die Kraft'sche Klasseneinteilung die verbreitetste Methode dar. Allerdings weist sie auch unbestreitbare Mängel auf (ROZSNYAY 1979). Der größte Nachteil ist die subjektive Klassifizierung durch den Beobachter. KENNEL (1966) überprüft die okular bestimmten Baumklassen durch Diskriminanzanalysen. Die Übereinstimmungen der geschätzten mit den berechneten Größen betragen bei der Fichte $80 \%$ und bei der Buche $73 \%$.

Bei den meisten Bäumen ist die Abhängigkeit der Wurzelausbildung von der Ausformung der Baumkrone und damit von der sozialen Stellung des Baumes sehr goß (SCHMIDT-VoGT 1977). Ungeklärt ist die Frage, ob die Stellung im Bestand die Wurzelausbildung oder umgekehrt die Wurzelausbildung die soziale Stellung beeinflußt (WAGENKNECHT und BELITZ 1959). KÖSTLER et al (1968) vermuten in der Wurzelmorphologie sogar die Begründung des "Umsetzens" von Eliteanwärtern (herrschende Bäume) und echter Elite (vorherrschende Bäume).

Alle Bäume der vorliegenden Untersuchungen werden differenziert nach Kraft'schen Klassen ausgewählt. Dadurch läßt sich die Veränderung des Wurzelvolumens bei Rückgang der sozialen Stellung beurteilen. Dabei ist das Wurzelvolumen der vorherrschenden Bäumen und herrschenden Bäume signifikant höher als das der mitherrschenden und beherrschten. Ähnliches ist für den BHD, die Höhe, das Kronengewicht und das Stammvolumen zu beobachten. Dabei fallen BHD und Höhe durchschnittlich von Klasse zu Klasse um 20\% ab und Kronengewicht, Stamm- und Wurzelvolumen um rund 50\%. Stamm-, Wurzelvolumen und Kronengewicht gehen demnach im Gegensatz zu BHD und Höhe überproportional zurück. Die Relation zwischen Sproß und Wurzel verändert sich aber dabei nicht signifikant.

Die folgenden Überlegungen beschäftigen sich mit der Frage: "Beeinflusst die Wurzelmorphologie das Baumwachstum oder das Baumwachstum die Wurzelmorphologie?".

Dieses wird am Beispiel 22jähriger Eichen erörtert. Bei herrschenden Eichen auf Lehm ist ein signifikant größerer Anteil Horizontal- als Vertikalwurzeln zu finden, bei den beherrschten kehrt sich dieses Verhältnis um. Diese Beobachtungen sind in der Literatur in der Form noch nicht dargestellt. LEMKE (1956) beobachtet an Roteiche Quercus rubra L.), daß beherrschte Bäume im höheren Alter (67Jahre) noch reduzierte Pfahlwurzeln besitzen. Bei den Herrschenden ist dies nicht mehr zu erkennen. Sie zeigen ein ausgeprägtes Herzwurzelsystem. Obwohl es sich um eine andere Eichenart handelt, wäre dieser Wachstumsverlauf auch für die hier untersuchten Eichen denkbar, da sich die Pfahlwurzel der beherrschten Bäume sehr ausgeprägt darstellt. Demgegenüber bemerkt LEMKE (1956) jedoch, daß bei der beherrschten Roteiche (Quercus rubra L.), trotz ausgebildeter Pfahlwurzel, ein Flachwurzelsystem zu beobachten ist. Diese 
Annahme verwundert und kann anhand der für diese Arbeit gemachten Untersuchungen nicht bestätigt werden.

Um die Differenzierung der Bäume im Bestand zu erklären, wäre zuerst eine Kronenkonkurrenz denkbar. Anhand des HEGYI-Indexes ist zu erkennen, daß die Eichen der Kraft'schen Klasse 2 einer signifikant geringeren Konkurrenz ausgesetzt sind als die der Kraft'schen Klasse 4. Außerdem sind bei Letzteren die Abstände zu den benachbarten Bäumen geringer. Bei Beschattungsversuchen an jungen Waldpflanzen spielt das Licht in bezug auf das Wurzelwachstum eine große Rolle. Die Substanzproduktion der Wurzel reduziert sich bei Beschattung deutlich stärker als die der oberirdischen Pflanzenteile (RÖHRIG 1966). Diese Resultate sind für die Buche von BURSCHEL et al $(1964,1965)$ sowie HARLEY und WAID (1955), für die Traubeneiche von Brown (1955), JARVIS (1964), Ovington und MCRAE (1960) sowie RöHRIG (1958) und für die Kiefer, Eiche und Buche von LYR (1994) gemacht worden. HAUSKELLERBULLERJAHN (1997) stellt hingegen fest, daß bei Zunahme der Beschattung keine signifikanten Veränderungen der Sproß / Wurzelbeziehung zu finden sind.

Die Bäume, die in den ersten zehn Jahren die stärkste Krone ausbilden, besitzen aufgrund eines höheren Lichtgenusses eine höhere Photosytheserate und können mehr Wurzelmasse entwickeln. Diese ist zum großen Teil in den oberen Bodenschichten (horizontalen Ebene) zu finden, wo die meisten Nährstoffe vorhanden sind. Das horizontale Wurzelwachstum kann für die herrschenden Eichen beider Standorte gleichsam beobachtet werden. Mit Anlage dieser Wurzeln wäre der horizontale Bodenbereich ausgefüllt, und die in der Initialphase starken Bäume dominieren über die schwächeren Eichen durch die horizontale Ausbreitung ihrer Wurzeln. Die schwächeren Bäume verlagern ihre Wurzeln in tiefere Schichten. Dennoch können sie die Defizite nicht mehr aufholen und bleiben so in ihrem Wurzelwachstum immer stärker zurück. Auf der anderen Seite wäre es denkbar, daß es aufgrund genetischer oder kleinstandörtlicher Unterschiede zu einer stärkeren Horizontalwurzelbildung kommt. Nach FABRICIUS (1929) bewirkt der Wurzelwettbewerb bei gleichem Lichtgenuß ein bedeutendes Zurïckbleiben der Entwicklung. Auch SHAW (1974) bemerkt, daß die Wurzelkonkurrenz benachbarter Bäume und der Begleitvegetation einen größeren Einfluß auf das Wachstum junger Eichen hat als die Strahlungsintensität. Nachdem eine durch die Wurzel hervorgerufene Differenzierung im Bestand stattgefunden hat, besitzt die herrschende Eiche eine größere Wurzelmasse. Dadurch ist von einer besseren Wasser- und Nährstoffversorgung auszugehen, so daß das Sproßwachstum ebenfalls gefördert wird. Bei diesem Ansatz wäre zuerst die Wurzelkonkurrenz und nachfolgend die Kronenkonkurrenz vorhanden, während bei der ersten Überlegung erst die Kronen- und dann die Wurzelkonkurrenz einsetzten würde.

Beide Ansätze gehen von der Annahme aus, daß sich zwischen der ober- und unterirdischen Masse immer ein Balance einstellt (BORCHERT 1973, CASTLE 1978). Dabei sind "Balancespannweiten" möglich, welche durch die verschiedenen sozialen Stellungen zustande kommen.

Eine andere Möglichkeit zur Klärung der vermehrten Wurzelbildung könnte in der Windexposition der herrschenden Bäume gefunden werden. Auch wenn Eichen in einem recht engem Verband erwachsen, läßt sich eine deutliche Höhenstrukturierung des Jungwuchses erkennen. Bei den 13jährigen Bäumen in Kattenbühl ist eine Höhendifferenz der zweiten zur vierten Kraft'schen Klasse von ca. $40 \%$ zu beobachten. Dominante Bäume ragen demnach aus dem 
Bestand heraus und sind besonders an der Kronenspitze dem Wind ausgesetzt. Um ihre Stabilität zu erhöhen, bilden die Eichen verstärkt Wurzeln aus (FRITZSCHE 1933; NIELSEN 1990). Diese sind unabhängig vom Boden in horizontalen Schichten zu finden.

Es ist ebenfalls möglich, daß alle drei diskutierten Möglichkeiten zusammen das Wachstum der Eichen bestimmen. Das heißt, die Eichen wurzeln nach Keimung erst einmal in die Tiefe, wobei gleichzeitig das Sproßwachstum und eine Höhendifferenzierung einsetzen. Darüber hinaus finden einige Bäume kleinstandörtlich bessere Bedingungen vor und können so oberirdische Pflanzenteile besser versorgen. Durch die daraus folgende höhere Photosyntheserate sind diese Bäume in der Lage, verstärkt horizontale Wurzeln auszubilden. Die Horizontalwurzeln gewährleisten wiederum einen bessere Wasser- und Nährstoffversorgung, und obendrein besetzen sie den Wurzelraum. Gleichzeitig ist das Sproßwachstum gefördert, so daß die Bäume aus dem Bestand hervorragen. Somit sind sie einer verstärkten Windexposition ausgesetzt, auf die sie zur Stabilitätserhöhung mit noch stärkerem Wurzelwachstum reagieren müssen.

Inwieweit ein oder alle drei Ansätze der Realität entsprechen, kann aufgrund der statischen Aufnahmetechnik dieser Arbeit nicht beantwortet werden. Nach SZAPPANOS (1969) hängt das Wachstum des Jungwuchses bei gleichen Beleuchtungsverhältnissen von den Niederschlagsverhältnissen und dem Wasserhaushalt des Standortes ab. Er stellt bei TraubeneichenHainbuchenbeständen mit und ohne Wurzelkonkurrenz fest, daß die Nährstoffversorgung, aber besonders die Wasserversorgung ohne Wurzelkonkurrenz verbessert wird.

Nach Untersuchungen von SCHÜTE und KIM TAE SU (1993) an im Mittel 10jährigen Direktsaateichen treten neben der Pfahlwurzel keine seitlichen Hauptwurzeln auf. HAUSKELLERBULLERJAHN (1997) kommt an 6jährigen Saat- und Pflanzeichen zu gleichem Ergebnis. Bis zum Alter von 10Jahren erfolgt das Wurzelwachstum demnach überwiegend geotrop. Die Entwicklung der Horizontalwurzeln müßte so, nach den für die vorliegende Arbeit festgestellten Ergebnissen, auf Lehm zwischen 10 und 22 jährig einsetzen. Bei den 13jährigen dominanten Eichen auf Stagnogley ist eine große Horizontalwurzelmasse vorhanden. Diese dürfte nach den Ergebnissen von SchÜTE und KIM TAE Su (1993) und HAUSKELlER-BulleRJAHN (1997) zwischen 10 und 13 Jahren stattgefunden haben, was wenig plausibel erscheint. Unterschiede dürften durch die unterschiedlichen Standortsverhältnisse zustande kommen.

\subsection{Waldbauliche Schlußfolgerungen}

Es war zu klären, inwiefern sich die Grobwurzelarchitektur im Buchen-Fichten-Mischbestand gegenüber der in den Reinbeständen verändert. Die Ergebnisse, die anhand von 40 untersuchten Bäumen gewonnen werden, lassen erste Tendenzen erkennen, die in die Praxis einbezogen werden können. Die Wurzeln der etwa 60jährigen Fichte zeigen im Mischbestand deutlich bessere Merkmale als die der Fichte im Reinbestand. So konnte beispielhaft festgestellt werden, daß die Summe der Querschnittflächen der Vertikalwurzeln bei der Fichte im Mischbestand in $90 \mathrm{~cm}$ Tiefe fast fünfmal höher ist als bei der Fichte im Reinbestand. In der horizontalen Richtung besaßen die Wurzeln der Bäume beider Bestandesformen ähnliche Dimensionen. Darüber hinaus konnte für die Fichte im Reinbestand ein ungünstigeres Verhältnis des Stamm$\mathrm{zu}$ Wurzelvolumen festgestellt werden als bei der im Mischbestand. Für die Buche konnten diesbezüglich keine eindeutigen Schlußfolgerungen getroffen werden. Ansatzweise kann aus 
den Ergebnissen gefolgert werden, daß die Buche des Mischbestandes flacher wurzelt als die des Reinbestandes. Dies zeigte sich insbesondere an den vertikal verlaufenden Wurzeln, die in ihrem Querschnitt deutlich denen der Reinbestandsbuchen unterlegen waren. Es können jedoch keine signifikanten Schlüsse aus den Beziehungen zwischen ober- und unterirdischen Größen gezogen werden $\left(\mathrm{R}^{2}<0,5\right)$.

Es kann demnach empfohlen werden, die Fichte aufgrund ihres stärkeren Wurzelwachstums im Mischbestand mit Buche zu begründen. Inwiefern sich die Mischung auf die Buche auswirkt, kann nicht eindeutig festgestellt werden.

Ein weiterer Schwerpunkt dieser Arbeit liegt in der Beurteilung des Verjüngungsverfahrens der Eiche. Nach den oben diskutierten Ergebnissen sind keine eindeutigen Vorteile der Saat oder Nachteile der Pflanzung festzustellen. Erste, nach der Datenaufnahme festgestellte bessere Ertragsleistung der Saateiche auf Lehm steht die höhere Wuchsleistung der Pflanzeiche auf Stagnogley gegenüber. Nicht beurteilt werden kann in dieser Arbeit der Einfluß der Güte des Saat- und des Pflanzgutes auf die Morphologie der Wurzeln. Neben der Qualität des Saatgutes, die ausgedrückt sein kann durch die Größe der Eichel (RICE et al 1993) spielen beim Keimen vorhandene Nährstoff- und Wasserversorgung (LONG et al 1996) ebenso wie die $\mathrm{CO}_{2}$ - Konzentration (MIAO-SHILI und JONAS1996), Wärme und Licht (KOZLOWSKI 1972, MAYER UND POLJAKOFF-MAYBER 1975) eine große Rolle. Letztgenannte ökologische Kriterien werden bestimmt durch die Saattechnik (RÖHRIG 1976) und den Saattermin.

Bei der Pflanzung bestehen ebenfalls viele Kriterien, die das Wachstum des Baumes beeinflussen. Die Güte des Pflanzenmaterials hängt von Baumschulbehandlung, Aufzucht, Wurzelschnitt, Lagerung und Transport, Pflanzensortiment und letztendlich von dem Pflanzvorgang im Wald ab. Aus ökonomischer Sicht ist die Saat vorzuziehen, da sie finanziell günstiger ist (BAumhauer 1996). Einen Vorteil der Saat könnte nach KREUTZER (1961) die BodenerschlieBung durch die Pfahlwurzel darstellen. Diesem ist besonders auf Stagnogley eine große Bedeutung zuzumessen. Durch ihren großen Wurzelquerschnitt werden nach dem Absterben des Baumes vertikale Hohlräume geschaffen. Dadurch kann das Bodenwasser besser abfließen und eine ausgeprägtere Bioturbation stattfinden. Diese großen Hohlräume kann allein die Pfahlwurzel der Saat schaffen, da die Pflanzung meist keine Pfahlwurzel mehr besitzt. Inwiefern wirklich diese von KREUTZER (1961) geforderten großen Hohlräume benötigt werden, ist aus der Literatur nicht zu ersehen. Die Aussage von KREUTZER (1961) muß als Hypothese aufgefaßt werden, da keine direkten Untersuchungen zu dieser Frage dargestellt werden. Zu bedenken ist dabei, daß die gepflanzten Eichen durch viele kleine Wurzelkanäle ebenfalls Hohlräume schaffen, deren Effizienz im Vegleich zur Pfahlwurzel untersucht werden muß. Nach den vorliegenden Untersuchungen der Eiche auf Stagnogley zeigt der gesäte Baum, bei etwa gleichem Wurzelquerschnitt wie der gepflanzte, eine geringere Wurzelanzahl. Weitere Kriterien wie die relativen Betrachtungen zwischen Sproß-, Wurzelvolumen und Kronengewicht ergeben, genauso wie das Verhältnis von Horizontal- zu Vertikalbewurzelung, ebenfalls keinen Anlaß, eines der Begründungsverfahren vorzuziehen. 


\section{Zusammenfassung}

Die vorliegenden Untersuchungen sind Teil eines gemeinsamen Projektes der Europäischen Union. Es wurden dabei verschiedene Fragestellungen zu "Funktionalen Aspekten der Wurzelarchitektur und der Biomasseverteilung von sechs europäischen Hauptbaumarten" bearbeitet. Das Waldbauinstitut der Universität Göttingen untersuchte hierbei Wurzeln der Fichte, Buche, Kiefer und Eiche.

Ziel dieser Arbeit war es, zu klären, inwiefern verschiedene Faktoren wie Baumartenmischung, Alter der Bäume, Standort und Verjüngungsmethode die Grobwurzelarchitektur der Baumarten Fichte, Buche, Kiefer und Eiche beeinflussen. Dazu wurden 17 Flächen ausgewählt, in denen einzelne Fragestellungen bearbeitet wurden. Die Untersuchungsbestände liegen in den niedersächsischen Forstämtern Bovenden (Holzerode und Krebeck), Knesebeck, Holzminden und Kattenbühl.

Aus jedem Untersuchungsbestand wurden 6-16 Probebäume entnommen. Zur Klärung der Frage, wie stark ein Baum von seinen Nachbarn bedrängt war, wurden die Durchmesser der drei nächsten Nachbarbäume und die Entfernung Probebaum/Nachbarbaum ermittelt. Bei einzelnen Beständen wurden darüber hinaus die Höhe und die Kronenschirmfläche der Nachbarbäume aufgenommen. Mit diesen Daten wurden die konkurrenzbeschreibenden Indizes HEGYI-INDEX und ÜBERRAGUNGSWINKEL berechnet. Nach der Entnahme der Probebäume wurden Kronen-, Stamm- und Wurzelparameter untersucht. Das Wurzelsystem wurde zur Vermessung in Horizontal- und Vertikalebenen unterteilt. In diesen Ebenen wurden in drei unterschiedlichen Entfernungen vom Wurzelstock Winkel und Durchmesser (bis $2 \mathrm{~mm}$ ) der Wurzel gemessen. Eine Wurzel mit dem Winkel kleiner $45^{\circ}$ wurde als Horizontalwurzel, eine größer/gleich $45^{\circ}$ als Vertikalwurzel bezeichnet. Mit diesen Werten wurden Wurzeldurchmesserverteilungen erstellt, die als Grundlage für die Berechnung der Wurzelquerschnittflächen in den drei horizontalen und vertikalen Entfernungen von der Stammbasis (Sektionen) dienten. Daneben wurde für jede Einzelwurzel die maximale Tiefe ermittelt. Damit wurden die durchschnittliche und die maximale Tiefe für jedes Wurzelsystem berechnet. Weiterhin wurde die Anzahl der Feinwurzeln geschätzt, die sich an einer Einzelwurzel befanden.

Die Summe der Wurzelquerschnittflächen in Verbindung mit der Wurzelanzahl ergab einen Wert, der das Wurzelsystem charakterisierte. Anhand dieser Parameter wurden die "Relation zwischen Horizontal- und Vertikalwurzel", das "Wurzelvolumen" und die "Wurzeloberfläche" berechnet. Das Wurzelvolumen wurde, differenziert nach Horizontal- und Vertikalwurzeln, für jeden Probebaum kalkuliert. Grundlage dieser Berechnungen bildete die Querschnittflächensumme eines Wurzelsystems, die für die drei Sektionen verfügbar war. Die Beziehung zwischen Sproß und Wurzel wurde durch eine lineare Ausgleichsfunktion zwischen Stammund Wurzelvolumen beschrieben (Abb. 33). Dabei zeigten sich keine signifikanten Unterschiede zwischen den verschiedenen Bestandesformen. Zur genaueren Erfassung des bestandesspezifischen Verlaufes des Wurzelvolumens in Abhängigkeit vom Stammvolumen wurden darüber hinaus Regressionen für die einzelnen Bestände erstellt. Auch hier wurde geprüft, inwiefern sich die Regressionsgeraden statistisch voneinander unterscheiden. 
Zur detaillierten Erfassung der Sproß/Wurzelbeziehungen wurden sogenannte "Flußdiagramme" für nahezu alle Bestände erstellt (Anhang V). In diesen sechs Diagrammen wurden Regressionen zwischen Stamm- und Wurzelvolumen und den Parametern BHD, Höhe, Kronen-, Blatt- und Wurzelgewicht (nur für den Bestand Kattenbühl) gegenübergestellt. Dabei ergaben sich in den meisten Fällen gute Zusammenhänge mit durchschnittlichen Bestimmtheitsmaßen von 0,79 .

Bei allen untersuchten Beständen wurden Bäume verschiedener sozialer Stellungen entnommen. Ober- und unterirdische Baumparameter zeigten mit Abfall der Kraft'schen Klasse die erwarteten abnehmenden Werte. Dabei gingen Kronengewicht, Wurzel- und Stammvolumen deutlich stärker zurück als BHD und Höhe. Weiterhin war ein signifikanter Unterschied zwischen dem Wurzelvolumen der Bäume der Kraft'schen Klassen 1 und 2 zu den Klassen 3 und 4 zu beobachten. Auch zeigten herrschende Bäume ein schwach engeres Verhältnis zwischen Sproß und Wurzel als beherrschte. Ungeklärt ist die Frage, ob die Wurzel das Sproßwachstum oder umgekehrt das Sproßwachstum die Wurzel beeinflußt. Ob die soziale Differenzierung durch Wurzel- oder Kronenkonkurrenz zustande kommt, konnte in dieser Arbeit nicht geklärt werden. Dies wäre eine wichtige Frage, für die weitere Untersuchungen notwendig erscheinen.

Anhand von Fichten und Buchen im Rein- und Mischbestand auf Lehm wurde untersucht, welchen Einfluß die Mischung dieser Baumarten auf deren Wurzelarchitektur hat (Holzerode).

Danach zeichnet sich ab, daß die Fichte in Mischung mit Buche ein signifikant tiefer reichendes und im Querschnitt größeres Wurzelsystem entwickelt als die Fichte im Reinbestand. Die Summe der Wurzelquerschnittfläche der ersten Sektion $(30 \mathrm{~cm}$ Radius bzw. $40 \mathrm{~cm}$ Tiefe) ist bei der Fichte im Mischbestand in der horizontalen- $11 \%$ und in der vertikalen Wurzelrichtung viermal so hoch als die der Fichte im Reinbestand (Abschnitt 4.2.2.2). Die in Mischung mit Buche festgestellte Veränderung der Wurzelarchitektur betrifft demnach hauptsächlich die Tiefendurchwurzelung. Mit zunehmender Tiefe (zweite Sektion, $60 \mathrm{~cm}$ ) besitzt die Fichte im Mischbestand noch eine $40 \%$ größere Wurzelquerschnittflächensumme als im Reinbestand, in der dritten Sektion (90 cm Tiefe) beträgt dieser Wert $80 \%$. Demnach ist das Verhältnis zwischen Horizontal- und Vertikalbewurzelung bei den Fichten im Mischbestand enger als bei den Fichten im Reinbestand. Diese Ergebnisse kennzeichnen das in Richtung Herzwurzelsystem tendierende Wurzelsystem der Mischbestandsfichte und das Senkerwurzelsystem der Fichte im Reinbestand. Weiterhin ist bei den Fichten des Mischbestandes eine signifikant geringere Anzahl an Feinwurzeln festzustellen als bei den Reinbestandsfichten.

Die Buche im Mischbestand zeigt ein flacheres Wurzelsystem mit einer geringeren Wurzelquerschnittfläche als die Buche im Reinbestand. In der horizontalen Wurzelrichtung besaß sie eine $10 \%$ kleinere Wurzelquerschnittfläche (nicht signifikant). In der Tiefe war jedoch die Wurzelquerschnittfläche (signifikant) nur halb so groß. Im Vergleich der vier untersuchten Bestände war die Buche im Reinbestand allerdings durch ihre Nachbarbäume am stärksten bedrängt. Dies zeigten die errechneten Konkurrenzindizes. Demnach kann das Ergebnis der flacher und schwächer wurzelnden Buchen des Mischbestandes vermutlich nicht auf Bestände mit konkurrenzstärkerer Buche übertragen werden. Die Resultate dieser Untersuchungen zur Grobwurzelarchitektur von Mischbestandsbäumen sind in der Literatur nicht beschrieben und 
erfordern weitere Forschung. Sie lassen die Schlußfolgerung zu, daß die Begründung von Mischbeständen mit Fichte und Buche durchaus Vorteile für das Wurzelsystem der Fichte hat.

Einen nächsten Schwerpunkt bildeten 21-, 30- und 41 jährige Fichten auf ähnlichem Standort (Lehm). Anhand dieser drei Bestände sollte geklärt werden, wie sich das Wurzelsystem und oberirdische Größen mit zunehmendem Alter verändern. Dabei wird deutlich, daß die Tiefendurchwurzelung mit Anstieg des Alters kontinuierlich zunimmt. Dies stellt ein interessantes Ergebnis dar, da nach RACHTEJENKO (1952) das vertikale Wachstum der Senkerwurzeln mit 30 Jahren nahezu abgeschlossen sein soll, in Krebeck jedoch bis zum Alter von 41 Jahren noch eine Zunahme der Tiefenerschließung des Bodens beobachtet wurde. Allerdings verlangsamt sich dieser Vorgang. Während die Summe der Wurzelquerschnittfläche in $40 \mathrm{~cm}$ Tiefe vom Alter 21 bis 30 Jahre auf das Dreifache zunahm, stieg sie von 31 bis 40 Jahre nur noch um die Hälfte an.

Die Frage, wie sich ober- und unterirdische Größen mit zunehmendem Alter ändern, wurde auch an Fichte und Kiefer auf Sandboden (Knesebeck) bearbeitet. Hierfür wurden drei 20 bis 50 jährige Bestände jeder Baumart ausgewählt. Zusätzlich konnte an zwei 30 jährigen Fichtenbeständen analysiert werden, inwiefern standörtliche Unterschiede das Wurzelwachstum (auf Sand und auf Lehm) verändern. Die Fichte auf Sand wurzelte durchschnittlich tiefer als die auf Lehm. Auch in Knesebeck konnte bei der Fichte beobachtet werden, daß sich die Geschwindigkeit der Tiefendurchwurzelung im höheren Alter verlangsamt. Dabei steigt die Summe der Wurzelquerschnittfläche in $40 \mathrm{~cm}$ Tiefe von 20 bis 31 Jahren auf das Achtfache. Bei Alterszunahme um weitere zwanzig Jahre erhöht sich dieser Wert aber auf nur knapp das Dreifache. Bei den ober- und unterirdischen Größen ist kein signifikanter Unterschied zwischen den Fichten auf Lehm und denen auf Sand zu erkennen(S.90).

Für die Kiefer konnte ebenfalls ein kontinuierlicher Anstieg der Durchwurzelungstiefe mit alnehmendem Alter festgestellt werden. Die Summe der Wurzelquerschnittfläche stieg vom Alter 20 bis 33 Jahre in der vertikalen Richtung auf mehr als das Vierfache an und in der Horizontalen auf das Zwölffache. Die Relation zwischen den Horizontal- und den Vertikalwurzeln war bei der Kiefer sehr viel enger als bei der Fichte. Darin drückt sich das für die Kiefer typische Pfahlwurzelsystem aus. Das Verhältnis zwischen ober- und unterirdischen Größen wies für ältere Kiefern eine große Streuung auf.

Untersuchungen an 13- bzw. 22 jährigen Eichen auf zwei verschiedenen Standorten (Holzminden: Lehm; Kattenbühl: Stagnogley) sollten Unterschiede der Wurzelarchitektur herausstellen, die aus verschiedenen Verjüngungsverfahren (Pflanzung und Saat) resultieren. Die Saateiche auf Lehm wies höhere ober- und unterirdischen Wuchsleistungen auf als die gepflanzte Eiche. Auf Stagnogley besaß die Saateiche eine geringere Wuchsleistung als die gepflanzten Eiche. Die Wurzeln der Saateiche zeigten auf Lehm in der vertikalen Wurzelrichtung durchschnittlich weniger Vertikalwurzeln. Sie besaßen aber in $20 \mathrm{~cm}$ Tiefe eine doppelt so große Wurzelquerschnittflächensumme wie die gepflanzten Eichen. Diese Unterschiede hoben sich jedoch in tieferen Bodenschichten wieder auf. Bei den Bäumen auf Stagnogley zeigte die gepflanzte Eiche in der Horizontalen eine größere Wurzelquerschnittfläche als die gesäte Eiche. In der vertikalen Wurzelrichtung waren nur geringfügige, nicht signifikante Unterschiede festzustellen. Durch Berechnungen unter standardisierten Bedingungen konnte herausgestellt werden, daß 
die Beziehung zwischen ober- und unterirdischen Größen sehr ähnlich zu bewerten ist. Für die Eichen beider Standorte wurde beobachtet, daß bei Saateichen die Pfahlwurzel an der gesamten vertikalen Bewurzelung einen größeren Anteil hatte als bei Pflanzeichen. Die Pflanzeichen wiesen viele, im Querschnitt kleinere vertikale Wurzeln auf und erreichten somit ähnliche Wurzelquerschnittwerte. Der Unterschied der Tiefendurchwurzelung zwischen gesäten und gepflanzten Eichen war nicht signifikant. Im Vergleich beider Standorte wurzelten die Eichen auf Stagnogley durchschnittlich erstaunlicherweise fast genauso tief wie auf Lehm, obwohl sie jünger waren.

Insgesamt konnten in der Tiefendurchwurzelung und der Wurzelquerschnittfläche keine signifikanten Unterschiede zwischen gepflanzten und gesäten Eichen gefunden werden. Da andere Untersuchungen gegensätzliche Resultate erbrachten, besteht auch hier Forschungsbedarf. Darüber hinaus konnte keine eindeutige Aussage zur Hemmung des Wurzelwachstums der Eiche auf Stagnogley gemacht werden. Hierfür wären Wiederholungen in späteren Jahren sehr wünschenswert. 


\section{Glossar}

Buche

Buche / Fichte (Bu / Fi)

Einzelwurzel

Feinwurzeln (KÖSTLER et al Feinstwurzeln $<1 \mathrm{~mm}$ 1968)

Feinwurzeln im engeren Sinne 1-2mm

Schwachwurzeln 2-5mm

Fichte

Fichte / Buche (Fi / Bu) waren waren
Probebäume sind Buchen, die von bis zu 10 Buchen umgeben

Probebäume sind Buchen, die von bis zu 10 Fichten umgeben waren

Eine Wurzel des gesamten Wurzelsystemes

Probebäume sind Fichten, die von bis zu 10 Fichten umgeben

Probebäume sind Fichten, die von bis zu 10 Buchen umgeben waren

$\mathrm{GHA}=$ Gesamte horizontale Summe aller horizontalen Wurzeln in ihrer Anzahl, unter $45^{\circ}$ Wurzelanzahl in die Tiefe gehende Wurzeln der drei Sektionen

GHQ = Gesamte horizontale Summe aller horizontalen Wurzelquerschnittflächen, unter Wurzelquerschnittfläche $45^{\circ}$ in die Tiefe gehende Wurzeln

GPA Summe aller unter $60^{\circ}$ in die Tiefe gehenden Pfahlwurzeln

GPQ gesamte Pfahlwurzelquer- Summe aller Querschnittsflächen der Pfahlwurzeln der drei schnittfläche Sektionen

GQF Gesamtwurzelquerschnitt- Summe aller Wurzelquerschnittflächen der drei Sektion eines fläche Wurzelsystems

Grobwurzeln (KÖSTLER et al Grobwurzeln im engeren Sinne 5-20 mm 1968) Derbwurzeln 20-50 mm Starkwurzeln $>50 \mathrm{~mm}$

GVA $=$ gesamte vertikale Wur- Summe aller vertikalen Wurzeln in ihrer Anzahl, über $45^{\circ}$ in zelanzahl die Tiefe gehend 
GVQ = gesamte vertikale Wur- Summe aller vertikalen Wurzelquerschnittflächen, über $45^{\circ}$ zelquerschnittfläche in die Tiefe gehend

Kronenparameter

Kronengewicht, Astgewicht, Blattgewicht, Ästchengewicht, Kronenansatz, Kronenlänge, Kronenprozent

Maximale Durchwurzelungstie- Durchwurzelungstiefe der Einzelwurzel eines Wurzelsysfe

tems, die am tiefsten wurzelt $(\mathrm{cm})$

Mittlere Gesamtwurzeltiefe

Mittelwert aller Einzelwurzeltiefen eines Wurzelsystems

Richtungswechselnde Wurzeln

Wurzeln, die in der ersten Sektion in die horizontale oder vertikale Richtung wachsen, in der weiteren Entfernung vom Stamm ihren ursprünglichen Verlauf jedoch ändern

Stammbasis

Mittelachse des Wurzelstocks

WQF Wurzelquerschnittfläche

Nach der Formel d1/2*d2/2*(П) berechnete Querschnittfläche einer Wurzel des Gesamtwurzelsystems

Wurzelast

Tritt eine Verzweigung der Wurzel vor der ersten Sektion auf, so wird dieser sich verjüngende Teil als "Wurzelast" bezeichnet (analog zu Stamm: und alles was davon abgeht, ist ein Ast)

Wurzellänge

Länge des von der Stammbasis abgehenden Wurzelastes

Wurzelstock

Zentralpartie des Wurzelsystems, d. h. dicht unter dem Stamm im Boden befindlicher Teil des Wurzelsystems, deren Basis der Ausgangspunkt für die horizontalen und vertikalen Wurzelmessungen ist

Wurzelsystem

Dreidimensionaler, halbkreisförmiger Wurzelkörper bis zu $100 \mathrm{~cm}$ horizontaler und $90 \mathrm{~cm}$ vertikaler Entfernung von der Stammbasis (Fichte, Kiefer, Buche), Eiche $70 \mathrm{~cm}$ horizontal und vertikal 


\section{Literaturverzeichnis}

AвE, K.; Iwamato, M. 1986: An evaluation of tree-root effect on slope stability by tree-root strength. Journal of the japanese forestry society $68,505-510$.

Aberts, R.; Berendse, F.; KLerK, N. M.; BaKKer, C. 1989: Root production and root turnover in two dominant species of wet heathlands. Oecologia 81, 374-378.

AG BODENKUNDE 1982: Bodenkundliche Kartieranleitung. 4. Auflage, Stuttgart, Schweizerbart, $392 \mathrm{~S}$.

Aldinger, E.; Seemann, D.; KonNerT, V. 1996: Wurzeluntersuchungen auf Sturmwurfflächen 1990 in Baden-Württemberg. Mitteilungen des Vereins für Forstliche Standortskunde und Forstpflanzenzüchtung 38, 11-25.

ARBEITSGEMEINSCHAFT FORSTEINRICHTUNG 1996: Forstliche Standortaufnahme - Begriffe, Definitionen, Einteilungen, Kennzeichnungen, Erläuterungen. Arbeitskreis Standortkartierung 5. Auflage, Enching bei München, IHW Verlag.

ARbeitskreis Standortskartierung DER ARbEITSGEMEINSCHAFT FORSTEINRICHTUNG 1985: Forstliche Wuchsgebiete und Wuchsbezirke in der Bundesrepublik Deutschland. Münster-Hiltrup, Landwirtschaftsverlag GmbH, 29-31.

ATGER, C; EDELIN, 1993: Premières donées sur l'architecture comparée des systèmes reacinaires et caulinaires. Can. Journal Bot. 72, 963-975.

BaSSAM, N. E.; SOMmer, C. 1980: Eine Methode zur in situ-Ermittlung der Leistungsfähigkeit des Wurzelnetzes von Genotypen. Zeitschrift für Acker- und Pflanzenbau 149, 191-397.

BAUMHAUER, H. 1996: Verjüngung durch Saat. Ein Beitrag zur Kostensenkung. Allgemeine Forstzeitschrift / Der Wald 51, 1192-1194.

BAZZAZ, F. A. 1997: Allocation of ressources in plants: State of the science and critical questions. In: BAZZAZ, F. A.; GrACE, J. (Eds.). Plant ressource allocation, San Diego u.a., Academic Press, 1-37.

Becker, G.; Hoffmann, R.; KREBS, M. 1988: Einsatz eines Rhizoskops zur Beurteilung eines veränderten Wurzelwachstums als Folge der Befahrung von Waldböden. Allgemeine Forstzeitschrift 43, 866-868.

BEHLEN, T. 1996: Vitalität und Wachstum eines Buchenjungwuchses unter dem Einfluß von Birken-Sukzessionsvorwald im Hessischen Forstamt Alsfeld. Fachhochschule Hildesheim-Holzminden, Fachbereich Forstwirtschaft und Umweltmanagement Göttingen. Diplomarbeit. 
BIBELRIETHER, H. 1962: Wurzeluntersuchungen an Tannen und Eichen in Mittelschwaben. Forstwissenschaftliches Centralblatt 18, 230-248.

BIBELRIETHER, H. 1964: Unterschiedliche Wurzelbildung bei Kiefern verschiedener Provenienz. Forstwissenschaftliches Centralblatt 83, 129-139

BIGING, G. S.; DobBERTIN, M. 1992: A comparison of distance-dependent competiton measures for height and basal area growth of individual conifer trees.

Forest Science 38 (3), 695-720

BIRKE, M. 1997: Wuchsdynamik und qualitative Differenzierung einer Bergahorndickung mit Birkenkonkurrenz. Fachhochschule Hildesheim-Holzminden, Fachbereich Forstwirtschaft und Umweltmanagement Göttingen. Diplomarbeit.

BöHM, W. 1979: Methods of studying root systems. Ecological Studies 33. Berlin Heidelberg New York, Springer Verlag.

BöHM, W.; KöPKE, U. 1977: Comperative root investigations with two profile wall methods. Zeitschrift für Acker- und Pflanzenbau 144, 297-303.

BOMMER, S. 1998: Untersuchung zur Konkurrenzsituation in Buchen - Douglasien - Jungbeständen im Kreisforstamt Farchau. Fachhochschule Hildesheim-Holzminden, Fachbereich Forstwirtschaft und Umweltmanagement Göttingen. Diplomarbeit

Bonnemann, A 1939: Der gleichaltrige Mischbestand von Kiefer und Buche. Mitteilungen aus Forstwirtschaft und Forstwissenschaft 10, 439-483.

Bonnemann, A. 1970: Zum Anbau der Fichte auf staunassen Böden. Forstarchiv 41, 217-221

BORCHERT, R. 1973: Simulation of rythmic tree growth under constant conditions. Physiological Plant 29, 173-180. Zitiert in: RolOFF, A und RöMER 1989, Beziehungen zwischen Krone und Wurzel bei der Rotbuche (Fagus Sylvatica L.). Allgemeine Forstund Jagdzeitung 160 (9/10); 200-205.

Bosch, A. L. 1984: A new root observation method. The perforated soil system. Acta Oecologica; Oecologia plantarum 5 (1), 61-74.

Box, J. E. jr. 1996: Use of minirhizotrans to study wheat rooting. In: PERSSON, H.; BAITULIN, I. O. (eds.), Plant root systems and natural vegetation. Acta phytogeogr. Suec. 81, 109-112.

BRAEKKe, F. H., KozlowsKI, T. T. 1977: Distribution and growth of roots in Pinus resinosa and Betula papyrifera stands. Medd. Nor. Inst. Skogfork. 33, 10, 439-451.

BRECKLE, S. W. 1996: Growth of tree roots under heavy metal (PB)-stress. In PERSSON, H.; Baitulin, I. O. (eds.) Plant root systems and natural vegetation. Acta phytogeogr. Suec. 81, 39-43. 
Brown, G. R.; ThILENIUS, J. F. 1977: A tool and method for extracting plant-root-soil cores on remote sites. J. Range Manag. 30, 72-74.

BRown, J. M. B. 1955: Ecological investigations: Shade and growth of oak seedlings. Rep. For. Res. For. Comm. 24-25. Zitiert in: RöHRIG, E. 1966, Die Wurzelentwicklung der Waldbäume in Abhängigkeit von den ökologischen Verhältnissen. Forstarchiv 37 (10), 217-229, 237-249.

BRÜCKNER, E,.; JAHN, R. 1932: Über die Wurzelausbildung verschiedener Holzarten in Böden des ostthüringischen Buntsandsteingebietes. Tharandter Forstliches Jahrbuch 83, 150-170.

BURGER, H. 1930: Bodenveränderung und Wurzelbildung. Schweizerische Zeitschrift für Forstwesen 2, 67-71.

BurSCHEL, P.; HuSS, J. 1964: Die Reaktion von Buchensämlingen auf Beschattung. Forstarchiv 35, 225-233. Zitiert in: RÖHRIG, E. 1966, Die Wurzelentwicklung der Waldbäume in Abhängigkeit von den ökologischen Verhältnissen. Forstarchiv 37 (10), 217-229, 237-249

Burschel, P.; SChMaltz, J. 1965A: Untersuchungen über die Bedeutung von Unkraut und Altholzkonkurrenz für junge Buchen. Forstwissenschaftliches Centralblatt 84, 230243.

Burschel, P.; SChMaltz, J. 1965B: Die Bedeutung des Lichtes für die Entwicklung junger Buchen. Allgemeine Forst- und Jagdzeitung 136, 193-210.

Burschel, P.; Huss, J.; Kalbhenn, R. 1964: Die natürliche Verjüngung der Buche. Schriftenreihe der forstlichen Fakultät Göttingen 34, 186 S.

BüSGEN, M.; MüNCH, E. 1927: Bau und Leben unserer Waldbäume. 3. Auflage, Jena. Fischer

BÜTTNER, V.; LEUSCHNER, C. 1994: Spatial and temporal patterns of fine root abundance in a mixed oak/beech forest. Forest Ecology and Management 70 (1-3), 11-21.

Carley, H. E.; Watson, R. D. 1966: A new gravimetric method for estimating root surface areas. Soil sci. 5, 589-291.

CASTLE, W. S. 1978: Citrus root systems: Their structure, function, growth, and relationship of tree performance. Proc. Int. Soc. Citriculture 3, 62-69. Zitiert in: RolOFF, A. und RÖMER 1989, Beziehungen zwischen Krone und Wurzel bei der Rotbuche (Fagus Sylvatica L.). Allgemeine Forstzeitschrift 160 (9/10), 200-205. 
Chapman, K.; WitTaker, J. B.; Heal, O. W. 1986: Metabolic and Faunual Activity in Litters of Tree Mixtures Compared with Pure Stands. Agriculture, Ecosystems and Environment 24, 33-40. Zitiert in: BROWN, A. H. F. 1992 Functioning of mixed species stands at Gisburn, NW- England. In: CANNELl, M. G. R.; Malcom, D. C.; Robertson, P. A. (eds), The ecology of mixed - species stands of trees. Special Publication 11 of the British Ecological Society, Blackwell Scientific Publications; Oxford, $125-150$.

Cheng, W.; Coleman, D.; Box, J. E. JR. 1991: Measuring root turnover using the minirhizotron technique. Agricalture Ecosystem Environment 34, 261-267.

Colin-Belgrand, M.; RAnger, J.; D'Argouges, S. 1993: Internal nutrient cycling in Castanea sativa wood: a dynamic study of a stand chronosequence. Acta-Oecologica 14 (5), 653-680; 44 ref..

CouTTS, M. P. 1983: Root architecture and tree stability. Plant and Soil 56, 171-188.

DHote, J. F; Mohren, G. M. J. 1994: Hypotheses about competition for light and water in even-aged common beech (Fagus silvatica L.). Forest Ecology and Management $69,1-3,219-232$.

DO-HyUNG LEE 1998: Architektur der Wurzelsysteme von Fichten (Picea abies L) auf unterschiedlich versauerten Standorten. Berichte des Forschungszentrums Waldökosysteme, Reihe A, 153.

DRESCHER, W. 1959: Aus der Bestandes- und Ertragsgeschichte von Beständen des südlichen Hochschwarzwaldes. Schriftenreihe der Landesforstverwaltung Baden-Württemberg 19.

DreXHAGE, M. 1994: Die Wurzelentwicklung 40 jähriger Fichten (PICEA ABIES [L]) in der Langen Bramke (Harz). Berichte des Forschungszentrums Waldökosysteme. Reihe A, 111 .

EGLI, S.; KäLIN, I. 1991: Root window technique for in vivo observation for ectomycorrhiza on forest trees. In: NORRIS, J. R.; REED, D. J. (eds), Methods on microbiology, Vol. 23. New York, Academic Press, 423-433.

EhWAld, E.; Grunert, F.; SChulz, W.; VetTerlein, E. 1961: Zur Ökologie von KiefernBuchen-Mischbeständen. Archiv für Forstwesen 10 (4-6), 397-416.

EICHHORN, J. 1992: Wurzeluntersuchungen an sturmgeworfenen Bäumen in Hessen. Forst und Holz 47 (18), 555-559.

Ellern, S. J.; HARPER, J. L.; SATAR, G. R. 1970: A comparative study of the distribution of the root of Avena futua and Avena strigosa in mixed stands using a $14 \mathrm{C}$ laballing technique. Journal of ecology 58, 865-868. 
FABRICIUS, L. 1929: Neue Versuche zur Feststellung des Einflusses von Wurzelwettbewerb und Lichtentzug des Schirmstandes auf den Jungwuchs. Forstwissenschaftliches Centralblatt 51, 477-506.

FEICHT, H.; GEYER, R. 1993: Untersuchungen zur Wurzelentwicklung natürlich verjüngter/gesäter und gepflanzter Eichen auf im Unterboden verdichteten Standorten. In: Bericht über die Arbeitstagung "Pflanztechnik und Wurzelentwicklung" der Bayerischen Forstlichen Versuchs- und Forschungsanstalt, 48-60.

Fernandez, A.; Paruela, J. M. 1988: Root systems of two patagonian shrubs: A quantitative description using a geometrical method. Journal of Range Management 41, 220-223.

Fiedler, H. J.; Hunger, W.; ZANT, R. 1963: Untersuchungen über die Bodendurchwurzelung der Fichte. Archiv für Forstwesen 12, 1214-1223.

FOGEL, R 1985: Roots as primary producers in below-ground ecosystems. Special Series of the British Ecological Society 4, 22-36.

FölSTER, H., DegenhaRD, M.; Flor, T.; LuX, M. 1991: Untersuchungen zur Tiefendurchwurzelung und Durchwurzelungsintensität auf Braunerde-Pseudogleyen im Vorderen Hunsrück in Abhängigkeit von Baumart und Bestandesstrukturparametern. In: Mitteilungen aus der Forstlichen Versuchsanstalt Rheinland-Pfalz 19, 91-106.

FritzsCHE, K. 1933: Sturmgefahr und Anpasssung. (Physiologische und technische Fragen des Sturmschutzes). Tharandter forstliches Jahrbuch 84, 1-94.

GANNßEN, R. H. 1934: Untersuchungen an Buchenstandorten Nord- und Mitteldeutschlands. Zeitschrift für Forst- und Jagdwesen 36, 225-249, 359-394, 472-494, 583-606.

GAYER, K. 1867: Über forstliche Versuchsstationen insbesondere in Bayern. Monatsheft für Forst- und Jagdwesen, 201-213.

GENSSLER, H. 1959: Veränderungen von Boden und Vegetation nach generationsweisen Fichtenanbau. Universität Göttingen, Forstliche Fakultät, Dissertation.

GiJsman, A. J.; Floris, J.; VAn NoORdWiJK, M.; Brouwer, G. 1991: An influtable minirhizotron system for root observations wirh improves soil/tube contact. Plant and soil 134, 261-269.

GRACE, J. 1997: Toward models of resource allocation by plants. In: BAZZAZ, F. A.; GRACE, J. (eds.), Plant resource allocation. San Diego u.a., Academic Press, 279-291.

Grier, C. C.; Vogt K. A.; KeYeS, M. R.; Edmonts, R. L. 1981: Biomass distribution and above- and below-ground production in young and mature Abies amabilis zone ecosystems in the Washington Cascades. Can. Journal of Forest Research 11, 155-167. 
GrOSSKOPF, W. 1950: Bestimmung der charakteristischen Feinwurzelintensitäten in ungünstigen Waldprofilen und ihre ökologische Auswertung. Mitteilungen der Bundesanstalt für Forst- und Holzwirtschaft 11, 1-19.

GrUBER, F. 1992: Mündliche Mitteilungen. Zitiert in: Do-HYUng LEE (1997). Architektur der Wurzelsysteme von Fichten (Picea abies [L]) auf unterschiedlich versauerten Standorten. Berichte des Forschungszentrums Waldökosysteme, Reihe A, 153, 74.

GrubER, F. 1992: Dynamik und Regeneration der Gehölze. Berichte des Forschungszentrums Waldökosysteme. Reihe A, 86 / Teil 1.

HÄHNE, K. 1991: Der Einfluß von Gräsern- und Gehölzwurzeln auf die Scherfestigkeit von Böden und damit auf die Standsicherheit von Hängen und Böschungen. Technische Universität Berlin, Institut für Landschaftsbau, Dissertation.

HARLEY, J. L.; WAID, J. S. 1955: The effect of light on the roots of beech and its surface population. Plant and soil, 96-112. Zitiert in: Röhrig, E. 1966, Die Wurzelentwicklung der Waldbäume in Abhängigkeit von den ökologischen Verhältnissen. Forstarchiv 37 (10), 217-229, 237-249[M21].

HARTKOPF, A. 1994: Zur Konkurrenzwirkung von Weichlaubhölzern in einem Fichtenjungwuchs. Universität Göttingen Forstwissenschaftlicher Fachbereich, Institut für Waldbau Abt. I,. Diplomarbeit.

HAuSKELleR-BulleRJAHN, K. 1997: Wachstum junger Eichen unter Schirm. Berichte des Forschungszentrums Waldökosysteme, Reihe A, 147.

HEGYI, F. 1974: A simulation model for managing jack-pine stands. In Growth models for tree and stand simulation, Rapporter ou uppsatser, Institutionen för skogsproduktion, Skogshögskolan, Stockholm, 74-76.

HENDRIKS, C . M. A., BiANCHI, F. J. J. A. 1995: Root density and root biomass in pure and mixed forest stands of douglas fire and beech. Netherlands Journal of agricultural Science 43 (3), 321-331.

HILF, H. H. 1927: Studien über die Wurzelausbreitung von Fichte, Buche und Kiefer in geschlossenern älteren Beständen, insbesondere auf Sandböden. Eberswalde, Dissertation, $121 \mathrm{~S}$.

Hochtanner, G.; SeitscheK, O. 1964: Wuchsleistungen von Kiefernbeständen auf Meliorierungsflächen nach dem Bodenwöhrer Verfahren. Forstwissenschaftliches Centralblatt $83,1-22$.

HofFmanN, R. 1939: Vergleichenden Untersuchungen über die Wurzeltracht forstlicher Kleinpflanzen. Ludwig-Maximilians-Universität München, Dissertation.

HolmeS, M. J.; REED, D. D. 1991: Competition indices for mixed species northern hardwoods. Forest Science 37 (5), 1338-1349. 
ITOH, S.; BARBER, S. A. 1983: Phosphorus uptake by six plant species as related to root hairs. Agron. Journal 75, 457-461.

JAHN, R.; SCHEFFOLD, K.; HAUCK, U. 1971: Wurzeluntersuchungen von Waldbäumen in Baden-Württemberg. Schriftenreihe der Landesforstverwaltung BadenWürttembergs 33, $120 \mathrm{~S}$.

JENIK, J. 1957: Das Wurzelsystem der Stieleiche und Traubeneiche. Rozpravy Ceskoslovenske Akademie Ved.. Zitiert in: KöSTLER et al 1968 Die Wurzeln der Waldbäume in Mitteleuropa. Hamburg Berlin, Paul Parey Verlag.

JARVIS, P. G. 1964: The adaptability to light intensity of seedlings of Quercus petraea (Matt.) Liebl. Journal of ecology, 545-568. Zitiert in: RÖHRIG, E. 1966 Die Wurzelentwicklung der Waldbäume in Abhängigkeit von den ökologischen Verhältnissen. Forstarchiv 37 (10), 217-229, 237-249.

JoHnSON, P. S. 1993: Sources of oak reproduction. In: LOFTIS, D.L.; MCGEE, C.E. (eds.), Oak regeneration: Serious problems, practical recommendations Symposium oak regeneration, Knoxville, Tennessee, Sept. 8-10, 1992; Generale technical report, SE 84, 112-131.

JÜTTNER, O. 1954: 70 Jahre Heideaufforstung. Bremen, Walter Dorn Verlag.

KALELA, E.K. 1955: Über Veränderungen in den Wurzelverhältnissen der Kiefernbestände im Laufe der Wachstumsperiode. Acta For. Fenn. 65, 1-41.

KALININ, M. I. 1978: Modellierung der Waldbestände (russ.). Lwow, Wistscha Schkola Verlag, $205 \mathrm{~S}$.

KALININ, M. I. 1983: Ausbildung der Wurzelsysteme von Bäumen (russ.). Moskwa, Lesnaja Promsylennost.

KARIZUMI, N. 1968: Esimation of root biomass in forests by the soil block sampling. In GHILARAV, M. S.; Kovdal, V. A. (eds), Methods of productivity studies in root systems and rhizosphere organism. International Symposium 1968, USSR Leningrad, Nauka, 79-86.

Kaussmann, B.; SCHIEWER, U. 1989: Funktionelle Morphologie und Anatomie der Pflanzen. Jena, Fischer Verlag.

KENNEL, R. 1966: Soziale Stellung, Nachbarschaft und Zuwachs. Forstwissenschaftliches Centralblatt 85, 193-204.

KEYES, M. R.; GRIER, C. C. 1981: Above- and below-ground net production in 40 years old douglas fir stands on low and hight productivity sites. Canadian Journal of Forest Research 11, 599-605. 
KleinsChmit, J. R. G.; Kremer, A.; RolofF, A. 1995 : Sind Stieleiche und Traubeneiche zwei getrennte Arten? Allgemeine Forstzeitschrift / Der Wald 50 (26), 1453-1456.

KoKKonen, P. 1929: Beobachtungen über das Wurzelsystem der Kiefer im Moorböden. Acta for finn. 25, 1-21.

KöNIG, A; MößMER, R., BäUMLER, A. 1995: Waldbauliche Dokumentation der flächigen Sturmschäden des Frühjahrs 1990 in Bayern und meteorologische Situation zur Schadenszeit. Berichte aus der bayerischen Landesanstalt für Wald- und Forstwirtschaft (LWF) 2, 19-25, 47-70.

Köstler, J. N.; BRÜCKNer, E; Bibelriether, H. 1968: Die Wurzeln der Waldbäume in Mitteleuropa. Hamburg-Berlin, Paul Parey Verlag.

Kozlowski, T. T.; Pallardy, S. G. 1997: Growth control in woody plants. San Diego u.a., Academic Press.

KozlowSKI, T. T. 1972: Seed biology, Vol. II. Academic Press, New York-London.

KRAHL-URBAN, J. 1951: Waldfeldbau in einem Mittelgebirgsrevier. Schriftenreihe der Forstlichen Fakultät der Universität Göttingen 1.

KRAMER, H. 1975: Erhöhung der Produktionssicherheit zur Förderung einer nachhaltigen Fichtenwirtschaft. Forstarchiv 46, 9-13

KrAMER, H.; GuSSONE, H. A.; SCHOBER, R. 1988: Waldwachstumslehre. Hamburg - Berlin, Paul Parey Verlag, 374 S.

KrAMER, H.; AKCA, A. 1987: Leitfaden für Dendrometrie und Bestandesinventur. Frankfurt am Main, J. D. Sauerländer's Verlag, 287 S.

KraUSS, G. 1938: Wald und Boden. In: Raumforschung und Raumordnung 2 (11/12), 592596.

KRAUSS, G.; WoBST, W.; GÄrTner, G. 1935: Humusauflage und Bodendurchwurzelung im Eibenstocker Granitgebiet. Tharandter Forstliches Jahrbuch 85, 17-51.

KREUTZER, K. 1961: Wurzelbildung junger Waldbäume auf Pseudogleyböden. Forstwissenschaftliches Centralblatt 80, 356-392.

KrONAUER, H. 1990: Ausmaß der Sturmschäden 1990 und Maßnahmen zur Bewältigung. Allgemeine Forstzeitschrift 45 (17/18), 434-437.

LAITAKARI, E. 1929A: Die Wurzelforschung in ihrer Beziehung zur praktischen Forstwissenschaft, Acta for. Fenn. 33, 1-380. 
LAITAKARI, E. 1929B: The root system of pine - A morphological investigation. Acta for. Fenn. 33, 307-380.

LEIBUNDGUT, H. 1975: Untersuchungen über die gegenseitige Beeinflussung verschiedener Baumarten. Schweizerische Zeitschrift für Forstwesen 127, 766-771.

LEMKE, K. 1956: Untersuchungen über das Wurzelsystem der Roteiche auf diluvialen Standortsformen. Archiv für Forstwesen 5, 8-45, 161-202.

LiChTENEGGER, E. 1985: Die Ausbildung der Wurzelsysteme krautiger Pflanzen und deren Eignung für Böschungssicherung auf verschiedenen Standorten. In PFLUG, E. (Hrsg). Wurzelwerk und Standsicherheit von Böschungen und Hängen. Aachen, SEPIA Verlag, 63-82.

LIESE, J. 1926: Beiträge zur Kenntnis des Wurzelsystems der Kiefer (Pinus sylvestris) nebst anderen Baumarten. Zeitschrift für Forst- und Jagdwesen 58, 129.

Long, T. J.; JoneS, R. H. 1996: Seedling growth strategies and seed size effects in fourteen oak species native to different soil moisture habitats. Trees (- Structure and Function -) $11(1), 1-8 ; 54$.

LYR, H. 1994: Effect of root temperature on growth parameters of QUERCUS ROBUR compared to other tree species. IUFRO-Symposium, Environmental constraints and oaks. Nancy.

MACKENTHUN, G. 1990: Vergleichende Ökologische Untersuchungen eines Fichten / Buchenmischbestandes und verschiedener Reinbestände in Westerhof, Harzvorland. Universität Göttingen, Forstwissenschaftlicher Fachbereich, Geobotanisches Institut / Institut für Waldbau Abt. I. Diplomarbeit.

MARSCHNER, H. 1986: Mineral nutrition of higher plants. London, Academic press. Zitiert in: Abschlußbericht 1989-1993 zum BMFT- Forschungsvorhaben. Stabilitätsbedingungen von Waldökosystemen Teil B, 77.

MaYer, A. M.; PolJakoff - MaYber, A. 1975: The germination of seeds. Oxford - New York, Pergamon Press.

MCGEE, C. E.; LOFTIS, D. L. 1993: Oak regeneration: A summary: Serious problems, practical recommendations; Symposium Oak regenaration, Knoxville, Tennessee, Sept. 810, 1992, LofTIS, D. L.; McGeE, C. E. Gen. Tech. Rep., SE 84, Asheville, N. C., 316-319.

MCKAY, H. M.; MALCOM, D. L.1988: A comparison of the fine root component of a pure and a mixed coniferous stand. Canadian Journal of Forest Research 18 (11), 1416-1426.

MeIWES, K. J. 1984: Chemische Untersuchungsverfahren für Mineralböden, Auflagehumus und Wurzeln. Berichte des Forschungszentrums Waldökosysteme 7, 1-67. 
MelzER, E. W. 1962: Die stochastischen Beziehungen zwischen Sproß- und Wurzelsystem des Baumes. Archiv für Forstwesen 11 (7), 822-838.

MiaO-ShiLi; MiaO-SL 1995: Acorn mass and seedling growth in Quercus rubra in response to elevated CO2. Journal of Vegetation Science, 6 (5), 697-700; 17 ref.

MoIR, W. H., BaChelaRd, E. P. 1969: Distribution of fine roots in three Pinus radiata plantations near Canberra, Australia. Journal of Ecology 50, 658 - 663.

Morosow, G. F. 1928: Die Lehre von den Waldtypen; Neudamm. Zitiert in: KöSTLER et al 1968, Die Wurzeln der Waldbäume in Mitteleuropa. Hamburg - Berlin, Paul Parey Verlag.

MößMER, R. 1997: Untersuchung der Sturmschäden des Jahres 1990 in Bayern. Bayerische Landesanstalt für Wald und Forstwirtschaft, Bericht zur 1. Wiederholungsaufnahme "Pflanzverfahren und Pflanztechnik".

MulLIN, R. E. 1963: Growth of white spruce in the nursery. Forest Science 9, 68.

MURACH, D. 1989: Natural and anthropogenic stress in spruce and beech ecosystems in the Solling project. In: Abschlußbericht 1989-1993 zum BMFT-Forschungsvorhaben. Stabilitätsbedingungen von Waldökosystemen Teil B, 69-78.

NiELSEN, C. N. N. 1990: Einflüsse von Pflanzenabstand und Stammzahlhaltung auf Wurzelform, Wurzelbiomasse, Verankerung sowie auf die Biomasseverteilung im Hinblick auf die Sturmfestigkeit der Fichte. Frankfurt am Main, J. D. Sauerländer's Verlag, Schriften aus der Forstlichen Fakultät der Universität Göttingen und der Forstlichen Versuchsanstalt 100.

NIELSEN, C. N. N. 1995: Report of project "Development of roots and root/ shoot-ratios. Air3CT93-1269, unveröffentlicht.

Ovington, J. D.; MCRAe, C. 1960: The growth of seedlings of Quercus petraea. Journal of Ecology, 639-646. Zitiert in: RöHRIG, E. 1966 Die Wurzelentwicklung der Waldbäume in Abhängigkeit von den ökologischen Verhältnissen. Forstarchiv 37 (10), 217-229, 237-249.

Pellinen, P. 1986: Biomassenuntersuchungen im Kalkbuchenwald. Universität Göttingen, Forstwissenschaftler Fachbereich. Dissertation.

Persson, H. 1979: Fineroot production, mortality and decomposition in forest ecosystems. Vegetatio 41, 101-109.

Persson, H. 1980: Spatial disribution of fine- root growth, mortality and decomposition in a young Scots pine stand in central Sweden. Oikos 34, 77-87.

PFEIL, W. 1860: Die deutsche Holzzucht. Leipzig. 
Pijnenborg, W. M.; Lie, T. A.; Zehnder, A. J. B. 1990: Nodulation of lucerne (Medicago sativa L.) in an acid soil: $\mathrm{pH}$ dynamics on the rhizosphere of seedlings growing in rhizotrons. Plant and soil 126, 161-168.

Polomski, J; KuHN, N. 1998: Wurzelsysteme. Bern - Stuttgart - Wien, Paul Haupt Verlag.

PUHE, J. 1994: Die Wurzelentwicklung der Fichte (Picea abies [L.] Karst.) bei unterschiedlichen chemischen Bodenbedingungen. Berichte des Forschungszentrums Waldökosysteme, Göttingen, Reihe A, 108.

RACHTEJenKo, J. N. 1952: Die Wurzelsysteme der Baum- und Straucharten. Golesbumisdat, Moskau. Zitiert in: MELzER, E. W. 1962, Die stochastischen Beziehungen zwischen Sproß- und Wurzelsystem des Baumes. Archiv für Forstwesen 11 (7), 822-838.

RAPP, C.; JENSCHKE , G. 1994: Acid deposition and ectomycorrhizal symbiosis: Field investigations and causal relationships. In: GODBOLD, D. L. and HÜTTERMAN, A. (Hrsg.) Effects of acid rain on forest processes. New York, Wiley - Liss, 183-230.

RASPE, S. 1992: Biomasse und Mineralstoffgehalte der Wurzeln von Fichtenbeständen (Picea abies [L] Karst) des Schwarzwaldes und Veränderungen nach Düngung. Freiburger bodenkundliche Abhandlungen 29.

Raven, P. H.; Evert; R.F.; Curtis, H. 1988: Biologie der Pflanzen. 2., verbesserte Auflage, Berlin New York, Walter de Gruyter.

RICE, K. J.; GORdON, D. R.; HARDISON, J. L.; WelKER, J. M. 1993: Phenotypic variation in seedlings of a 'keystone' tree species (Quercus douglasii): the interactive effects of acorn source and competitive environment. Oecologia 96 (4), 537-547.

RöHRIG, E. 1958: Die Anzucht von Forstpflanzen in Nadelstreubeeten. Schriftenreihe der forstlichen Fakultät und Mitteilungen der Niedersächsischen forstlichen Versuchsanstalt Göttingen 22.

RöHRIG, E. 1966: Die Wurzelentwicklung der Waldbäume in Abhängigkeit von den ökologischen Verhältnissen. Forstarchiv 37 (10), 217-229, 237-249.

RöHRIG, E. 1976: Einfluß unterschiedlicher Saattiefe auf die Entwicklung von einjährigen Eichen-Sämlingen. Der Forst- und Holzwirt 31, 119-121.

RöHrig, E.; Gussone, H. A. 1990: Waldbau, Alfred Dengler 2. Band. Baumartenwahl, Bestandesbegründung und Bestandespflege. Hamburg und Berlin, Paul Parey Verlag.

RolOFF, A.; BÄrTELS, A. 1996: Gartenflora Bd. 1 Gehölze. Eugen Ulmer Verlag; S. 694.

ROLOFF, A.; RöMER, H.-P. 1989: Beziehungen zwischen Krone und Wurzel bei der Rotbuche (Fagus sylvatica L.). Allgemeine Forst- und Jagdzeitung 160 (9/10), 200-205. 
Rothe, A. 1997: Einfluß des Baumartenanteils auf Durchwurzelung, Wasserhaushalt, Stoffhaushalt und Zuwachsleistung eines Fichten - Buchen - Mischbestandes am Standort Höglwald. Forstliche Forschungsberichte München 163.

ROZSNYAY, Z. 1979: Forstgeschichtliche Betrachtungen zur Entstehung der KRAFT'schen Baumklasse. Allgemeine Forst- und Jagdzeitung 150, 65-72.

RYSAVI, T.; RolOFF, A. 1994: Ursachen der Vereschung in Mischbeständen und Vorschläge zu ihrer Vermeidung. Forst und Holz 49, 392-395.

SACHS, L. 1978: Angewandte Statistik. 5. Auflage, Berlin - Heidelberg - New York, Springer.

SANTANTONIO, D. 1989: Dry-matter portitioning and fine-root production in forests-new approaches to a difficult problem. In: PEREIRA J. S. and LANDSBERG J. J. (eds.), Biomass Production by fast - growing trees, 57-72.

SATTELMACHER, B.; KLOTZ, F.; MARSCHNER, H. 1983: Bestimmung von Wurzeloberflächen mit Hilfe von Schnellmethoden. In: BÖHM, W.; KUTSCHERA, L; LiCHTENEGGER, E. (Hrsg.), Wurzelökologie und ihre Nutzanwendung. Internationales Symposium vom 27.-29.9.1982, Bundesanstalt für alpenländische Landwirtschaft, Gumpenstein, A-Irdning, 95-98.

SCAMONI, A. 1950: Waldbauliche Untersuchungen auf grundwassernahen Talsanden. Berlin.

SchefFer, F., Schachtschabel, P. 1992: Lehrbuch der Bodenkunde. 13. Auflage, Stuttgart, Ferdinand Enke Verlag.

SCHIECHTLE, H. M. 1983: Pflanzen als Mittel zur Bodenstabilisierung. In BöHM, W.; KUTSCHERA, L.; LiCHTENEGGER, E. (Hrsg.), Wurzelökologie und ihre Nutzanwendung. Internationales Symposium vom 27.-29.9.1982, Bundesanstalt für alpenländische Landwirtschaft, Gumpenstein, A-Irdning, 703-708.

Schlichter, T. M.; Ploeg, R. R. VAn DeR; Ulrich, B. 1983: A simulation model on the water uptake of a beech forest: Testing variations in root biomass and distribution. Zeitschrift für Pflanzenernährung und Bodenkunde 146, 725-735.

SchmaltZ, J.; Merkel, H. 1995: Waldbaugrundlagen. Skriptenreihe der Fachhochschule Hildesheim - Holzminden, Fachbereich Forstwirtschaft Göttingen, 5.

SCHMID-HAAS, P.; BACHHOFEN, H. 1991: Die Sturmgefährdung von Einzelbäumen und Beständen. Schweizerische Zeitung für Forstwesen 142, 477-504.

SCHMIDT - VogT, H.; GÜRTH, P. 1977: Eigenschaften von Forstpflanzen und Kulturerfolg. II. Mitteilung; Auspflanzungsversuche mit Fichtenpflanzen verschiedener Größen und Durchmesser mit Winkel-und Lochpflanzung. Allgemeine Forst- und Jagdzeitung $148,145-157$. 
SCHMIDT-VoGT, H. 1966: Wachstum und Qualität von Forstpflanzen. 2. Auflage, München Basel - Wien, Bayerischer Landwirtschaftsverlag.

SCHMIDT-Vogt, H. 1977: Die Fichte, Bd I Hamburg - Berlin, Paul Parey Verlag.

SChMidT-Vogt, H.; WÜTherich, G.; Deichner, P. 1987: Untersuchungen zur Sturmstabilität von Fichten-Tannen Mischbeständen auf verschiedenen Standorten Süddeutschlands. Allgemeine Forst- und Jagdzeitung 158, 42-50.

SChMitz, P. Groth, A. 1984: Vergleichende Untersuchung der Vertikalwurzelentwicklung von Traubeneichen bei unterschiedlicher Begründungsart. Albert-LudwigUniversität Freiburg i. Br., Institut für Waldbau, Diplomarbeit, 96 S.

SCHOBER, R. 1975: Ertragstafeln wichtiger Baumarten. Frankfurt am Main, J. D. Sauerländer's Verlag.

SCHÜTE, G.; TAE SU, K. 1993: Vergleichende Wurzeluntersuchungen an Stecklingen, in vitro vermehrten Pflanzen, Direktsaaten und Sämlingen der Stiel- und Traubeneiche. In: Beiträge zur In: Schriften aus der Forstlichen Fakultät der Universität Göttingen und der Niedersächsischen Forstlichen Versuchsanstalt 111, 159-212.

SCHÜTZ, J. P. 1989: Zum Problem der Konkurrenz in Mischbeständen. Schweizerische Zeitschrift für Forstwesen 140 (12), 1069-1083.

SEufert, G.; Wöllmer, H; ARNDT, U; BABEL, U. 1986: Das Rhizoskop-eine Möglichkeit zur störungsarmen Beobachtung des Wurzelraumes. Allgemeine Forstzeitschrift 41 (20), 493-493.

SHAW, M. W. 1974: The reproductive characteristics of oak. MORRIS, M. G.; PERRING, F. H. (eds.) "The british oak" Botanical society of the british Isles. E. W. Classey Ltd; Faringdon, 162-181. Zitiert in: HAUSKELLER-BULLERJAHN, K. 1997, Wachstum junger Eichen unter Schirm. Berichte des Forschungszentrums Waldökosysteme, Reihe A, Bd. 147.

STEEN, E. 1983: The net stocking method for studying quantitative and qualitative variation with time of grass roots. In BöHM, W.; KuTSCHERA, L; LCHTENEGGER, E. (Hrsg) Wurzelökologie und ihre Nutzanwendung. Internationales Symposium vom 27.29.9.1982, Bundesanstalt für alpenländische Landwirtschaft, Gumpenstein, A- Irdning, 63-74.

STEINBERG, D.; FReYTAG, E. 1980: Wurzelverteilungsmessungen nach Applikation radioaktiver Tracer. Übersichtsbeitrag. Archiv für Bodenfruchtbarkeit und Pflanzenproduktion 24, 351-358.

STOKES; A.; GUITARD, D 1997: Tree root response to mechanical stress. In: The biology of root formation and development. Plenum Publishing Corp., New York, Basic Life Sci., Vol. 65, 227-236. 
StONE, E. L., KAlisz, P. J. 1991: On the maximum extent of tree roots. For. Ecol. Manag. 46, 59-102.

SuTTON, R. F. 1978: Root system development in young outplants, particulary white spruce proceedings of the root farm of planted trees Symposium, Victoria B. C., Canada-B. C. Ministry of Forests / Canadian Forestry Service, Joint Report 8, 172-185.

SzAPPANOS, A. 1969: Der Beleuchtungsgrad der Traubeneichenbestände und deren Auswirkung auf das Wachstum des Jungwuchses. Az Erdészeti és Faipari Egyetem 2, 89104

TOMÉ, M.; BuRKharT, H. E. 1986: Distance-dependent competition measures for predicting growth of individuel trees. Forest Science 35 (3), 816-831.

Ulrich, B.; PirouzPanah, D., Murach, D. 1984: Beziehungen zwischen Bodenversauerung und Wurzelentwicklung von Fichten mit unterschiedlich starken Schadsymptomen. Forstarchiv 55, 127-134.

UPCHURCH, D. R.; MCMICHAEL, B. L.; TAYLOR, H. M. 1988: Use of minirhizotrans to characterize root system orientation. Soil Science Am. J. 52, 319-323.

VATER, H. 1927: Die Bewurzelung der Kiefer, Fichte und Buche. Tharandter Jahrbuch 78, 6585.

VAvoulidou-TheOdorou, E.; BABEL, U. U. 1983: Methoden zur quantitativen Bestimmung von Fichtenfeinwurzeln. Mitteilungen der deutschen bodenkundlichen Gesellschaft 38, 783-788.

VöGTLE, H. 1985: Fichtenrein- und Fichten-Buchen-Mischbestände. Allgemeine Forstzeitschrift 40 (36), 924.

Vos, J.; GroenwaLd, J. 1983: Estimation of root densities by observation tubes and endoscope. Plant and soil 74, 295-300.

WAGENHOFF, A. 1938: Untersuchungen über die Entwicklungen des Wurzelsystems der Kiefer auf diluvialen Sandböden. Zeitschrift für Forst- u. Jagdwesen 70, 449.

WAGENKNEChT, E. 1955: Wurzeluntersuchungen und ihre Bedeutung für standortsgerechten Waldbau. Archiv f. Forstwesen 4, 397-406.

WAgenKnecht, E.; Belitz, G. 1959: Die Fichte im nordostdeutschen Flachland: Anbauwürdigkeit u. waldbauliche Behandlung. Radebeul u.a., Neumann, $121 \mathrm{~S}$.

WAGNER, S. 1994: Die Rhizoskopie-Erfahrungen mit der störungsarmen Wurzelbeobachtung. Forst und Holz 49 (10), 270-273.

WALTER, H. 1962: Grundlagen des Pflanzenlebens, Bd. I. 4. Auflage, Stuttgart. 
Weaver, J. E. 1919: The Ecological Relations of Roots. Carnegie Institution of Washington. Washington D. C., Publ. 286.

Weissen, F; Van PraAg, H. J. 1991: Root growth inhibition effects of holoorganic moder humus layer under spruce (Picea abies (L) Karst.) and beech (Fagus sylvatica L.). Plant and Soil 135, 167-174.

Whiteley, G. M., DeXTer, A. R. 1984: The behaviour of roots encountering cracks in soil. In: Plant and soil 77, 141-149.

WiedemanN, E. 1927: Der Wurzelbau älterer Waldbäume. Forstarchiv 3, 229-233.

WiEdEMANN, E. 1942: Die schlechtesten ostdeutschen Kiefernbestände. Berlin

Wiedemann, E. 1943: Der Vergleich der Massenleistung des Mischbestandes mit dem Reinbestand. Allgemeine Forst und Jagdzeitung 119, 123-132

WiEDEMANN, E. 1951: Ertragskundliche und waldbauliche Grundlagen der Forstwissenschaft. Frankfurt a. M.

WiтTICH, W. 1948: Die heutigen Grundlagen der Holzartenwahl. 2. Auflage, Hannover, Schaper, $67 \mathrm{~S}$.

WiTTKOPF, W. 1995: Wurzelintensität im Fichten-Buchen-Mischbestand im Vergleich zum Reinbestand; Ludwig-Maximilians-Universität München, Forstwissenschaftlicher Fachbereich. Diplomarbeit.

WöLLMER, H. 1987: Methodik der Rhizoskopie zur Untersuchung des Feinwurzelwachstums von Waldbäumen. Universität Hohenheim, Institut für Bodenbiologie und Standortslehre, Diplomarbeit.

Zoon, F. C.; Tienderen Van, P. H. 1990: Araoid quantitative measurement of root length anf root branching by microcomputer image analysis. Plant and soil 126, 301-308. Zitiert in: PolOMSKI, J.; KuHN, N. 1998, Wurzelsysteme. Bern - Stuttgart, Paul Haupt Verlag, 22.

ZOTH, R.; BLOCK, J. 1992: Untersuchungen an Wurzelballen sturmgeworfener Bäume in Rheinland-Pfalz. Forst und Holz 47 (18), 566-571. 


\section{Tabellenverzeichnis}

Tabelle 1: Klimadaten der Untersuchungsflächen (Arbeitskreis Standortskartierung der Arbeitsgemeinschaft Forsteinrichtung; 1985) ..................................................... 5

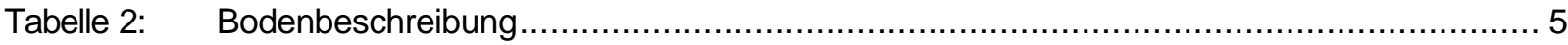

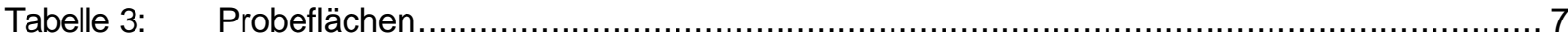

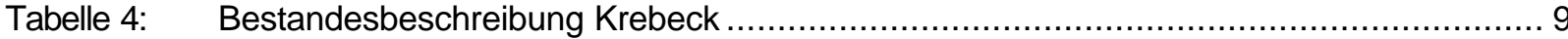

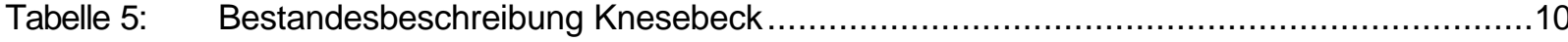

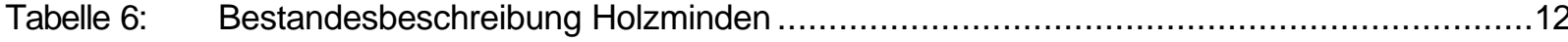

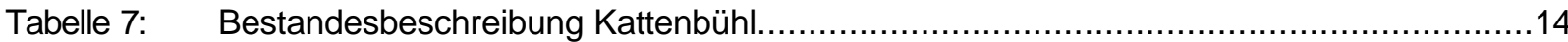

Tabelle 8: $\quad$ Größe der Radien und Schnittebenen der gemessenen Sektionen (Abstände

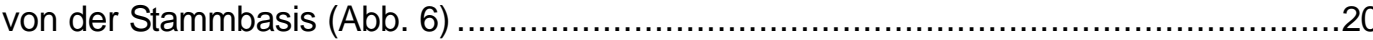

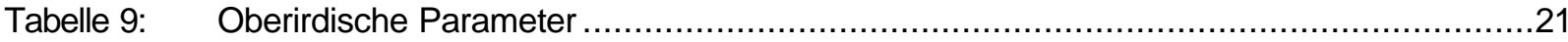

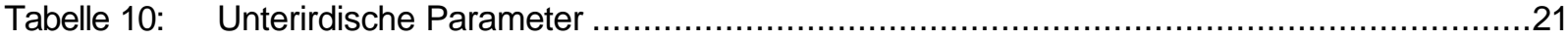

Tabelle 11: Ausgleichsfunktionen zur Bestimmung der Wurzeldurchmesser in einer beliebigen Entfernung vom Wurzelstock; $x$ = Entfernung vom Wurzelstock; $y=$ Wurzeldurchmesser.

Tabelle 12: Nach den Ausgleichsfunktionen (Tabelle 11) berechnete Wurzellängen (mittlere Einzelwurzeln der untersuchten Bestände).

Tabelle 13: Prozentualer Wassergehalt der Krone

Tabelle 14: Konkurrenzbeschreibende Daten; Holzerode, Buche und Fichte 55-65jährig; (HEGYI-Index, Anzahl der Konkurrenten, Baumabstand der nächsten drei Nachbarn zum Probebaum); der Stern * beschreibt die signifikanten Unterschiede ( $f$-Test) des HEGYI-Index $(p=0,05)$ in Richtung der Pfeile.

Tabelle 15: Konkurrenzbeschreibende Daten; Krebeck, Fichte 21-41 jährig; (HEGYI-Index, Baumabstand der nächsten drei Nachbarn zum Probebaum); der Stern * beschreibt die signifikanten Unterschiede (f-Test) des HEGYI-Index $(p=0,05)$ in Richtung der Pfeile.

Tabelle 16: Konkurrenzbeschreibende Daten; Knesebeck, Fichte und Kiefer, 31-50jährig; (HEGYI-Index, Baumabstand der nächsten drei Nachbarn zum Probebaum); der Stern * beschreibt die signifikanten Unterschiede (f-Test) des HEGYI-Index $(p=0,05)$ in Richtung der Pfeile

Tabelle 17: Konkurrenzbeschreibende Daten; Holzminden, 22 jährige Eiche (HEGYIIndex, Baumabstand der nächsten drei Nachbarn zum Probebaum); der Stern * beschreibt die signifikanten Unterschiede ( $f$-Test) des HEGYI-Index $(p=0,05)$ in Richtung der Pfeile

Tabelle 18: Konkurrenzbeschreibende Daten; Kattenbühl, 13 jährige Eiche (HEGYI-Index, Baumabstand der nächsten drei Nachbarn zum Probebaum)

Tabelle 19: $\quad$ Summe der Überragungswinkel als Maß der Konkurrenz; Holzerode, Buche und Fichte. Der Stern * beschreibt die signifikanten Unterschiede (f-Test) des Überragungswinkels $(p=0,05)$ in Richtung der Pfeile

Tabelle 20: Mittlere Probebaumparameter $\mathbf{x}$ und Standardabweichungen (sx); Holzerode, Fichte und Buche im Rein- und Mischbestand (55-65jährig). Der Stern* 
beschreibt die signifikanten Unterschiede (f-Test) des Überragungswinkels ( $p$ $=0,05)$ in Richtung der Pfeile

Tabelle 21: Mittlere Probebaumparameter $\mathbf{x}$ und Standardabweichungen (sx); Krebeck, 21-, 31- und 41 jährige Fichte im Reinbestand.

Tabelle 22: Mittlere Probebaumparameter $\mathbf{x}$ und Standardabweichungen (sx); Knesebeck, 20-50 jährige Fichte und Kiefer im Reinbestand.

Tabelle 23: Mittlere Probebaumparameter $\mathbf{x}$ und Standardabweichungen (sx); Holzminden, 22 jährige Eiche; Saat und Pflanzung. Der Stern * beschreibt die signifikanten Unterschiede (f-Test) des Überragungswinkels $(p=0,05)$ in Richtung der Pfeile

Tabelle 24: Mittlere Probebaumparameter $\mathbf{x}$ und Standardabweichungen (sx); Kattenbühl, 13 jährige Eiche; Saat und Pflanzung

Tabelle 25: Mittlere Probebaumparameter $\mathbf{x}$ und Standardabweichungen (sx); Holzerode, Buche und Fichte, 55-65 jährig im Rein- und im Mischbestand

Tabelle 26: Mittlere Probebaumparameter $\mathbf{x}$ und Standardabweichungen (sx); Krebeck, 21-41 jährige Fichte im Reinbestand

Tabelle 27: Mittlere Probebaumparameter $\mathbf{x}$ und Standardabweichungen (sx); Knesebeck, 20-50 jährige Fichte und Kiefer im Reinbestand....

Tabelle 28: Mittlere Probebaumparameter $\mathbf{x}$ und Standardabweichungen (sx); Holzminden, 22 jährige Eiche; Saat und Pflanzung

Tabelle 29: Mittlere Probebaumparameter $\mathbf{x}$ und Standardabweichungen (sx); Kattenbühl, 13 jährige Eiche; Saat und Pflanzung

Tabelle 30: $\quad$ Maximale Durchmesser ${ }_{(\text {WDmax })}$; der Wurzeln der ersten Sektion; Holzerode; 5565 jährige Buche im Rein- und Mischbestand

Tabelle 31: Maximale Durchmesser ${ }_{(\text {WDmax }}$; der Wurzeln der ersten Sektion; Krebeck; 2041 jährige Fichte im Reinbestand.

Tabelle 32: Maximale Durchmesser ${ }_{\text {(wDmax) }}$; der Wurzeln der ersten Sektion Knesebeck; Fichte und Kiefer, 20 - 50 jährig im Reinbestand

Tabelle 33: Maximale Durchmesser (WDmax) $_{\text {; }}$; der Wurzeln der ersten Sektion; Holzminden, 22 jährige Eiche aus Saat und Pflanzung

Tabelle 34: Maximale Durchmesser (WDmax) $_{\text {; }}$; der Wurzeln der ersten Sektion; Kattenbühl, Eiche 13 jährig aus Saat und Pflanzung....

Tabelle 35: Summe der Wurzelquerschnittfläche und Wurzelanzahl pro 22 jährige Eiche, differenziert nach Kraft; Holzminden ( ${ }^{*}$ signifikant unterschiedlich $\quad \mathrm{zu}$ ).

Tabelle 36: Mittlere prozentuale Verteilung der Wurzelquerschnittflächen auf die horizontalen und vertikalen Sektionen differenziert nach Kraft'schen Klassen; Holzminden, 22 jährige Eiche (HQF1+HQF2+HQF3=100\%; VQF1+VQF2+VQF3=100\%)

Tabelle 37: Mittlere prozentuale Verteilung der Wurzelanzahl auf die horizontalen und vertikalen Sektionen differenziert nach Kraft'schen Klassen; Holzminden, 22 jährige Eiche (HAF1+HAF2+HAF3=100 \%; VAF1+VAF2+VAF3=100\%)

Tabelle 38: Mittlere prozentuale Verteilung der Wurzelquerschnittfläche (WQF) auf die horizontalen und vertikalen Sektionen; Holzerode, Krebeck, Knesebeck und Kattenbühl; Fichte, Buche, Kiefer und Eiche. 
Tabelle 39: Mittlere prozentuale Verteilung der Wurzeln auf die horizontalen und vertikalen Sektionen; Holzerode, Krebeck, Knesebeck und Kattenbühl; Fichte, Buche, Kiefer und Eiche.

Tabelle 40: Mittlere Relation der horizontalen zur vertikalen Wurzelquerschnittfläche (WQF); Holzerode, Fichte und Buche im Rein- und Mischbestand

Tabelle 41: Mittlere Relation der Horizontal- zu den Vertikalwurzeln (Anzahl); Holzerode, Fichte und Buche

Tabelle 42: Mittlere Relation der horizontalen zur vertikalen Wurzelquerschnittfläche (WQF); Krebeck, Fichte im Reinbestand

Tabelle 43: Mittlere Relation der Horizontal- zu Vertikalwurzel (Anzahl); Krebeck, Fichte im Reinbestand

Tabelle 44: Mittlere Relation der horizontalen zur vertikalen Wurzelquerschnittfläche (WQF); Knesebeck, Fichte und Kiefer im Reinbestand

Tabelle 45: Mittlere Relation der Horizontal- zu den Vertikalwurzeln (Anzahl); Knesebeck, Fichte und Kiefer

Tabelle 46: Mittlere Relation der horizontalen zur vertikalen Wurzelquerschnittläche (WQF) differenziert nach Kraft'schen Klassen; Holzminden, 22 jährige Eiche

Tabelle 47: Mittlere Relation der Horizontal- zu den Vertikalwurzel (Anzahl); Holzminden, 22 jährige Eiche

Tabelle 48: Mittlere Relation der Horizontal- zu den Vertikalwurzeln (Anzahl) sowie der horizontalen zur vertikalen Wurzelquerschnittfläche (WQF); Kattenbühl, 13 jährige Eiche

Tabelle 49: Mittleres Horizontal-, Vertikal- und Gesamtwurzelvolumen je Baum mit Standardabweichung (sx); Holzerode, Fichte und Buche im Rein- und Mischbestand

Tabelle 50: Mittleres Horizontal-, Vertikal- und Gesamtwurzelvolumen je Baum mit Standardabweichung (sx); Krebeck, Fichte im Reinbestand

Tabelle 51: Mittleres Horizontal-, Vertikal- und Gesamtwurzelvolumen je Baum mit Standardabweichung (sx); Knesebeck; Fichte im Reinbestand....

Tabelle 52: Mittleres Horizontal-, Vertikal- und Gesamtwurzelvolumen mit Standardabweichung (sx); Knesebeck, Kiefer im Reinbestand

Tabelle 53: Mittleres Horizontal-, Vertikal- und Gesamtwurzelvolumen je Baum mit Standardabweichung (sx); Holzminden, Eiche, Kraft'sche Klasse 2

( ${ }^{*}$ signifikant zu)

Tabelle 54: Mittleres Horizontal-, Vertikal- und Gesamtwurzelvolumen je Baum mit Standardabweichung (sx); Holzminden, Eiche, Kraft'sche Klasse 4

( ${ }^{*}$ signifikant zu).

Tabelle 55: Mittleres Horizontal-, Vertikal- und Gesamtwurzelvolumen je Baum mit Standardabweichung(sx); Kattenbühl, Eiche ( ${ }^{*}$ signifikant

$\mathrm{zu})$

Tabelle 56: Mittlere Wurzelanteile (Vol. \%) pro $\mathrm{m}^{3}$ Boden, differenziert nach Kraft'schen Klassen; Holzminden; 22 jährige Eiche

Tabelle 57: Mittlere Wurzelanteile (Vol. \%) pro $\mathrm{m}^{3}$ Boden 
Tabelle 58: Mittlere Anzahl Feinwurzeln und Standardabweichung (sx) pro Einzelwurzel eines Wurzelsystems

Tabelle 59: Funktionen der Beziehungen zwischen HEGYI-Index $(X)$ und Wurzelvolumen (Y)

Tabelle 60: $\quad$ Parameter der linearen $\left[Y=A+B^{*} X\right]$ Beziehungen zwischen Wurzel $(Y)$ - und Stammvolumen $(X)$, getrennt nach Baumart; Fichte, Buche, Eiche. Es werden nur Regressionen mit dem Bestimmtheitsmaß $\left(R^{2}\right) \quad 0,5$ dargestellt

Tabelle 61: Wurzelvolumen bei beispielhaft gewähltem Stammvolumen von $0,06 \mathrm{~m}^{3}$ und $0,25 \mathrm{~m}^{3}$; Krebeck, Fichte 21-, 30- und 41 jährig im Reinbestand (21 jähriger Bestand besitzt nur Bäume mit einem Stammvolumen bis $0,11 \mathrm{~m}^{3}$ )

Tabelle 62: Wurzelvolumen bei beispielhaft gewählten Stammvolumina von 0,06 $\mathrm{m}^{3}$; und 0,3 Knesebeck, 20-, 31- und 50 jährige Fichte

Tabelle 63: Wurzelvolumen bei beispielhaft gewählten Stammvolumina von $0,02 \mathrm{~m}^{3}$ und 0,07 m³ Holzminden, 22 jährige Eiche

Tabelle 64: Beispiel einer Berechnung der Parameter eines Baumes anhand der Ausgleichsfunktionen aus Anhang V, Seiten N, 0; Holzerode, Fichte im Reinund im Mischbestand. Eingangsgröße: BHD $27 \mathrm{~cm}$ (Nadelgewicht war bei der Fichte im Mischbestand nicht verfügbar, so daß an dessen Stelle der Kronenansatz berechnet wird.).

Tabelle 65: Beispiel einer Berechnung von Stamm-, Kronen- und Wurzelparametern anhand der Ausgleichsfunktionen aus Anhang V, P-R; Krebeck, Fichte; Eingangsgrößen: zum einen Fichte, 1. Ertragsklasse, und zum anderen Fichte, BHD $14 \mathrm{~cm}$

Tabelle 66: Beispiel einer Berechnung von Stamm-, Kronen- und Wurzelparametern anhand der Ausgleichsfunktionen aus Anhang V, S-W; Knesebeck, Fichte und Kiefer (20-50 jährig); Eingangsgröße BHD des Grundflächenzentralbaumes

Tabelle 67: Wurzel-, Stamm- und Kronenparameter einer 30 bzw. 31 jährigen Fichte auf Lehm und auf Sand bei einem beispielhaft gewählten BHD von $13 \mathrm{~cm}$ (entspricht etwa dem Grundflächenzentralstamm), Berechnung anhand der Funktionan aus Anhang V, Q und T.

Tabelle 68: Beispiel einer Berechnung der Parameter einer 22 jährigen Eiche; Eingangsgrößen: BHD $7 \mathrm{~cm}$ und $12 \mathrm{~cm}$; Holzminden

Tabelle 69: Beispiel einer Berechnung der Parameter; 13 jährige Eiche; BHD $6 \mathrm{~cm}$ und $8 \mathrm{~cm}$; Kattenbühl

Tabelle 70: Übersicht der in der Literatur beschriebenen Methoden für Wurzeluntersuchungen (POLOMSKI und KUHN 1998) .95

Tabelle 71: Radiengrößen zur Wurzelquerschnitterfassung .97

Tabelle 72: Sproß - und Kronenparameter zweier Fichten mit einem beispielhaften Wurzelvolumen von $0,028 \mathrm{~m}^{3}$ auf Lehm und auf Sand 


\section{Abbildungsverzeichnis}

Abb. 1: $\quad$ Lage der Untersuchungsgebiete (Untersuchungsflächen Nr. 1-19) 4

Abb. 2: $\quad$ Skizze der Untersuchungsgebiete Krebeck, 21-, 30- und 41 jährige Fichte 9

Abb. 3: Schematische Darstellung der Probeflächen Holzminden, 22 jährige Eiche, Pflanzung, Saat und Verpflanzung

Abb. 4: Schematische Darstellung der Probeflächen Kattenbühl, 13jährige Eiche, Saat und Pflanzung.....

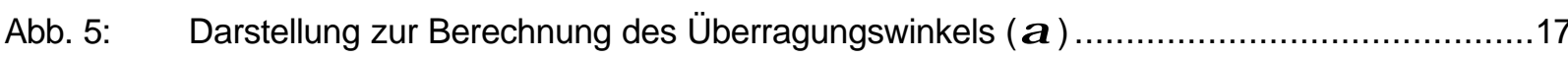

Abb. 6: Wurzelmeßschema

Abb. 7: Erklärende Darstellung der Verhältnisbetrachtung der Horizontal- zu den Vertikalwurzeln in Anzahl (N) und Wurzelquerschnittfläche (WQF $\mathrm{cm}^{2}$ ) in den drei Sektionen. Ausgewogenes Wurzelsystem wird durch eine Relation von 1:1, unausgewogenes durch z.B. 5:1 gekennzeichnet.

Abb. 8: Kegelstumpf

Abb. 9: Schema eines Flußdiagrammes

Abb. 10: Wurzeldurchmesserverteilung (50 mm-Klassen) der Horizontal- und Vertikalwurzeln; Holzerode, 55-68jährig Buche und Fichte im Rein- und Mischbestand

Abb. 11: Wurzeldurchmesserverteilung (50 m-Klassen) der Horizontal- und Vertikalwurzeln; Krebeck, 30- und 41 jährige Fichte im Reinbestand

Abb. 12: Wurzeldurchmesserverteilung (50 mm-Klassen) der Horizontal- und Vertikalwurzeln; Knesebeck, 31 und 50 jährige Fichte im Reinbestand

Abb. 13: Wurzeldurchmesserverteilung (50 mm-Klassen) der Horizontal- und Vertikalwurzeln; Knesebeck, 33- und 47 jährige Kiefer im Reinbestand....

Abb. 14: Wurzeldurchmesserverteilung (20 mm-Klassen) der Horizontal- und Vertikalwurzeln; Holzminden, 22 jährige Eiche aus Saat und Pflanzung

Abb. 15: Wurzeldurchmesserverteilung (20 mm-Klassen) der Horizontal- und Vertikalwurzeln; Kattenbühl, 13 jährige Eiche aus Saat und Pflanzung

Abb. 16: Darstellung der mittleren und maximalen Gesamttiefe und Standardabweichung; Holzerode (Fichte und Buche, im Mittel 60 jährig im Misch- und Reinbestand), Krebeck (Fichte 20-41 jährig, Reinbestand) und Knesebeck (Fichte und Kiefer 20-50 jährig, Reinbestand)

Abb. 17: Darstellung der mittleren und maximalen Gesamttiefe und Standardabweichung der 14- und 22 jährigen Eichen, Holzminden und Kattenbühl

Abb. 18: Mittlere Summe der Wurzelquerschnittfläche und Wurzelanzahl pro Baum und Standardabweichung (1. Sektion); Holzerode, Fichte und Buche ( signifikant $\mathrm{zu})$

Abb. 19: Mittlere Summe der Wurzelquerschnittfläche und Wurzelanzahl pro Baum und Standardabweichung (1. Sektion); Krebeck, Fichte ( signifikant

zu)

Abb. 20: Mittlere Summe der Wurzelquerschnittfläche und Wurzelanzahl pro Baum und Standardabweichung (1. Sektion); Knesebeck, Fichte

Abb. 21: Summe der Wurzelquerschnittfläche und Wurzelanzahl pro Baum und Standardabweichung (1. Sektion); Knesebeck, Kiefer ( signifikant zu).

Abb. 22: Summe der Wurzelquerschnittfläche und Wurzelanzahl pro Baum und Standardabweichung (1. Sektion); Holzminden, 22 jährige Eiche.

Abb. 23: Summe der Wurzelquerschnittfläche und Wurzelanzahl pro Baum und Standardabweichung (1. Sektion); Kattenbühl, 13 jährige Eiche ( signifikant $\mathrm{zu})$ 
Abb. 24: Mittlere Wurzelquerschnittfläche und Wurzelanzahl der horizontalen (HQF), vertikalen (HQF) und Pfahlwurzeln (PQF) eines Wurzelsystems differenziert nach 3 Sektionen, Holzminden, Eiche, Kraft 2. Die große Anzahl der Pfahlwurzeln resultiert aus der Zergliederung der Pfahlwurzel in tieferen Bodenschichten

Abb. 25: Mittleres Wurzelvolumen mit Standardabweichung (sx) differenziert nach sozialer Stellung der Bäume ( $\quad$ signifikant $\quad$ zu); Krebeck und Knesebeck; Fichte und Kiefer

Abb. 26: Oberfläche $\left(\mathrm{dm}^{2}\right)$ der horizontalen und vertikalen Wurzeln, Fichte, Buche und Kiefer; Holzerode, Krebeck und Knesebeck

Abb. 27: Wurzeloberfläche (\%), differenziert nach Wurzelrichtung (horizontal und vertikal); Fichte, Buche und Kiefer; Holzerode, Krebeck und Knesebeck.

Abb. 28: Absolute Verteilung der Wurzeloberfläche $\left(\mathrm{dm}^{2}\right)$ auf horizontale und vertikale Wurzeln; 13- und 22 jährige Eiche, Kattenbühl und Holzminden

Abb. 29: Prozentuale Verteilung der Wurzeloberfläche auf horizontale und vertikale Wurzeln; 13- und 22 jährige Eichen, Kattenbühl und Holzminden

Abb. 30: Geschätzte Anzahl Feinwurzeln pro Wurzelsystem; Holzerode (Fichte und Buche), Krebeck (Fichte) und Knesebeck (Fichte und Kiefer)

Abb. 31: Mittlere geschätzte Anzahl Feinwurzeln pro Baum, differenziert nach Kraft'schen Klassen; Holzminden, Kattenbühl; 13- und 22 jährige Eiche

Abb. 32: Regression zwischen dem HEGYI-Index und dem Wurzelvolumen pro Baum am Beispiel eines 41 jährigen Fichtenbestandes

Abb. 33: Beziehung zwischen Wurzelvolumen $\left(\mathrm{dm}^{3}\right)$ und Stammvolumen $\left(\mathrm{m}^{3}\right)$ auf der Grundlage von allen 262 untersuchten Bäumen (Fichte, Buche, Kiefer und Eiche 14 bis 65 jährig), -------- $95 \%$ Vertrauensintervall (Gerade); $\quad 95 \%$ Vertrauensintervall (Daten)

Abb. 34: Durchschnittliche prozentuale Abweichung der Probebäume mit Standardabweichung (Buche, Fichte und Kiefer) von der in Abb. 33 dargestellten Ausgleichsfunktion. Positive Werte beschreiben die Abweichung der Bäume, die oberhalb der Kurve liegen. Negative stellen die Daten der Bäume dar, die sich unterhalb der Kurve befinden. Die Tabellenwerte geben die Anzahl der Bäume wieder, die ober- oder unterhalb der Kurven liegen

Abb. 35: Durchschnittliche prozentuale Abweichung der Probebäume (Eiche) von der in Abb. 33 dargestellten Ausgleichsfunktion mit Standardabweichung. Positive Werte beschreiben die Abweichung der Bäume, die oberhalb der Kurve liegen. Negative stellen die Daten der Bäume dar, die sich unterhalb der Kurve befinden. Die Tabellenwerte geben die Anzahl der Bäume wieder, die ober- oder unterhalb der Kurven liegen

Abb. 36: Regression zwischen Stamm- und Wurzelvolumen; Holzerode; 55-65jährige Fichte und Buche im Rein- und Mischbestand

Abb. 37: Regression zwischen Stamm- und Wurzelvolumen; Krebeck, 21-, 30- und 41 jährige Fichte. (Die Geraden der 21 jährigen Fichten enden bei einem Stammvolumen von $0,11 \mathrm{~m}^{3}$ und die des 30 jährigen bei $0,27 \mathrm{~m}^{3}$ )....

Abb. 38: Regression zwischen Stamm- und Wurzelvolumen; Knesebeck, 20-, 33- und 47 jährige Fichte. (Die Geraden der 21 jährigen Fichten enden bei einem Stammvolumen von $0,06 \mathrm{~m}^{3}$ und die der 30 jährigen bei $0,32 \mathrm{~m}^{3}$ )

Abb. 39: Regression zwischen Stamm- und Wurzelvolumen; Holzminden, 22 jährige Eiche.

Abb. 40: Regression zwischen Stamm- und Wurzelvolumen; Kattenbühl, 13 jährige Eiche 


\section{Horizontabfolge der verschiedenen Bestände}

Tabelle 1: Bodenbeschreibung der Standorte Holzerode, 55-65jährige Fichte und Buche im Rein- und Mischbestand; Pseudogley-Parabraunerde

\section{Standort Holzerode, Fichte, Buche im Rein- und Mischbestand}

\begin{tabular}{|c|c|c|c|c|c|c|c|c|}
\hline Flächen & Horizont & Tiefe $\mathrm{cm}$ & Bodenart & $\begin{array}{c}\text { Tonanteil } \\
\%\end{array}$ & $\begin{array}{l}\text { Lagerungs- } \\
\text { dichte }\end{array}$ & $\begin{array}{l}\text { Humus- } \\
\text { anteil \% }\end{array}$ & $\begin{array}{l}\text { Skelett- } \\
\text { anteil \% }\end{array}$ & $\mathrm{nWK}$ \\
\hline \multirow[t]{6}{*}{$1-3$} & \multicolumn{2}{|c|}{ Mullartiger Moder } & & & & & & \\
\hline & $\mathrm{Ah} / \mathrm{Al}$ & 10 & $\mathrm{uU}$ & $<5$ & gering & 5 & & 26 \\
\hline & $\mathrm{Bt}$ & 40 & $\mathrm{uU}$ & $<5$ & mittel & & & 75 \\
\hline & Sw & 60 & $u U$ & $<5$ & mittel & & & 50 \\
\hline & $\mathrm{Sd}$ & $>60$ & Ut4 & 25 & mittel & & & 80 \\
\hline & & & & & & & Summe & 231 \\
\hline \multirow[t]{4}{*}{4} & $\mathrm{Ah} / \mathrm{Al}$ & 10 & Uls & 8 & gering & 5 & & 26 \\
\hline & $\mathrm{Bt}$ & 50 & Slu & 12 & mittel & & & 80 \\
\hline & Sw & $>50$ & Uls & 15 & mittel & & & 125 \\
\hline & & & & & & & Summe & 231 \\
\hline
\end{tabular}

Tabelle 2: Bodenbeschreibung der Standorte Krebeck, 21-41 jährige Fichte im Reinbestand; Pseudogley-Parabraunerde

\begin{tabular}{|c|c|c|c|c|c|c|c|c|}
\hline \multicolumn{9}{|c|}{ Standort Krebeck; Fichte 21, 30 und 41 jährig } \\
\hline Flächen & Horizont & Tiefe $\mathrm{cm}$ & Bodenart & $\begin{array}{c}\text { Tonanteil } \\
\%\end{array}$ & $\begin{array}{l}\text { Lagerungs- } \\
\text { dichte }\end{array}$ & $\begin{array}{l}\text { Humus- } \\
\text { anteil \% }\end{array}$ & $\begin{array}{l}\text { Skelett- } \\
\text { anteil \% }\end{array}$ & nWK \\
\hline \multirow[t]{6}{*}{$5-6$} & Moder & & & & & & & \\
\hline & $\mathrm{Ah} / \mathrm{Al}$ & 10 & Us & 5 & gering & 5 & & 26 \\
\hline & $\mathrm{Bt}$ & 50 & Us & 5 & gering & 5 & & 104 \\
\hline & Sw & 70 & lu & 15 & mittel & & & 33 \\
\hline & Sd & $>110$ & It2 & 35 & mittel & & & 60 \\
\hline & & & & & & & Summe & 223 \\
\hline \multirow[t]{6}{*}{7} & Moder & & & & & & & \\
\hline & $\mathrm{Ah} / \mathrm{Al}$ & 10 & Us & 5 & gering & 5 & & 26 \\
\hline & $\mathrm{Bt}$ & 40 & Us & 5 & gering & 5 & & 78 \\
\hline & Sw & 60 & lu & 15 & mittel & & & 33 \\
\hline & Sd & $>100$ & It2 & 35 & mittel & & & 60 \\
\hline & & & & & & & Summe & 197 \\
\hline
\end{tabular}

Tabelle 3: Bodenbeschreibung der Standorte Knesebeck, 20-50 jährige Fichte und Kiefer im Reinbestand; Braunerde-Podsol

\begin{tabular}{|c|c|c|c|c|c|c|c|c|}
\hline \multicolumn{9}{|c|}{ Standort Knesebeck; Fichte und Kiefer, 20-, 30- und 50 jährig } \\
\hline Flächen & Horizont & $\begin{array}{l}\text { Tiefe } \\
\mathrm{cm}\end{array}$ & Bodenart & $\begin{array}{c}\text { Tonanteil } \\
\%\end{array}$ & $\begin{array}{l}\text { Lagerungs- } \\
\text { dichte }\end{array}$ & $\begin{array}{l}\text { Humus- } \\
\text { anteil \% }\end{array}$ & $\begin{array}{l}\text { Skelett- } \\
\text { anteil \% }\end{array}$ & $\begin{array}{c}\mathrm{nW} \\
\mathrm{K}\end{array}$ \\
\hline \multirow[t]{6}{*}{$8-9 ; 11$} & \multicolumn{8}{|c|}{ Rohhumusartiger Mull } \\
\hline & Ahe & 5 & $\mathrm{SI} 2$ & 6 & gering & 0,5 & & 12 \\
\hline & Bhs & 10 & $\mathrm{Sl} 2$ & 6 & gering & 0,5 & & 12 \\
\hline & $\mathrm{Bv}$ & 50 & SI3 & 10 & gering & - & & 92 \\
\hline & \multirow[t]{2}{*}{ Cvll } & $>50$ & LS4 & 18 & dicht & - & & 120 \\
\hline & & & & & & & Summe & 236 \\
\hline \multirow[t]{4}{*}{$10 ; 12-13$} & \multicolumn{8}{|c|}{ Rohhumusartiger Mull } \\
\hline & Ahe & 15 & $\mathrm{mS}$ & - & gering & 0,5 & & 17 \\
\hline & $\mathrm{Bh}$ & 20 & $\mathrm{mS}$ & - & mittel & 4 & & \\
\hline & Bs & 15 & SL2 & 6 & mittel & - & & 12 \\
\hline
\end{tabular}




\begin{tabular}{|ccccccccc|}
\hline \multicolumn{7}{|c|}{ Standort Knesebeck; Fichte und Kiefer, 20-, 30- und 50 jährig } \\
Flächen & Horizont & Tiefe & Bodenart & Tonanteil & Lagerungs- & Humus- & Skelett- & nW \\
& & $\mathrm{cm}$ & & $\%$ & dichte & anteil \% & anteil \% & $\mathrm{K}$ \\
& $\mathrm{Bv}$ & 60 & $\mathrm{SI}$ & 18 & gering & - & & 81 \\
& $\mathrm{Cvll}$ & $>60$ & $\mathrm{mS}$ & - & gering & & & 34 \\
& & & & & & & Summe & $\mathbf{1 4 4}$ \\
\hline
\end{tabular}

Tabelle 4: Bodenbeschreibung der Standorte Holzminden, 22 jährige Eiche, Saat, Pflanzung und Verpflanzung; Pseudogley-Parabraunerde

\begin{tabular}{|c|c|c|c|c|c|c|c|c|}
\hline \multicolumn{9}{|c|}{ Standort Holzminden; Eiche, 22 jährig } \\
\hline Flächen & Horizont & $\begin{array}{l}\text { Tiefe } \\
\mathrm{cm}\end{array}$ & Bodenart & $\begin{array}{c}\text { Tonanteil } \\
\%\end{array}$ & $\begin{array}{l}\text { Lagerungs- } \\
\text { dichte }\end{array}$ & $\begin{array}{l}\text { Humus- } \\
\text { anteil \% }\end{array}$ & $\begin{array}{l}\text { Skelett- } \\
\text { anteil \% }\end{array}$ & nWK \\
\hline \multirow[t]{6}{*}{15} & F- Mull & & & & & & & \\
\hline & $\mathrm{Ah} / \mathrm{Al}$ & 10 & Us & 10 & gering & 5 & 10 & 23 \\
\hline & $\mathrm{Bt}$ & 60 & Us & 10 & gering & 5 & 10 & 117 \\
\hline & Sw & 80 & Us & 10 & mittel & 5 & 10 & 45 \\
\hline & $\mathrm{Cv} / \mathrm{Sd}$ & $>80$ & Lt2 & 50 & dicht & 25 & 50 & 12 \\
\hline & & & & & & & Summe & 197 \\
\hline \multirow[t]{6}{*}{$14 ; 16$} & F-Mull & & & & & & & \\
\hline & $\mathrm{Ah} / \mathrm{Al}$ & 10 & Us & 10 & gering & 5 & 10 & 23 \\
\hline & $\mathrm{Bt}$ & 60 & Us & 10 & gering & 5 & 10 & 117 \\
\hline & Sw & 70 & Us & 10 & mittel & 5 & 10 & 23 \\
\hline & $\mathrm{Cv} / \mathrm{Sd}$ & $>70$ & Lt2 & 50 & dicht & 25 & 50 & 12 \\
\hline & & & & & & & Summe & 175 \\
\hline
\end{tabular}

Tabelle 5: Bodenbeschreibung der Standorte Kattenbühl, 13 jährige Eiche, Pflanzung, Saatl und Saatll; Stagnogley

\begin{tabular}{|c|c|c|c|c|c|c|c|c|}
\hline \multicolumn{9}{|c|}{ Standort Kattenbühl; Eiche, 13 jährig } \\
\hline Flächen & Horizont & $\begin{array}{l}\text { Tiefe } \\
\mathrm{cm}\end{array}$ & Bodenart & $\begin{array}{c}\text { Tonanteil } \\
\%\end{array}$ & $\begin{array}{l}\text { Lagerungs- } \\
\text { dichte }\end{array}$ & $\begin{array}{l}\text { Humus- } \\
\text { anteil \% }\end{array}$ & $\begin{array}{l}\text { Skelett- } \\
\text { anteil\% }\end{array}$ & nWK \\
\hline \multirow[t]{6}{*}{17} & F- Mull & & & & & & & \\
\hline & Ap & 20 & Ls2 & 20 & gering & 5 & & 52 \\
\hline & AhSw & 35 & Ls2 & 20 & mittel & 5 & & 37 \\
\hline & Sw & 50 & Ls2 & 25 & mittel & 5 & & 34 \\
\hline & $\mathrm{Sd}$ & $>50$ & Lt2 & 40 & dicht & & & 12 \\
\hline & & & & & & & Summe & 135 \\
\hline \multirow[t]{6}{*}{18} & F- Mull & & & & & & & \\
\hline & Ap & 10 & Ls2 & 20 & gering & 5 & & 26 \\
\hline & AhSw & 25 & Ls2 & 20 & mittel & 5 & & 37 \\
\hline & Sw & 35 & Ls2 & 25 & mittel & 5 & & 23 \\
\hline & Srd & $>35$ & Lt2 & 40 & dicht & & & 12 \\
\hline & & & & & & & Summe & 98 \\
\hline \multirow[t]{6}{*}{19} & F- Mull & & & & & & & \\
\hline & $A p$ & 10 & Ls2 & 20 & gering & 5 & & 26 \\
\hline & AhSw & 25 & Ls2 & 20 & mittel & 5 & & 37 \\
\hline & Sw & 45 & Ls2 & 25 & mittel & 5 & & 46 \\
\hline & Srd & $>45$ & Lt2 & 40 & dicht & & & 8 \\
\hline & & & & & & & Summe & 117 \\
\hline
\end{tabular}


Tabelle 6: Bodenchemie der Standorte Holzerode, Fichte und Buche im Rein- und Mischbestand (55-65 jährig); Krebeck 21-41 jährig im Reinbestand; Pseudogley-Parabraunerde

\begin{tabular}{|c|c|c|c|c|c|c|c|c|c|c|}
\hline \multicolumn{11}{|c|}{$\begin{array}{l}\text { Standort Holzerode } \\
\end{array}$} \\
\hline \multirow[t]{2}{*}{ Bestand (Nr.) } & \multirow[t]{2}{*}{ Horizont } & \multicolumn{5}{|c|}{ Austauschbare Kationen $(\mathrm{AKE})_{\mu} \mathrm{mol}_{c} / \mathbf{g}$} & \multicolumn{3}{|c|}{ Elemente in Festphase $\mathrm{mg} / \mathrm{kg}$} & \multirow{2}{*}{$\begin{array}{c}\text { pHKC } \\
\text { I }\end{array}$} \\
\hline & & H & K & $\mathrm{Ca}$ & Mg & Al & C & $\mathbf{N}$ & $\mathbf{P}$ & \\
\hline \multirow[t]{4}{*}{ Buche/Buche (1) } & $\mathrm{Ol} / \mathrm{Fh}$ & 0,0 & 14,8 & 167,5 & 33,7 & 6,2 & 256100 & 1310 & 864,5 & 3,9 \\
\hline & $\mathrm{Ah} / \mathrm{Al}$ & 6,1 & 2,6 & 25,7 & 8,5 & 31,1 & 60800 & 2900 & 346,5 & 3,4 \\
\hline & $\mathrm{Bt} / \mathrm{Sw}$ & 0,3 & 1,0 & 2,5 & 1,1 & 31,1 & 8200 & 400 & 548,5 & 3,6 \\
\hline & $\mathrm{Sd}$ & 0,3 & 1,3 & 2,5 & 0,8 & 34,2 & 2800 & 300 & 560,2 & 3,5 \\
\hline \multirow[t]{4}{*}{ Buche/Fichte (2) } & $\mathrm{Ol} / \mathrm{Fh}$ & 0,0 & 7,7 & 329,8 & 137,3 & 0,0 & 159100 & 8100 & 593,8 & 6,3 \\
\hline & $\mathrm{Ah}$ & 0,2 & 2,3 & 58,5 & 31,8 & 3,6 & 49100 & 2500 & 422,7 & 4,3 \\
\hline & $\mathrm{Al}$ & 0,7 & 1,3 & 2,4 & 1,6 & 21,8 & 5900 & 300 & 202,8 & 3,6 \\
\hline & $\mathrm{Bt} / \mathrm{Sw}$ & 1,1 & 3,3 & 19,8 & 5,1 & 46,3 & 3200 & 300 & 298,6 & 3,4 \\
\hline \multirow[t]{3}{*}{ Fichte/Fichte (3) } & $\mathrm{Ah} / \mathrm{Al}$ & 1,4 & 2,0 & 72,1 & 40,8 & 6,7 & 69800 & 3100 & 275,6 & 3,9 \\
\hline & $\mathrm{Bt} / \mathrm{Sw}$ & 0,6 & 0,8 & 2,4 & 2,5 & 32,5 & 8300 & 400 & 135,0 & 6,1 \\
\hline & $\mathrm{Sd}$ & 0,2 & 1,3 & 1,6 & 1,3 & 32,0 & 2000 & 200 & 215,0 & 3,6 \\
\hline \multirow[t]{3}{*}{ Fichte/Buche(4) } & Ah & 0,7 & 1,3 & 2,4 & 1,6 & 21,8 & 5900 & 300 & 202,8 & 3,6 \\
\hline & $\mathrm{Al}$ & 0,7 & 1,3 & 2,4 & 1,6 & 21,8 & 5900 & 300 & 202,8 & 3,6 \\
\hline & $\mathrm{Bt} / \mathrm{Sw}$ & 1,1 & 3,3 & 19,8 & 5,1 & 46,3 & 3200 & 300 & 298,6 & 3,4 \\
\hline
\end{tabular}

\begin{tabular}{|c|c|c|c|c|c|c|c|c|c|c|}
\hline \multicolumn{11}{|c|}{ Standort Krebeck } \\
\hline \multirow[t]{2}{*}{ Bestand (Nr.) } & \multirow[t]{2}{*}{ Horizont } & \multicolumn{5}{|c|}{ Austauschbare Kationen (AKE) $\mu$ molc/g } & \multicolumn{3}{|c|}{ Elemente in Festphase mg/kg } & \multirow[t]{2}{*}{$\mathrm{pHKCl}$} \\
\hline & & $\mathrm{H}$ & $\mathrm{K}$ & $\mathrm{Ca}$ & $\mathrm{Mg}$ & Al & C & $\mathrm{N}$ & $\mathrm{P}$ & \\
\hline \multirow[t]{3}{*}{ Fichte 21 (5) } & $\mathrm{Ol} / \mathrm{Fh}$ & 59,3 & 8,3 & 28,4 & 12,2 & 37,8 & 187400 & 9000 & 673,3 & 2,9 \\
\hline & $\mathrm{Ah} / \mathrm{Al}$ & 28,6 & 3,4 & 9,2 & 4,7 & 60,9 & 133700 & 6000 & 478,0 & 2,9 \\
\hline & $\mathrm{Bt} / \mathrm{Sw}$ & 1,9 & 1,4 & 3,3 & 0,8 & 39,1 & 13300 & 600 & 151,9 & 3,5 \\
\hline \multirow[t]{3}{*}{ Fichte $30(6)$} & $\mathrm{Ol} / \mathrm{Fh}$ & 15,0 & 12,0 & 102,9 & 18,1 & 10,0 & 338800 & 15500 & 884,0 & 3,6 \\
\hline & $\mathrm{Ah} / \mathrm{Al}$ & 3,4 & 1,6 & 11,0 & 2,7 & 27,1 & 99700 & 2600 & 285,3 & 3,5 \\
\hline & $\mathrm{Bt} / \mathrm{Sw}$ & 1,8 & 1,5 & 2,5 & 1,1 & 31,1 & 8400 & 400 & 169,7 & 3,4 \\
\hline \multirow[t]{3}{*}{ Fichte 41 (7) } & $\mathrm{Ol} / \mathrm{Fh}$ & 13,1 & 8,5 & 88,2 & 15,8 & 15,6 & 214000 & 9700 & 701,4 & 3,6 \\
\hline & $\mathrm{Ah} / \mathrm{Al}$ & 9,1 & 1,3 & 7,9 & 1,9 & 44,5 & 31200 & 1500 & 238,3 & 3,1 \\
\hline & $\mathrm{Bt} / \mathrm{Sw}$ & 2,0 & 1,0 & 2,1 & 0,7 & 38,7 & 10100 & 500 & 134,0 & 3,4 \\
\hline
\end{tabular}


Tabelle 7: Bodenchemie der Standorte Krebeck 21-41 jährig im Reinbestand, Pseudogley-Parabraunerde, Knesebeck, Fichte und Kiefer 20-50 jährig, Braunerde-Podsol

\begin{tabular}{|c|c|c|c|c|c|c|c|c|c|c|}
\hline \multicolumn{11}{|c|}{$\begin{array}{l}\text { Standort Knesebeck } \\
\end{array}$} \\
\hline \multirow{2}{*}{ Bestand (Nr.) } & \multirow[t]{2}{*}{ Horizont } & \multicolumn{5}{|c|}{ Austauschbare Kationen (AKE) $\mu$ molc/g } & \multicolumn{3}{|c|}{ Elemente in Festphase $\mathrm{mg} / \mathrm{kg}$} & \multirow[t]{2}{*}{$\mathrm{pH}_{\mathrm{kCl}}$} \\
\hline & & $\mathbf{H}$ & $\mathbf{K}$ & $\mathbf{C a}$ & $\mathbf{M g}$ & Al & C & $\mathbf{N}$ & $\mathbf{P}$ & \\
\hline \multirow[t]{3}{*}{ Fichte 20 (8) } & Ahe & 4,6 & 0,9 & 30,0 & 4,9 & 17,8 & 72900 & 3300 & 165,1 & 3,5 \\
\hline & $\mathrm{Bhs} / \mathrm{Bv}$ & 3,7 & 0,4 & 3,1 & 0,8 & 12,5 & 12300 & 500 & 60,8 & 3,4 \\
\hline & Cvll & 0,0 & 0,2 & 1,1 & 0,3 & 8,0 & 5200 & 200 & 56,1 & 4,3 \\
\hline \multirow[t]{3}{*}{ Fichte 31 (9) } & Ahe & 3,9 & 0,8 & 6,2 & 2,6 & 48,9 & 59700 & 2100 & 177,9 & 3,4 \\
\hline & $\mathrm{Bhs} / \mathrm{Bv}$ & 0,0 & 0,3 & 1,0 & 0,3 & 17,3 & 12300 & 500 & 109,6 & 4,2 \\
\hline & Cvll & 0,0 & 0,2 & 0,6 & 0,2 & 11,1 & 1600 & - & 69,1 & 4,2 \\
\hline \multirow[t]{3}{*}{ Fichte 50 (10) } & Ahe & 40,7 & 2,0 & 3,5 & 1,9 & 32,9 & 128199 & 4700 & 220,3 & 2,7 \\
\hline & $\mathrm{Bh} / \mathrm{Bs} / \mathrm{Bv}$ & 0,0 & 0,2 & 0,7 & 0,3 & 15,6 & 8100 & 300 & 71,3 & 4,0 \\
\hline & Cvll & 0,0 & 0,2 & 0,5 & 0,2 & 10,2 & 4900 & 200 & 71,6 & 4,1 \\
\hline \multirow[t]{3}{*}{ Kiefer 20 (11) } & Ahe & 4,6 & 0,9 & 30,0 & 4,9 & 17,8 & 72900 & 3300 & 165,1 & 3,5 \\
\hline & $\mathrm{Bhs} / \mathrm{Bv}$ & 3,7 & 0,4 & 3,1 & 0,8 & 12,5 & 12300 & 500 & 60,8 & 3,4 \\
\hline & Cvll & 0,0 & 0,2 & 1,1 & 0,3 & 8,0 & 5200 & 200 & 56,1 & 4,3 \\
\hline \multirow[t]{3}{*}{ Kiefer 33 (12) } & $\mathrm{Ah} / \mathrm{Al}$ & 1,1 & 0,6 & 14,2 & 2,8 & 48,9 & 58400 & 2000 & 171,3 & 3,6 \\
\hline & $\mathrm{Bt} / \mathrm{Sw}$ & 0,0 & 0,3 & 0,8 & 0,3 & 18,7 & 14000 & 600 & 102,0 & 4,3 \\
\hline & $\mathrm{Cv} / \mathrm{Sd}$ & 0,0 & 0,3 & 0,8 & 0,2 & 11,6 & 1700 & 0 & 66,8 & 4,3 \\
\hline \multirow[t]{3}{*}{ Kiefer 47 (13) } & Ahe & 6,6 & 0,7 & 34,5 & 9,5 & 27,6 & 61800 & 2200 & 198,5 & 3,4 \\
\hline & $\mathrm{Bh} / \mathrm{Bs} / \mathrm{Bv}$ & 0,0 & 0,3 & 1,6 & 0,5 & 15,6 & 9800 & 400 & 112,6 & 4,2 \\
\hline & Cvll & 0,0 & 0,1 & 0,5 & 0,2 & 3,1 & 1100 & 0 & 80,8 & 4,4 \\
\hline
\end{tabular}


Tabelle 8: Bodenchemie des Standortes Holzminden, Eiche 22 jährig, Saat und Pflanzung, Pseudogley-Parabraunerde

\begin{tabular}{|c|c|c|c|c|c|c|c|c|c|c|}
\hline \multicolumn{11}{|c|}{ Standort Holzminden } \\
\hline \multirow[t]{2}{*}{ Bestand (Nr.) } & \multirow[t]{2}{*}{ Horizont } & \multicolumn{5}{|c|}{ Austauschbare Kationen $(\mathrm{AKE})_{\mu} \mathrm{mol}_{c} / \mathrm{g}$} & \multicolumn{3}{|c|}{ Elemente in Festphase $\mathrm{mg} / \mathrm{kg}$} & \multirow[t]{2}{*}{$\mathrm{pH}_{\mathrm{kCl}}$} \\
\hline & & $\mathbf{H}$ & $\mathbf{K}$ & $\mathbf{C a}$ & Mg & Al & C & $\mathbf{N}$ & $\mathbf{P}$ & \\
\hline \multirow[t]{4}{*}{ Ei Pflanz 24 (14) } & $\mathrm{Ah} / \mathrm{Al}$ & 2,2 & 4,5 & 22,8 & 3,9 & 3,0 & 30100 & 2000 & 424,2 & 3,9 \\
\hline & $\mathrm{Bt}$ & 0,7 & 2,9 & 24,8 & 2,3 & 29,4 & 20900 & 1200 & 413,4 & 4,0 \\
\hline & Sw & 0,0 & 1,7 & 39,1 & 1,6 & 22,7 & 4600 & 400 & 145,2 & 4,0 \\
\hline & CvSd & 0,0 & 1,0 & 34,5 & 1,9 & 20,5 & 2200 & 300 & 117,5 & 4,0 \\
\hline \multirow[t]{3}{*}{ Ei Saat 22 (15) } & $\mathrm{Ah} / \mathrm{Al}$ & 2,0 & 6,5 & 39,0 & 7,0 & 18,7 & 31300 & 1900 & 511,8 & 4,2 \\
\hline & $\mathrm{Bt} / \mathrm{Sw}$ & 1,2 & 4,9 & 20,5 & 3,5 & 22,2 & 19899 & 1600 & 978,7 & 4,0 \\
\hline & $\mathrm{Sw} / \mathrm{Cv}$ & 0,0 & 3,8 & 9,1 & 0,8 & 27,1 & 4000 & 400 & 281,2 & 3,7 \\
\hline \multirow[t]{3}{*}{ Ei Verpfl 22 (16) } & $\mathrm{Ah} / \mathrm{Al}$ & 0,0 & 7,9 & 86,7 & 7,5 & 0,0 & 35500 & 2300 & -- & 6,0 \\
\hline & $\mathrm{Bt} / \mathrm{Sw}$ & 0,0 & 6,6 & 87,2 & 6,2 & 0,0 & 16600 & 1100 & 436,5 & 5,9 \\
\hline & CvSd & 0,0 & 3,6 & 78,2 & 9,6 & 4,0 & 2000 & 300 & 223,3 & 5,0 \\
\hline
\end{tabular}


Tabelle 9: Bodenchemie der Standorte Kattenbühl; Eiche 13 jährig, Saat und Pflanzung, Pseudogley-Parabraunerde

\begin{tabular}{|c|c|c|c|c|c|c|c|c|c|c|}
\hline \multicolumn{11}{|c|}{ Standort Kattenbühl } \\
\hline \multirow[t]{2}{*}{ Bestand (Nr.) } & \multirow[t]{2}{*}{ Horizont } & \multicolumn{5}{|c|}{ Austauschbare Kationen $(\mathrm{AKE})_{\mu} \mathrm{mol}_{c} / \mathrm{g}$} & \multicolumn{3}{|c|}{ Elemente in Festphase $\mathrm{mg} / \mathrm{kg}$} & \multirow[t]{2}{*}{$\mathrm{pH}_{\mathrm{kCl}}$} \\
\hline & & $\mathbf{H}$ & $\mathbf{K}$ & $\mathrm{Ca}$ & Mg & Al & C & $\mathbf{N}$ & $\mathbf{P}$ & \\
\hline \multirow[t]{3}{*}{ Ei Pflanz 14 (17) } & Ap & 3,8 & 1,0 & 29,7 & 15,1 & 62,7 & 100500 & 4200 & 303,1 & 3,7 \\
\hline & $\mathrm{Ah} / \mathrm{Sw}$ & 5,2 & 1,8 & 16,2 & 5,3 & 104,1 & 100700 & 3900 & 397,5 & 3,5 \\
\hline & Sd & 0,6 & 0,3 & 5,0 & 1,3 & 35,6 & 10100 & 500 & 117,2 & 3,7 \\
\hline \multirow[t]{3}{*}{ Ei Saatl 12 (18) } & Ap & 2,6 & 2,5 & 62,2 & 9,6 & 39,6 & 187700 & 7900 & 324,0 & 4,0 \\
\hline & $\mathrm{Ah} / \mathrm{Sw}$ & 7,9 & 3,5 & 59,2 & 3,8 & 100,1 & 307600 & 12600 & 529,2 & 3,8 \\
\hline & Sw & 0,0 & 0,4 & 9,6 & 1,2 & 47,6 & 27400 & 900 & 175,6 & 3,7 \\
\hline \multirow[t]{3}{*}{ Ei Saat II 12 (19) } & $A p$ & 4,5 & 2,8 & 48,2 & 7,2 & 72,9 & 166700 & 7700 & 423,1 & 4,1 \\
\hline & $\mathrm{Ah} / \mathrm{Sw}$ & 6,4 & 2,0 & 17,0 & 1,3 & 129,0 & 156200 & 7300 & 465,4 & 3,6 \\
\hline & Srd & 0,0 & 0,5 & 7,1 & 1,5 & 57,8 & 30700 & 1100 & 167,6 & 3,9 \\
\hline
\end{tabular}




\section{Holzerode}

Buche: $\mathbf{N}=10$

Fichte: $\mathrm{N}=10$

Buche / Fichte. $\mathrm{N}=12$

Fichte / Buche: $\mathbf{N}=11$

\section{Krebeck}

Fichte 21 jährig: $\mathbf{N}=15$

Fichte 30 jährig: $\mathbf{N}=15$ 


\section{Knesebeck}

Fichte 20 jährig: $\mathrm{N}=11$

Kiefer 20 jährig: $N=11$

Fichte 31 jährig: $\mathbf{N}=16$

Kiefer 33 jährig: $\mathbf{N}=16$

Fichte 50 jährig: $\mathbf{N}=15$

Kiefer 47 jährig: $\mathbf{N}=15$ 
Holzminden

Eiche Pflanzung: $N=14$

Eiche Saat: $\mathbf{N}=14$

Eiche Verpflanzung: $N=6$
Kattenbühl

Eiche Pflanzung: $\mathbf{N}=16$

Eiche Saat: $\mathbf{N}=15$

Eiche Saat II: $N=15$ 


\section{Tabelle 10: Formelübersicht}

Ausformungskoeffizient eines

Wurzelsystems (Anzahl)

\section{Ausformungskoeffizient eines \\ Wurzelsystems (WQF)}

Feinwurzeln pro Einzelwurzel

Feinwurzeln pro Wurzelsystem

Berechnugn der Anzahl der Konkurrenten eines Probebaumes zur Anwendung des Hegyi Indexes (1974)
$\frac{H N_{i}}{V N_{i}}$

$\mathrm{HN}_{\mathrm{i}}=$ horizontale Wurzelanzahl der i'ten Sektion

$\mathrm{VN}_{\mathrm{i}}=$ vertikale Wurzelanzahl der i'ten Sektion

$W Q F=\frac{H Q F_{i}}{V Q F_{i}}$

$\mathrm{HQF}_{\mathrm{i}}=$ horizontale Querschnittsfläche der i'ten Sektion

$\mathrm{VQF}_{\mathrm{i}}=$ vertikale Querschnittsfläche der i'ten Sektion

$\frac{F W}{W A_{i}}$

$\sum_{i=1}^{N} \frac{F W}{W A_{i}}$

mit $\mathrm{N}=\mathrm{N}_{\mathrm{WA}}$

$D_{t}<\frac{B H D_{p}+B H D_{n}}{8}$

$\mathrm{D}_{\mathrm{t}}=$ Abstand des Probebaumes zum Nachbarn

$\mathrm{BHD}_{\mathrm{p}}=\mathrm{BHD}_{\mathrm{Nes}}$ Probebaumes $_{\mathrm{p}}[\mathrm{cm}]$ BHD $_{n}=$ BHD des Nachbarbaumes $_{n}[\mathrm{~cm}]$

$\mathrm{C}_{\mathrm{li}}=\sum_{j=1}^{n} \frac{\left(B H D_{j} / B H D_{i}\right)}{D_{i j}}$

$\mathrm{BHD}_{\mathrm{j}}=$ BHD des Probebaumes i $[\mathrm{cm}]$

$\mathrm{BHD}_{\mathrm{i}}=\mathrm{BHD}$ des Konkurrenzbaumes $\mathrm{j}[\mathrm{cm}]$

$\mathrm{D}=$ Abstand des Probebaumes zum Nachbarn j [m]

$\mathrm{N}=$ Anzahl der Konkurrenzbäume 


\section{horizontale Wurzelquerschnittfläche $\left(\mathrm{HQF}_{\mathrm{i}}\right)$ der durchschnittlichen Einzelwurzel}

$H Q F_{i}=\frac{\sum_{j=1}^{n} H Q F_{j}}{n}$

$\mathrm{HQF}_{\mathrm{i}}=$ horizontale WQF der EW

$\mathrm{n}=$ Anzahl der horizontalen Einzelwurzeln pro Wurzelsystem

horizontale Wurzelquerschnittfläche eines Baumes $\left(\mathrm{HQF}_{\mathrm{i}}\right)$

$$
H Q F_{i}=\sum_{j=1}^{n} H Q F_{j}
$$

horizontaler Prozentanteil (WQF) der 1.- 3.

$H Q F_{j}[\%]=100 * \frac{H Q F_{i}}{\sum_{i=1}^{3} H Q F_{i}}$

$\mathrm{HQF}_{\mathrm{i}}=$ horizontale Querschnittsfläche der i'ten Sektion

Kronenschirmfläche

$\mathrm{Kf}=\partial^{*} r^{2}$

$\mathrm{K}_{\mathrm{f}}=$ Kronenschirmfläche

$\mathrm{d}=$ durchschnittlicher Kronendurchmesser

$\mathrm{WD}_{\max }$

maximale Wurzeldurchmesser

maximale Wurzeltiefe

mittlere Wurzeltiefe

Stammvolumen $\left(\mathrm{V}_{\mathrm{st}}\right)$
$\mathrm{WT}=$ Wurzeltiefe

$\mathrm{N}=$ Anzahl der Wurzeltiefen

$$
\begin{aligned}
& V_{s t}=\sum_{i=1}^{n}\left(G_{m} * l_{i}\right) \\
& \mathrm{G}_{\mathrm{m}}=\text { Mittengrundfläche des zu } \\
& \quad \text { berechnenden Körpers } \\
& \mathrm{l}_{\mathrm{i}}=\text { Länge des zu berechnenden Körpers }
\end{aligned}
$$




\section{Überragungswinkel}

vertikale Wurzelquerschnittfläche $\left(V F_{i}\right)$ der durchschnittlichen Einzelwurzel

vertikale Wurzelquerschnittfläche des Wurzelsystems $\left(\mathrm{VQF}_{\mathrm{i}}\right)$ (1., 2., oder 3. Sektion)

vertikaler Prozentanteil $\left(W_{\mathrm{W}}\right)$ der 1.-3. Sektion an der gesamten $\mathrm{WQF}_{\mathrm{j}}(1$. Sektion +2 . Sektion + 3.Sektion)

Wurzeloberfläche $\left(W_{m}\right)$

Wurzelquerschnittfläche der Einzelwurzel $\left(W_{\mathrm{EW}}\right)$

Wurzelvolumen $\left(\mathrm{V}_{\mathrm{w}}\right)$ $\tan \alpha=\frac{H_{K}-H_{z}}{D}$

$\mathrm{D}=$ Baumabstand

$\alpha=$ Überragungswinkel

$\mathrm{H}_{\mathrm{K}}=$ Höhe des Konkurrenten

$\mathrm{H}_{\mathrm{Z}}=$ Höhe des Zentralbaumes

$V Q F_{i}=\frac{\sum_{j=1}^{n} V Q F_{j}}{n}$

$\mathrm{VQF}_{\mathrm{i}}=$ vertikale WQF der EW

$\mathrm{n}=$ Anzahl der vertikalen Einzelwurzeln pro Wurzelsystem

$V Q F_{i}=\sum_{j=1}^{n} V Q F_{j}$

$\mathrm{VQF}_{\mathrm{i}}=$ Summe der vertikalen Wurzelquerschnittflächen

$V Q F_{j}[\%]=100 * \frac{V Q F_{i}}{\sum_{i=1}^{3} V Q F_{i}}$

$\mathrm{VQF}_{\mathrm{i}}=$ horizontale Querschnittsfläche der i'ten Sektion

$W_{m}=\pi^{*} s\left(R_{0}+r_{u}\right)+\pi+r_{u^{2}}+R^{*} d_{o}^{2}$

$W_{m}=$ Wurzeloberfläche

$\mathrm{S}=$ Wurzelmantellinie

$\mathrm{R}_{\mathrm{o}}=$ oberer Durchmesser

$r_{\mathrm{u}}=$ unterer Durchmesser

$\mathrm{WQF}_{\mathrm{EW}}=\left(\frac{d_{1}}{2} * \frac{d_{2}}{2}\right) * \pi$

$\mathrm{d}_{1}=$ Durchmesser 1. Kluppung

$\mathrm{d}_{2}=$ Durchmesser 2. Kluppung

$v_{W}=\frac{l *\left(G_{u}+4 * G_{m}+G_{o}\right)}{6}$

1 = Länge des Wurzelabschnittes

$\mathrm{g}_{\mathrm{u}}=$ untere Grundfläche

$\mathrm{g}_{\mathrm{m}}=$ mittlere Grundfläche

$\mathrm{g}_{\mathrm{o}}=$ obere Grundfläche 
Tabelle 11: Ertragskundliche Daten, differenziert nach Kraft'schen Klassen; Mittelwerte mit Standardabweichung(Sx); Holzerode

\begin{tabular}{|c|c|c|c|c|c|}
\hline & & Buche & Buche / Fichte & Fichte & Fichte / Buche \\
\hline 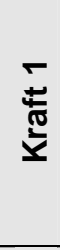 & $\begin{array}{l}\text { BHD }(\mathrm{cm}) \\
\text { Höhe }(\mathrm{m}) \\
\text { H/D-Wert } \\
\text { Stammvolumen }\left(\mathrm{m}^{3}\right) \\
\text { Probebaumanzahl }(\mathrm{n}) \\
\text { Alter (Jahre) }\end{array}$ & & $\begin{array}{r}34,5 \\
21 \\
60,9 \\
0,1 \\
1 \\
60 \\
\end{array}$ & & $\begin{array}{r}34,3(5,1) \\
27,8(0,1) \\
81,7(12,4) \\
1,1(0,1) \\
2 \\
71,5(0,7) \\
\end{array}$ \\
\hline 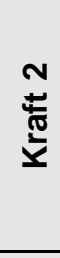 & $\begin{array}{l}\text { BHD }(\mathrm{cm}) \\
\text { Höhe }(\mathrm{m}) \\
\text { H/D-Wert } \\
\text { Stammvolumen }\left(\mathrm{m}^{3}\right) \\
\text { Probebaumanzahl }(\mathrm{n}) \\
\text { Alter (Jahre) } \\
\end{array}$ & $\begin{array}{r}22,3(2,5) \\
25,6(0,8) \\
116,5(16,0) \\
0,5(0,1) \\
4 \\
61,3(3,3) \\
\end{array}$ & $\begin{array}{r}21,5(1,4) \\
23,5(0,2) \\
109,5(7,2) \\
0,3(0,2) \\
2 \\
57,5(3,5) \\
\end{array}$ & $\begin{array}{r}27,8(2,9) \\
28,0(2,7) \\
100,8(2,2) \\
10 \\
55,4(1,2) \\
\end{array}$ & $\begin{array}{r}32,1(1,9) \\
25,2(0,5) \\
78,7(4,2) \\
0,9(0,1) \\
4 \\
65,8(1,7) \\
\end{array}$ \\
\hline 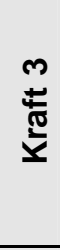 & $\begin{array}{l}\text { BHD }(\mathrm{cm}) \\
\text { Höhe }(\mathrm{m}) \\
\text { H/D-Wert } \\
\text { Stammvolumen }\left(\mathrm{m}^{3}\right) \\
\text { Probebaumanzahl }(\mathrm{n}) \\
\text { Alter (Jahre) }\end{array}$ & $\begin{array}{r}20,6(2,7) \\
24,3(1,5) \\
119,4(11,4) \\
0,4(0,1) \\
6 \\
58,4(5,5) \\
\end{array}$ & $\begin{array}{r}20,3(3,2) \\
21,9(2,9) \\
109,1(16,1) \\
0,4(0,1) \\
9 \\
61,3(7,2) \\
\end{array}$ & & $\begin{array}{r}25,0(5,3) \\
23,5(1,3) \\
96,3(16,6) \\
0,6(0,2) \\
3 \\
63(9,9) \\
\end{array}$ \\
\hline 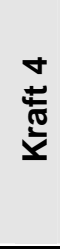 & $\begin{array}{l}\text { BHD }(\mathrm{cm}) \\
\text { Höhe }(\mathrm{m}) \\
\text { H/D-Wert } \\
\text { Stammvolumen }\left(\mathrm{m}^{3}\right) \\
\text { Probebaumanzahl }(\mathrm{n}) \\
\text { Alter (Jahre) }\end{array}$ & & & & $\begin{array}{r}17,5(2,8) \\
19,3(2,2) \\
110,9(5,2) \\
0,2(0,1) \\
2 \\
61(9,9) \\
\end{array}$ \\
\hline
\end{tabular}

Tabelle 12: Kronenparameter, differenziert nach Kraft'schen Klassen; Mittelwerte mit Standardabweichung(Sx); Holzerode

\begin{tabular}{|c|c|c|c|c|c|}
\hline & Kraft 1 & Buche & Buche / Fichte & Fichte & Fichte / Buche \\
\hline$\frac{\overline{ \pm}}{\frac{\pi}{\pi}}$ & $\begin{array}{l}\text { TG Krone }(\mathrm{kg}) \\
\text { Kronenansatz }(\mathrm{m}) \\
\text { Kronenlänge }(\mathrm{m}) \\
\text { Kronenprozent }(\%) \\
\text { Kronenfläche }\left(\mathrm{m}^{2}\right)\end{array}$ & & $\begin{array}{r}97,4 \\
7,7 \\
13,3 \\
63,3 \\
44,9\end{array}$ & & $\begin{array}{r}57,1(10,0) \\
18,5(1,0) \\
9,2(0,9) \\
33,3(3,3) \\
11,9(9,7)\end{array}$ \\
\hline 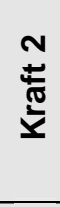 & $\begin{array}{l}\text { TG Krone }(\mathrm{kg}) \\
\text { Kronenansatz }(\mathrm{m}) \\
\text { Kronenlänge }(\mathrm{m}) \\
\text { Kronenprozent }(\%) \\
\text { Kronenfläche }\left(\mathrm{m}^{2}\right)\end{array}$ & $\begin{array}{r}17,3(7,8) \\
16,8(3,3) \\
8,8(2,3) \\
34,5(11,9) \\
15,0(7,4) \\
\end{array}$ & $\begin{array}{r}53,9(18,4) \\
8,6(2,7) \\
14,9(2,7) \\
63,4(11,4) \\
45,5(7,8) \\
\end{array}$ & $\begin{array}{r}56,6(19,7) \\
14,5(1,3) \\
13,5(3,0) \\
47,8(6,2) \\
9,4(2,2) \\
\end{array}$ & $\begin{array}{r}49,0(17,5) \\
17,1(1,44) \\
8,0(1,7) \\
31,9(6,3) \\
8,7(1,0) \\
\end{array}$ \\
\hline 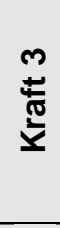 & $\begin{array}{l}\text { TG Krone }(\mathrm{kg}) \\
\text { Kronenansatz }(\mathrm{m}) \\
\text { Kronenlänge }(\mathrm{m}) \\
\text { Kronenprozent }(\%) \\
\text { Kronenfläche }\left(\mathrm{m}^{2}\right) \\
\end{array}$ & $\begin{array}{l}19,2(7,1) \\
13,9(2,3) \\
10,4(2,2) \\
42,8(8,5) \\
17,4(7,3) \\
\end{array}$ & $\begin{array}{r}29,3(10,6) \\
8,9(4,1) \\
12,9(2,6) \\
60,2(15,3) \\
43,2(21,7) \\
\end{array}$ & & $\begin{array}{r}44,2(14,0) \\
15,4(1,03) \\
8,1(0,5) \\
34,4(1,7) \\
12,4(1,2) \\
\end{array}$ \\
\hline$\sum_{\frac{\pi}{\grave{L}}}^{+}$ & $\begin{array}{l}\text { TG Krone }(\mathrm{kg}) \\
\text { Kronenansatz }(\mathrm{m}) \\
\text { Kronenlänge }(\mathrm{m}) \\
\text { Kronenprozent }(\%) \\
\text { Kronenfläche }\left(\mathrm{m}^{2}\right)\end{array}$ & & & & $\begin{array}{r}15,77(4,94) \\
12,98(4,49) \\
6,35(2,3) \\
33,76(15,6) \\
13,41(2,6)\end{array}$ \\
\hline
\end{tabular}




\section{Holzerode, Fichte im Reinbestand}
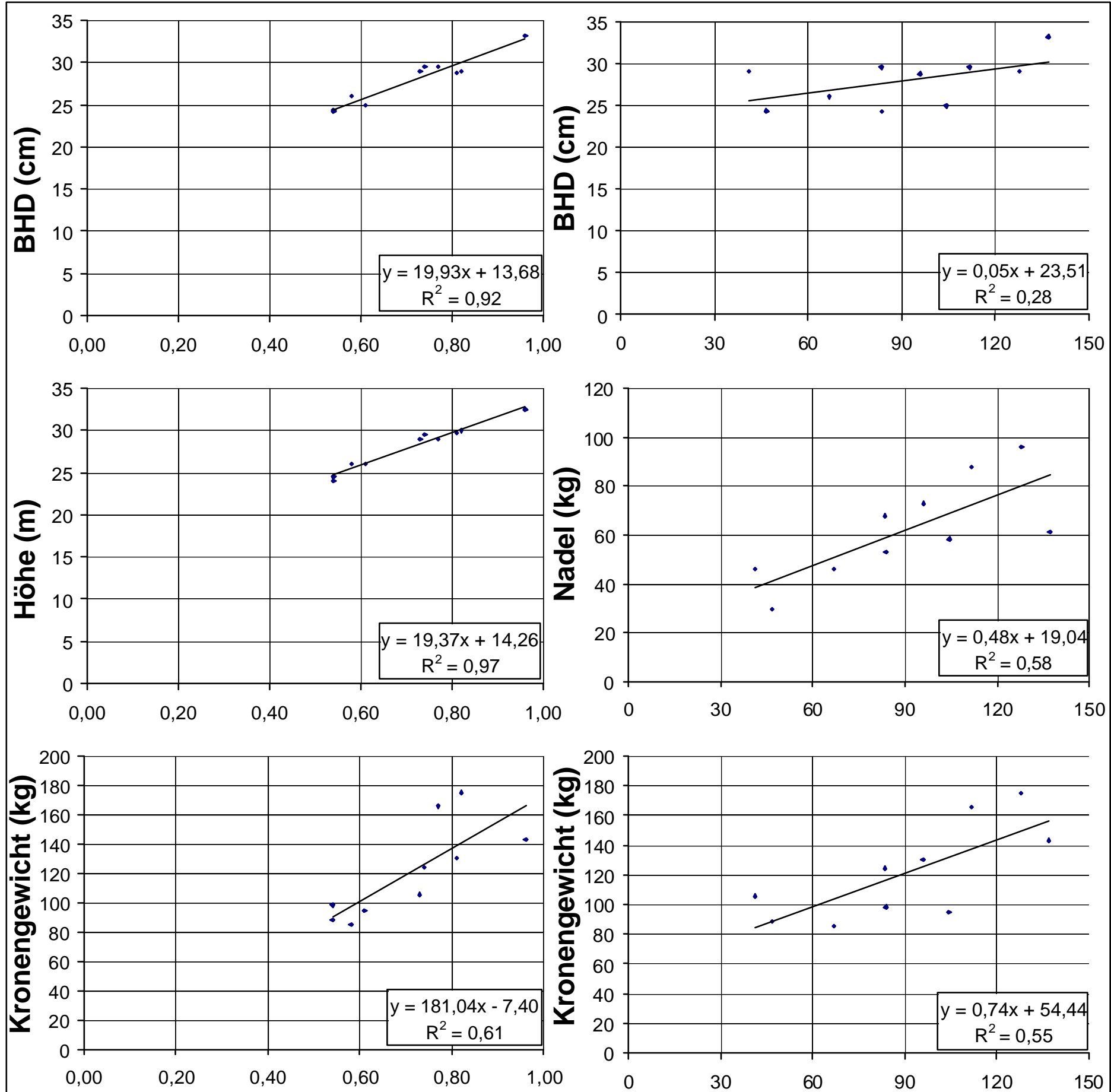

Stammvolumen $\left(\mathrm{m}^{3}\right)$

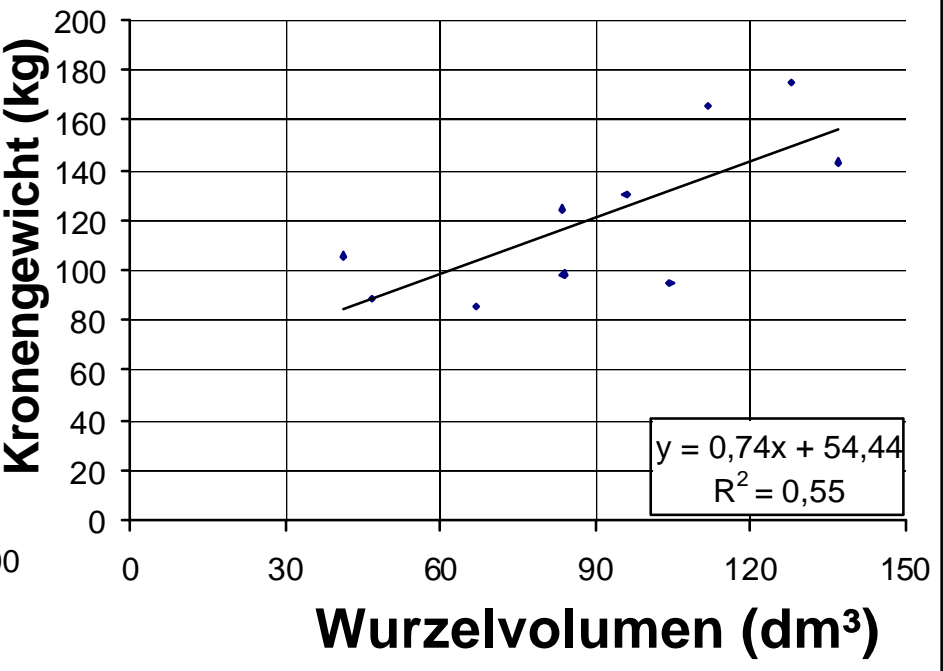

Diagramm 1: Beziehungen zwischen Stamm-, Kronen- und Wurzelparametern; Holzerode, Fichte im Reinbestand (durchschnittlich 55 jährig). Kronenansatz und -Gewicht (im Frischezustand). 


\section{Holzerode, Fichte im Mischbestand}
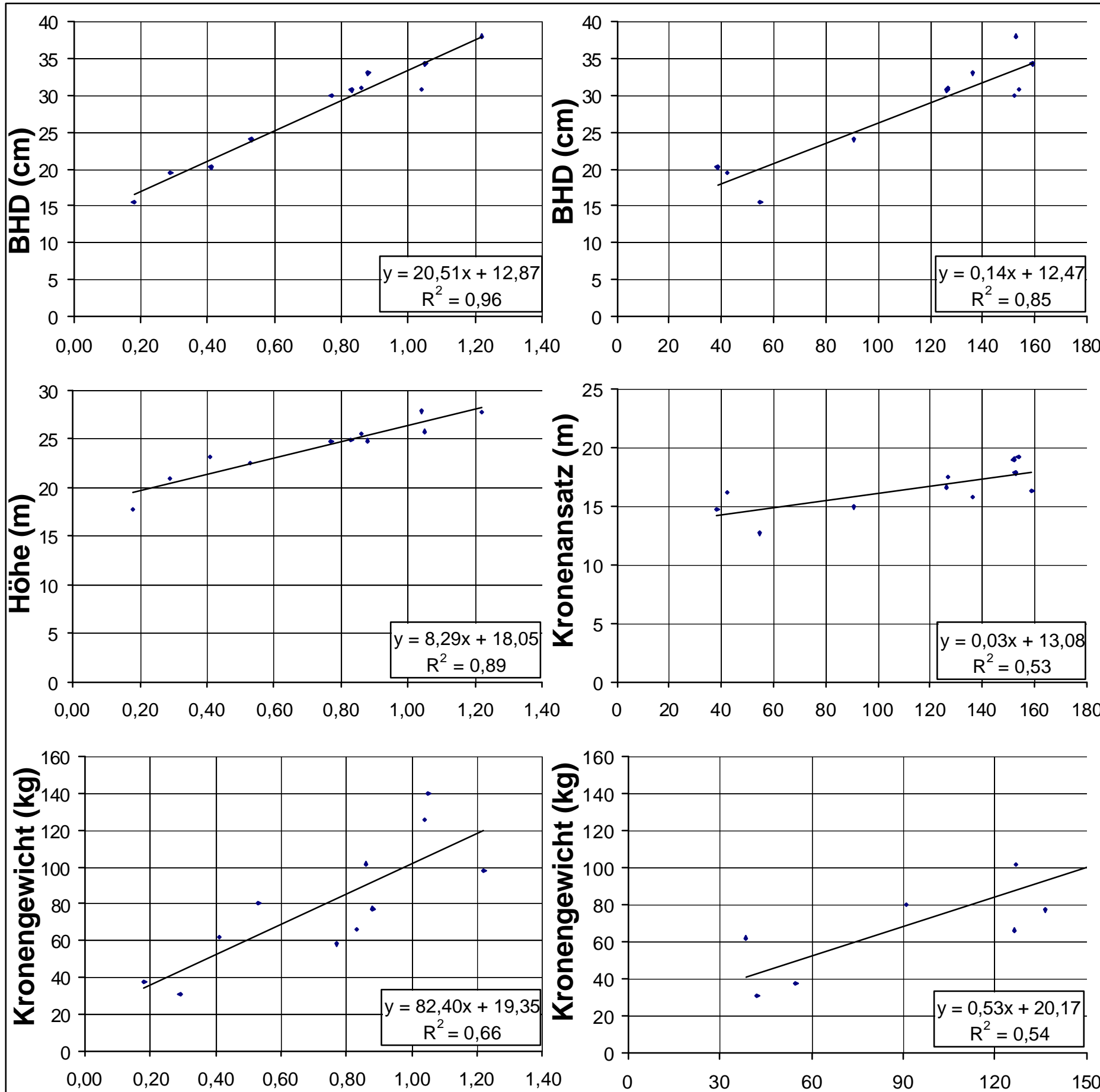

Stammvolumen $\left(\mathrm{m}^{3}\right)$

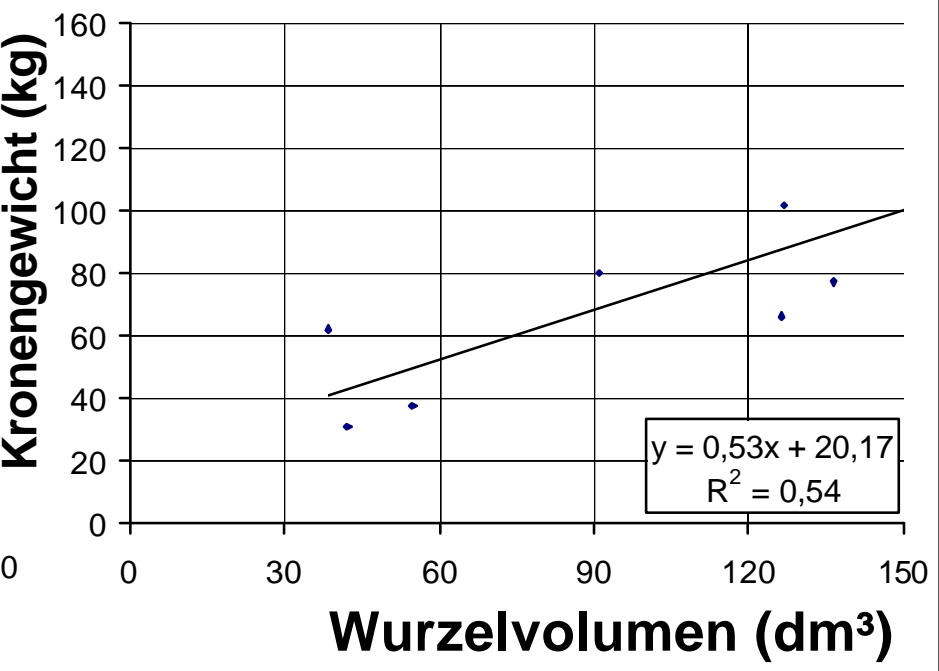

Diagramm 2: Beziehungen zwischen Stamm-, Kronen- und Wurzelparametern; Holzerode, Fichte im Mischbestand (durchschnittlich 65 jährig). Nadel/ Blatt- und Kronengewicht (im Frischezustand). 


\section{Krebeck, Fichte 21 jährig}
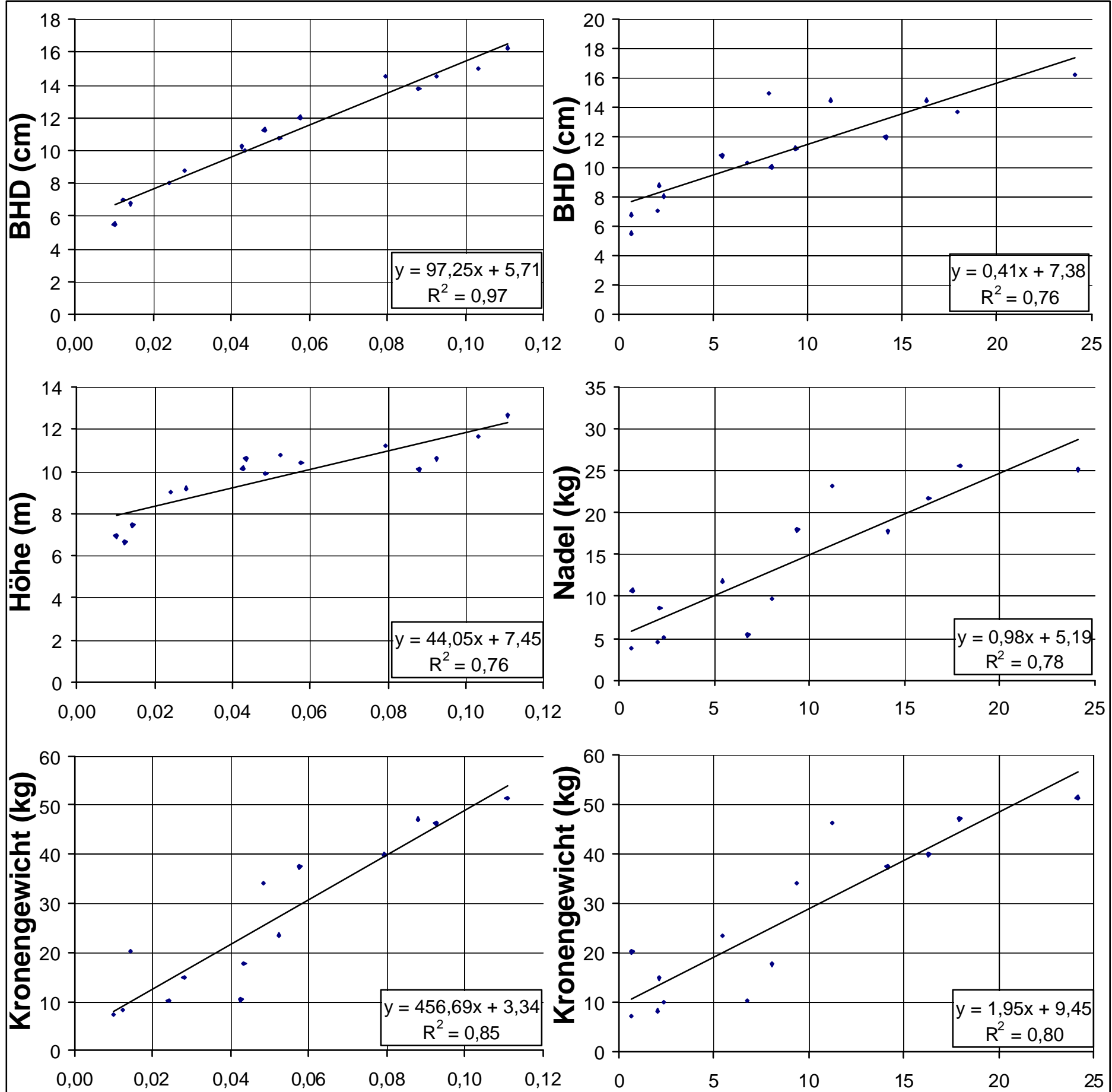

Stammvolumen $\left(\mathrm{m}^{3}\right)$

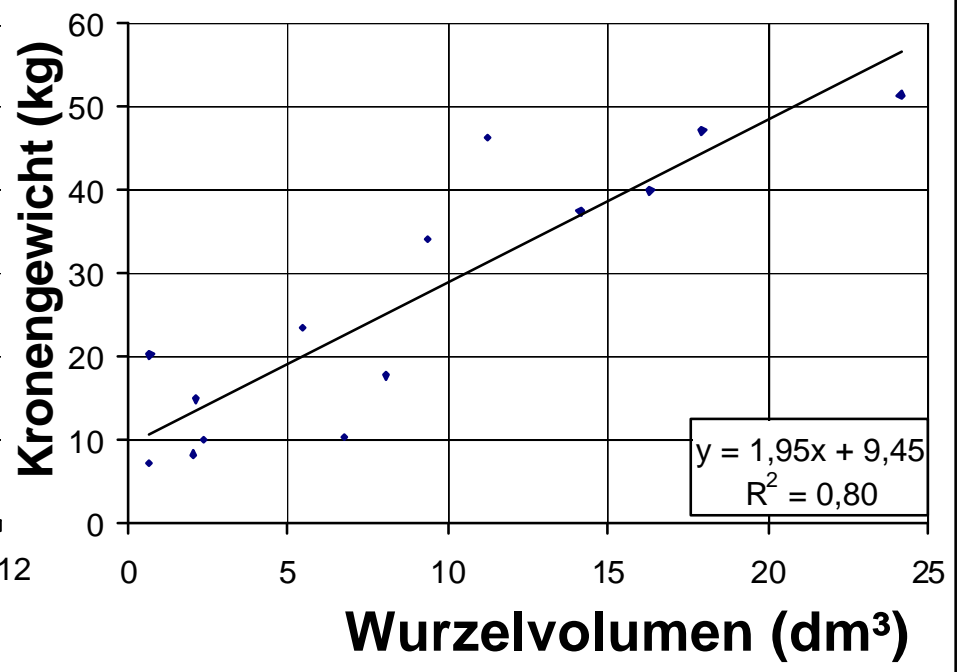

Diagramm 3: Beziehungen zwischen Stamm-, Kronen- und Wurzelparametern; Krebeck, Fichte 21 jährig, Nadel / Blatt- und Kronengewicht (im Frischezustand). 


\section{Krebeck, Fichte 30 jährig}
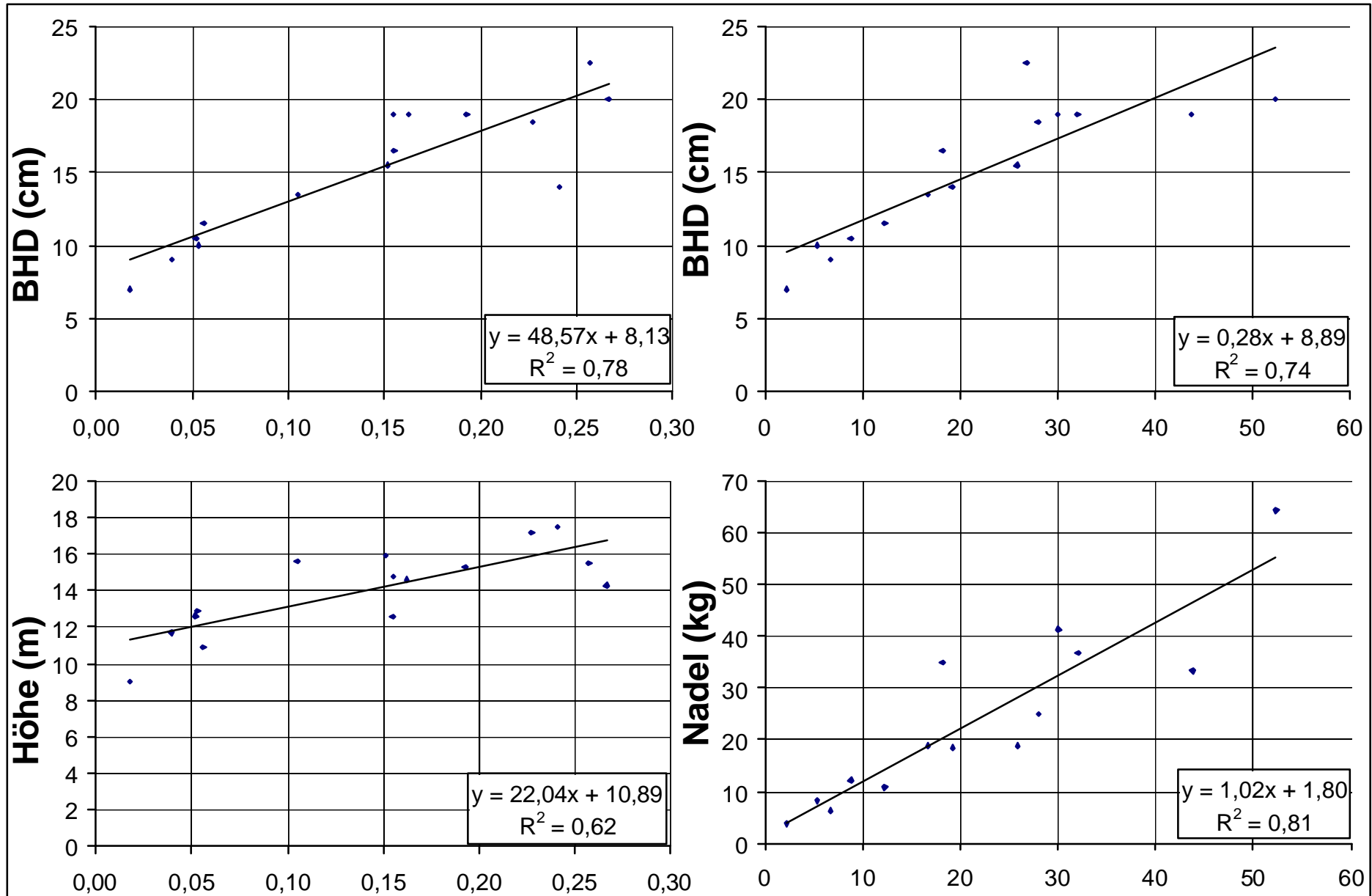

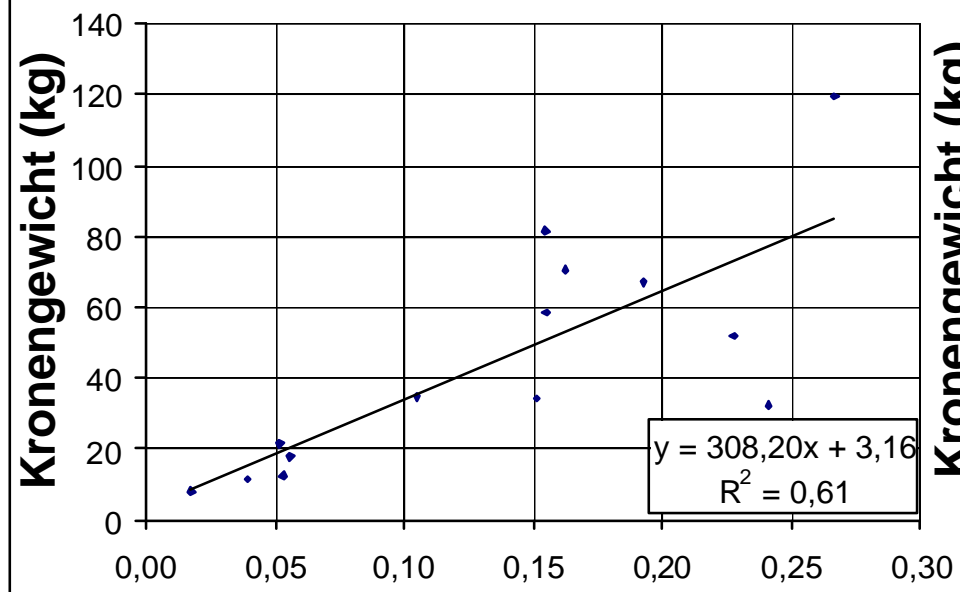

Stammvolumen $\left(\mathrm{m}^{3}\right)$

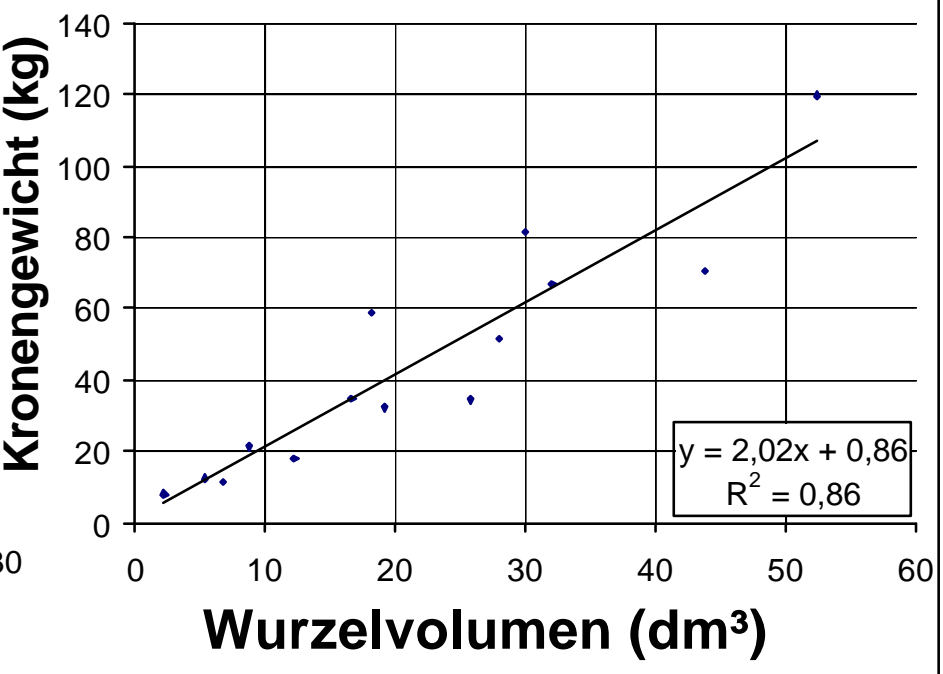

Diagramm 4: Beziehungen zwischen Stamm-, Kronen- und Wurzelparametern; Krebeck, Fichte 30 jährig, Nadel / Blatt- und Kronengewicht (im Frischezustand). 


\section{Krebeck, Fichte 41 jährig}
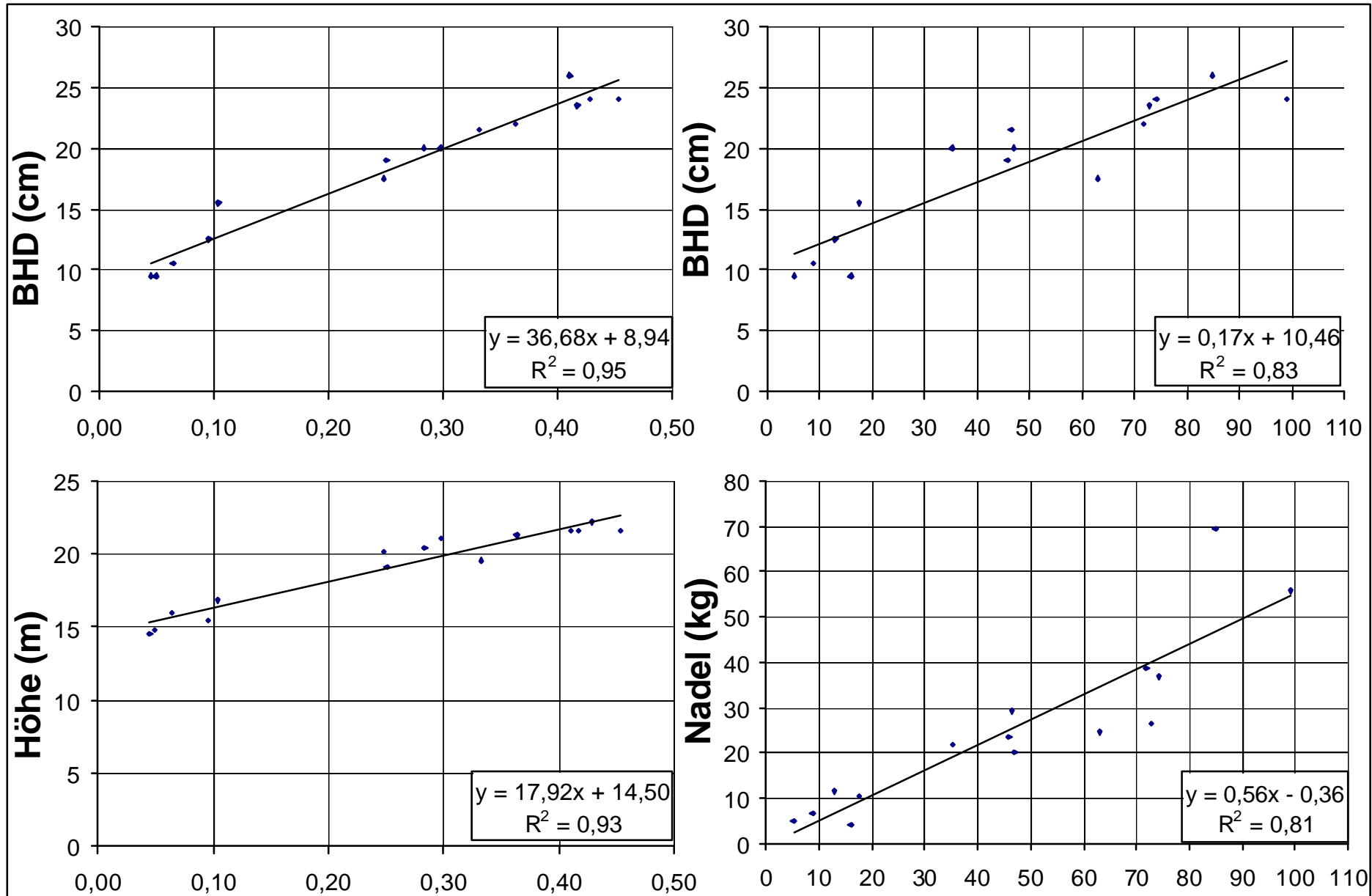

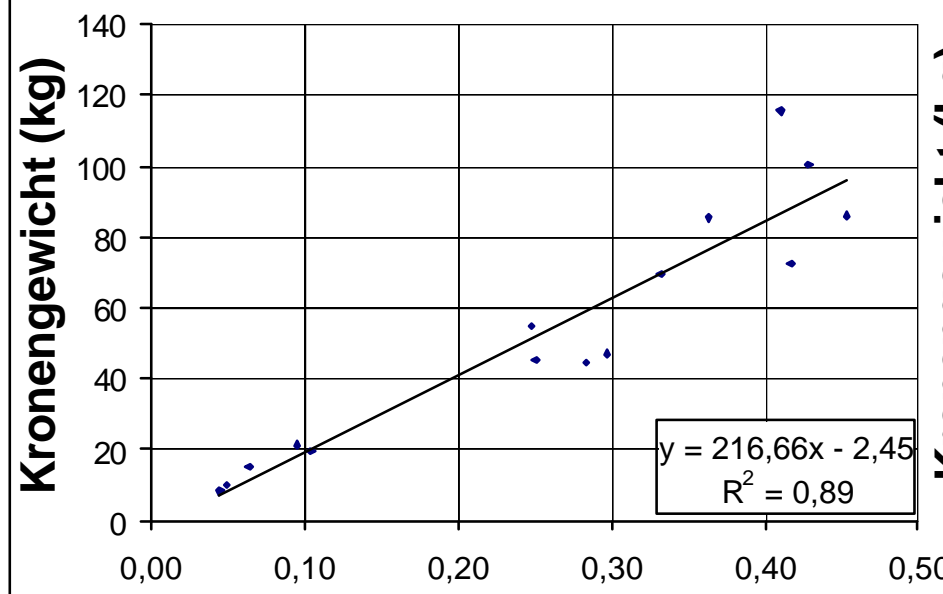

Stammvolumen $\left(\mathrm{m}^{3}\right)$

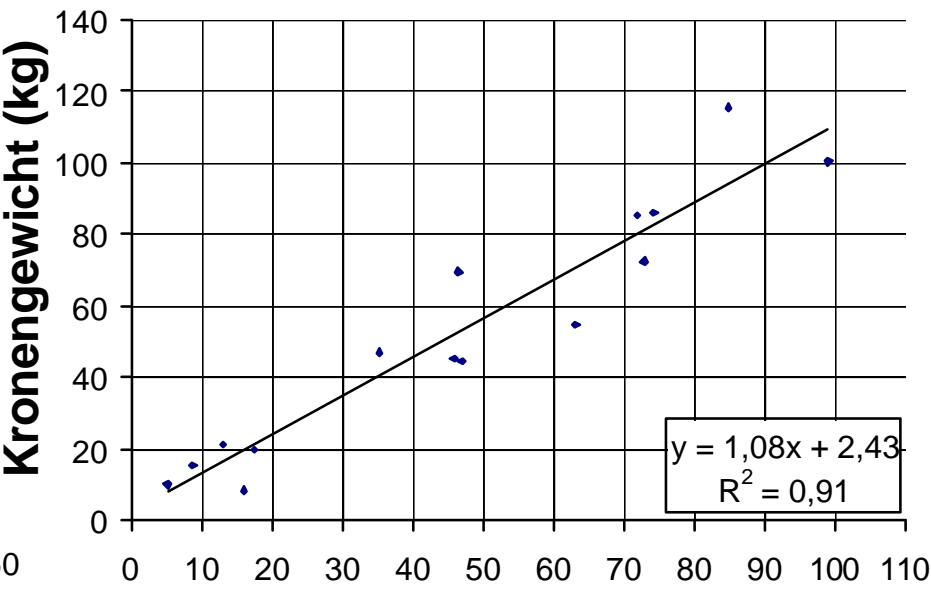

Wurzelvolumen $\left(\mathrm{dm}^{3}\right)$

Diagramm 5: Beziehungen zwischen Stamm-, Kronen- und Wurzelparametern; Krebeck, Fichte 41 jährig, Nadel / Blatt- und Kronengewicht (im Frischezustand). 


\section{Knesebeck, Fichte 20 jährig}
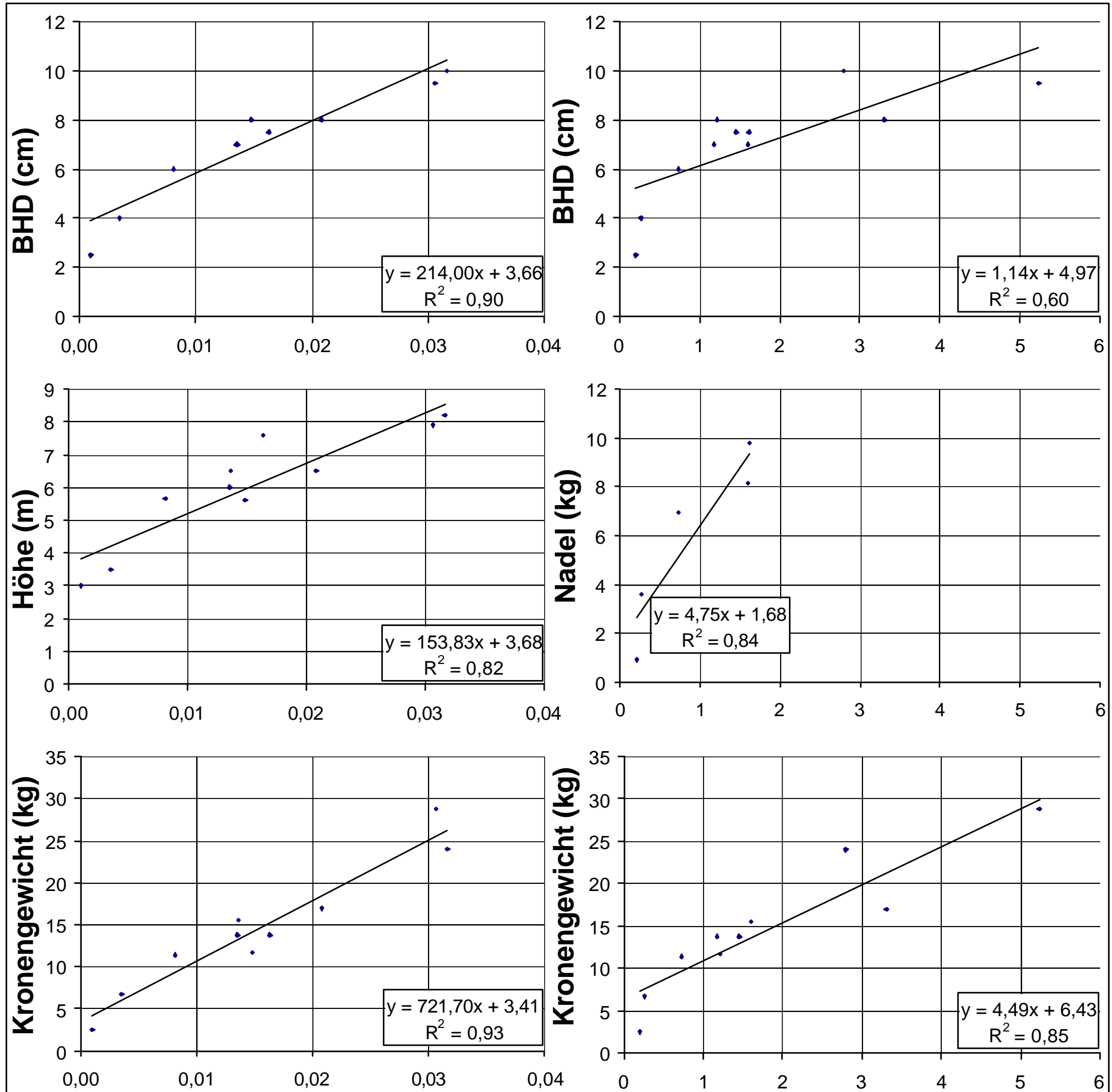

Stammvolumen $\left(\mathrm{m}^{3}\right)$

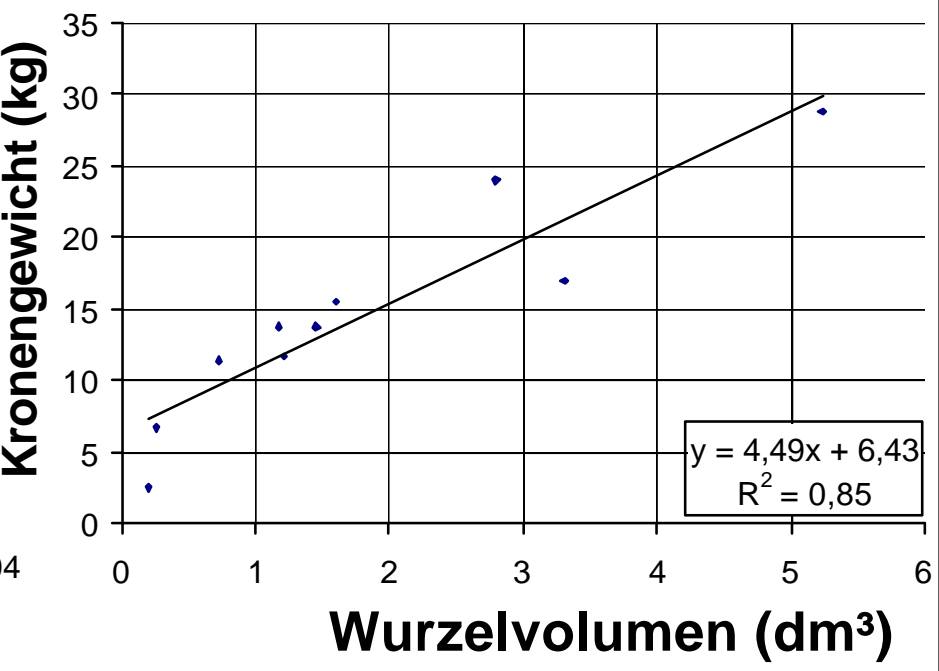

Diagramm 6: Beziehungen zwischen Stamm-, Kronen- und Wurzelparametern; Knesebeck, Fichte 20 jährig, Nadel / Blatt- und Kronengewicht (im Frischezustand). 


\section{Knesebeck, Fichte 31 jährig}
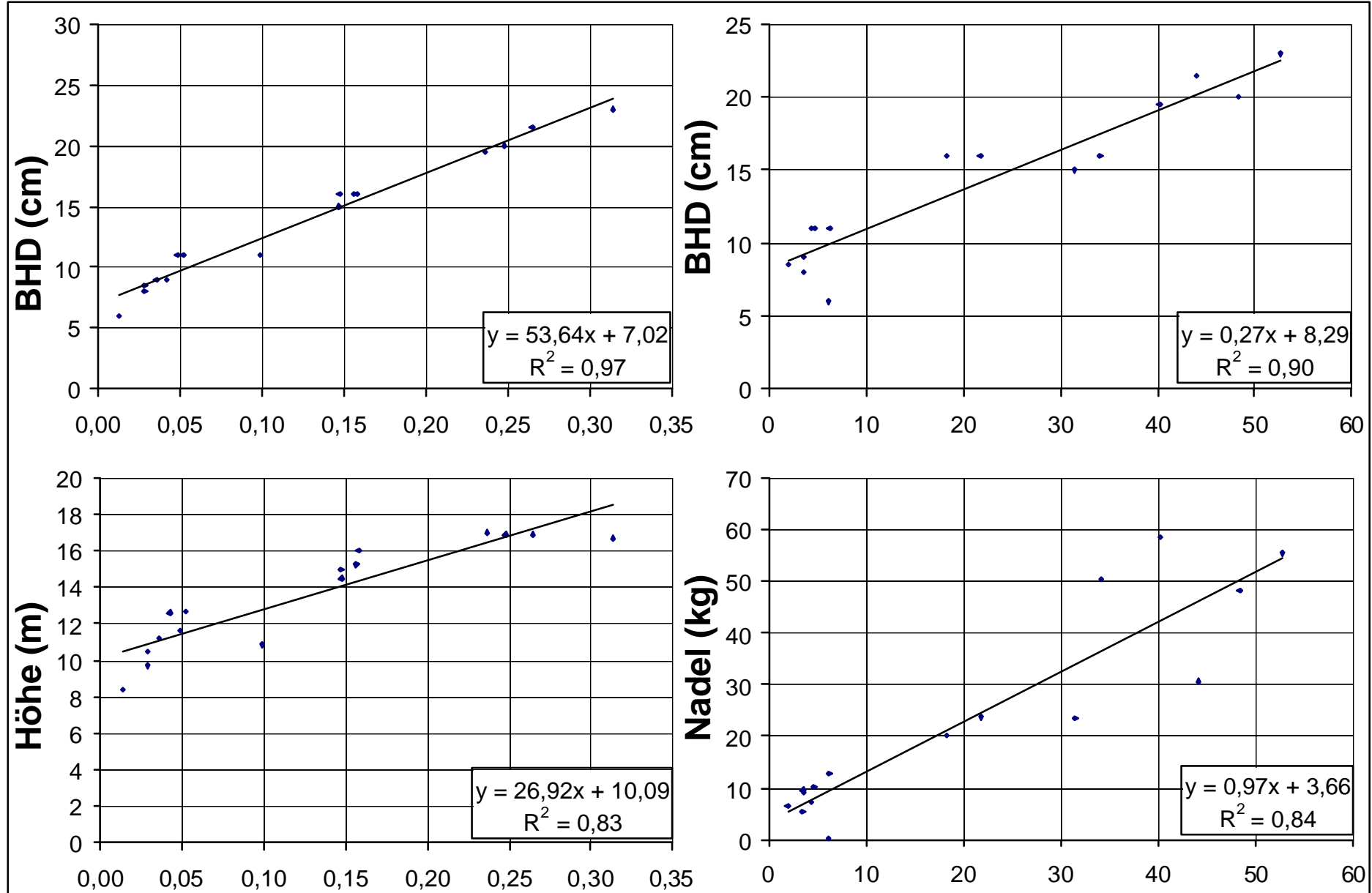

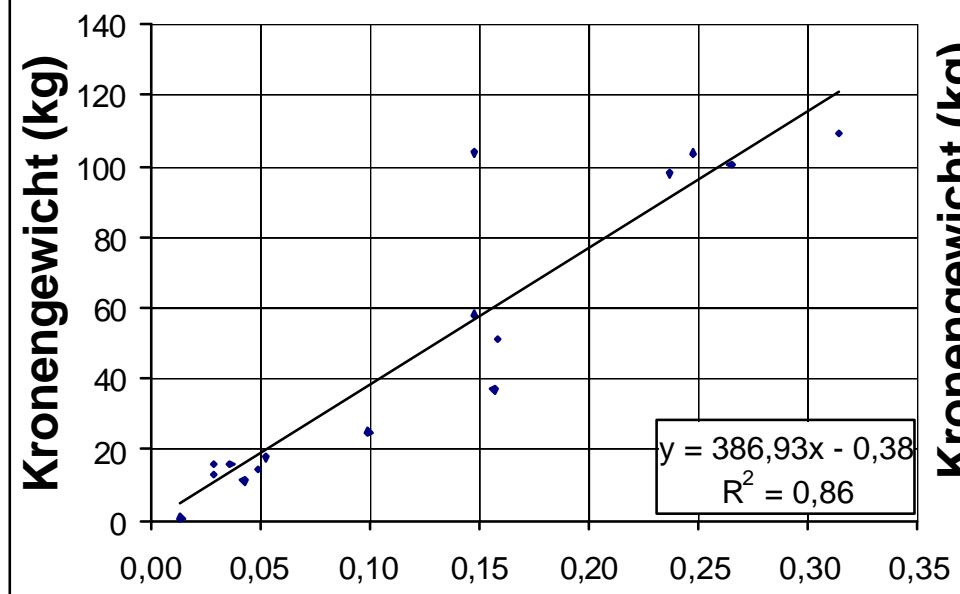

Stammvolumen $\left(\mathbf{m}^{3}\right)$

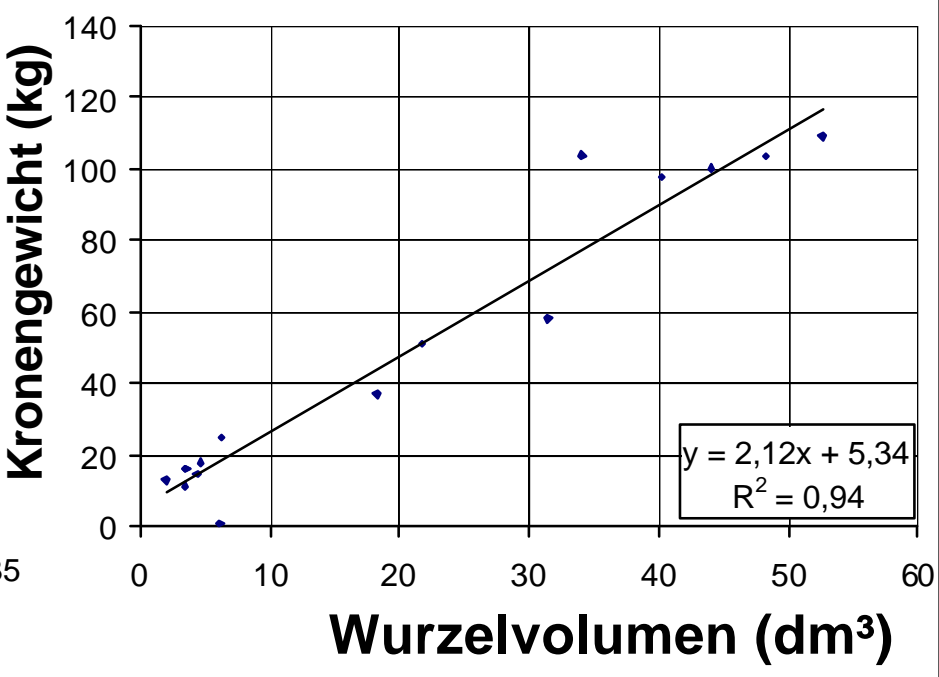

Diagramm 7: Beziehungen zwischen Stamm -, Kronen- und Wurzelparametern; Knesebeck, Fichte 31 jährig, Nadel / Blatt- und Kronengewicht (im Frischezustand). 


\section{Knesebeck, Fichte 50 jährig}
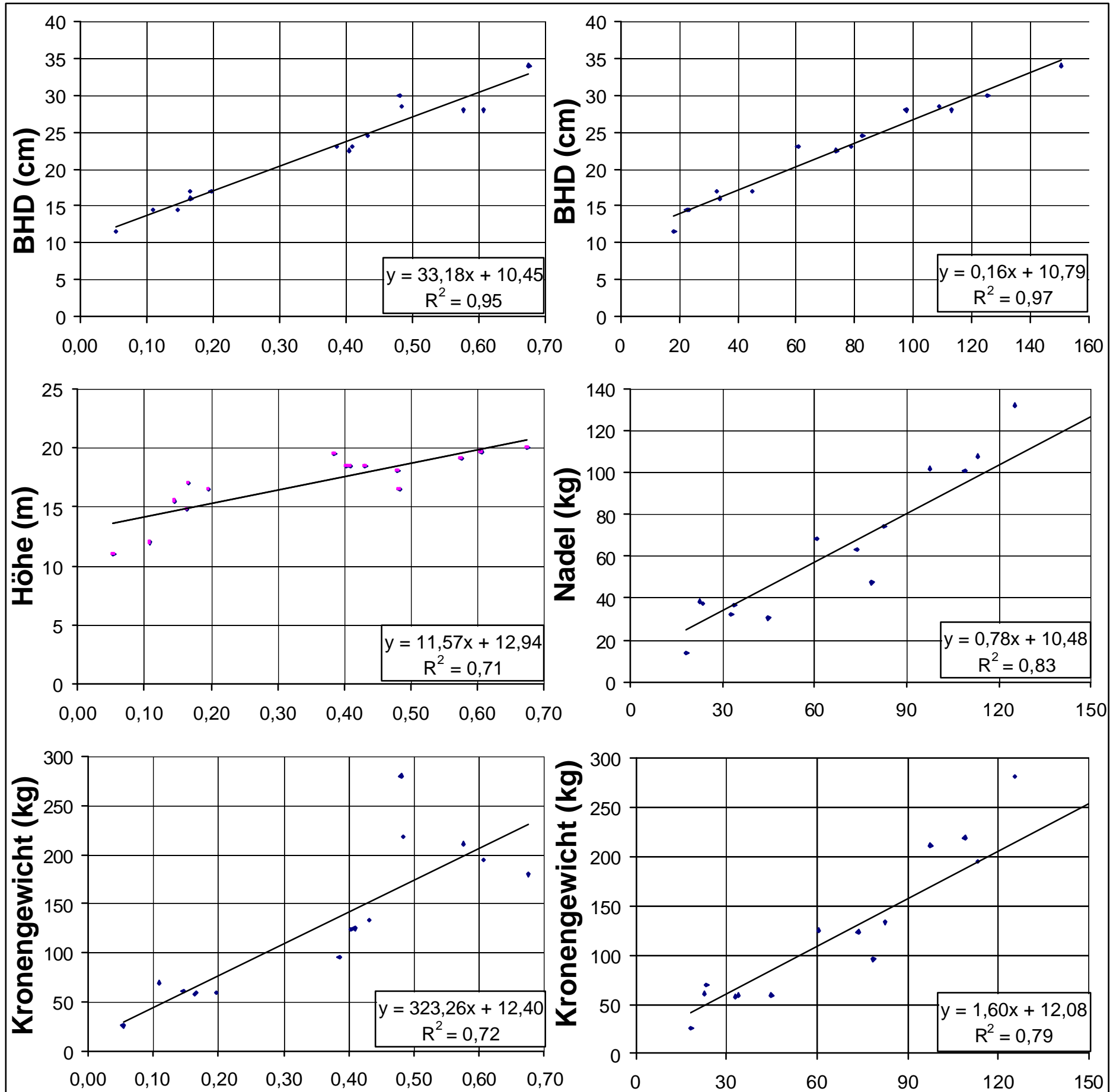

Stammvolumen $\left(\mathrm{m}^{3}\right)$

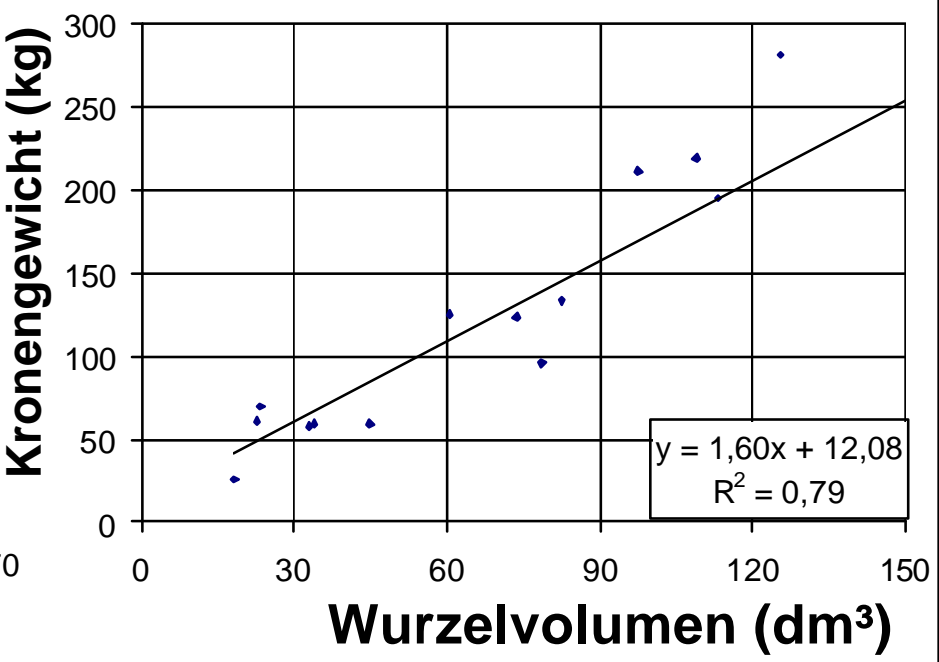

Diagramm 8: Beziehungen zwischen Stamm -, Kronen- und Wurzelparametern; Knesebeck, Fichte 50 jährig. Nadel / Blatt- und Kronengewicht (im Frischezustand). 


\section{Knesebeck, Kiefer 20 jährig}
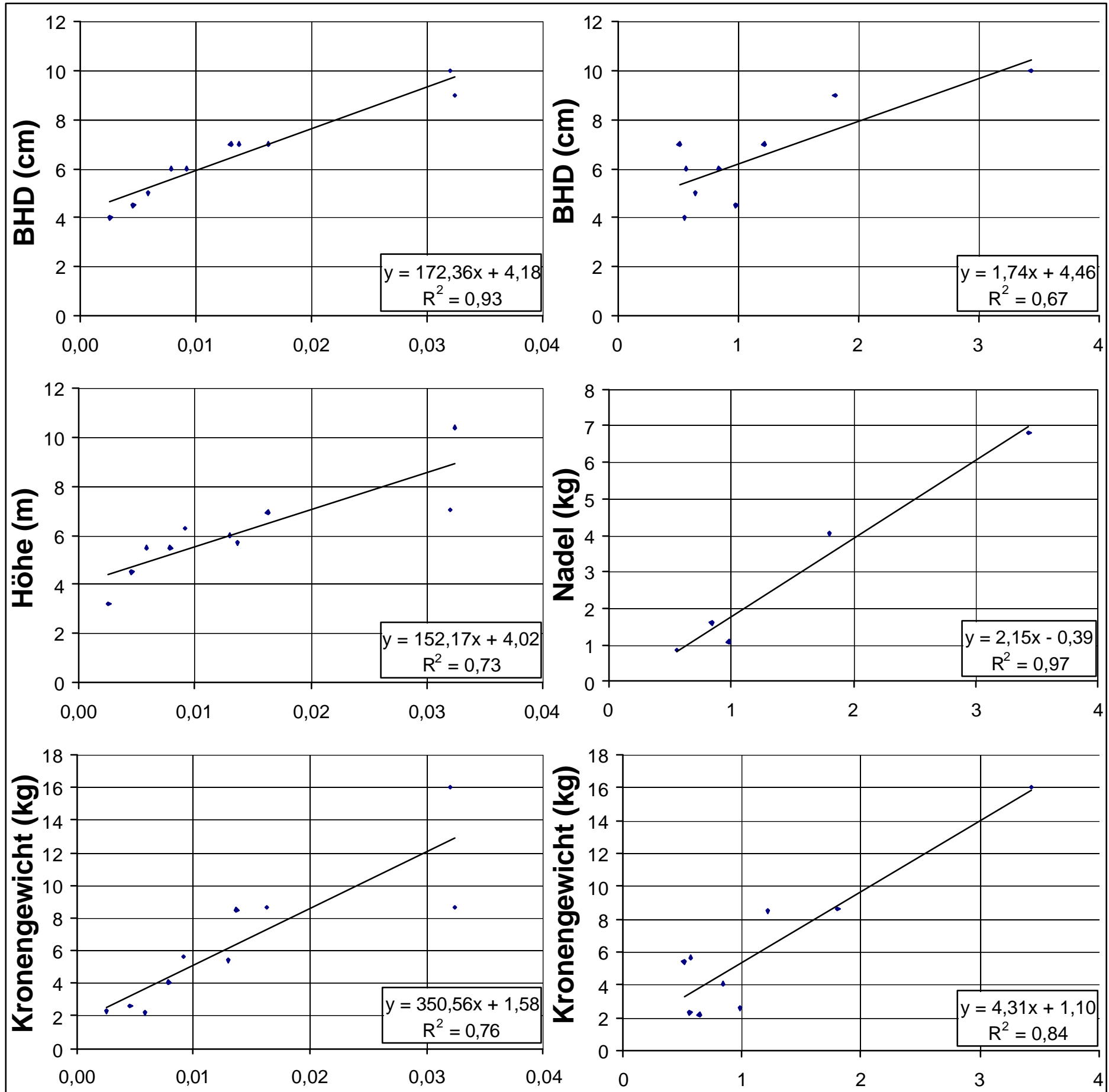

Stammvolumen $\left(\mathrm{m}^{3}\right)$

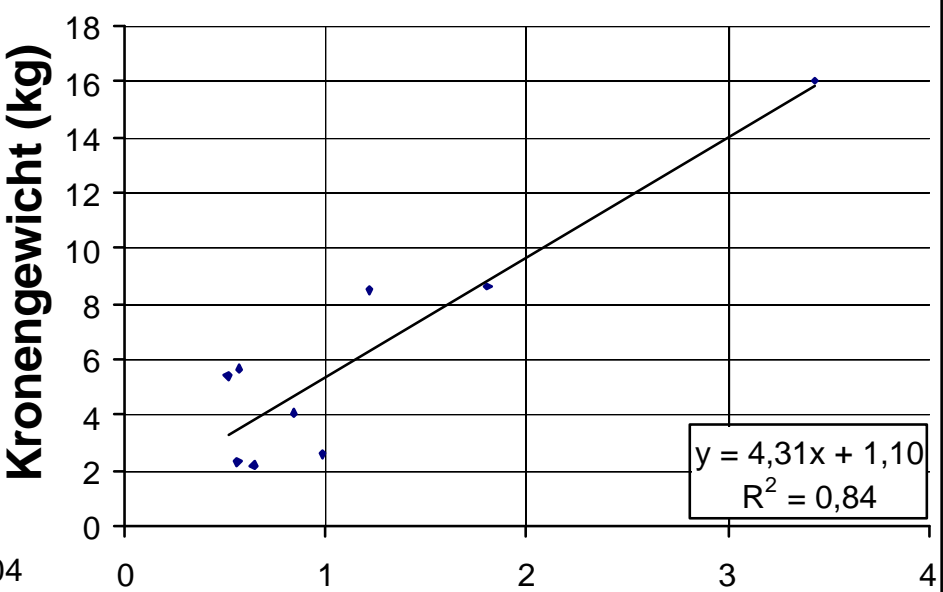

Wurzelvolumen $\left(\mathrm{dm}^{3}\right)$

Diagramm 9: Beziehungen zwischen Stamm -, Kronen- und Wurzelparametern; Knesebeck, Kiefer 20 jährig. Nadel / Blatt- und Kronengewicht (im Frischezustand). 


\section{Knesebeck, Kiefer 33 jährig}
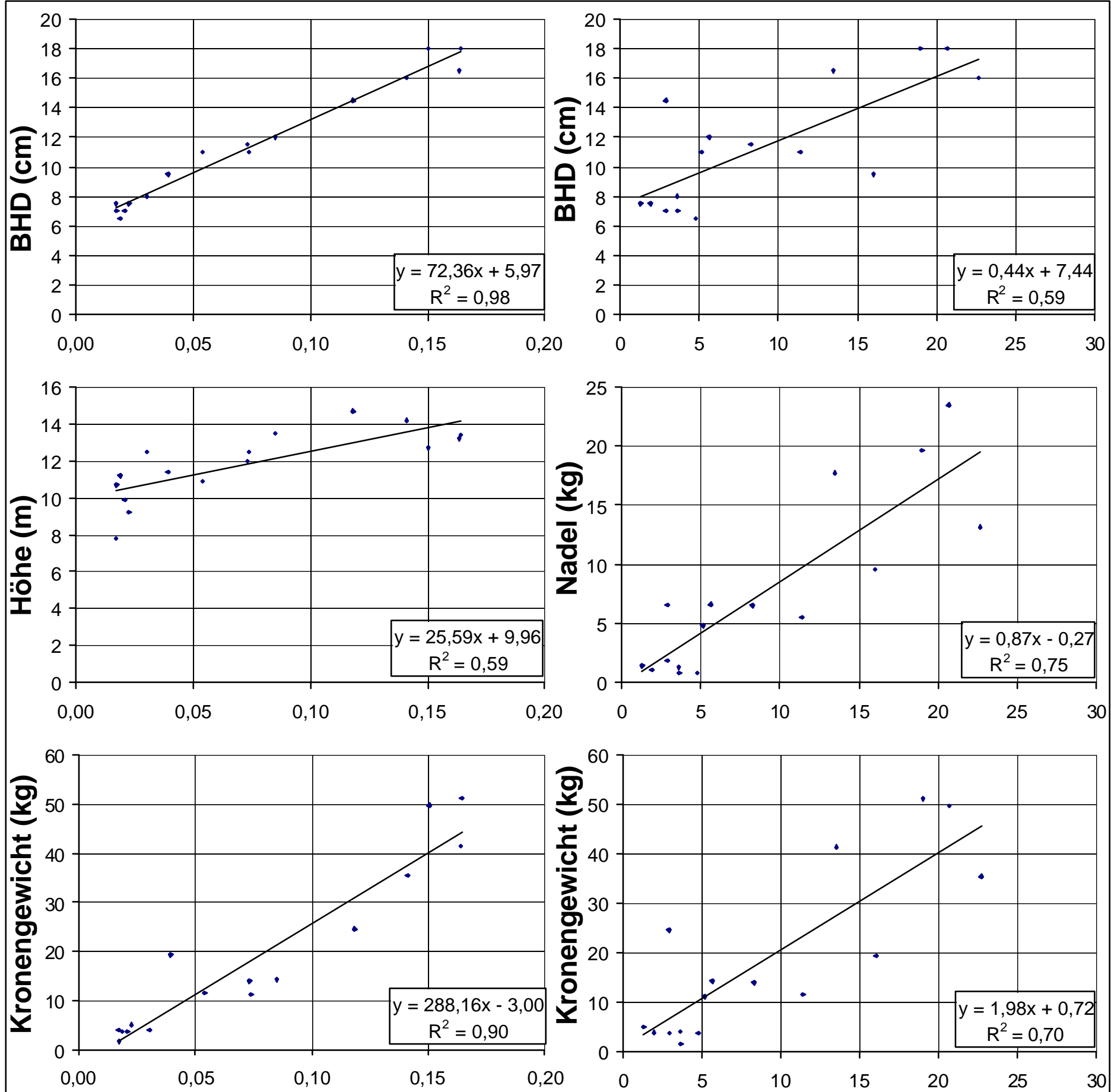

Stammvolumen $\left(\mathbf{m}^{3}\right)$

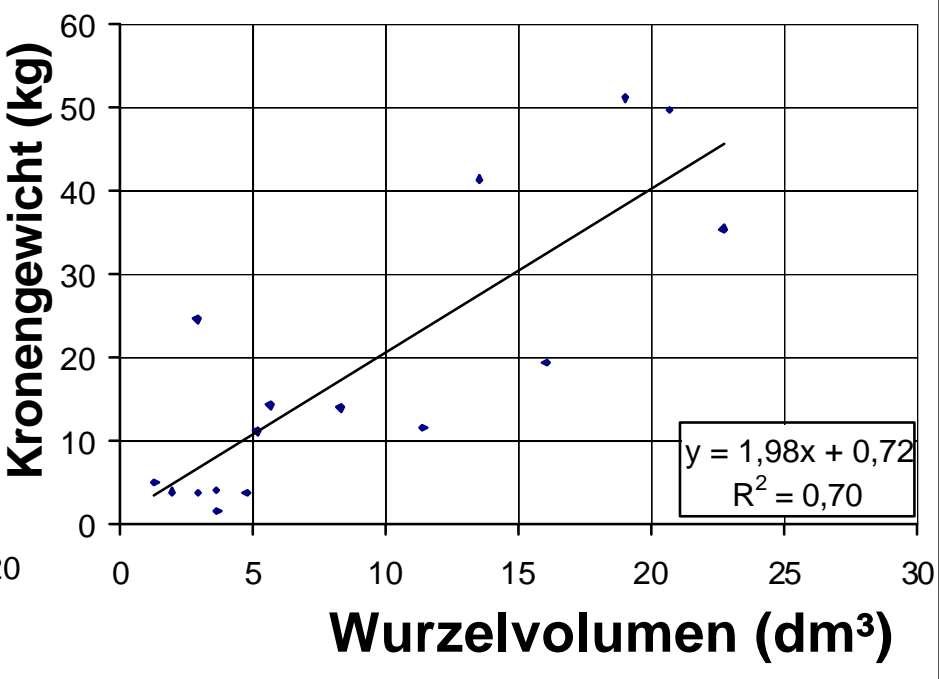

Diagramm 10: Beziehungen zwischen Stamm -, Kronen- und Wurzelparametern; Knesebeck, Kiefer 33 jährig, Nadel / Blatt- und Kronengewicht (im Frischezustand). 


\section{Holzminden, Eiche Pflanzung 22 jährig}
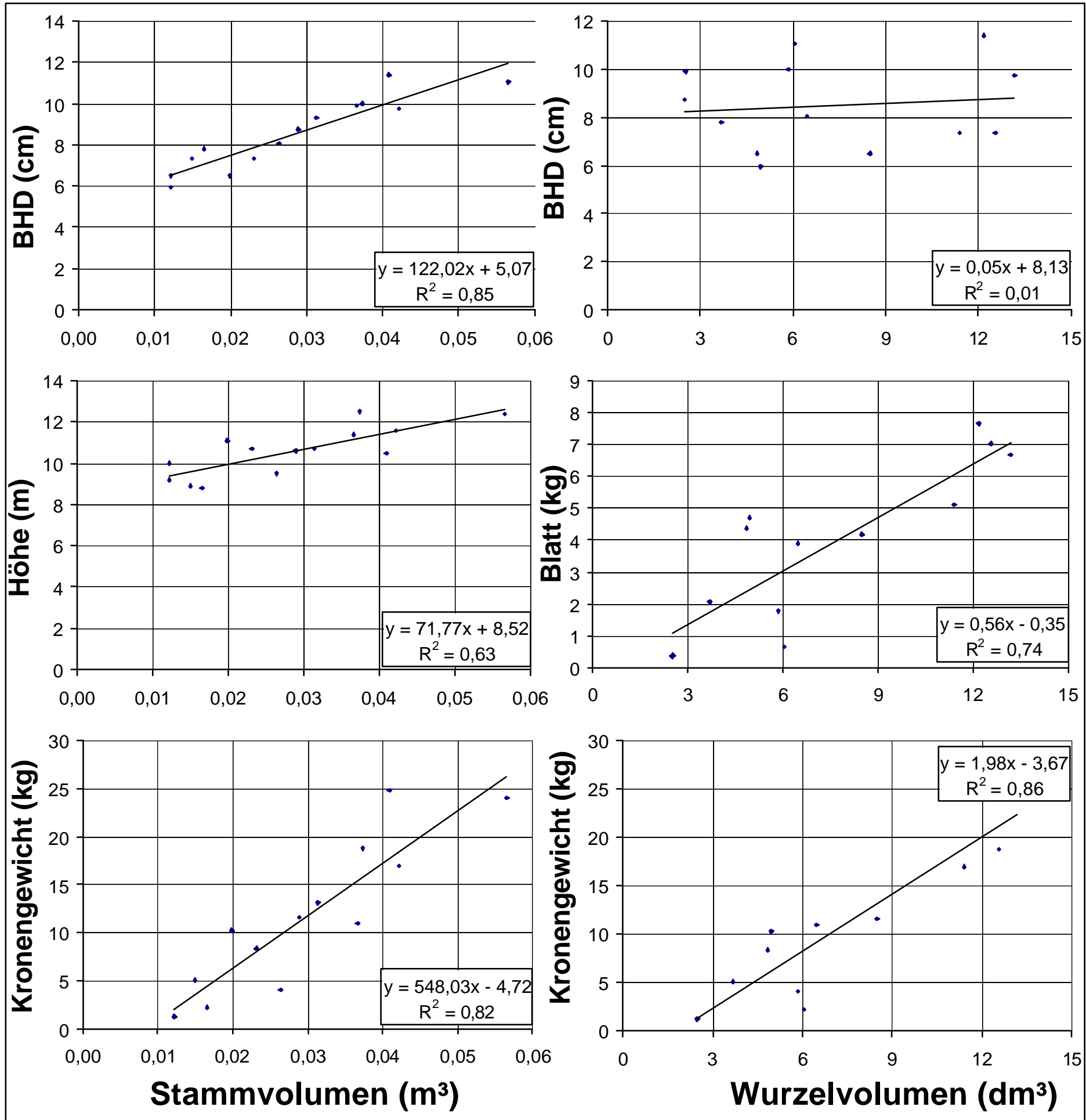

Diagramm 11: Beziehungen zwischen Stamm- Wurzel- und Kronenparametern; Holzminden, Eiche, Pflanzung 22 jährig, Nadel / Blatt- und Kronengewicht (im Frischezustand). 


\section{Holzminden, Eiche Saat 22 jährig}
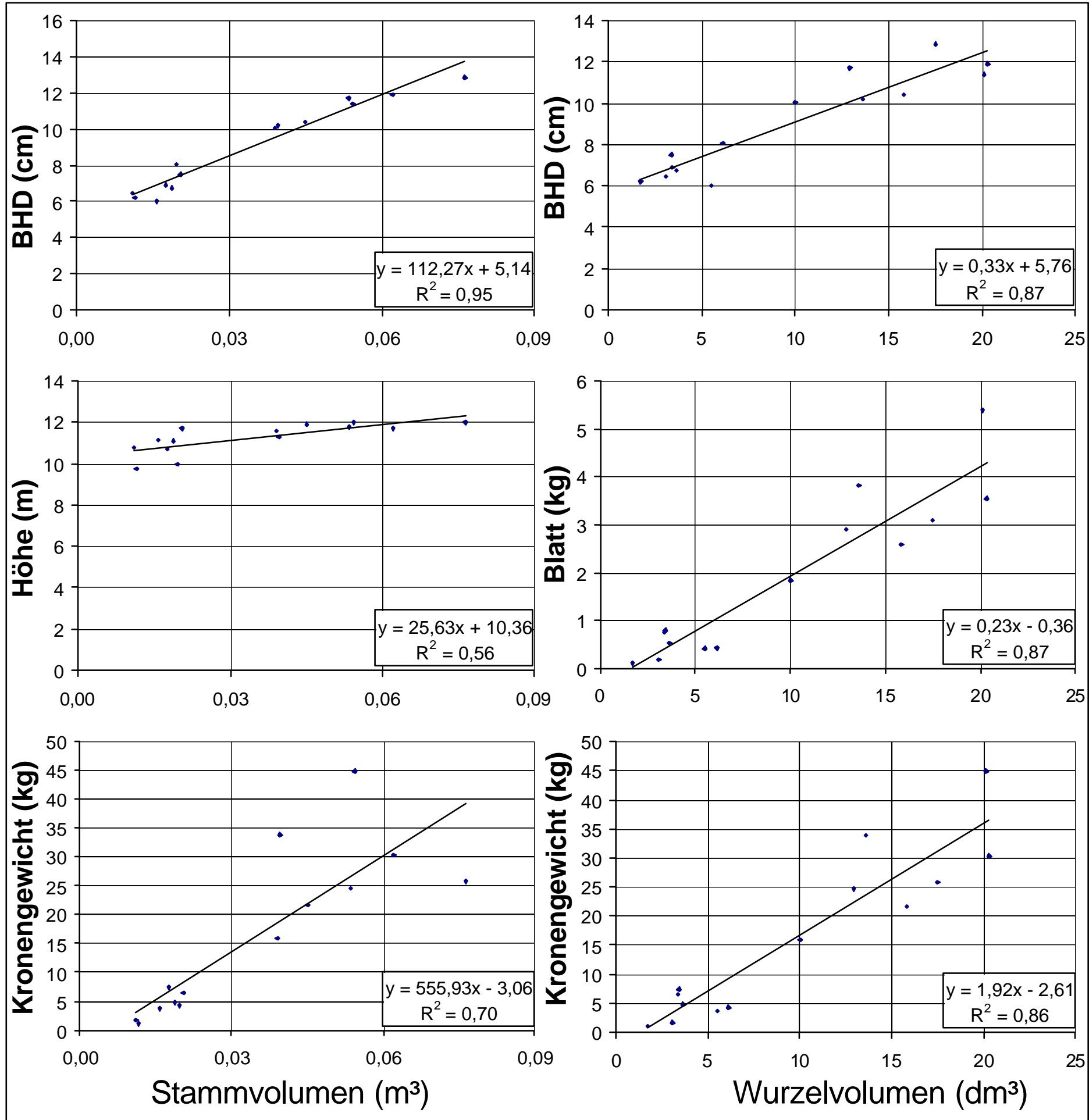

Diagramm 12: Beziehungen zwischen Stamm- Wurzel- und Kronenparametern; Holzminden, Eiche, Saat 22 jährig, Nadel / Blatt- und Kronengewicht (im Frischezustand). 


\section{Kattenbühl, Eiche Pflanzung 13 jährig}
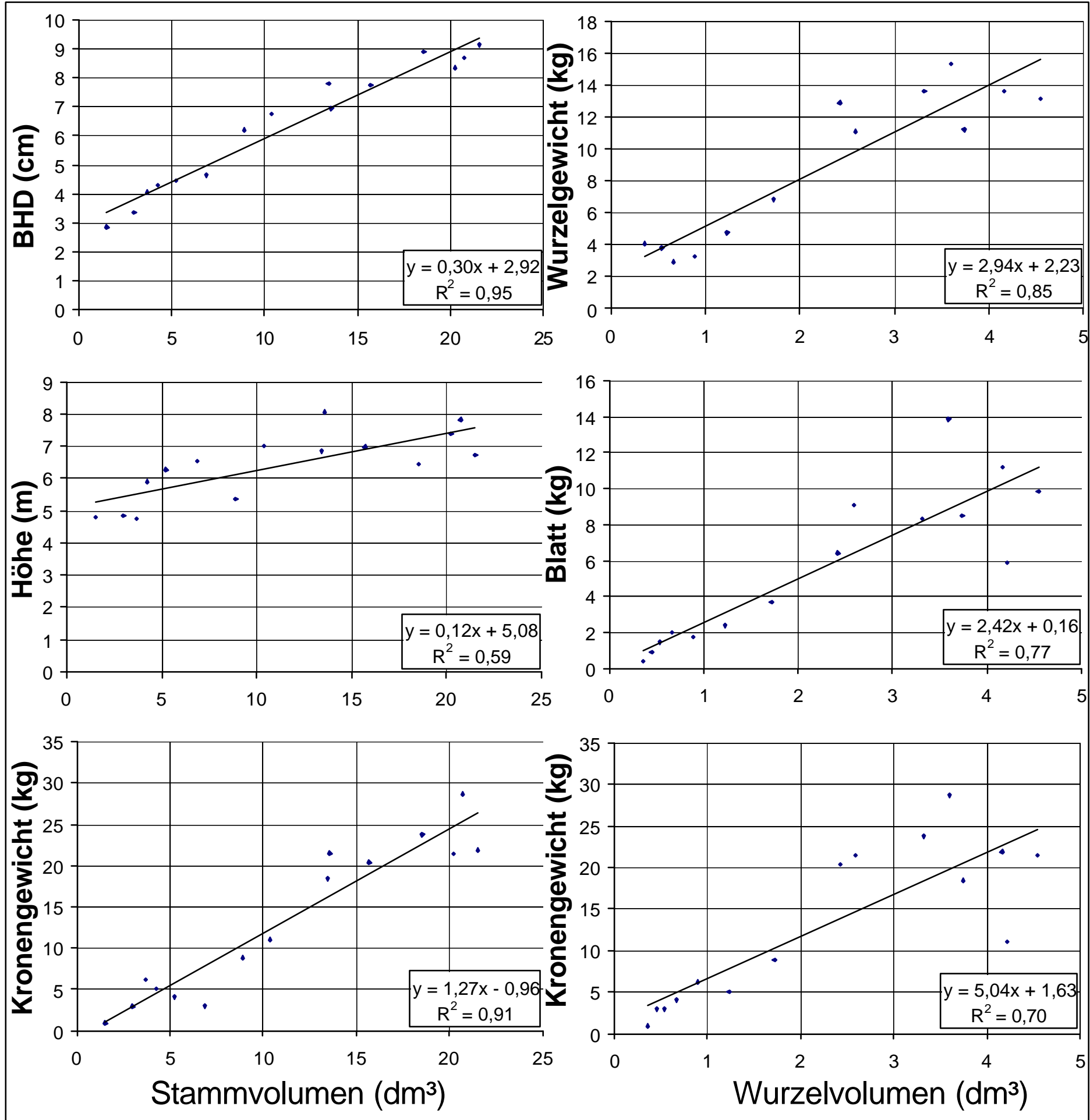

Diagramm 13 : Beziehungen zwischen Stamm- Wurzel- und Kronenparametern; Kattenbühl, Eiche, Pflanzung 13 jährig, Nadel / Blatt- und Kronengewicht (im Frischezustand). 


\section{Kattenbühl, Eiche Saat I 13 jährig}

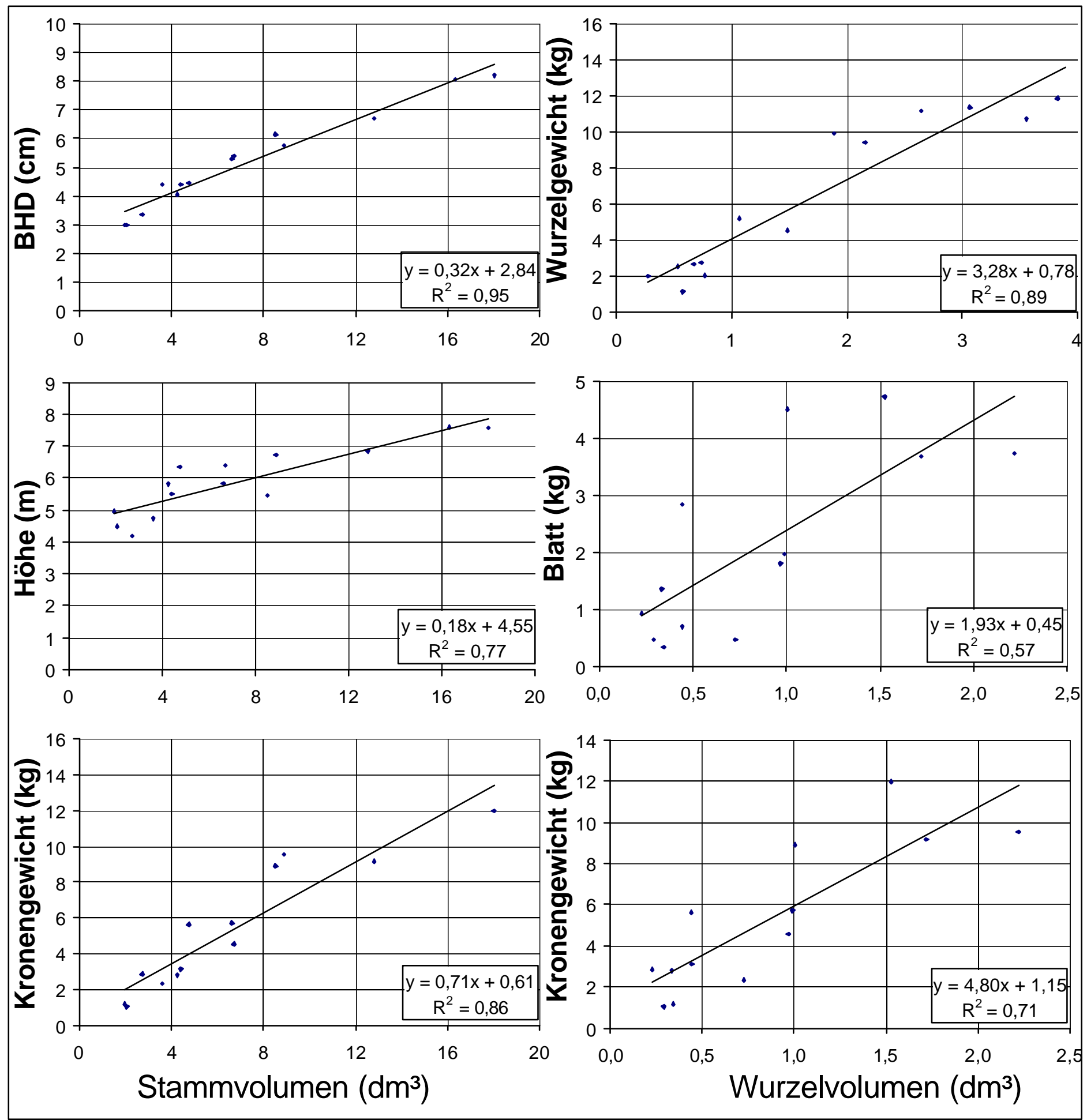

Diagramm 14: Beziehungen zwischen Stamm- Wurzel- und Kronenparametern; Kattenbühl, Eiche, Saat I 13 jährig, Nadel/ Blatt- und Kronengewicht (im Frischezustand). 


\section{Kattenbühl, Eiche Saat II 13 jährig}
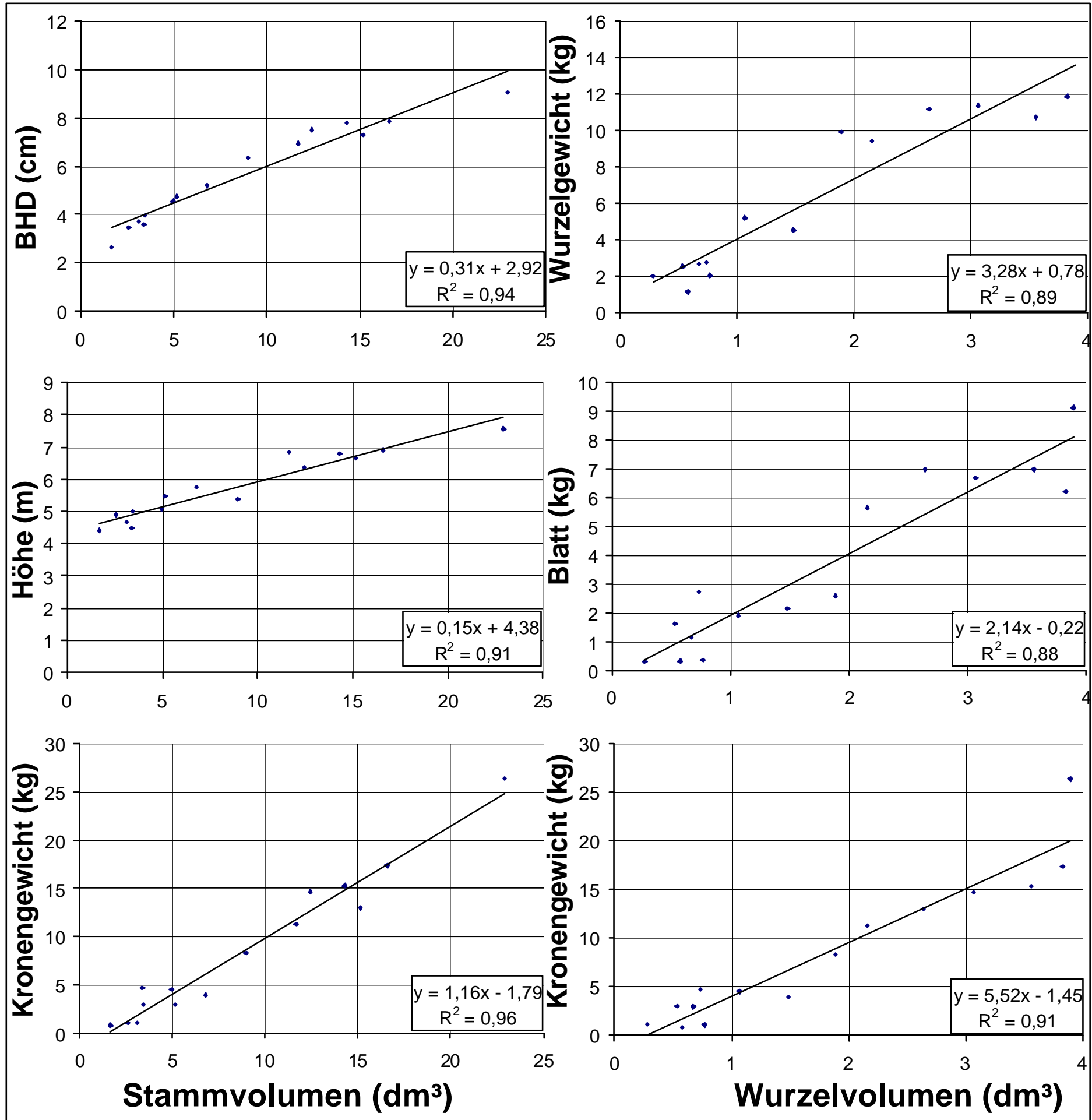

Diagramm 15: Beziehungen zwischen Stamm- Wurzel- und Kronenparametern; Kattenbühl, Eiche, Saat II 13 jährig, Nadel / Blatt- und Kronengewicht (im Frischezustand). 


\section{Maike Kuhr}

Persönliche

Angaben:

Ausbildung:
Familienstand: ledig

Staatsangehörigkeit: deutsch

Geburtstag: 26.02.1968

Geburtsort: Hildesheim

1974 - 1978 Grundschule Diekholzen

1978 - 1987 Gymnasium Andreanum

1987- 1989 Ausbildung zur landwirtschaftlich-technischen Assistentin, Braunschweig und Söllingen

1989 - 1990 Tätigkeiten im Institut für Geoanalytik, Technologiezentrum Hildesheim, und Arztpraxis für Kiefer-Gesichts-Mundchirurgie, Hildesheim

1990 - 1991 Forstpraktikum Forstamt Escherode

1991 - 1996 Forststudium an der Georg-August-Universität Göttingen mit Abschluß als Diplom Forstwirtin

- Diplomarbeit "fremdländische und seltene Baumarten im Stadtwald Hildesheim"

- Schwerpunkt Biologie/Ökologie

1996-1999 Wissenschaftliche Mitarbeiterin am Institut für Waldbau der Universität Göttingen 